

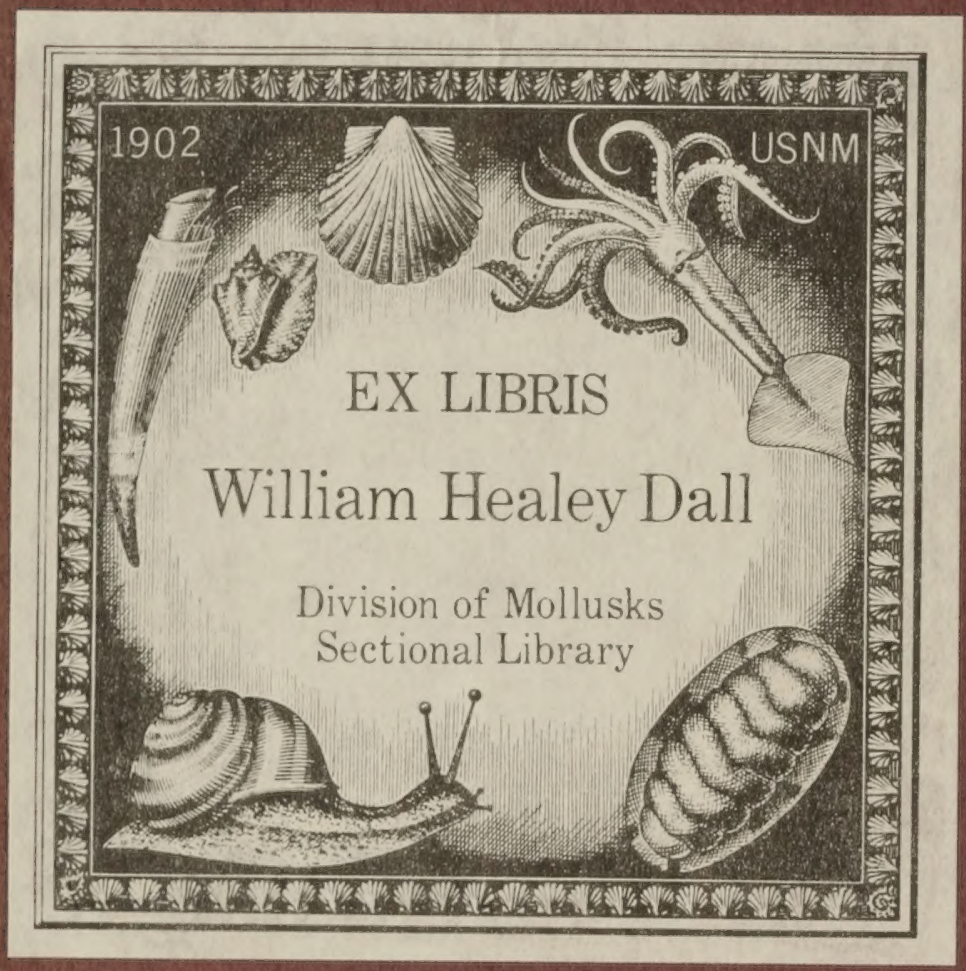


Q3.

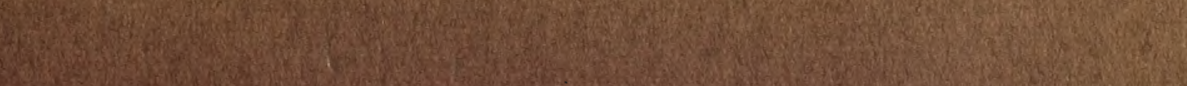

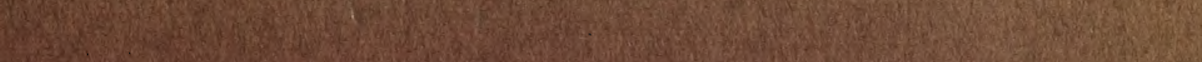
SW Pings: Sonity

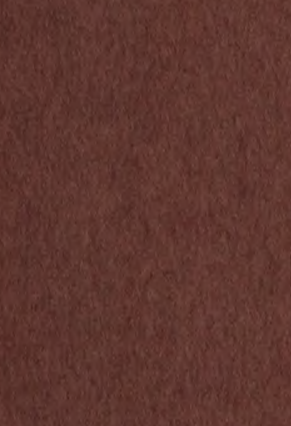
20.7. (2)

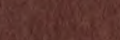
(1) 
$$
8
$$<smiles>C1C[AlH]1</smiles>

T5<smiles></smiles><smiles></smiles> 
K-2-a BOURG.

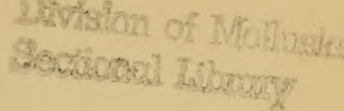





\section{A TÉEI A U X}

POBR SRRVIR A L'HISTOIRR DES

\section{MOLLUSQUES ACEPHALES}

DU SYSTEME EUROPEEN 


\section{5 \\ $A 1 B 77$ \\ 1880 \\ Moll. \\ M A T É R I A U X}

POUR SERVIR A L'HISTOIRE DES

\section{IIOLLUSOUES ACEEPIIALES}

DU

\section{SYSTÈME EUROPÉEN \\ PAR \\ J. R. Bon Bourguignat}

I

DE MAI I 880 A MAI I 88 I

\section{P O I S S Y}

IMPRIMERIE DE S. LEJAY \& C

16, rue des Dames, 16

$$
1880-1881
$$


"w:

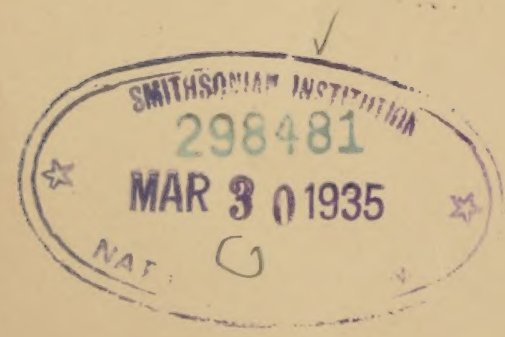


En 1877, dans ma Classification des familles et des genres des Mollusques terrestres, et fluviatiles du système europeen, j'ai mentionné quinze genres d'Acéphales, répartis en cinq familles, savoir :

\section{SPHARID E}

Corbicula, Muhlfoldt, 1811.

Sphærium, Scopoli, $177 \%$.

Pisidium, C. Pfeiffer, 1821.

Eupera, Bourguignat, 1854 .

\section{UNIONIDAE}

Unio, Philippsson, 1788.

MAI. 1880. 
Margaritana, Srhumacher, 1817.

Leguminaia, Conral, 186\%.

Pseudodon, Gould, 1844.

Anodonta, Cuvier, 1798.

\section{IRIDINID $A$}

Mutela, Scopoli, 1777.

Jolya, Bourguignat, 1877.

Spatha, Lea, 1838.

Pliodon, Conral, 1833.

\section{RTHERID $A$}

Etheria, Lamarck, 1807.

\section{DREISSENIDA}

Dreissena, Vun Beneden, 1833.

Or, depuis cette époque, gràce à de nouvelles études, grâce surtout aux magnifiques découvertes de mes amis, j'admets six familles et dix-neuf genres.

Voici cette nouvelle classification :

\section{SPHARIDA.}

Corbicula, Muhlfeldt, 1811 (Cyrena (pars) Lamarck, 1806. - Cyanocyclas, Ferussac, 1818).

Sprtereux, Scopoli, 1777, et Bourguignat, 1833 et 18304 (Nux, IHumphrey, 1796. - Cyclas, Bruguières, 
1791. - Cornea et Pisum, Wullfeldt, 1811. - Corneocyclas, Ferussac, 1818. - Calyculina, Clessin, 1872 (pour les sphéries calyculées).

Pisidum, C. Pfeiffer, 1821, et Jenyns, 1832. -

(Pera, Euglesa et Cordula, Leach, mss, 1819. Pisum, Gray, 183̋3.)

Eupera, Bourguignat, 185\%. (Limosina, Clessin, 1874.)

\section{UNIONID $E$}

Pitaraonia, Bourguignat, 1878, in Sched.

Unı, Philippsson, 1788 (non Retzius).

Margartana, Schumacher, 1817.

Leguminais, Conrad, 186å. (Microcondylæa, Vest, 1867, et Clessin, 1874.)

J'avais, dans ma première classification, divisé les espèces de ce genre en Leguminaia proprement dites pour les formes asiatiques, et Uniopsis (Swainson) pour celles italiennes. Je reconnais actuellement que les espèces italiennes doivent êtrè réunies aux vraies Leguminaia dont elles possèdent tous les signes distinctifs, et qu'elles n'ont pas le moindre rapport arec les Uniopsis de Swainson. Les principales espèces de ce genre sont: les Leguminaia Mardiniensis, Saulcyi, Michoni, Tripolitana de Syrie et les 
Bonellii, curvata (Rossmüssler, fig. 746,1842 ) de Lombardie.

Pseudodon (pars), Gould, 1844.

J'avais divisé ce genre en Movodontiva (Conrad, 1852, - Alasmodonta, Ferussac, 1820, et Bourguignat, 1870 (non Alasmodonta de Say, 1818),-Monocondyliea (pars), Lea, 1839 et 1864 (non Monocondylæa de d'Orbigny), pour les espèces asiatiques comme les Pseudodon Opperti, rhomboideus, piestius, Churchillianus, Babylonicus, pachyolenus, etc., et, en Pseudanodonta pour les formes européennes, comme les Berlani, Penchinati, Kilettii, etc. Mais j’ai reconnu depuis que toute la série européenne méritait d'être classée dans un genre spécial, celui de :

Pseudanodonta, Bourguignal (c. sous-génér.), 1877. Anodonta, Cuvier, 1798 (Anodontites, Bruguières,

1791. - Anodon, Oken, 1815).

Colcetoptenum, Bourguignat, 1880.

\section{IRIDINID $A$}

Mutela, Scopoli, 1777. (Mutel, Adanson, 1757.Iridina, Lamarck, 1819.)

Cirandandi, Bourguignat, 1878, in sched.

Spatua, Lea, 1838.

Pliodon (pleiodon), Conrad, 1835. 


\section{JOLYD E}

JoLYA, Bourguignat, 1877.

J'avais classé ce genre dans la famille des Iridini. $d a$, mais de nouvelles études m'ont porté à le séparer et à le metire dans une famille spéciale.

\section{ATHERID E}

Átheria (E'theria), Lamarck, 1807.

\section{DREISSENID $E$}

Dreissena, Van Beneden, 183 .

Tel est l'ensemble des familles et des genres des Mollusques Acéphales du système européen.

Je dois dire un mot, je crois, sur la nouvelle méthode de mensuration, d'après laquelle je décris (sauf les Ethéries dont les contours ne sont jamais constants) toutes les espèces dont je vais domner les caractères.

Jusqu'à présent, on ne trouve, chez les auteurs, que trois mesures : celles de la longueur, de la hauteur (ou largeur) et celle de l'épaisseur. Or, ces mesures sont tout à fait insuffisantes, attendu qu'avec elles il est impossible de reconnaître le point exact 
de la longueur, de la hauleur et encore moins de l'épaisseur.

Chez les Acéphales, en effet (je prends les Anodontes, par exemple), suivant les espèces, l'épaisseur peut être plus ou moins rapprochée des sornmets ou reculée vers les parties inférieure ou postérieure; la hauteur peut être plus ou moins éloignée du bord antérieur, selon que ce bord prend plus de développement, et ainsi de même pour la longueur, qui ellemême dépend de la forme des deux contours antérieur et postérieur.

Aussi, ces trois mesures sont-elles si défectueuses que je mets au défi le naturaliste le plus habile de reconstruire sur le papier les contours d'une Anodonte.

J'ai donc cru de mon devoir de modifier du tout au tout le mode de mensuration.

’ar la méthode que je propose, le conchyliologue le moins exercé pourra, sans gravures, reproduire la forme d'un Acéphale, et arriver, par elle, à la connaissance de l'espèce.

Un mot auparavant sur la manière de placer un Acéphale.

La partic postérieure est celle où se trouve le plus fort ligament, le plus souvent externe. C'est ordinairement la partie la plus développée, sauf chez les 
Pisidies, les Sphæries et les Corbicules, où elle est plus courte, ou parfois égale, ou enfin un peu plus forte que la partie antérieure.

La partie antérieure est nécessairement l'opposé de la postérieure.

Je place l'Acéphale debout sur son bord palléal, les sommets en dessus, la partie postérieure de mon côté et l'antérieure en face.

Dans cette position, la valve dextre est celle qui correspond à ma droite, la sénestre à ma gauche.

Pour prendre la mensuration, je renverse la ccquille sur le côté, de manière à avoir la partie antérieure à ma gauche, la postérieure à ma droite, de façon à ce que les sommets soient culminants.

J'abaisse alors une perpendiculaire juste dans mon rayon visuel, perpendiculaire qui, des sommets, tombe sur un point quelconque du bord palléal. Or, toute la région à gauche de cette ligne devient pour moi la partie antérieure, toute celle de droite, la pos. térieure.

Je prends sur cette perpendiculaire le point de la plus grande distance du bord antérieur, et, de ce même point, celui du rostre postérieur; je tire ensuite une ligne de ce rostre aux sommets, ce qui me donne, au moyen de ces mesures, quatre points fixes.

Ces quatre points, celui des sommets avec ceux de la base de la perpendiculaire, du côté antérieur et de 
l'extrémité postérieure, sont les points fondamentaux de la forme d'une espèce. Une fois qu'on les connaît, qu'on les a marqués sur le papier, il est facile, au moyen des lignes secondaires, d'arriver au tracé exact des contours.

Ainsi, pour obtenir la direction de la région dorsale, je relie par une ligne la base de la perpendiculaire à l'angle postéro-dorsal, et, en réunissant ce point à celui des sommets d'un côté et à celui du rostre de l'autre, je possède alors le contour à peu près exact.

Mais pour l'avoir avec exactitude, dans le cas où la hauteur maximum ne serait pas celle de la perpendiculaire, ce qui arrive rarement chez les Anodontes, sauf chez les Unios, les Muteles, etc. je prends alors la plus grande hauteur et jubtiens par elle la mesure des modifications.

Etc., etc.

Quant au maximum d'épaisseur des valves, comme il peut se trouver (suivant les espèces) ou à la partie antérieure, ou plus ou moins rapproché des sommets ou du bord postérieur, il est nécessaire de noter sa distance arec celle des quatre points principaux de la mensuration.

La planche que je donne ci-jointe, sur laquelle j'ai fait reproduire quelques modeles de mesurage, fera 


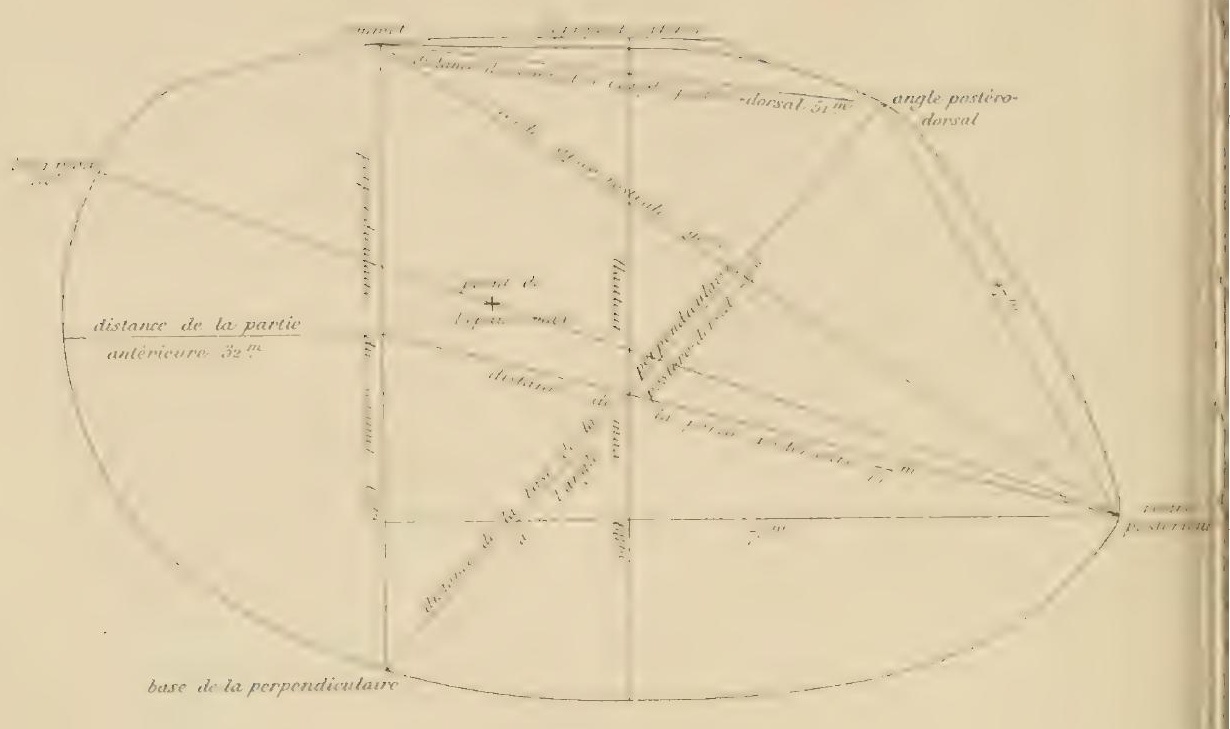

P'sendanodonta procelara.

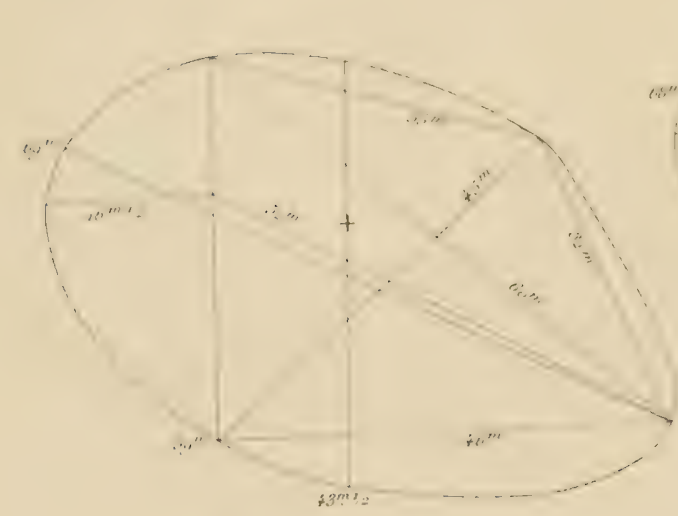

P'seudanodonta Bertani.

Lith. Becquel, Impr

MEXSURATIOX

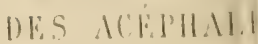




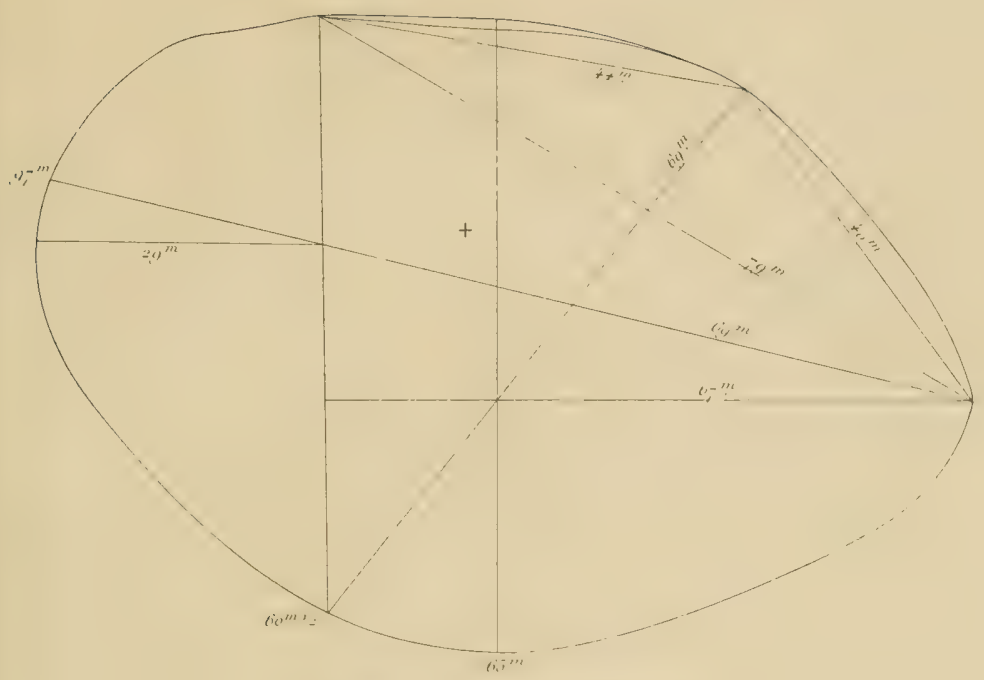

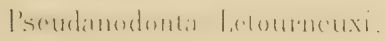

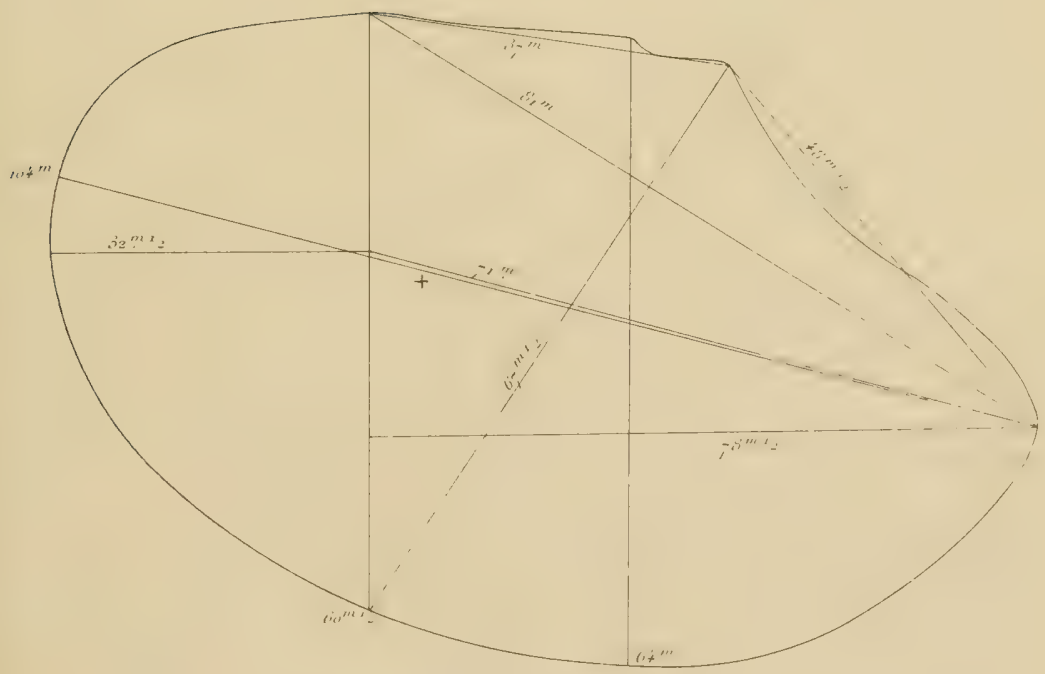

Anodonta resima. 

mieux comprendre que toutes les explications possibles cette nouvelle méthode.

Je sais bien qu'en proposant cette méthode, dont l'avantage est incontestable, ainsi que le reconnaîtront les vrais savants, je sais bien, dis-je, que je vais contrarier les vieilles habitudes, dérouter l'ancienne. école et attirer sur moi les colères et la malveillance de toute la bande. Mais c'est là le moindre de mos soucis. 



\section{NOTICE MONOGRAPHIQUE}

SUR LES

\section{PSEUDANODONTES}

\section{Pseudanodonta}

Pseudanodonta, Bourguignat, in Sched. 1876, et Classif. fam. gen. mull. ter. fluv., système Européen, p. 55, 1877.

Les espèces de ce genre sont des coquilles très inéquilatérales, à valves comprimées, aplaties, de forme subrhomboïde, subtétragone, ovalaire, ou bien allongée ou subtriangulaire, recouvertes d'un épiderme brillamment coloré (sauf sur les contours, où il est ordinairement feuilleté), et enduites à l'intérieur d'une superbe nacre blanche, bleue, rougeâtre ou irisée de toutes nuances.

Valves toujours bâillantes : $1^{0}$ à la partie inféro-antérieure; $2^{\circ}$ à la partie comprise entre l'angle postéro-dorsal 
et le rostre postérieur. La région inféro-antérieure est la plus bâillante. Quant à celle postéro-dorsale, elle est parfois (suivant les espèces) presque nulle ou souvent réduite à une simple fente.

Sommets aplatis, comprimés, à pointe aiguë.

Deux ligaments, comme, du reste, dans toule la famille des Unionide

$1^{\circ}$ Un antéro-interne, feuilleté (sans cortex), tantôt filiforme, tantôt occupant toute l'épaisseur de la charnière, commençant au niveau antérieur de l'impression cardinale et se poursuivant en arrière des sommets sousl'autre ligament, où il s'épanouit souvent très loin sur la lame latérale, sous la forme d'une lunule parfois profonde et très développée, où vient s'étaler l'extrémité de la tunique extérieure de ce ligament.

$2^{\circ}$ Un postéro-externe (symphynoté dans le jeune âge, c'est-à-dire recouvert par une membrane calcaire), commençant à la pointe des sommets pour se poursuivre presque jusqu'à l'angle postéro-dorsal, où il se termine en dedans par une lunule plus ou moins grande.

Charnière des plus simples, composée, cependant, de deux parties:

$1^{\circ}$ La partie cardinale qui comprend toute la région qui s'étend d'une extrémité à l'autre du ligament antéro-interne. Cette cardinale, pourvue tantôt d'une lame filiforme saillante plus ou moins longue, tantôt de une ou deux lames tuberculiformes, allongées, triangulaires ou subtétragones, suit le contour du ligament antéro-interne.

20 La partic latérale, dont la portion antérieure est toujours envahie par le ligament antéro-interne, s'étend de la pointe des sommets, sous la forme d'un renflement lamelliforme plan jusqu'à la lunule du ligament postéro-ex- 
lerne. Cette partie est toujours unie, plane et horizontale.

En 1876, j'avais regardé les Pseudanodontes comme un sous-genre des Pseudodon, mais maintenant je crỏis qu'il y a lieu de séparer les espèces de ces deux coupes génériques. Les Pseudodon, en effet, possèdent toujours à la région cardinale deux énormes tubercules triangulaires très saillants qui s'emboittent l'un près de l'autre : celui de la valve droite toujours en avant de celui de la valve sénestre.

Je pense également que les Pseudanodontes ne peuvent être assimilées aux vraies Anodontes, attendu que celles-ci possèdent une charnière, sans denticulation, réduite à la plus simple expression, une charnière représentée seulement par une ligne à peine ondulée, très mince, ininterrompue d'une extrémité à l'autre.

Considérées d'après leurs formes extérieures, les Pseudanodontes forment une coupe générique intermédiaire entre les Pseudodon et les Anodonta, coupe plus rapprochée, à mon avis, des Pseudodons que des Anodontes.

Les espèces de ce nouveau genre sont toutes de l'Europe où elles ont été constatées dans les régions du bassin danubien, dans l'Allemagne du Nord, ainsi qu'en Russie, en Norvège, en France, en Angleterre, etc. Je n'en connais point des régions méditerranéennes, comme des presqu'îles hispanique, hellénique et italique, bien qu'une ou deux formes aient été signalées en Lombardie.

Parmi les Pseudanodonta, sept d'entre elles ont déjà été publiées soit sous le nom générique d'Alasmodonte (Penchinati, Berlani, 1870), soit sous celui d'Anodonta (complanata, Klettii, 1830̃. - elongata, 1836, - Grate- 
loupiana, Normandi, 1849). Sur ces sept espèces, six nnt été bien définies, mais une, la première créée, la complanata, a été comprise d'une façon différente par chaque auteur qui a eu l'occasion d'en parler. Sur les cinquantesix malacologistes qui, à ma connaissance, ont décrit plus ou moins exactement ou qui ont simplement mentionné cette espèce, huit seulement en ont donné la représentation. Or, les huit figures et les huit descriptions de ces auteurs sont toutes dissemblables.

La cause première de ces dissemblances est due à l'erreur de Rossmässler, le créateur de l'espèce qui, après avoir décrit et fait représenter (fig. 68), en avril 183ð̃, la complanata, s'est avisé, l'année suivante, de reproduire (fig. 283) une autre forme sous le même nom.

La complanata type est, pour moi, l'espèce décrite en 1833 et représentée figure 68 par Rossmässler dans le premier fascicule de son iconographie. Cette complanata est une forme assez allongée, bien rostrée, à partie antéricure arrondie très réduite.

Quant à cette complanata du même auteur publiéc (fig. 283) en 1836 dans le même ouvrage, elle est, à mon avis, une forme nouvelle ( $P$ s. Rossmässleri) très reconnaissable à ses valves ovato-oblongues et à ses stries ellipsiformes. Il faut rapporter à celte espèce la figure 3 de la planche XLV de l'Histoire des Mollusques de France, de Moquin-Tandon. Cette figure, étiquetée complanata, est une reproduction peu heureuse de la figure 283 de Rossmässler.

L'espèce représentée sous l'appellation de complanata à la planche 3 (fig. 1) des "Eludes sur les Naïades de France» (18ä4), ne ressemble point, à mon sens, à aucune de celles que je viens de nommer. Je ne sais même à quelle 
forme on pourrait rapporter cette bivalve que je ne possède point.

La complanata (fig. 77) de Nordenskiöld et Nylander (Finlands Moll.) me paraît une bonne espèce, distincte aussi bien de la complanata (fig. 68) de Rossmässler, que de toutes les autres du même nom. Cette forme, spéciale au nord de la Russie, mérite d'ètre conservée sous l'appellation de Nordenskiöldi.

Enfin, quant aux nombreuses complanala si grossièrement représentées (fig. 16ว0-รั4 et 16ə̋6-57) dans la Suite à l'Iconographie de Rossmässler, j'ignore complètement ce qu'elles peuvent être.

Les Pseudanodontes se groupent, d'après leur forme extérieure, en quatre séries.

$1^{\circ}$ En espèces subtétragones, subarrondies, ovalaires ou subtriangulaires (quelques-unes de ces Pseudanodontes, ainsi que celles de la série suivante, ressemblent extérieurement à des Pseudodon).

Pseud. Præclara, Letourneux.

- Letourneuxi, Bourguignat.

- Danubialis, id.

- Penchinati, id.

- mecyna, Letourneux.

- complanata (Anod.), Ziegler.

- Grateloupiana (Gratelupeana), Gassies.

- Normandi, Dupuy.

$2^{\circ}$ En espèces pesantes, à test assez épais, à partie postérieure, depuis l'angle postéro-dorsal jusqu'au rostre, comme tronquée. 
Pseud. Pancici, Letourneux.

- scrupea, Bourguignat.

- Berlani, id.

$3^{\circ}$ En espèces oblongues, à stries d'accroissement ellipsiformes.

Pseud. Rossmässleri, Bourguignat.

- ellipsiformis, id.

- Nordenskiöldi, id.

$4^{\circ}$ En espèces allongées, assez ventrues.

Pseud. Tanousi, Letourneux.

- Rayi, Mabille.

- Klettii (Anod.), Rossmässler.

- Elongata (Anod.), Holandre.

- et Ligerica, Servain.

\section{$\S 1$}

\section{Pseudanodonta praeclara}

Pseudanodonta præclara, Letourneux, in Litt.

Long. $\max . . \ldots \ldots \ldots \ldots \ldots \ldots, 110$ mill.

Haut. $\max . . . \ldots \ldots \ldots \ldots . . .668$ -

Epaiss. max. (à 30 mill. du sommet,

67 du rostre, 43 du bord antér.,

45 de l'angle postéro-dorsal et

38 de la base de la perpendicu-

laire)..................

$24-$

Long. de la crête ligamento-dorsale, du sommet à l'angle postérodorsal.................. $52 \quad$ 
Distance de cet angle au rostre.... $\quad \mathbf{4 4}$ mill.

Corde apico-rostrale...........99 90 -

Hauteur de la perpendiculaire..... 63 -

Dist. max. de cette perpendiculaire au bord antérieur. ...........

- - du même point de cette perpend. au rostre postérieur

- _ enfin, de la base de cette perpend., à l'angle postéro-dorsal...........

Coq. de grande taille, très aplatie pour sa grandeur, de forme un peu sublétragone, faiblement bâillante à sa partie inféro-antérieure ainsi qu'à la partie située entre l'angle postéro-dorsal et le rostre postérieur. Partie supérieure ou dorsale faiblement arquée, presque rectiligne. Partie inférieure ou palléale légèrement convexe. Région antérieure largement arrondie. Partie postérieure deux fois plus développée, large et offrant à son extrémité un angle rostral obtus. Grête postéro-dor. sale obtuse, peu comprimée, médiocrement développée. Arête dorsale faiblement convexe, à surface passant d'une façon insensible de la convexité ventrale à la crête postérodorsale. Sommets non proéminents, aplatis-comprimés (à crochets très aigus et fort petits), ornés seulement de deux ou trois zones de petits tubercules allongés dans le sens antéro-postérieur. Stries d'accroissement présentant la forme exacte du contour des valves, c'est-à-dire arrondies en avant, convexes inférieurement, un peu rostrées postérieurement, saillantes, émoussées sur la partie convexo-ventrale, feuilletées sur lout le pourtour, ainsi que sur la crête dorsale. Epiderme brillant (à l'exception MaI. 1880. 
des parties feuilletées), d'une teinte verdâtre; avec des zones plus ou moins définies, jaunacées, rougeâtres ou brunâtres. Intérieur des valves d'un blanc bleuâtre irisé, passant, vers les sommets, à un nacré rougeâtre (sur un échantillon, la nacre est uniformément d'un iris rougeâtrelie de vin). Impressions (card. et latér.) superficielles, larges en forme de palette. Impressions palléales peu profondes.

Ligament antérieur, interne, s'étendant depuis l'angle antéro-supérieur jusqu'à 14 mill. en arrière des crochets, en occupant en largeur toute l'épaisseur de la charnière, et terminé par une toute petite lunule qui ne déborde pas en dehors de la charnière. - Ligament postérieur, externe, fort, robuste, noir (symphynoté dans le jeune âge, à découvert dans l'âge adulte), s'étendant des crochets à 10 mill. en avant de l'angle postéro-dorsal, où il se.termine à l'intérieur par une longue lunule d'un nacré noir-marron, faisant saillie sur l'extrémité de la charnière latérale.

Charnière cardinale, d'une longueur de 37 mill., présentant sur les deux valves, un peu en arrière des crochets, une dépression marron résultant d'un renflement du ligament interne, et, à chaque extrémité de cette dépression, aussi bien en avant qu'en arrière, une lamelle filiforme d'une extrême ténuité, d'un nacré blanchâtre. - Charnière latérale représentée par un renflement plan, allongé (long. 26 mill.), d'une teinte mate, se terminant d'une façon brusque à la lunule du ligament, tout en se poursuivant en dessous jusqu'à l'angle postéro-dorsal sous l'apparence d'un léger filet blanchâtre.

A l'état jeune, la prxclara possède un bord supérieur rectiligne, une partie antérieure moins largement arrondie et un rostre postérieur un tant soit peu plus aigu. 
Celte belle espèce a été recueillie par le conseiller Letourneux, sur un fond vaseux du Danube, près de Giurgewo (Valachie).

\section{Pseudanodonta Letourneuxi}

Long. $\max . \ldots \ldots \ldots . \ldots . \ldots . . . .67$ mill.

Haut. $\max . . . \ldots \ldots \ldots \ldots \ldots .66$.

Epaiss. $\max$ (à 27 mill. en arrière et audessous du sommet, à 56 du rostre, 43 du bord antér., 32 de l'angle postéro-dorsal, 42 de la base de la perpendiculaire) .............

Longueur de la crête ligamento-dorsale, du sommet à l'angle postéro-dorsal. 43 Distance de cet angle au rostre..... 40 1/2 Corde apico-rostrale............ 79 Hauteur de la perpendiculaire..... 60 1/2 Dist. max. De cette perpend. au bord antérieur.......... 29 -

- - du même point de cette perpend., au rostre postérieur..............

- - enfin, de la base de cette perpend, à l'angle postéro-dorsal. ..........

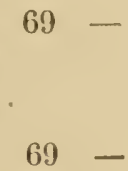

Coq. d'assez' grande taille, très aplatie, d'une convexité régulière et de forme ovalaire, bâillante dans toutes ses parties antérieure et inférieure, ainsi qu'un tant soit peu au-dessus de l'angle postéro-dorsal, - bord supérieur légèrement convexe; partie antérieure très raccourcie, 
arrondie; bord inférieur très dilaté, fortement arquéarrondi; partie postérieure plus de deux fois plus développée que l'antérieure, assez allongée et terminée par un rostre obtus à peu près médian. Crête postéro-dorsale obtuse, à peine saillante, terminée par un angle peu sensible, tant qu'il est obtus. Crête dorsale nulle. Sommets aplatis, comprimés (à crochets très aigus), ornés de trois à quatre pelites éminences tuberculeuses. - Stries d'accroissement fines et serrées au sommet, émoussées sur la convexité, feuilletées seulement au bord antérieur et au bord postérieur depuis le sommet jusqu'au rostre. Épiderme brillant, à l'exception des parties feuilletées, d'une teinte verdâtre avec des zones jaunâtres. Sommets d'une teinte brunâtre s'éclaircissant vers les crochets. - Intérieur d'une belle nacre irisée bleuâtre avec des nuances couleur de chair vers les sommets.

Ligament antérieur, interne, s'étendant depuis l'angle antéro-dorsal jusqu'à 9 mill. en arrière des crochets, en occupant toute l'épaisseur de la charnière et terminé par une lunule débordant, sur le revers intérieur de la charnière de la valve droite. Ligament postérieur, externe, robuste, épais, noir, s'étendant des crochets jusqu'à 10 mill. en avant de l'angle postéro-dorsal, où il se termine par une longue lunule, échancrant, comme chez la proclara, l'extrémité de la charnière latérale.

Charnière cardinale (long. de 20 mill.) complètement nulle, présentant seulement à son extrémité un léger renflement obtusement subtriangulaire, ne formant pas saillie, mais dû uniquement au debord de la lunule. - Charnière latérale représentée par un renflement plan, étroit, allongé (long. 26 mill.), se poursuivant au-dessous de la lunule sous la forme d'un pelit filet blanc. 
Cette Pseudanodonte, que je me fais un plaisir de dédier à notre ami le conseiller Letourneux, vit dans le Danube, où elle a été recueillie dans les environs de Giurgewo.

Cette espèce se distingue surtout par sa partie antéricure rétrécie, et par la grande dilatation arrondie de son bord postérieur. Chez elle, le rostre postérieur, situé plus haut, s'allonge presque à la partie moyenne, tandis que celui de la prxclara est plus inférieur. Ses lignes d'accroissement, ellipsiformes, modelées sur le contour général des valves, sont aussi toutes différentes de celles de la præclara.

\section{Pseudanodonta Danubialis.}

Long. $\max \ldots \ldots \ldots \ldots \ldots \ldots \ldots . . .98$ mill.

Haut. $\max . . . \ldots \ldots \ldots \ldots \ldots$ f $1 / 2$

Epaiss. max. (à 28 en arrière et en dessous des crochets, 54 du rostre, 43 du bord antér., 36 de l'angle postéro-dorsal et à 34 de la base de la perpendiculaire)............

Long. de la crête ligamento-dorsal, des crochets à l'angle postéro-dorsal.

Dist. de cet angle au rostre........ 38 -

Corde apico-rostrale........... $79 \quad$ -

Haut. de la perpendiculaire....... 57 -

Dist. max. de cette perpend. au bord antérieur......... 29 -

- -. du mème point de cette perpend., au rostre postérieur........... 68 
Enfin, dist. max. de la base de cette perpend. à l'angle postéro-dorsal......... 62 mill.

Coq. de forme oblongue, bâillante dans tout son contour antérieur et inférieur ainsi qu'entre l'angle postérodorsal et le rostre, remarquable par la convexité régulière el assez forte de ses valves. Bord supérieur faiblement convexe, un peu rectiligne. Partie antérieure bien arrondic. Bord inférieur régulièrement convexe. Partie. postérieure deux fois plus développée que l'antéricure, allongée, terminée par un rostre obtus assez inférieur.

Crête ligamento-dorsale obtuse, peu saillante. Angle postéro-dorsal très obtus. Arête dorsale insensible, sauf vers le rostre. Sommets convexes (à crochets très aigus), très finement striés.

Stries d'accroissement émoussés sur la convexité, feuilletés vers le bord antérieur, ainsi que vers la région de la crête ligamento-dorsale et vers le rostre. Épiderme brillant (sauf sur la parlie feuilletée), d'une belle teinte verte légèrement radiée de rayons plus foncés, à l'exception des sommets teintés d'une couleur grise-cendrée, plus pâle vers les crochets. Intérieur des valves d'une nacre blanchebleuâtre.

Ligament antérieur, interne, s'étendant de l'angle antérodorsal jusqu'à 10 mill. au delà des crochets, en occupant toute l'épaisseur (surtout en avant) de la charnière et terminée par une lunule insensible.

Ligament postérieur, externe, fort saillant, se terminant à 10 mill. en avant de l'angle postéro-dorsal par une grande lunule échancrant la charnière latérale.

Charnière cardinale (long. 26 mill.) complètement envahie par le ligament antéro-interne et n'offrant aucune 
ondulation. Charnière latérale étroite (long. 25 mill.) plane, fortement échancrée à son extrémité par la lunule.

Cette espèce paraît abondante dans le bas Danube, aux environs d'Ibrailla (Berlan), de Giurgewo (Letourneux).

A l'état jeune (chez quelques échantillons), la partie antérieure seule est verdâtre, tandis que la partie médiane est d'un beaú jaune passant insensiblement vers la région postéro-dorsale et rostrale à une teinte brune-vineuse.

\section{Pseudanodonta Penchinati.}

Alasmodonta Penchinati, Bourguignat, Faune Malac., bas Danube, in Ann. malac., 1, p. 71, pl. Iv, f. 1-4, 1870.

Pseudanodonta Penchinati, Bourguignat, Classif. moll. syst. europ. p. 5็, 1877.

Long. $\max . . . \ldots \ldots \ldots \ldots \ldots . .69$ mill.

Haut. $\max . . . \ldots \ldots \ldots \ldots \ldots .63$ -

Épaiss. max. (à 33 en arrière et en dessous des crochets, 51 du rostre, 47 du bord antérieur, 32 de l'angle postéro-dorsal, 35 de la base de la perpendiculaire..............

Long. de la crête ligamento-dorsale, du sommet à l'angle postérodorsal.................. 45 -

Dist. de cet angle au rostre....... $48 \quad$ - 
Corde apico-rostrale........... 82 mill.

Haut. de la perpendiculaire........ 5ร -

Dist. de cette perpendiculaire au bord antérieur............... 26 1/2

— du même point de cette perpend. au rostre................ 72 -

- Enfin, de la base de cette perpend. à l'angle postéro-dorsal....

J'ai donné, en $\mathbf{1 8 7 0}$, la description et la représentation exacte de cette espèce. J'ajouterai seulement quelques mots sur ses caractères.

Ligament antéro-interne très épais, occupant toute la région cardinale et dépassant de beaucoup les crochets.

Charnière cardinale (long. 28 mill.) offrant d'abord une petite dent lamelliforme, puis, un peu en arrière des crochets, une forte dépression causée par l'envahissement du ligament antéro-interne, enfin, un renflement lamellaire qui se poursuit sur la latérale.

Cette pseudanodonte, caractérisée par sa partie antérieure écourtée et par l'énorme développement de sa partie postérieure, est commune dans le Danube, où elle a été trouvée à Ibrailla par Berlan, à Giurgewo et Belgrade par notre ami le conseiller Letourneux.

\section{Pseudanodonta Mecyna.}

Pseudanodonta mecyna, Letoumeux, in Litt.

Long. max............... 103 mill.

Haut. $\max . \ldots \ldots \ldots \ldots \ldots \ldots, \quad 59 \quad-$ 
Épaiss. max. (à 28 en arrière et audessous des crochets, -57 du rostre, $-46 \mathrm{du}$ bord antérieur, - 32 de l'angle [postéro-dorsa], $371 / 2$ de la base de la perpendiculaire $). . . \ldots \ldots \ldots \ldots \ldots$.

20 mill.

Long. de la crête ligamento-dorsale, du sommet à l'angle postérodorsal ....................

Distance de cet angle au rostre......

Corde apico-rostrale.............

Haut. de la perpendiculaire........

Dist. de cette perpend. au bord antérieur...................

- Du même point de cette perpend.

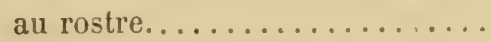

- Enfin, de la base de cette perpend. à l'angle postéro-dorsal. .

$49 \quad-$

$43-$

$84-$

$53-$

$27-$

$76-$

$68-$

Coq. très oblongue-allongée, très comprimée, presque plate (20 mill. d'épaiss. seulement sur une longueur de 103〉, faiblement bâillante à la partie inféro-antérieure, ainsi qu'à la partie postéro-dorsale.

Bord supérieur légèrement arqué. Partie antérieure bien arrondie. Bord inférieur convexe. Parlie postérieure très développée, allongée (plus de deux fois plus grande que l'antérieure), terminée par un rostre très obtus. Crête postéro-dorsale peu dilatée, comprimée. - Arête dorsale peu sensible. Sommets aplatis, comprimés (à crochets très aigus), très finement striés. - Stries d'accroissement de la forme du contour, très émoussées, un peu feuilletées en avant et sur la région postéro-dorsale. Épiderme très brillant (sauf les parties feuilletées), d'une belle teinte jaune- 
verdâtre (brunâtre à la région des sommets, ochracée vers les crochets), avec de fines radiations vertes. - Intérieur d'une nacre bleu-rougeâtre bien irisée.

Ligament antérieur, interne, filiforme, dépassant de 9 à 10 mill. les crochets et se terminant sur la latérale par une lunule excessivement exiguë.

Ligament postérieur, externe (presque entièrement symphynoté même dans l'âge adulte), robuste, obtus, terminé à l'extrémité de la latérale à 14 mill. en avant de l'angle postéro-dorsal, par une large lunule d'un nacré marron.

Charnière cardinale (long. 30 mill.) excessivement longue, tout à fait filiforme, avec une légère ondulation vers la partie lunulaire.

Charnière latérale, très longue (longueur 30 mill.), étroite, plane, horizontale, fortement échancrée à son extrémité postérieure par la lunule du ligament et se poursuivant en dessous de la lunule presque jusqu'à l'angle postéro-dorsal sous l'apparence d'un petit filet blanchâtre.

A l'état jeune, la mecyna paraît un tant soit peu plus allongée, et ses bơrds supérieur et inférieur sont un peu parailèles.

Cetle espèce, facile à distinguer par sa forme oblongueallongée, à valves excessivement plates, a été recueillie dans le Danube, aux environs de Giurgewo (Letourneux).

\section{Pseudanodonta complanata.}

Anodonta complanata, Ziegler, in Rossmëssler, Iconogr. 1, 1833̈, p. 112, f. 68 (non, complanata, Rossmässler, fig. 283. - Nec, complanata des autres auteurs). 
Pseudanodonta complanala, Bourguignat, Class. moll. syst. europ., p. 5วั, 1877.

Long. $\max . \ldots \ldots \ldots \ldots, 68$ mill. 82 mill.

Haut. $\max . \ldots \ldots \ldots \ldots .34-47-$

Épaiss. $\max \ldots \ldots \ldots \ldots 18-201-$

Distance de la crête liga-

mento-dorsale, du som-

met à l'angle postéro-

dorsal...........34 $3437-$

Distance de cet angle au

rostre............ $32-35-$

Corde apico-rostrale. . ... 58 - 66 -

Haut. de la perpendiculaire. $27-43-$

Dist. max. de cette perpend.

au bord antérieur.... $14-23$

- du même point de cette perpend., au rostre... 53 - $58 \ldots$

- enfin, de la base de cette perpend., à l'angle postéro-dorsal...... $42-54-$

J'aidonné une double mensuration, d'après deux échantillons, l'un provenant du Danube à Vienne, où a été signalé le type, l'autre du Danube à Belgrade.

Le premier est identique comme taille, comme forme à l'échantillon figuré par Rossmässler. Le second, un peu plus grand, conserve avec le premier un rapport constant de mesures. - Ils ont, du reste, tous les deux les mêmes contours et la même apparence.

1. A 23 du sommet, -46 du rostre, -39 du bord antérieur, 26 de l'angle postéro-dorsal, et 29 de la base de la perpendiculaire 
L'échantillon type, représenté figure 68, par Rossmässler, suffit amplement pour la connaissance de l'espèce. Cette figure est excellente et très exacte. Elle montre une coquille, en forme de fer de lance, caractérisée par une partie antérieure écourtée, à contour arrondi; par une partie postérieure (plus de 2 deux fois plus longue) dilatée, bien rostrée, par une arête dorsale émoussée, un peu convexe, par une crête dorsale peu élevée, terminée par un angle obtus, bien accentué, etc.

Chez cette espèce, le bord inféricur est bien convexe, tandis que le bord supérieur, peu convexe, est même un tant soit peu rectiligne. D'après la figure 68 de Rossmässler, ce bord paraît convexe-ascendant. Cette apparence de caractère résulte de la fausse position de l'échantillon figuré. Rossmässler, en représentant le bord antérieur et le rostre de la complanata, sur le même plan horizontal, a donné nécessairement à toute la partie supérieure une forme convexe très bombée. Mais si, à mon sens, il avait disposé (comme toutes les bivalves doivent l'être) le bord supérieur, qui est la partie principale (puisqu'elle renferme les ligaments, la charnière, les sommets), sur un plan horizontal, c'est-à-dire les deux extrémités du bord supérieur sur le même plan, il aurait obtenu une forme diffésente. Le bord supéricur serait devenu à peine convexe et le rostreserait incliné en bas au lieu d'être au niveau de l'horizon.

Valves très bâillantes sur tout le conlour antéro-postérieur et à peine entr'ouvertes au-dessous de l'angle postérodorsal. Stries présentant, comme contour, la forme exacte des valves, très émoussées, feuilletées seulement au bord antéro-inférieur et sur la crête dorsale. Épiderme brillant, verdâtre avec des zones jaunâtres. Intérieur d'une nacre irisée blanche-bleuacée. 
Ligament antérieur, interne, s'étendant depuis l'angle antéro-supérieur jusqu'à 6 mill. au delà des crochets, occupant, surtout vers sa partie médiane, toute l'épaisseur - de la charnière et venant s'évanouir sur la latérale d'une façon insensible. - Ligament postérieur, externe, robuste, d'une teinte marron, se poursuivant des crochets jusqu'à כ̃ mill. de l'angle postéro-dorsal, où il s'épanouit sous la forme d'une lunule subtétragone.

Charnière cardinale (long. 20 mill.) présentant sur la valve dextre une dépression ondulée très allongée, et, sur la valve sénestre, une arête filiforme très étendue. Gharnière latérale, mince, plane, horizontale, profondément échancrée à son extrémité par la lunule du ligament postérieur.

Cette Pseudanodonte ne m'esi connue que du Danube où elle vit aux environs de Vienne (Ziegler), de Belgrade (Letourneux). Elle a été signalée dans un grand nombre de fleuves d'Allemagne, mais je doute fort que les individus de ces divers cours d'eau soient des représentants de cette espèce.

\section{Pseudanodonta Grateloupiana:}

Anodonta Gratelupeana, Gassies, Moll. Agen. p. 193, pl. 2, f. 13 et 14 seulement, et pl. 3, f. 1-3 et pl. 4, f. 2, 1849. - Dupuy, Cat. ext. Gall. test. .n ${ }^{\circ}$ 16, 1849,

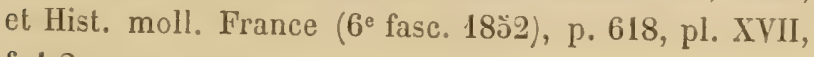
f. 1-2.

- Pseudanodonta Gratelupeana. Bourguignat, Class. moll. syst. europ. p. 5̌. 1877.

Cette espèce est parfaitement représentée dans l'ouvrage 
de Gassies. Comme je ne possède pas cette bivalve, je ne puis donner la mensuration de cette coquille que d'après la gravure de l'auteur des Mollusques de l'Agenais.

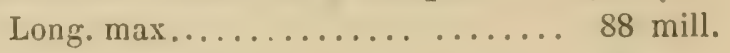

Haut. $\max . . . \ldots \ldots \ldots \ldots \ldots$....... 50 -

Epaiss. $\max . \ldots \ldots \ldots \ldots \ldots \ldots 20$ -

Long. de la crête ligamento-dorsale, du sommet à l'angle postéro-dorsal. 40 -

Dist. de cet angle au rostre........ 40 -

Corde apico-rostrale.......... 70 -

Haut. de la perpendiculaire........ 38 -

Dist. max. de cette perpend. au bord antér.................... 22 -

- du même point de cette perpend., au rostre................. 65 -

- enfin de la base de cette perpend., à l'angle postéro-dorsal.......... 58 -

Cette belle espèce, de forme subtriangulaire, est très bâillante (Gassies, pl. 4, f. 2) à sa partie antéro-inférieure, ainsi qu'à son bord postéro-dorsal. La partie antérieure arrondie est très écourtée ; enfin, le rostre est obtus et arrondi.

La Grateloupiana vit dans la Garonne aux environs de Beauregard, de Rouquet (Lot-et-Garonne). L'abbé Dupuy et deux ou trois autres auteurs ont signalé cette espèce dans la Loire, la Seine, etc. Je doute fort que les échantilIons de ces fleuves soient de vraies Grateloupiana.

Gassies a fait figurer (pl. 2, fig. 1ั̆-16) et a mentionné sous le nom de var. B. globosa une forme de la Garonne, qui me paraît différer de la Grateloupiana.

Cette forme offre une convexité de 2õ mill. sur une longueur de 72 et une hauteur maximum de $\mathbf{4 5}$, tandis que le 
type ne possède que 20 sur 88 de longueur et 50 de hauteur. Cette variété est relativement beaucoup plus haute par rapport à la longueur; la partie postérieure est moins allongée, le rostre plus arrondi, le bord inférieur plus convexe, etc. Je crois que cette variété de la Garonne, si elle est reconnue constante, doit constituer une forme à part, sous le nom de Pseud. globosa.

\section{Pseudanodonta Normandi}

Anodonta Normandi, Dupuy, Cat. extr. Gall, test. $\mathrm{n}^{\circ} 21$, 1849 , et Hist. moll. France ( $6^{\mathrm{me}}$ fasc. 185̃2), p. 620, pl. XVI, f. I0ั.

Cette coquille est bien représentée dans l'ouvrage de l'abbé Dupuy. Elle vit dans l'Escaut à Valenciennes, et aux environs d'Abbeville dans la Somme. Je la possède de la Noë, dans le Calvados. Malheureusement mes échantillons ne sont pas tout à fait adultes, ce qui m'empêche de donner une description détaillée de cette espèce qui me paraît très différente de la complanata ou de la Grateloupiana, avec lesquelles elle a été confondue par plusieurs auteurs.

Chez cette espèce (d'après mes échantillons), le ligament antéro-interne est tout à fait filiforme. La charnière offre (sur les deux valves), un peu en arrière des crochets, une petite lamelle très mince et peu élevée. Les valves sont allongées, un peu en forme de fer de lance. La plus grande hauteur se trouve au niveau de l'angle postéro-dorsal. 
Chez les espèces de cette série, le test est épais et possède un épiderme peu brillant, marron ou noirâtre; les stries sont généralement grossières et rugueuses; enfin, (signe distinctif important) le bâillement des valves, à la partie postérieure, n'a pas lieu, comme chez les Pseudanodontes précédentes, au-dessous de l'angle postéro-dorsal, mais beaucoup plus bas, un peu au-dessus du rostre sous l'apparence d'une petile ouverture ovalaire peu allongée. Quant à l'entrebâillement de la partie antéru-inférieure, il est médiocre.

\section{Pseudanodonta Panciei.}

Pseudanodonta Panici. Letourneux, in litt.

Long. $\max \ldots \ldots \ldots \ldots \ldots \ldots \ldots . . \ldots 9$ mill.

Haut. $\max . \ldots \ldots \ldots \ldots \ldots .63-$

Epaiss. max. (à 18 du sommet, $6 \check{d u}$ rostre, $3 \check{a} \cdot d u$ bord antér., $3 \check{\text { égale- }}$ ment de l'angle postéro-dorsal et $\mathbf{4 1}$ de la base de la perpendiculaire) . . 20 -

Long. de la crête ligamento-dorsale, du sommet à l'angle postéro-dorsal................... 49 -

Dist. de cet angle au rostre........ 46 -

Corde apico-rostrale........... 85 - 
Haut. de la perpendiculaire........ รั3 mill.

Dist. de cette perpend. au bord antérieur................... 2ö -

— du mème point de cette perpend. au rostre................. 7 o -

- enfin, de la base de cette perpend., àl'angle postéro-dorsal......... 67 -

Coquille large, ovalaire, très comprimée, aplatie. Bord supérieur assez convexe. Pạtie antérieure bien arrondie. Bord inférieur régulièrement convexe. Partie postérieure très dilatée, large, obscurément rostrée el offrant, de l'angle postéro-dorsal au rostre, un bord descendant, presque rectiligne, comme tronqué. Crête ligamentn-dorsale obtuse, peu élevée. Arête dorsale appréciable seulement vers la région dụ rostre. Sommets aplatis, comprimés, finement striolés, à crochets très aigus. Stries rugueuses, grossières, feuilletées vers les contours et notamment sur la crête dorsale. Épiderme terne, obscurément jaune-verdâtre, devenant, dans l'àge adulte, d'un ton noir-marron avec des zones plus ou moins accentuées. Nacre intérieure assez peu éclatante, blanchâtre ou orangée avec des taches grisâtres sales. Impressions profondes.

Ligament antérieur, interne, large, volumineux, occupant toute l'épaisseur de la charnière, s'étendant depuis la partie antérieure de l'impression jusqu'à 12 mill. au delà des crochets, où il se termine sans lunule sur la latérale. Ligament postérieur, externe, saillant, bombé, volumineux, d'un marron-rougeâtre a vec une grande lunule à son extrémité.

Charnière cardinale (long. 27 mill.) pourvue, sur les deux valves d'un filament ondulé, plus accentué sur la valre sénestre que sur la dextre. Charnière latérale (long.

MAI. 1880. 
30 mill.) plane, horizontale, étroite, carrément échancrée à son extrémité par le ligament.

Cette Pseudanodonte que notre ami le conseiller Letourneux a dédiée au professeur Pancic de Belgrade, vit dans le Danube aux environs de Giurgewo.

\section{Pweudanodonta serupea}

Long. $\max . \ldots \ldots \ldots \ldots \ldots \ldots \ldots . . .90$ mill.

Haut. $\max . . \ldots \ldots \ldots \ldots \ldots \ldots \ldots 57$ -

Epaiss. max. (à 23 du sommet, 6 so du rostre, 39 du bord antér., 21 de l'angle postéro-dorsal et 38 de la base de la perpendiculaire..... 23 -

Long. de la crête ligamento-dorsale, du sommet à l'angle postéro-dorsal. 36 -

Distance de cet angle au rostre..... $46 \quad$

Corde apico-rostrale........... 75 -

Haut. de la perpendiculaire....... 49 -

Dist. max. de cette perpend, au bord antéricur............... 24 -

- du même point de cette perpend. au rostre.............. 6 ö -

- enfin, de la base de cette perpend. à l'angle postéro-dorsal....... 57 -

Coq. oblongue-descendante, avec une partie rectiligne comme tronquée, de l'angle postéro-dorsal jusqu'au rostre. Valves assez convexes (convexité presque aussi prononcée depuis le point signalé à la mensuration, jusque vers la partie médiane de la partie rectiligne). Bâillement postérieur réduit à une petite fente ovalaire au-dessus 
du rostre. Bâillement antérieur, très peu ouvert, vers la région antéro-inférieure. - Bord supérieur légèrement convexe. Parlie antérieure écourtée, arrondie. Bord inférieur convexe-descendant. Partie postérieure allongée-descendante paraissant fortement rostrée par suite de la troncature du bord supéro-rostral. - Arête dorsale très accentuée vers la partie postéricure. Stries fortes et grossières. Epiderme terne, épais, d'un noir-marron uniforme, un peu plus clair vers les sommets. Nacre intérieure peu brillante, blanchâtre avec des tons irisés bleuâtres ou rougeâtres. Sommets aplatis, comprimés, toujours érosés.

Ligament antérieur, interne, excessivement puissant, très épais, d'une longueur de 38 mill., occupant presque toute l'épaisseur de la cardinale ct venant s'épanouir sur la partie moyenne de la latérale (à 20 mill. en arrière des crochets) sous la forme d'une immense lunule marron profonde, qui quelquẹfois déborde sur le revers intérieur de la latérale, en simulant une impression. Ligament postérieur, externe, très volumincux, fort saillant, terminé à 6 mill. en avant de l'angle postérodorsal par une forte lunule, moins développée, cependant; que celle du ligament interne.

Charnière cardinale très longue, réduite par suite du rolume du ligament interne, à une lamelle filiforme, ondulée, présentant en avant de la lunule un renffement accentué. Charnière latérale excessivement courte (long. 11 mill.), par suite de l'envahissement du ligament interne, et offrant une surface plane et horizontale.

Le Bas-Danube, aux environs de Giurgewo (Letourneux).

Cette espèce est très caractérisée par sa troncature, par 
son volumineux ligament antéro-interne et par l'exiguité de sa latérale.

\section{Pseudanodonta Berlani}

Alasmodonta Berlani, Bourguiignat, Faune du Bas-Danube, in Ann. malac. 1, 1870, p. 72, pl. III, f. 7-8, et (tirage à part) p. 37, pl. III, f. 7-8, 1870.

Pseudanodonta Berlani. Bourguignat, Class. moll., syst. europ., p. 55 , 1877.

Long. $\max . . . \ldots \ldots \ldots \ldots \ldots . . .70$ mill.

Haut. $\max . . . \ldots \ldots \ldots \ldots \ldots . . .44-$

Epaiss. max. (à 23 des sommets, 38 du rostre, 30 1/2 du bord antér., 22 de l'angle postéro-dor-

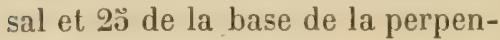
diculaire).$\ldots \ldots \ldots \ldots \ldots \ldots \ldots 17$ -

Long. de la crête ligamento-dorsale, du sommet à l'angle postérodorsal................. 34 -

Dist. de cet angle au rostre....... 32 -

Corde apico-rostrale............ 60 -

Haut. de la perpendiculaire........ 39 -

Dist. de cette perpend. au bord antér, $\quad \begin{array}{ll}16 & 1 / 2\end{array}$

— du même point de cette perpend., all rostre............... 52 -

- enfin, de la base de cette perpend., à l'angle postéro-dorsal........ 45 -

J'ai donné la description et la représentation de cette 
espèce. Je crois, cependant, devoir modifier quelque peu ma description première, à cause de la nouvelle position que je donne à mes échantillons.

Coq. de taille médiocre, comprimée, de forme ovalaire. Bord supérieur assez arqué. Partie antérieure très réduite, écourtée, arrondie. Bord inférieur convexe-descendant. Partie postérieure oblongue, dilatée, rectiligne, descendant à partir de l'angle postéro-dorsal jusqu'au rostre qui est prononcé et qui regarde en bas. Ligament antérieur, interne (long. 28 mill.), plus volumineux en arrière qu'en avant, occupant toute l'épaisseur de la charnière, se terminant (sans lunule) sur la latérale à 1 o̊ mill. en arrière des crochets. Ligament postérieur externe, fort, saillant, avec une grande lunule échancrant carrément l'extrémité de la latérale.

Charnière cardinale ondulée, avec un léger sillon filiforme, très allongé, présentant un renflement vers sa partie moyenne et une dépression à son extrémité postérieure. Charnière latérale (long. 14 mill.), mince, plane, horizontale.

Cette espèce a été recueillie par M. E. Berlan dans le Danube aux environs d'Ibraïla.

Je ne connais que trois espèces de cette série. Elles sont caractérisées par une forme oblongue, à stries ellipsiformes, par des valves minces, à peine convexes. 


\section{Pseudanodonta Rossmassleri}

Anodonta complanata, Rossmïssler, iconogr. IV, 1836, p. 24, f. 283, - et, Moquin-Tandon, moll. France, atlas, pl. XLV, f. 3 (qui est une mauvaise copie de la fig. 283 de Rossmässler - non, Anodonta complanata des autres auteurs).

Long. $\max , \ldots \ldots \ldots \ldots \ldots \ldots, 93$ mill.

Haut. $\max . . . \ldots \ldots \ldots \ldots \ldots .60$.

Épaiss. max. (à 30 des sommets, õl du rostre, 43 du bord antér., 28 de l'angle "postéro-dorsal, 33 de la base de la perpendiculaire)..... 19 -

Long. de la crête ligamento-dorsale, du sommet à l'angle postírodorsal............... 42 1/2

Dist. de cette angle au rostre...... 40 -

Corde apico-rostrale............ . $80 \quad$ _

Ilaut. de la perpendiculaire.... . . . ذ̇ _

Dist. de cette perpend. au bord anter. 2つั -.

- dı même point de cette perpend. au rostre................ 69 -

- enfin, de le base de cette perpend. à l'angle postéro-dorsal....... 61 -

Coq. tout à fait ovalaire-ellipsiforme, très comprimée, presque aplatie. Bord supérieur convexe. Partie antérieure écourlée-arrondie. Bord inférieur convexe, un peu descendant. Partie postérieure allongée-ovalaire, obtusément rostréc. Valves fort peu entrebâillées à la partie antéroinférieure et au bord postéro-dorsal. Arête pustéro-dorsale 
peu développée, obtuse. Crête dorsale peu accentuée, présentant, cependant, une ligne légèrement arquée qui des sommets aboutit à la partie rostrale supérieure. Sommets comprimes; aplatis, un peu ondulés tuberculeux, à crochets aigus. Stries saillantes, assez grossières, ellipsiformes. Épiderme assez brillant, verdâtre avec des zones jaunacées ou brunâtres d'une teinte marron sur la crête postéro-dorsale. Nacre intérieure peu brillante, blanchâtre, peu irisée.

Ligament antérieur, interne, large, occupant toute l'épaisseur de la cardinale, s'étendant sur une longueur de 30 mill. depuis l'angle antéro-dorsal jusqu' à 17 mill. en arrière des crochels el se terminant sans lunule. Ligament postérieur, externe, fort, robuste, recouvert presque toujours par le test, se poursuivant pour ainsi dire jusqu'à l'angle postéro-dorsal et pourvu à l'intérieur d'une longue lunule.

Charnière cardinale occupant toute la longueur du liga ment interne, réduite à un sillon filiforme ondulé, pourvue, en arrière de crochets, d'un renflement légèrement saillant. Charnière latérale (long. 19 mill.), plane, étroite.

Cette espèce habite dans tont le cours du Danube. Je la possède de Giurgewo, de Belgrade (Letourneux), de Vienne, etc.

\section{Pseudanodonta ellipsiformis}

Long. $\max . . . \ldots \ldots \ldots \ldots \ldots . . . . . .60$ mill.

Haut. $\max . . . . \ldots \ldots \ldots \ldots \ldots$......

Epaiss. max. (à 23 du sommet, 46 du rostre, $31 \mathrm{du}$ bord antérieur, 28 
de l'angle postéro-dorsal, 2ə̊ de la base de la perpendicul.)....... 16 mill.

Long. de la crête ligamento-dorsale, du sommet à l'angle postéro-dorsal. . 36 -

Dist. de cet angle au rostre....... 36 -

Corde apico-rostrale........... 66 -

Haut. de la perpendiculaire....... 450

Dist. de cette perpend. au bord antérieur..................

- Du même point de cette perpend. au rostre............. эั8 -

- Enfin, de la base de celte perpend. à l’angle postéro-dorsal..... ذّ -

Coq. tout à fait oblongue-allongée, excessirement plate et comprimée. Bord supérieur peu convexe. Partic anle. ricure écourtée-arrondie. Bord inférieur convexe-descendant. Partie postérieure allongée-oblongue, obtusement rostrée. Crête postéro-dorsale assez comprimée, peu développée. Arête dorsale peu accentuée. Valves très minces, rragiles, à peine entrebâillées à la partie antéro-inférieure (entrebâillement réduit à une loute petite fente), ainsi qu'à son bord postéro-dorsal. Stries très émoussées, ellipıiformes, fines, régulières, feuilleties sculement vers le bord antérieur et sur la crète postéro-dorsale. Épiderme brillant (sauf sur les parties feuilletées), d'une teinte couleur de chair vers les sommets, puis passant, vers les contou's, à une nuance jaunacéc-rougeâtre tirant sur le vert. Nacre intérieure blanchàtre, très-brillante et bien irisée.

Ligament antérieur, interne, filiforme (long. 22 mill.), sétendant en arrière des crochets de 11 mill., sans lunule appréciable à son extrémité. Ligament postérieur, externe 
(symphynoté), médiocre, ne dépassant pas le test, se lerminant à 11 mill. en avant de l'angle postéro-dorsal sous la forme d'une longue lunule, convexe à sa partie inféJieure.

Charnière cardinule très allongée, filiforme, présentant un long filament blanchâtre, non élevé, creusé au niveau du crochets et un peu relevé vers son extrémité (sur la valve sénestre, la charnière présente, à l'endroit correspondant à la dépression dextre, un renflement filiforme très allongé). Charnière Jatérale allongéc, mince, horizontale, non échancrée brusquement à. son extrémité par la lunule, mais s'amoindrissant sous la forme d'un filament blanchâtre.

Celle Pseudanodonte vit dans le Danube où elle a été recueillie notamment aux enviroas de Giurgewo.

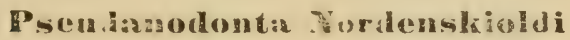

Anodonta complanata, Nordenstiveld et Nylander Finl. Moll., p. 89, pl. VII, f. 77, 1856.

Je ne connais celle forme, qui habite en Finlande, que par la courte description et par la figure qu'en ont donné Nordenskiold et Nylander. D'après cette figure, cette coquille très oralaire-allongée, à sommets situés presque en avant, à parlie postérieure très-longue et bien rostrée, à crête postéro-dorsale très accentuéc, comprimée, un tant soit peu ailée, me semble unc furme qui ne peut rentrer dans aucune de celles que je viens de signaler. 
§. 4.

Les espèces de celte série sont allongées, un peu uniopsides, à valves très-peu báillantes (sauf chez les Tanousi, Klettii et elongata. L'entrebâillement s'ouvre plus inférieurement. La plus grande hauteur chez ces Pseudanodontes se trouve portée très en arrière presque au niveau de l'extrémité du ligament postéro-externe.

\section{Pseudanodonta 'Tanousi}

Pscudanodonta Tanousi, Letorrnerux in litt.

Long. $\max . . \ldots \ldots \ldots \ldots \ldots \ldots$.... 80 mill.

Haut. $\max . . . \ldots \ldots \ldots \ldots \ldots \ldots, 46$ -

Epaiss. max. (à 18 des crochets, $50 \mathrm{du}$ rostre, 32 du bord antér. -29 de l'angle postéro-dorsal et 28 de la base de la perpendiculaire)..... $19 \quad-$

Long. de la crête ligamento-dorsal, du sommet à l'angle postéro-dorsal.. 40 -

Dist. de cet angle au rostre......... 350

Corde apico-rostrale............ $67 \quad$

Haut. de la perpendiculaire........ 41 -

Dist. de cette perpend. au bord anter.. 21 -

- du même point de celte perpend. au rostre............. 60 -

- enfin, de la base de celte perpendicul., à l'angle postéro-dorsal. 54

Coq. allongée, un tant soit peu subtétragone, haute pour sa longueur, à bords supérieur et inférieur un peu pa- 
rallèles, bien que le bord inférieur soit légèrement co.ıvexe. Valves assez aplaties, comprimées (la convexilé se poursuit assez loin sur la partie postérieure. - Bord supérieur presque rectiligne. - Partie antérieure arrondie, écourtée. Bord inférieur faiblement convexe. Partie postérieure allongée, terminée par un rostre très-obtus, assez inférieur. Crête postéro-dorsale peu élevée, obtuse. - Arête dorsale sensible, marquée par un sillon. Stries émoussées sur la convexité, grossières vers les contours, feuilletées à la partie postéro-dorsale située entre le rostre et l'angle postérieur. Épiderme brillant, d'une teinte jaunacée à la région des sommets, verdâtre avec quelques zones plus foncées sur le reste des valves. Narre intérieure d'un blanc bleuâtre irisé. Sommets aplatis, comprimés, légèrement tuberculeux avec des crochets aigus.

Ligament antérieur, interne (long. 20 mill.), sans lunule terminale, occupant presque toute l'épaisseur de la cardinale. Ligament postérieur, externe (recouvert à l'état jeune), assez volumineux, se terminant à 9 millim. en avant de l'angle postéro-dorsal et pourvu d'une lunule allongée.

Charnière cardinale (de la longueur du ligament antérointerne), filiforme, un peu ondulée avec un renflement très émoussé en arrière des crochets. Charnière latérale (long. 24 mill.), plane, horizontale, augmentant en largeur vers son extrémité.

Dans le Danube aux environs de Giurgewo (Letourneux.)

\section{Pseudanodonta Rayi}

Pseudanodonta Rayi. Mabille in litt.

Long. $\max \ldots \ldots \ldots \ldots \ldots \ldots$ tö mill. 
Haut. $\max . . . \ldots \ldots \ldots \ldots . . . . . .$.

44 mill.

Epaiss. max. (à 22 du sommet, $40 \mathrm{du}$ rostre, 3 s̆ du bord antér. - 21 de l'angle postéro-dorsal et 20 de la base de la perpendicul.)......... Long. de la crête ligamento-dorsale, du sommet à l'angle postéro-dorsal. 37 Dist. de cet angle au rostre......... 32 Corde apico-rostrale........... 61 Haut. de la perpendiculaire........ 32 Dist. de cetle perpendiculaire au bord anterieur.............

- du meme pinint de ectle perp. au rostre................

- enfin, de la base de cette perpiend. a l'angle postéro-dorsal ........ $46 \quad-$

Coq. oblongue-allongée, assez haute pour sa hauteur, à valves à peine entr'ouvertes et peu convexes (convexité presque médiane.) - Bord supérieur légèrement convexe. (Dans le jeune àge, il est presque rectiligne.) Partie antérieure arrondie. Bord inférieur assez convexe. Partie postérieure allongée, obtusement rostrée. Crête postéro dorsale obtuse, émoussée. Arête dorsale marquée par un sillon. Sommets comprimés, finement striés, tuberculeux vers les crochets qui sont fort aigus. Striés émoussées, grossières ver's les contours, rugueuses ver's la partie antéro-inféricure et feuilletées vers la région postéro-dorsale de l'angle au rostre. Épiderme jaunacé ver's les sommets, puis d'un beau vert très-brillant, avec des zones jaunes sur le reste des valves. (Dans l'étal de vieillesse, l'épiderme prend une teinte terne noirâtre uniforme). Nacre intérieure d'un blanc irisé, avec des tons orangés vers les sommets. 
Ligament antérieur, interne (long. 2つ̣ mill.) sans lunule terminale, mince, devenant, chez les vieux échantillons, épais au point d'envahir toute la cardinale. Ligament postérieur, externe (recouvert chez les jeunes), assez volumineux, se terminant à 7 mill. en avant de l'angle postérodorsal et pourvu d'une lunule qui échancre carrément la latérale.

Charnière cardinale excessivement mince, chez les jeunes, représentée par un filament blanchâtre, légèrement relevé au niveau des crochets, et, chez les vieux échantillons, presque totalement absorbée par le ligament. Charnière latérale courte, plane, horizontale.

Cette espèce, dédiée à M. J. Ray, conservateur du musée de Troyes, a été recueillie dans les bassins de Marlyle-Roy, près Paris (Mabille.)

\section{Pseudunodonta Irlettii}

Anodonta Klettii, Rossmässler, iconogr. 1. 183ä. p. 112. (sans description.)

- $\quad$ - Scholtz, Schlefiien's land und wass. Moll. p. 122. 1843, et suppl. p. 1 ö. 1853.

- - Mürch, Syn. moll. terr. et fluv. Danix p. 90. 1864.

- rhomboidea, Schlïter, Kurzg. syst. verz. Conch. Halle, p. 32 (sans descript.) 1838. (teste Mörch. 1864).

Pseudanodonta Klettii, Bourguignat, Class. moll. syst. europ. p. รั. 1877.

Je rapporte à la Klettii, l'Anodonta minima (non A. minima de Millet) de Joba (cat. Moll. Mos. p. 14 pl. un. 
1844), et, cette même coquille rééditée par Joba (suppl. cat. moll. Mos. p. 6. 18วّ1) sous le nom d'Anod. elongala (non An, elongata de Holandre 1836.)

Long. $\max \ldots \ldots \ldots \ldots \ldots \ldots \ldots$......

Haut $\max . . . \ldots \ldots \ldots \ldots \ldots . . . .$.

Epaiss. max. (à 24 des crochets, 31 du rostre, 34 du bord antér. -17 de l'angle postéro-dorsal, et 23 de la base perpendiculaire...........

Long. de la crête ligamento-dorsale, du sommet à l'angle postéro-dorsal................... 38 -

Dist. de cet angle au rostre...... 3 ö -

Corde apico-rostrale........... 5 5 -

Haut. de la perpendiculaire........ 32 -

Dist. de cette perp. au bord antérieur. 16

- du même point de celte perpend. au rostre............... 49 -

- enfin, de la base de cette perpend., à l'angle postéro-dorsal....... 40 -

Coq. de petite taille, plus haute en arrière qu'en avant (la plus grande hauteur se trouve juste au niveau de l'extrémilé du ligament postéro-dorsal), de forme allongée, avec une partie postérieure obliquement descendante. Bord supérieur légèrement convexe. Partie antéricure écourtée, arrondic. Bord inférieur presque rectiligne, descendant. Parlie postérieure très déreloppée, trois fois plus longue que l'antérieure, à bord postéro-dorsal recliligne, descendant sur le rostre qui est très obtus. Valres très bâillantes à la partie antéro-inférieure et un peu moins entre l'angle postéro-dorsal et le rostre. Convexilé (19 mill.) très prononcée, dont le point le plus saillant se 
trouve presque sur la perpendiculaire tombant de l'extrémité du ligament postérieur. Sommets assez renflés, bien que comprimés (à crochets aigus), presque toujours érosés. Crête dorsale peu élevée, émoussée, obtuse. Arête dorsale assez accentuée, légèrement arquée. Stries ordinairement grossières, émoussées sur la convexité, rugueuses aux extrémités. Épiderme peu brillant, d'un ton verdâtreobscur avec quelques zones jaunacées, mais le plus souvent d'un brun-verdâtre cendré uniforme. Nacre intérieure d'un blanc-bleuacé avec quelques nuances orangées vers les sommets.

Ligament antérieur, interne (long. 18 mill.), occupant toute l'épaisseur de la cardinale (sauf au niveau des crochets), même débordant à l'intérieur et se terminant à 9 mill. en arrière des crochets sous la forme d'une grande lunule tachant d'une teinte marron l'épaisseur intérieure et horizontale de la latérale. Ligament postérieur, externe (recouvert dans le jeune âge), fort, robuste, allongé, terminé à 6 mill. de l'angle postéro-dorsal par une grande lunule quadrangulaire qui échancre carrément l'extrémité de la latérale.

Charnière cardinale présentant au niveau des crochets une éminence filiforme, allongée, très proéminente. Charnière latérale allongée, étroite, plane et horizontale.

Cette Pseudanodonte parait commune dans toutes les eaux du nord de l'Europe depuis la France jusqu'en Russie. Je la possède notamment du Danemarck d'où elle m’a été envoyée par Mörch. 


\section{Pseudanodonta Elongata}

Anodonta elongata ", Holandre, Faune Moselle, Moll. p. $54,1836$.

Anodonta Jobæ, Dupuy, Cat. extr., Gall. test. no 18, 1849.

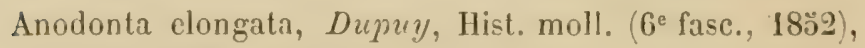
p. 620, pl. XVI, f. 16.

Pseudanodonta elongala, Bourguignal, Class. moll. syst. Europ., p. อิวั, 1877.

L'échantillon représenté fig. 2, pl. III des Etudes sur les naïades de l'Aube, n'est pas typique, il est trop large pour sa longueur.

Long. $\max . \ldots \ldots \ldots \ldots \ldots . . \ldots .68$ mill.

Haut. $\max . . . \ldots \ldots \ldots \ldots \ldots . . . .$.

Epaiss. max. (à 24 des crochets, $35 ̃$ du rostre, 31 du bord antér., 21 de l'angle postéro-dorsal el 20 de la base de la perpendiculaire)..... 20 -

Long. de la crête ligamento-dorsale, du sommet à l'angle postéro-dorsal. 38 -

Dist. de cet angle au rostre....... 27 1/2

Corde apico-rostrale........... 59 -

IIaut. de la perpendiculaire........ 28 -

Dist. de cette perpend. au bord antér. 17 -

- du même point de cette perpend. au rostre...............

1. Non., Anorl. clonguta du supplém. de Joba, que je rapporte la Klettii. 
Dist. enfin, de labase de cette perpend.

$$
\text { à l'angle postéro-dorsal..... } 42 \text { mill. }
$$

Coq. très allongée, à bords supérieur et inférieur, un tant soit peu parallèles, bien que la plus grande hauteur se trouve à l'extrémité du ligament externe, à valves bien convexes (convexité dans le sens de la longueur, dont le point le plus saillant se trouve situé presque à égale distance des extrémités, ce qui n'a pas lieu chez l'espèce précédente). - Bord supérieur presque rectiligne, faiblement convexe. Partie antérieure arrondie, très écourtée. Bord inférieur presque rectiligne, un peu descendant, avec un simulacre de contraction vers la partie centrale. - Partie postérieure plus de trois fois plus longue que l'antérieure, assez dilatée à son extrémité et terminée par un rostre très obtus, incliné vers le bas. - Valves très bâillantes à la partie antéro-inférieure (entrebâillement nul, ou presque nul, entre l'angle postéro-dorsal el le rostre), très convexes, à sommets comprimés, ondulés, un peu tuberculeux et à crochets très aigus. Stries grossières, émoussées sur la convexité. Epiderme noirâtre avec quelques zones d'un vert foncé, plus pâle vers la région des sommets. Nacre intérieure blanchâtre avec des tons bleuâtres ou orangés.

Ligament antérieur, interne, très allongé, terminé par une lunule allongée (à 10 mill. des crochets), et occupant tout l'emplacement de la cardinale, à tel point que celleci est complètement absorbée. Ligament postérieur, externe, très long, saillant, terminé à 7 mill. en avant de l'angle postéro-dorsal par une lunule triangulaire allongée, qui échancre carrément la latérale.

Charnière cardinale nulle ou presque nulle (entièrement envahie par le ligament interne), représentée chez les vieux individus par une longue concavité, et, chez les jeunes, MAI. 1880 
par un filament ondulé concave avec une légère tubérosité a son extrémité. Charnière latérale mince, très allongée, plane et horizontale.

Cette espèce habite dans la Moselle à Metz, en compagnio de la Klettii.

\section{Pseudanodonta Ligerica}

Pseudanodonta Ligerica, Servain, in Litt. et in Bourguignat, Class. moll. syst. curop. p. 55, 1877.

Long. $\max . \ldots \ldots \ldots \ldots \ldots . . .63$ mill.

Haut. $\max . \ldots \ldots \ldots \ldots \ldots \ldots . . .637$ -

Epaiss. max. (a 20 des crochets, 33 du rostre, 31 du bord antérieur, 17 de l'angle postéro-dorsal et $201 / 2$ de la base de la perpend.)...... 15 -

Long. de la crête ligamento-dorsale, dusommetàl'angle postéro-dorsal. 27 -

Distance de cet angle au rostre..... 33 -

Corde apico-rostrale........... 52 -

Ilaut. de la perpendiculaire........ $30 \quad-$

Dist. de cette perpend. au bord antér. 18 -

- Du mémo point de cette perpend. au rostre............. $46 \quad$

- Enfin, de la base de cette perpend.

à l'angle postéro-dorsal..... 38 -

Coq. allongée-subtriangulaire, très rétrécie en avant et augmentant graduellement en largeur au point que la plus grande hauteur se trouve à l'extrémité du ligament postérieur. - Convexité maximum centrale, presque à égale 
distance des extrémilés et des bords supérieur et inférieur. Bord supérieur presque rectiligne. Partie antérieure trèsécourtée, très réduite, arrondie. Bord inférieur rectiligne descendant. Partie postérieure près de 3 fois plus longue que l'antérieur, allongée, relativement très-élargie, oflrant un bord postérieur convexe et une partie inférieure obtusément rostrée, regardant en bas. Valves aplaties-comprimées, à peine bâillantes (bâillement antérieur réduit à une fente à peine ouverte sur le bord inférieur. Bâillement postérieur représenté par une toute petite ouverture allongée, peu appréciable, située au-dessus du rostre. Sommets aplatis, comprimés, toujours érosés, à crochets aigus. Stries émoussées sur la convexité, rugucuses et feuilletées vers les contours. Épiderme peu brillant, d'un brun-cendré ou marron, plus clair vers la région des sommets. Nacre intérieure d'un blanc bleuacé plus ou moins irisé.

Ligament antérieur, interne, mince, très allongé, se ter. minant sans lunule sur la latérale à 8 mill. en arrière des crochets. Ligament postérieur très saillant, pourvu d'une longue lunule terminale.

Charnière cardinale réduite à un filament très allongé, onduleux, avec un léger renflement en arrière des crochets. Charnière latérale mince, très allongée, plane et horizontale.

Cette Pseudanodonte habite dans la Loire près d'Angers (Servain): Notre ami le conseiller Letourneux a recueilli des échantillons absolumentidentiques à ceux de la Loire, dans la Save à Agram (Croatie).

M. L. de Joannis, auteur d'un très médiocre travail sur 
les naïades du département de Maine-et-Loire I, a signalé, aux environs d'Angers, trois formes de complanata, savoir la complanata (pl. 6. f. 2), la Normandi (pl.6. f. 1.) et l'elongata. - Je ne crois pas à l'exactitude des déterminations de cet auteur, parce que les deux espèces représentées ne sont ni la Normandi ni la complanata. Quant à l'elongata de Metz, je doute de sa présence à Angers. En tout cas, aucune des espèces de Joannis ne peuvent étre rapportées à la Ligerica du docteur Servain.

1. In Ann. Soc. Linn. Maine-et-Loire, III, 1859, p. 261-295 et pl. 1 à 12 . 


\title{
DESCRIPTION
}

DE PLUSIEURS

\section{ÆTHÉRIES NOUVELLES}

\author{
SUIVIE D'UN APERÇU MONOGRAPHIQUE SUR LE
}

GENRE RTHERIA

\section{Etheria Petrettinii}

Valves aussi grandes l'une que l'autre, dont l'une, adhérente (tantôt la dextre, tantôt la sénestre), complètement plate, sans rugosités et sans boursouflures extérieures.

Valve libre (ou supérieure), non feuilletée, opaque, régulièrement convexe, d'une teinte verdâtre avec des zones plus foncées et quelques bandes jaunacées plus ou moins prononcées, teinte passant versles sommets en une nuance plus claire, irisée de tons de feu. Stries concentriques, régulières, bien que rugueuses et un peu crispées (crispations parfois très accentuées).

Intérieur d'une nacre blanche, unie sur la valve supérieure pleine de tubérosités sur la valve inférieure ou adhérente. 
Partie antérieure arrondie, écourtée. Partie postérieure dilatée-arrondie.

Sommets (sans talon) petits, recourbés, opposés et pourvus à leur pointe d'une petite coquille embryonnaire. Cette coquille parait lisse et ressemble comme forme (moins les stries) à l'Arca lactea de nos côtes océaniennes.

Ligament, postérieur et interne, composé de deux parties: $1{ }^{\circ} \mathrm{d}$ 'une partie fibreuse, conique, courte, semblable comme forme au cuilleron d'une $1 / y a ; 2^{\circ}$ d'une partie ligamenteusc très étroite, située en arrière de la partie fibreuse et pénétrant profondément par une fente dans l'intérieur du test.

Charnière simple, composée: $1^{\circ}$ sur la valve libre (ou supérieure) d'un renflement pédonculé supportant le ligiment fibreux; $2^{\circ}$ sur la valve inférieure (ou adhérente), d'une série de renflements très-épais, très puissants, sinueux, ondulés, excavés en dessous au niveau des crochets et fendus à l'endroit du sillon qui contient la partie ligamenteuse.

Impression antérieure, étroite, très allongée en forme de C et fimbriée. Impression postérieure ressemblant à la forme d'un haricot. Impression palléale accentuée, irrégulièrement arrondie.

Cette espèce de forme arrondie, toujours plus longue que haute, varie beaucoup comme taille. Long. max. 50100. Haut. 40̈-90. Epaiss. 12-24 millim.

On ne peut donner, ainsi que je l'ai dit dans mon introduction, une mensuration rigoureuse des Ethéries. Les espèces de ce genre sont sujettes à trop de variations par suite de l'adhérence d'une de leurs valves à un corps étranger, sur lequel elles sont forcées de se modeler.

Cetle Æthéria, dédiée à l'ingénieur Petrettini, vit en 
Égypte, dans l'intérieur des tuyaux de fonte, ainsi qu'au fond du puisard de l'établissement des eaux de Ramlé, à Hagueret en Naouatié, non loin du canal Mahmoudieh. (Petrettini et Letourneux).

\section{Atheria Letourneuxi}

Espèce à valves des plus irrégulières, présentant toutes les variations possibles, conservant, cependant, malgré ses irrégularités, une forme transversalement oblongue, toujours plus longue que haute.

Valves de même taille (dont la supérieure très-convexe) d'une belle teinte verte et profondément sillonnées par des stries concentriques proéminentes, creusées, crispées ou boursouflées, etc. - Intérieur d'une belle nacre blanche, légèrementbleuâtre, çà et là fortementirisée, avec quelques taches livides.

Sommets (sans talon) petits, recourbés, non opposés et distants l'un de l'autre, par suite de l'encrassement et de l'épaississement de la valve adhérente; pourvus à leur extrémité d'une petite coquille embryonnaire assez semblable, comme forme, à celle de l'espèce précédente, mais en différant par un test sinueux à sa partie centro-palléale et par des stries concentriques régulières et très accentuées.

Ligament, poștérieur et à moitié interne, à cause de l'épaississement de la valve adhérente, qui par son encrassement (en laissant un espace vide entre les sommets) permet de voir en dehors une portion du ligament fibreux.

Ligament semblable, du reste, à celui de la Petrettinii, seulement s'en distinguant parla partie fibreuse plus courte et plus large. 
Charnière pédonculée sur la valre libre et des plus irregulières sur la valve adhérente.

Impressions peu prononcées: l'antérieure irrégulièrement allongée; la postérieure arquée, assez courte; la palléale sineuse-ondulée, très distante des bords marginaux.

Cette Ethérie est aussi variable dans sa taille que dans sa forme. Suivant les échantillons, elle atteint en longueur 60-90, en hauteur 40-70, en épaisseur 20-30 mill.

Cette espèce, que je me fais un plaisir de dédier à notre ami le conseiller Letourneux, vit dans le Nil et dans les canaux du Fayoum, en Egypte.

C'est d'après un échantillon de celte espèce, provenant également du Fayoum, que Rang et Caillaud (Not. g. Ethér. et desc. anim. 1834) ont pu donner l'anatomie du genre Etheria.

Seulement ces savants auteurs ont eu le tort de confondre cette forme avec la Caillaudi, qui est toute differente.

En tout cas, la planche VI sur laquelle Rang et Caillaud ont fait représenter cette espèce, donne bien les contours de cette $E$ thérie, qui, comme je l'ai dit, conserve toujours, malgré ses irrégularités, une forme plus longue que haute. C'est l'inverse chez la Caillaudi, qui, de plus, est caractérisée par un talon d'une longueur démesurée.

\section{Etheria Chambardi}

Cette espèce, très distincte des deux précédentes, a été découverte dans des couches quaternaires à Ramsès, en 
Egypte, par le docteur Chambard, atlaché aux travaux du grand canal de Suez.

Ces couches, au nombre de deux (que l'on peut appeler bancs à Ethéries, car la Caillaudi et la Chambardi y foisonnent), séparées l'une de l'autre par 3 à 4 mètres de sables un peu terreux, reposent sur un lit de cailloux roulés provenant d'une antique branche du Nil. Au-dessus de la couche supérieure à Ethéries, se trouve un dépôt de 4 à 5 mètres, dans lequel on découvre de nombreux sarcophages et une quantité considérable de momies d'anciens habitants de Ramsès.

Ces deux bancs à Ethéries sont done antérieurs à la fondation de la ville égyptienne, et les espèces qu'ils contiennent sont les représentants de l'antique faune d'une branche nilotique disparue depuis les temps les plus reculés.

L'Etheria Chambardi, d'une forme toujours oblongueallongée, malgré ses variations et ses irrégularités, est caractérisée par sa partie postérieure nulle ou presque núlle, lorsque c'est sa valve sénestre qui est adhérente, ou inversement, par sa partie antérieure réduite à fortpeu de chose, lorsque c'est sa valve dextre.

Valves de même taille, très épaisses, légères, très feuilletées, extérieurement boursouflées, irrégulièrement contournées, suivant les échantillons, et intérieurement ornées d'une nacre jaunacée. Sommets excoriés, à talons internes d'égale longueur, contournés, en suivant une direction curviligne, tantôt à droite, tantôt à gauche, et toujours recouverts par l'une et l'autre valve.

Le talon de la valve adhérente est ordinairement obliquement renversé en arrière. Quelquefois c'est celui de la valve libre. Alors, dans ce cas, le talon de la valve adhé- 
rente est ascendant, tout en se maintenant dans une direction obliquemenl dextre ou sénestre. En résumé, le talon de l'une se modèle sur celui de l'autre.

Ligament, irterne, prolongé sur toute la longueur du talon, composé, comme chez les espèces précédentes, d'une partie fibreuse et d'une autre ligamenteuse enfoncée dans une fente.

Charnière pedonculée sur la valve libre (pédoncule supportant le ligament fibreux), sinueuse-irrégulière sur la valve adhérente.

Impressions : antérieure très longue en arc de cercle; postérieure oblongue-spathuliforme; palléale irrégulière, très distante du bord marginal.

Cette espèce, comme toutes celles de ce genre, varie extrèmement dans sa taille. Long. 50-75, haut. 80-110, épaiss. $30-450$ millim.

\section{Acheria Nilotica}

\section{Etheria nilotica, Letourneux, mss.}

Cette nouvelle espèce, qui vit dans le Nil en Nubie (Joannis), ainsi que dans la haute Égypte, a élé recueillie par notre ami le conseiller Letourneux, sur les bords du lac Mariout (section orientale), près d'Alexandrie.

Les échantillons de cette localité, bien que morts, étaient intacts, sans le moindre émoussement. Celte nilotica doit vivre, par conséquent, bien près de l'endroit où elle a été trouvée.

Valves des plus irrégulières, inégales de taille (l'adhèrente un peu plus grande que l'autre), très épaisses vers 
la région supérieure où l'accroissement se fait par juxtaposition.

Épiderme d'une belle teinte uniforme, couleur feuillemorte. Nacre blanche-bleuacée et bien irisée.

Talons développés, de forme conico-pyramidale (long. 15 à 30 mill.) tournés à droite, à gauche, enfin, ascendants ou descendants. Sommet des talons très aigu.

Ligaments externes, composés comme chez les autres æthéries. Partie fibreuse, conique, largement arrondie à la base, et se modelant sur la forme du talon. Partie ligamenteuse reçue dans une fente étroite, très profonde.

Charnière très irrégulière, peu accentuée. Pédoncule presque nul. Impressions : antérieure étroite, allongée en arc de cercle; postérieure irrégulièrement spathuliforme.

Test très feuilleté. Taille des plus variables, de forme tantôt arrondie ou oblongue, tantôt allongée dans le sens transversal. Long. 70-90, haut. 60-130, épaiss. 30-50 millim.

Le genre ETHeria a été établi par Lamarck (sous le vo(cable Étheria 1, en 1807 (et non pas 1808) dans le tome X p. 398) des Annales du Museum de Paris, pour de singulières coquilles ressemblant à des huîtres.

Ce genre a été adopté par tous les auteurs.

1. . J'ai donné le nom d'Éthérie, dit Lamarck, en l'honneur de l'une des Océanides, parce que les coquilles de ce genre habitent dans la mer. - Lamarck croyait, en effet, que les Ethéries étaient marines. Ce n'est que depuis les decouvertes de Caillaud que l'on sait qu'elles sont fluviales; or, comme le nom de l'Océanide Etherie dérive de aîśftos, éthéré (à cause de la couleur bleue de la mer), ce genre doit s'écrire SETHERIA. 
Rangées d'abord dans la famille des Chames (Lamarck, 1807 et 1819), des Cardiacés (Schweigger, 1820), des $\mathrm{My}$ tilacés (Blainville, 1826), des Ostracés Dimyaires (Cuvier, 1830), etc., les Æthéries ont été placées par Deshayes, en 1830, dans une famille spéciale, celle des Etraeride.

Rang et Caillaud, en 1834, ont, les premiers, constaté, d'après les caractères anatomiques, les rapports de ce genre avec ceux des Naïades.

Voici, d'après Rang, Caillaud et Deshayes, les caractères de l'animal des Etheria'.

Animal de forme variable, revêtu d'un manteau très ample à bords désunis dans tout son contour, sauf à la région dorsale. Canal anal séparé par un point de soudure de la grande cavité palléale. Pied grand, comprimé, obtus au sommet. Bouche ovale, transverse. Palpes labiales ovales, fixées dans toute la longueur de leur bord supérieur. Deux feuillets branchiaux de chaque côté, inégaux, réunis en arrière de la masse abdominale, soudés au manteau et servant de cloison entre la cavité palléale et.le canal anal.

Coquille irrégulière, équi-ou-inéquivalve, nacrée, épidermée, à test foliacé ou non foliacé. Une des valves adhérente (tantôt la dextre, tantôt la sénestre), l'autre libre; crochets non allongés, ou, prolongés en forme de talon, possédant à leurs extrémités (lorsqu'elles sont intactes) une petite coquille embryonnaire, analogue à celle des Mulleria. Ligament interne ou externe, avec un sillon

1. Consultez: 10 Ferussac, notice sur les Ethéries trouvées dans le Nil par Caillaud, in Mém. Acad. Sc. Paris, 1823, - 20 Deshayes, Iincycl. Meth. histoire des Vers, II, 1830. - 3० Rang et Caillaud, mémoire sur le genre Ethérie et description de son animal, in Nouv. ann. mus. d'Hist. nat., III, 1834. - 40 Rang, mémoire sur quelques acephales d'eau douce du Sénégal, 1834. 
très profond à côté. Charnière sans dents, épaisse, sinueuse, sur la valve adhérente, pédonculée sur la valve libre. Deux impressions écartées (une seule chez les Mulleria), une à droite, une à gauche, réunies par une impression palléale étroite.

J'ai signalé pour la première fois, chez deux des espèces que je viens de décrire, la petite coquille embryonnaire des Æthéries.

Cette coquille (long. 3, haut. 2 mill.) libre dans le jeune âge, équivalve, à peine inéquilatérale, à ligament interne, présente une forme transversalement allongée, à extrémités arrondies, avec un sinus très prononcé vers la région centro-palléale inférieure. Sa surface est lisse (Petrettinii) bu bien striée (Letourneuxi). Elle ressemble chez ces deux espèces, comme contour, à l'Arca lactea de nos côtes de l'Océan.

Aussitôt que l'animal commence à grandir, cette petite coquille perd ses formes régulières. Une de ses valves (n'importe laquelle) devient adhérente et reste fixée. Alors dans cet état, l'animal se développe comme il peut, s'allonge ou se contourne pour éviter les obstacles et prend toutes les formes possibles pour arriver à son entier développement.

Les $\mathbb{E}$ théries sont les seuls mollusques, je crois, qui ont la faculté de se fixer par l'une ou l'autre valve. Aussi sontelles ou dextres ou sénestres. On reconnaitlla dextrorsité ou la sinistrorsité à la direction du ligament. Chez les Mulleria, la valve droite seule devient et reste adhérente.

Les espèces de ce genre vivent dans les rivières de l'Afrique. Ellles ont été constatées dans tout le bassin du 
Nil, ainsi que dans ceux du Sénégal, de la Gambie et du Niger, aussi bien que dans les eaux des régions d'Angola et de Benguella. Les frères $\mathrm{H}$. et $\mathrm{A}$. Adams (Gener. rec. moll.11, p. 509) en mentionnent une (l'ætheria Stefanensis, Moricand) de l'Amazone. Mais je doute fort de la détermination de cette espèce, qui ne pourrait bien être qu'une Mulleria.

Les $\mathbb{E}$ théries, recueillies dans ces contrées si distantes les unes des autres, ont été regardées soit comme des Caillaudi, soit comme des plumbea. Je pense que sous ces deux appellations on a dû confondre plusieurs formes qui auraient mérité d'être distinguées, et, qu'en voulant trop restreindre le nombre des espèces, on n'ait fait des confusions déplorables. Ce n'est pas une science que celle qui consiste à tout réunir. Ce mode scientifique dénote généralement, chez leurs auteurs, ou absence d'érudition, ou manque de coup d'œil.

Les Æthéries connues sont en petit nombre.

Lamarck en a décrit quatre, les elliptica, trigonula, semilunata et transversa.

Ferussac a créé la Caillaudi et a réuni les quatre espèces de Lamarck en deux, en attribuant à chacune d'elles un nom nouveau. Ainsi, il a appelé Lamarcki les elliptica et trigonula, et Plumbea les semilunata et transversa. Ces appellations ne peuvent être adoptées, parce qu'elles sont contraires aux lois de l'antériorité.

Enfin, Sowerby a fait connailre une forme sous le nom de tubrfera et Michelin une autre sous celui de Carteroni.

Les espèces de ce genre sont si variables et si irrégu- 


\section{$-63-$}

lières que j'ai cherché longtemps les caractères les plus stables d'une bonne classification.

Lamarck a divisé ses quatre Ethéries en deux séries : en espèces pourvues d'une callosité oblongue dans la base de la coquille, et en espèces sans callosité. Sous le nom de callosité, Lamarck comprenait le talon. "Sur un des côtés de la facette lamelleuse de la charnière, dit ce savant auteur (Loc. sup. cit. 1807), on voit une grosse callosité oblongue demi-cylindrique, que le ligament des valves semble envelopper au moins partiellement et qui parait avoir été unie à une callosité semblable sur l'autre valve." "

Sur les quatre espèces lamarckiennes, les elliptica et trigonula possèdent un talon (callosité), les semilunata et transversa n'en ont pas.

Pour moi, je sépare les $\mathbb{E}$ théries en quatre séries, d'après la forme de leurs talons, de leurs ligaments, de leurs contours.

\section{A. Caillaudiana.}

Valve adhérente bien plus longue que la supérieure, pourvue d'un ta!on presque en ligne droite, excessivement allongé, presque d'égale grosseur dans toute sa longueur. - Ligament externe, composé d'une partie fibreuse très résistante contenue dans. un large sillon (dont on voit la trace sur l'étendue entière du talon), et d'une partie ligamenteuse renfermée dans une fente très étroite. 
Test triès feuilleté, oralaire-allongé dépassant médiocrement en largeur le diamètre du talon.

\section{Etheria Caillaudi}

Etheria Caillaudi, Ferussac, Mém. Éthér. in Mém. acad. sc. I. 1823, p. 3399. - Et Caillaud, Voy. à Méroë, II, 1826, p. 222 et IV, 1827, p. 261 et ailas II, 1823, pl. 61, f. $1-3$.

Je ne puis accepter comme représentant cette espèce : $1^{\circ}$ l'Atheria Caillaudi, de Rang et Caillaud (mém. G. Éthér. in Nouv. ann. Mus. III, 1834, pl. 6). - $2^{\circ}$ l'Ætheria Caillaudi de Chenu (Man. Conch. H, 1862, fig. 739). - Je rapporte la première à l'Etheria Letourneuxi; - la seconde à l'elliptica.

La Caillaudi est une espèce de très grande taille, caractérisée par un talon feuilleté d'une excessive longueur (j'en ai vu de plus de 35 cent.) conservant à peu près la même largeur. - Valves irrégulières, toujours d'une forme allongée très oblongue. Intérieur d'une nacre micacée blanchâtre. Extérieur id'un noil' verdâtre, ordinairement excorié, mais lorsqu'il est intact, orné de nombreuses spinules tubuleuses irrégulièrement placées.

Cette espèce a été recueillie pour la première fois, en 1821, en Nubie, dans la province de Robatas, sur les bords du Nil et de ses affluents, où elle est excessivement commune. Depuis elle a été trouvée dans tout le cours du Nil. En Égypte, je la connais des canaux du Fayoum, du Nil 
pres d'Esneh, des bords du lac Mariout où elle a été récoltée morte non loin d'Hagueret en Naouatié, par notre ami le conseiller Letourneux.

Le docteur Chambard l'a découverte dans les couches quaternaires de Ramsès.

Il existe, dans les collections du Muséum de Paris, de magnifiques exemplaires de cette espèce. Il y en a un, entre autres, qui a plus de 40 centimètres de longueur.

\section{B. NiLOTiCiana}

Valve adhérente médiocrement plus grande que l'autre. Talon peu allongé, conıve, presque toujours renversé en arrière ou incliné à gauche ou à droite, à sommet toujours aigu, lorsqu'il n'est pas excorié. Ligament peu allongé, externe. Coquille très irrégulière, affectant toutes les formes, mais, en somme, toujours plus grande, plus large et plus développée que le talon (chez la série précédente le talon, d'une longueur démesurée, est constamment presque aussi large que la coquille). Test feuilleté ou non feuilleté, ne croissant pas comme celui de la Caillaudi, c'est-à-dire en juxtaposant ses couches lamelleuses les unes à la suite des autres dans le sens de la longueur, mais dans le sens de l'épaisseur.

\section{Etheria tubifera}

Etheria tubifera, Sowerby, in Zool. journ.

$$
\text { 1, } 1825, \text { p. } 523 .
$$

Cette espèce, qui jusqu'à ce jour a été confondue avec MAT. 1880. 
la Caillaudi ou la Carteroni, est une forme bien spéciale. Elle possède un talon court, obliquement conique. Sa forme, très irrégulière, a toujours sa plus grande longueur dans le sens transversal.

Valve adhérente plus grande que l'autre. Nacre intérieure d'un vert bleuacé. Surface externe ornée de spinules tubuleuses irrégulièrement espacées. "Valva superiore spinis irregularibus tubulosis conspersa, " dit Sowerby. Long. 110 mill. sur 70 à 75 de hauteur.

Il y a dans les collections du Muséum de Paris, sous le nom de Caillaudi, var. rotundata, plusieurs beaux échantillons de cette espèce, échantillons recueillis dans le Nil, en 1834, par le consul Botta.

\section{Etheria Nilotica}

Cette Ethérie est également une forme particulière et très distincte.

On remarque au Muséum un magnifique groupe de cette espèce, donné par Joannis en 1834, sous le nom de Caillaudi var. elongata. Pour moi, j'en possède deux des bords du lac Mariout, dans la basse Egypte.

\section{ACtheria Elliptica}

Il faut réunir sous ce nom :

$1^{\circ}$ L'elliptica, Lamarck, in Ann. mus. X. 1807, p. 401. pl. 29 et 31. f. 1, et Anim. s. vert. VI, première partie, 1819, p. 100. 
$2^{\circ}$ La trigonula, Lamarck, in. Ann. mus., X, 1807, p. 403, pl. 30 et 31, et Anim. s. vert. VI, première partie 1819, p. 100.

$3^{\circ}$ La semilunata de Sowerby, Genera of shells, première livraison, 1820 (non semilunata de Lamarck, qui est différente).

$4^{\circ}$ La Lamarcki, de Ferussac, in Mém. Acad., sc. 1, 1823, p. 359 (nom nouveau proposé à la place de ceux d'elliptica et de trigonula).

כొ La Caillaudi de Chenu, Man. conch., II, 1862, p. 150. fig. 139 (très-réduite). (Non Caillaudi de Ferussac.)

Je rapporte encore à cette ⿷thérie l'Elliptica de Blainville (Man. malac. p. 543, pl. 70 bis, fig. 2 (très-médiocre).

J'ai été assez heureux, grâce à l'extrême obligeance du professeur de malacologie, d'avoir pu vérifier dans les galeries du Muséum les espèces de Lamarck.

J'ai tenu et examiné les elliptica et trigonula types, qui sont bien une seule et même espèce, comme l'avait affirmé Ferussac. J'ai également vu, dans cette riche collection, l'échantillon représenté dans le Gener shells (1820) par Sowerby, sous le nom de semilunata. Cet échantillon est une elliptica moins avancée en âge. Tous ces individus proviennent du Sénégal. "M. Lajoye, dit Lamarck, dans une note manuscrite, m'a certifié que la trigonula venait du Sénégal. " Ces échantillons ont tous, du reste, un air de parenté qu'on ne saurait méconnaitre. Ils possèdent une nacre interne plombée, commune à toutes les æthéries sénégaliennes.

J'ai rarement vu une plus splendide espèce que l'elliptica de Lamarck; elle mesure 240 millimètres de haut sur 1500 de large. D’après une note manuscrite, celte espèce a 
été achetée par Faujas, chez un marchand de curiosités de la rue Saint-Honoré, près la place du Marché.

La trigonula, qui n'est incontestablement autre chose qu'une elliptica trigone, a 214 millimètres de hauteur sur 133 de large.

Ces æthéries, qui n'ont aucuns rapports avec celles du bassin du Nil, possèdent un test solide, opaque, pesant, non feuilleté, sauf sur un ou deux points; une valve adhérente médiocrement plus grande que la supérieure, et un talon très épais, peu allongé, conico-oblong.

Leur surface supéro-externe est simplement striée et sans spinules. Leur nacre interne est lisse, d'un blane violacé d'une nuance plombée. Chez la trigonula, la nacre est faiblement plombée rougeâtre.

\section{Atheria Senegalica}

Sous cette nouvelle appellation, je rapporte plusieurs formes que je ne puis réunir à l'elliptica ni à aucunes autres, telles que :

$1^{\circ}$ L'ætheria semilunata, de Woodward, Man. conch., pl. 18, f. 7, 18 อ̃ (non semilunata de Lamarck).

$2^{\circ}$ L'xtheria plumbea, de Deshayes, in Dict. d'Hist. nat. de D'Orbigny. - Atlas. moll., pl. 5, f. 3-4 (non plumbea de Ferussac).

$3^{\circ}$ Et plusieurs beaux groupes donnés par Rang au Muséum, et rapportés par cet auteur à la Caillaudi.

Talon court, très conique. - Nacre d'une nuance plombée. Surface extérieure d'une couleur verte. Test très épais vers la région dorsale. 
On remarque sur quelques individus de ces groupes des rudiments de spinules tubuleuses.

L'échantillon figuré par Deshayes sous le nom de plumbea est d'une teinte bronzée avec quelques reflets verdâtres.

Du Sénégal.

\section{C. - Chambardiana}

Valve adhérente pas plus grande que l'autre, talons Egaux sur les deux valves, se recouvrant mutuellement, par conséquent interne, d'une forme conique, contourné en arc de cercle à droite ou à gauche. Ligament interne. Test très feuilleté, ne croissant pas en longueur mais en épaisseur.

Je ne connais qu'une espèce de cette série :

\section{L'Atheria Chambardi}

des couches quartenaires de Ramsès.

\section{D. - Letourneuxiana}

Valve adhérente de même taille que l'autre, quelquefois plus petite. Pas de talon ou talon très exigu, ne dépassant pas les valves. Ligament interne, très court, très robuste, conique. Test opaque, non feuilleté ou à peine feuilleté parfois à l'intérieur. 
Cette sẻrie comprend cinq espèces, les

\section{Etheria Petrettinii}

et

\section{Etheria Letourneuxi}

d'Égypte, dont j'ai donné les signes distinctifs, puis les trois suivantes, des eaux du Sénégal :

\section{Ftheria semilunata}

Etheria semilunata, Lamarck, in Ann. mus. X, 1807, p. 404, pl. 32, f. 1-2, - et Anim. s. vert., VI, $1^{\text {re }}$ partie, 1819, p. 100.

Etheria plumbea (pars), Ferussac, in Mém. acad.sc., I, 1823, p. 359, - et Rang et Caillaud, Mém. Eth., p. 16, 1834.

Je ne puis rapporter à cette espèce la Semilunata représentée par A. et H. Adams, dans leur «Genera of recent mollusca " (pl. 120, f. 3, 1858). Je ne sais ce que peut être cette æthérie.

Je considère la semilunata, malgré l'opinion de Ferussac, comme distincte de la transversa.

Nacre interne assez brillante, d'une nuance violacée légèrement plombée. Surface extérieure d'une couleur bronzée. Talon petit, subconique, ne dépassant pas la valve supérieure. - Hauteur 9ə̃0, largeur 680 millimètres. Du Sénégal. 


\section{Etheria Transversa}

Etheria transversa, Lamarck, in Ann. mus., X, 1807, p. 404, pl. 32, f. 3-4, et Anim.s. vert., IV, $1^{\text {re }}$ partie, 1819, p. 100.

Atheria plumbea (alt. pars), Ferussac, in Mém. acad. sc., 1, 1823, p. 359, - et Rang et Caillaud, Mém. Eth., p. 16, 1834.

Etheria semilunata, Deshayes, Traité Conch. atlas, pl. 41,

f. 1-3, 1853 (non semilunata de Lamarck).

Test toujours plus large que haut (larg. 9ä0, haut. 660 millimètres).

Cette espèce sénégalienne est l'æthérie la plus répandue dans les collections sous le nom de plumbea, à cause de sa nacre d'une nuance très plombée.

\section{Etheria Carteroni}

Etheria Carteroni, Michelin, Mag. zool. moll., 1, 1830, pl. 1, f. 1.

L'échantillon type décrit et figuré (à moitié de sa taille) par Michelin se trouve dans les collections du Muséum. Cet échantillon, d'un vert noirâtre, est orné de 4 rangées de boursouflures, régulièrement divergentes, sur lesquelles se dressent quelques spinules tubuleuses.

Nacre intérieure d'un vert livide. Test solide, pesant, non feuilleté, peu épais, d'une forme obliquement subovalaire. Talon petit, ne dépassant pas les valves. 
La Carteroni ne peut ètre confondue avec la tubiferct de Sowerby.

En résumé, d'après mes connaissances actuelles, il existerait en Égypte six espèces d'æthéries :

$1^{\circ} \mathrm{La}$ Caillaudi, caractérisée par un talon (non conique) d'une excessive longueur.

$2^{\circ}$ La tubifera, à talon court et conique.

Ces deux espèces, d'une teinte externe verdâtre, sont tubifères.

$3^{\circ}$ La nilotica, non tubifère, d'une couleur de feuilles mortes, à valves inégales et à talon très largement conicopyramidal.

$4^{\circ}$ La Chambardi (espèce quartenaire, probablement éteinte), à talons égaux, coniques, toujours recouverts par des valves de même taille.

Enfin $5^{\circ}$ la Petrettinii.

Et $6^{\circ}$ la Letourneuxi, qui, toutes deux, ne possèdent pas de talons. 


\title{
DES CRIPTION
}

\author{
DU NOUVEAU GENRE
}

\section{GOIエ上TOPT上RUM}

Les espèces pour lesquelles j'établis ce nouveau nom gé-

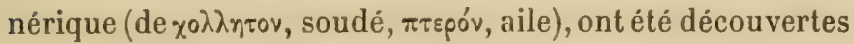
en septembre 1879, dans le Danube, par notre ami le conseiller Letourneux.

Ces espèces, d'apparence anodontoïde, offrent les caractères suivants :

1. Charnière arquée, très courte, sans dents, mais présentant néanmoins, à l'endroit où se développe, chez les unios, la lamelle latérale, un épaississement souvent considérable, d'un relief prononcé, ressemblant à une denticulation lamelliforme. Cette lame opaque, d'un blanc nacré, ordinairement plane ou presque plane en dessus, offre parfois (notamment chez le præclarum) une ligne saillante, sensible surtout vers la région des crochets qui, sur la valve gauche, est reçue dans un léger sillon. 
2. Ligaments internes, dont l'un fliforme antérieur, s'étendant des crochets à l'angle antéro-dorsal, et l'autre fibreux, gros et court, terminé à son extrémité par une vaste lunule, presque aussi longue que la lamelle latérale.

$3^{\circ}$ Bord supérieur soudé d'un bout à l'autre et projetant en arrière des sommets et au-dessus du ligament, qui est complètement recouvert, un aileron mince el très aplati, bien que les deux valves soudées et plaquées l'une sur l'autre forment une double épaisseur. Cet aileron est förmé par un prolongement exagéré de la crête dorsale. On remarque encore quelquefois (comme chez le Letourneuxi) un autre petit aileron à la partie antérosupérieure. Mais celui-ci est plus exigu et moins élevé.

A ces caractères, qui ont leur importance, j'ajouterai que les colletoptères minces, délicats, ornés de striations d'une extrême ténuité, sont généralement de taille médiocre, d'une forme ovalaire dilatée et faiblement ventrue. Leurs sommets, légèrement ridés, sont terminés par des crochets très aigus; enfin, leur coquille est un peu bâillante en dessous de l'angle postéro-dorsal et vers la région inféro-palléale.

Quant aux impressions, peu sensibles du reste, elles me paraissent analogues à celles que l'on remarque chez les Naïades.

Malgré les liens de parenté qu'ils paraissent avoir avec les Anodontes, les Colletoptères ne peuvent être réunis, à mon avis, aux espèces de ce genre.

Je sais bien que toutes les anodontes, dans le jeune âge, sont soudées à leur partie supérieure. Je sais bien encore que, chez plusieurs espèces de certains groupes, le test continue à recouvrir, dans l'àge adulte, le ligament pos- 
térieur. Le genre Symphynota a été établi pour ces espèces. Mais, à mon sens, ce qui différencie le plus les Colletoptères des Anodontes, c'est leur charnière courte et arquée, c'est leur lamelle latérale, ce sont enfin leurs ligaments internes; et, lorsqu'à ces caractères viennent se joindre celui de la partie supérieure, soudée d'un bout à l'autre, celui enfin des ailerons, on est forcé de reconnaitre que ces espèces possèdent des signes génériques différents de ceux des anodontes.

En dehors des anodontes, le genre qui me parait offrir le plus d'analogie avec celui-ci est le genre Dipsas de Leach (Zool. misc., 1, 1814, p. 119).

Cette coupe générique a été établie pour une singulière coquille, à test plissé, à valves soudées, biailées et possédant une charnière pourvue dans toute sa longueur d'une dent lamelliforme saillante. Ce dernier caractère sépare les Dipsas des Colletopterum.

Un fait particulier, sur lequel j'appelle l'attention. L'espèce type (Dipsas plicatus) de Leach est une coquille du British museum qui, jadis, au temps de Leach, était signalée de la Bohême. "A Bohemian river horse-mussel. " Or, on ne connait actuellement des Dipsas que du centre de l'Asie. Ce genre existerait-il en Bohême? Pourquoi non? puisque les Colletoptères, qui sont voisins des Dipsas, vivent dans le Danube.

On ne peut non plus réunir les espèces de notre nouveau genre aux Cristaria de Schumacher (Ess. nouv, syst. vers test., p. 106, pl. XX, f. 1, 1817), que l'on a confondu, je ne sais pour quel motif, avec les Dipsas, bien qu'ils soient très dissemblables de ceux-ci.

Les Cristaria, en effet, sont caractérisés par un aileron postérieur; par une charnière pourvue d'une fossette car- 
dinale linéaire et rectiligne et d'une latérale forte et bifide; enfin, par un fort ligament externe. On voit par ces caractères, que ce genre ne peut rentrer ni dans les Dipsas, ni dans les Colletopterum. Il devrait plutôt passer en synonymie des Barbala d'Humphrey (Mus. calonn., p. 59, 1797).

Les Colletoptères se divisent en deux séries : $1^{\circ}$ en espèces biailées, c'est-à-dire ornées de deux ailerons, l'un postérieur et l'autre antérieur; $2^{\circ}$ en espèces uniailées possédant seulement un aileron postérieur.

Le Colletopterum Letourneuxi appartient à la $\mathbf{1}^{\text {re }}$ série. Les præclarum, eximium et Tanousi, à la seconde.

\section{Colletopterum Istourneaxi}

Long. $\max \ldots \ldots \ldots \ldots \ldots \ldots \ldots$ 5ร mill.

Haut. $\max . . . \ldots \ldots \ldots \ldots . . . . .42-$

Épaiss. max. (à 15 en dessous et en arrière des crochets, $29 \mathrm{du}$ rostre, 28 du bord antérieur, $191 / 2$ de l'angle postéro-dorsal, 25 de la base de la perpendiculaire)...$\ldots \ldots \ldots$.

Longueur de la crête ligamenlo-dorsale, du sommet à l'angle postéro-dorsal. Distance de cet angle au rostre..... 31

Corde apico-rostrale............ 43

Hauteur de la perpendiculaire...... 38 -

Distance de cette perpend. au bord antérieur................

- du même point au rostre postérieur................. 31 - 
Distance enfin, de la base /de la per-

pend., à l'angle postéro-dorsal. 42 mill.

Coq. de forme oblongue, d'après une direction obliquement descendante de gauche à droite, caractérisée par un bord supérieur sur lequel on remarque : $1^{\circ}$ à la partie antérieure une petite région ailée, très aplatie, peu élevée, formant un angle de $130^{\circ}$ avec le bord antérieur; $2^{\circ}$ à la partie postérieure, une longue crête aliforme, élevée, très comprimée, extérieurement plate et formant avec le bord postéro-dorsal, qui est brusquement descendant, un angle de $110^{\circ}$.

Valves très comprimées, médiocrement convexes (convexité maximum en arrière et assez rapprochée des sommets).

Bord supérieur sans direction accusée, par suite de ses deux ailerons à contours légèrement accidentés. Partie antérieure arrondie, relativement petite dans'le sens de la hauteur. Bord inférieur convexe descendant. Partie postérieure largement dilatée en hauteur, peu allongée, pourvue d'un rostre très obtus, arrondi. Entre ce rostre et l'angle postéro-dorsal, le contour est sensiblement concave.

Sommets comprimés, écrasés, ridés (rides ondulées), à crochets aigus. Crête dorsale aplatie, très dilatée en hauteur et en longueur en forme d'aileron. Arête dorsale assez accentuee, un tant soit peu convexe.

Stries d'accroissement délicates, très émoussées, sauf sur la région postéro-dorsale où elles sont bien marquées.

Épiderme très brillant, d'un jaune clair, passant à une nuance légèrement rougeâtre vers les sommets et verdâtre sur la région postéro-dorsale, avec quelques radiations d'une teinte plus foncée.

Nacre intérieure irisée, blanchâtre. 
Ligament antérieur filiforme, se prolongeant assez loin en arrière des crochets sous l'autre ligament.

Ligament postérieur, entièrement recouvert, court, se terminant à 10 mill. en avant de l'angle postéro-dorsal par une longue lunule qui s'étend entre les deux parois de l'aileron.

Charnière arquée, courte. Partie cardinale tellement mince qu'elle est à peine perceptible. Partie latérale d'un blanc nacré, assez épaisse, s'écartant à son extrémité (par suite de la courbure de la charnière) du ligament postérieur. Une petite lamelle filiforme vers la partie antérieure; lamelle reçue dans une gouttière sur l'autre valve.

Impressions peu accentuées; l'antérieure en éventail (la pointe en haut); la postérieure irrégulière.

Cette belle espèce, que je me fais un plaisir de dédier à notre ami le conseiller Letourneux, a été trouvée par cet infatigable et heureux explorateur, dans le Danube près de Belgrade (Serbie).

\section{Colletopterum præelarum}

Long. max................ 73 mill.

Haut. $\max . . . \ldots \ldots \ldots \ldots \ldots . \ldots \ldots 61 / 2$

Epaiss. max. (à 21 au-dessous et en arrière des crochets, $\mathbf{4 1}$ du rostre, 31 1/2 du bord antér, 27 de l'angle postéro-dorsal, 32 de la base de la perpend..................

Long. de la crête ligamento-dorsale, du sommet à l'angle postéro-dorsal. Dist. de cet angle au rostre........ 47 - 
Corde apico-rostrale........... 58 1/2 mill.

Haut. de la perpendiculaire....... 53 -

Dist. de cette perpend. au bord antér. $29-$

- du même point au rostre postér.. 44 -

- enfin de la base de la perpend. à l'angle postéro-dorsal......... 57 -

Coq. de forme largement subovale-descendante, anguleuse seulement à son extrémité postéro-dorsale, caractérisée à sa partie postérieure par une région rostrale très développée, très large, parfaitement arrondie, et, par des valves peu ventrues, régulièrement convexes.

Bord supérieur presque rectiligne. Partie antérieure sphérique. Bord inférieur faiblement convexe-descendant. Partie postérieure très largement dilatée avec une région rostrale bien ronde, sans partie anguleuse appréciable. Crête dorsale dilatée, comprimée, coupée brusquement sous un angle de $115^{\circ}$. Arête dorsale à peine sensible.

Sommets assez distants du bord antérieur (29 mill.), comprimés, non proéminents, couverts de rides légèrement ondulées.

Stries d'accroissement, délicates, très émoussées, feuilletées seulement sur la région postéro-dorsale, et représentant exactement la forme des contours.

Epiderme très brillant (sauf sur la partie feuilletée), d'une teinte indécise, variant du jaune-verdâtre vers la région rostrale, à un ton jaunacé vers l'antérieure, puis à une nuance jaune-cendrée sur la partie médiane pour passer, vers les sommets, à une couleur ochracée légèrement rougeâtre, et ornée, en outre, de légères radiations verdâtres, sensibles, seulement, vers la région postérieure et près des contours. 
Nacre intérieure bien irisée, d'une nuance blanche bleuacée, avec des tons faiblement orangés vers sa charnière.

Ligament antérieur filiforme, membranoïde, se prolongeant de 3 mill. en arrière des crochets sous l'autre ligament.

Ligament postérieur, entièrement recouvert, très courı, robuste, se terminant à 8 mill. en avant de l'angle postérodorsal par une large lunule à reflet métallique.

Charnière très arquée, relativement très courte, partie cardinale filiforme, partie latérale épaisse, robuste, bien en relief, d'une nacre blanche, s'écartant de 5 mill. à son extrémité postérieure (par suite de la courbure de la charnière) du ligament postéro-interne. Sur cette lame laté. rale, on remarque, notamment vers les crochets, c'est-àdire à sa partie antérieure, une lamellule saillante, reçue sur l'autre valve dans une gouttière allongée et assez accentuée.

Impression peu prononcée; l'antérieure irrégulière, biou-tripartie; la postérieure spathuliforme.

Le præclarum vit en Bulgarie, dans le Lom près de Rustchuk, sur la rive droite du Danube (Letourneux).

\section{Colletopterum eximium.}

Long. $\max . . . \ldots \ldots \ldots \ldots . . . .67$ mill.

Haut. $\max . . \ldots \ldots \ldots \ldots \ldots \ldots, 46 \quad 1 / 2$

Epaiss. max. (à 25 en dessous et en arrière des crochets, -36 du rostre, -30 du bord antér., -28 de l'angle postéro-dorsal, $\mathbf{- 1 8}$ de la base de la perpend.)............. 23 - 
Long. de la crête ligam. dors., du sommet à l'angle postéro-dorsal..... 24 mill.

Dist. de cet angle au rostre........ 32 -

Corde apico-rostrale............ 50 $1 / 2$

Haut. de la perpendiculaire....... 41 1/2

Dist. de cette perpend. au bord antérieur................ 23 -

- du même pointau rostre postérieur 43 -

- enfin, de la base de la perpend. à l'angle postéro-dorsal........ 47 1/2

Coq. ovalaire-oblongue, légèrement rostrée à sa partie postërieure (rostre arrondi, peu prononcé, très court, un tant soit peu remontant et regardant horizontalement à droite).

Valves relativement plus ventrues que celles de l'espèce précédente, avec le maximum de la convexité plus inférieur.

Bord supérieur bien rectiligne. Partie antérieure arrondie, anguleuse à sa jonction avec le bord dorsal. Bord inférieur convexe-arrondi (plus forte convexité vers les 2/3 postérieurs). Partie postérieure près de deux fois plus longue que l'antérieure, rostrée (rostre bien prononcé). Crête dorsale, dilatée, comprimée, coupée brusquement sous un angle de $130^{\circ}$. Arête dorsale assez accentuée, légèrement subarquée.

Sommets comprimés, comme écrasés, ornés de rides ondulées. Crochets très aigus.

Stries d'accroissement excessivement tenues, régulières, émoussées, feuilletées sur la région postéro-dorsale.

Epiderme assez brillant, d'une teinte verte-jaunacée, ochracée sur les sommets, et, obscurément radiatulé de MAI 1880. 
bandes plus foncées, visibles seulement à la partie postérieure.

Nacre intérieure irisée blanche, tantôt bleuacée, tantôt orangée.

Ligament antérieur interne, filiforme, se confondant à son extrémité avec le ligament postérieur. Celui-ci gros, court, robuste, complètement recouvert, se termine à 8 mill. de l'angle postéro-dorsal, sous la forme d'une vaste lunule brunâtre.

Charnière un peu moins arquée que celle du præclarum. Région cardinale nulle, filiforme. Région latérale forte, épaisse, encrassée d'une nacre bien blanche, présentant (sur l'une et l'autre valve) une lamelle aiguë, bordée par un sillon.

Impressions: antérieure irrégulièrement oblongue ou arrondie; postérieure subtriangulaire.

Cette espèce a été recueillie sur la rive gauche du Danube à Giurgervo en Valachie et dans le Lom, près de Rustchuk, en Bulgarie (Letourneux).

L'eximium se distingue du proclarum: $1^{\circ}$ par sa taille moindre; par sa forme postérieurement plus longue, assez nettement rostrée, tandis que le praclarum présente une partie postérieure largement dilatée-arrondie sans rostre sensible. Chez l'eximium, le rostre regarde horizontalement à droite, tout en étant un tant soit peu relevé; chez le proclarum, la partie postéro-rostrale paraît descendante par suite de sa grande dilatation inférieure;

$2^{\circ}$ Par ses sommets plus antérieurs et plus comprimés que ceux du præclarum;

$3^{\circ}$ Par sa convexité maximum plus inférieure, ce qui 
fait paraître cette espèce plus ventrue, bien qu'elle soit plus petite;

$4^{\circ}$ Par son angle postéro-dorsal plus obtus $\left(130^{\circ}\right.$ au lieu de 115 chez le præclarum);

$5^{\circ}$ Par son arête dorsale assez accentuée, légèrement arquée. Elle est à peine sensible chez le præclarum;

$6^{\circ}$ Par son ligament antérieur, filiforme, qui, à son extrémité,se réunit au ligament postérieur.Chezle procclarum, il se prolonge de 3 mill. en arrière des crochets, sous le ligament postérieur, sans se confondre avec lui;

$7^{\circ}$ Par sa charnière moins arquée, etc.

\section{Colletopterum Tanousi.}

Long. $\max . . . \ldots \ldots \ldots \ldots \ldots . . .58$ mill.

Haut. $\max . . . . \ldots \ldots \ldots \ldots \ldots . . . .43$ -

Epaiss. max. (à 22 en dessous et en arrière des crochets, -27 du rostre,

- 29 du bord antér, -23 de l'angle postéro-dorsal, - 19 de la base de la perpendiculalre)........... 19 -

Long. de la crête ligam. dors., du sommet à l'angle postéro-dorsal..... 22 -

Dist. de cet angle au rostre......... 30 -

Corde apico-rostrale............ 40ั -

Hauteur de la perpendiculaire...... 38 -

Dist. de cette perpendiculaire au bord antérieur.............. 20 -

- du même point au rostre postérieur................. 38 - 
Dist. enfin, de la base de la perpend.

à l'angle postéro-dorsal..... 43 mill.

Coq. de forme exactement oblongue, d'après une direction descendante de gauche à droite, sans angle ni rostre appréciable, sauf à l'endroit de la crête dorsale où s'élève un aileron aplati, très comprimé et fortement dilaté.

Valves régulièrement convexes, avec le maximum de la convexité à 22 mill, au-dessous et en arrière et à 27 mill. seulement de l'extrémité rostrale. Chez les autres espèces, la convexité est plus supérieure et moins postérieure.

Bord supérieur rectiligne, sauf sur la crête dorsale, où celle-ci se relève en aileron. Partie antérieure bien arrondie. Bord inférieur convexe. Partie postérieure, à contours arrondis, près du double plus longue que l'antérieure.

Sommets comprimés, comme écrasés, ridés, à crochets très pointus.

Stries d'accroissement très délicates, feuilletées seulement vers la région postéro-dorsale, reproduisant les contours exacts des valves.

Épiderme brillant, presque lisse, d'une leinte verdâtre, passant vers les sommets à une nuance jaunacée un peu rougeàtre, et ornée d'une multitude de radiations verdâtres peu prononcées.

Nacre intérieure éclatante, irisée, blanche avec des tons orangés.

Ligament antérieur filiforme, se prolongeant de $\mathbf{5}$ mill. en arrière des crochets sous l'autre ligament. Celui-ci, assez allongé, peu épais, est complètement recouvert par l'expansion ailee qui le dépasse de 3 mill.

Charnière médiocrement arquée, relativement assez 
longue. Région cardinale filiforme, avec une gouttière médiane, ce qui fait paraitre cette partie comme sillonnée par de petites lamelles. Région latérale assez large, avec une ligne lamelliforme sur toute sa longueur.

Impressions : antérieure triangulaire; postérieure spathuliforme.

Le Tanousi, que je dédie au Syrien Tanous Farez, a été recueilli dans le Danube, près de Belgrade, en Serbie (Letourneux).

Chez cette espèce, très distincte des précédentes, on remarque, à l'extrémité antéro-dorsale, à l'endroit où le bord supérieur fait sa jonction avec le contour antérieur, une petite partie comprimée, même aplatie, qui rassemble à un commencement d'aileron. 
DES

\title{
AINODONTES
}

\author{
DES RÉGIONS OCCIDENTALES DE L ASIK
}

On connait fort peu d'Anodontes de ces régions asiatiques qui dépendent du système européen. Quelques-unes ont été signalées dans le Turkestan, la Mésopotamie, l'Anatolie et les pays transcaucasiques. Je ne sache point que l'on en ait jamais trouvé en Perse, en Arabie, en Syrie et en Palestine.

Voici le résumé des connaissances sur ces espèces.

1837. Honenacker (Enumeratio anim. quæ in prov. transc. Karabach, Schirwan et 'Talysck; etc., in Bull. soc. Moscou, p. 147), constate la présence de l'Anodonta intermedia de Lamarck dans les ruisseaux de la province de Talysck. J'ai de grands doules sur la bonne détermination de cette espèce. 
1857. Bourgdignat (Deux. suppl. aux bivalves de l'emp. ottoman, in Amén. Malac., II, p. 34, pl. 1, f. 1-2), décrit l'Anodonta Vescoiana des environs de Koniek (ancienne Iconium), dans la partie presque centrale de l'Anatolie. Cette Anodonte (forme caractéristique des eaux de cette région) descend l'Euphrate jusqu'à Bagdad, où elle parait abondante.

1863. Mousson (Coq. terr. fluv. rec. dans l'Orient par le $D^{\mathrm{r}} \mathrm{Al}$. Schlœfli, II, p. 94) appelle l'attention sur des débris d'Anodontes indéterminables, rejetés par le Phase (Rheon) près de Poti, sur la Mer Noire.

1874. Martens (Ueber vorderasiatische Conchylien, p. 69) cite l'Anodonta intermedia signalée par Hohenacker dans la province de Talysck, et, mentionne mon Anodonta Vescoiana à Bagdad.

1874. Martens (Reise in Turkestan von Al. Fedtschenko. - Mollusca, p. 33) indique l'Anodonta piscinalis de Nilsson.

1874. Mousson (Coq. terr. fluv, rec. par le DrAl. Schlofli dans l'Orient, III, p. 46 et 47 ) décrit, sous le nom de mesopotamica, une forme plus grande (long. 124, hauteur 506, épaiss. 65 mill.) de la Vescoiana des environs de Bagdad. Cette forme que l'auteur m'avait envoyée dans le temps, sous l'appellation de Vescoiana Var. turgidula de Menke (qu'il m'est impossible de distinguer du type), est représentée sur la planche XIII (fig. 1-3), qui accompagne ce travail.

A cette espèce, l'auteur en propose une autre, égale 
ment de Bagdad, l'Anodonta Schlœflii qu'il décrit en ces termes:

"Concha magna, maxime inflata, obtuse subtriangularis, subæquilateralis, minus fragilis, striata, fusco-lutea, intus cœrulescente-alba. Umbones inflati, producti, antice deflexi. Margo superus brevis, subarcuatus, ligamento brevi; anterior breviter incurvatus ; posterior parum productus, subangulatus; inferus bene curvatus, long. 10马, alt. 77, crass. 60 millim. - Cette forme n'est peut-être qu'une variété ou même qu'une modification individuelle de la Vescoiana. Mais ne pouvant décider la question sur les individus à ma disposition, je préfère appuyer sur les différences, savoir : un contour moins allongé, subtriangulaire; un test un peu plus solide; un bord supérieur plus court, courbé et non droit; un bord palléal antérieur moins largement arrondi ; un bord inférieur plus régulièrement courbé; des crochets plus renflés. "

1876. Mousson (Coq. recueillies par le $\mathrm{D}^{\mathrm{r}}$ Sievers dans la contrée transcaucasique. II, p. 25 (tirage à part) cite l'anodonta anatina de Linnœus, dans le Gergerep, un des affluents du Kura.

1878. Scunkider (Kaukasische Conchylien, p. 33) constate la présence de l'A nodonta ponderosa de G. Pfeiffer, à l'embouchure des rivières dans la mer Caspienne, près de Lenkoran.

En somme, on ne voit indiquer, dans les contrées occidento-asiatiques, que six espèces, dont quatre les: INTERmedia, Piscinalis, Anatina et Ponderosa, seraient sembla$\therefore$ 
bles à celles de l'Europe (ce dont je doute fort); et deux vraiment asiatiques, les Vescoiana et Schlokfli.

Le genre Anodonte est donc bien faiblement représenté dans ces régions.

A ces espèces, j'ai à ajouter trois anodontes nouvelles. Ces acéphales, très caractérisés, proviennent du lac Apollonia (anciennement Apolloniates) à 44 kil. au S. 0. de Brousse et du ruisseau de Kadi-Keui, dans l'Anatolie.

\section{Anodonta taurica.}

Pl. XIV, f. 1-4

Long. $\max . \ldots \ldots \ldots \ldots \ldots . . . . . .113$ mill.

Haut. $\max . . . . . . . . . . . . . . .674-$

Epaiss. max (à 42 en dessous et en arrière des, sommets, à 5 s̆ du rostre, à 57 du bord antérieur, à 32 de l'angle postéro-dorsal, à 42 de la base de la perpendiculaire)......

Long. de la crête ligam. dors., du sommet à l'angle postéro-dorsal. . $\quad 52$ -

Dist. de cet angle au rostre....... 52 -

Corde apico-rostrale............ 95 -

Hauteur de la perpendiculaire..... $69 \quad$

Dist. de cette perpendiculaire au bord antérieur............... 
Dist. du même point au rostre postérieur...................

79 mill.

- enfin, de la base de la perpend. à l'angle postéro-dorsal.....

$73-$

Coq. "exactement oblongue (sans parties anguleuses appréciables), d'après une direction un peu descendante de gauche à droite, à valves solides, assez épaisses, opaques, régulièrement ventrues-convexes. - Convexité assez médiane.

Bord supérieur arqué. Partie antérieure bien sphérique. Bord inférieur convexe. Partie postérieure oblongueallongée, à contour arrondi, sans rostre bien défini.

Sommets déprimés, comme écrasés, non proéminents, ne déformant par aucune saillie la convexité régulière du test (prise dans le sens de la hauteur), ornés de rides obsolètes concentriques et légèrement ondulées ou plutôt tremblotées. Crochets très aigus, non saillants. Crête dorsale peu développée, assez convexe, régulièrement arquée dans le sens de la longueur. Arête dorsale peu sensible, sans relief sur la convexité des valves.

Stries d'accroissement saillantes, çà et là rugueuses et exfoliées (surtout vers les contours), ne représentant pas toutes le contour exact. Ainsi, à l'état jeune le test (d'après les stries) a une partie antérieure très exiguë, un bord inférieur bien convexe et une région postérieure bien arrondie et moins allongée. A cet âge, la taurica est subovoïde-arrondie. En grandissant, leș stries, en prenant plus de développement sur certaines parties et moins sur d'autres, ramènent cette coquille à une forme exactement oblongue.

Epiderme assez brillant (souvent excoriê), d'un ton in- 
décis jaunacé-verdâtre, tirant vers les contours sur le brun, sur la partie convexe des valves sur le cendré. Nacre interne assez terne, peu irisée, d'un ton blancgrisâtre d'une nuance jaunacée-livide.

Deux ligaments, comme, du reste, chez toutes les anodontes.

Ligament antérieur, interne (long. 44-4̌̃ mill.) commençant à l'extrémité antéro-dorsale, occupant toute l'épaisseur de la charnière et se poursuivant à 22 mill. en arrière des crochets sous l'autre ligament sans se confondre avec lui.

Ligament postérieur, externe (symphynoté dans le jeune âge), fort robuste (long. 46 mill.), d'une teinte marron, terminé par une médiocre lunule de 6 mill.

Charnière sans denticulation, servant de surface apophysiale au ligament antéro-interne sur une longueur de 44 à 45 mill.

Impressions très irrégulières et si changeantes que je crois inutile de les caractériser.

Je ne puis rapprocher cette espèce du lac Apollonia, d'aucunes autres d'Europe.

\section{Anodonta Apollonica.}

Pl. XV, f. 1-3.

Long. max.............. $1221 / 2$ mill.

Haut. $\max . . . . . . . . . . .6771 / 2 \quad$

Epaiss. max. (à 32 en dessous et en arrière des sommets, à 64 du rostre, à 62 du bord anté- 
rieur, à 30 de l'angle postéro . dorsal, à 49 de la base de la perpendiculaire)...........

46 mill.

Long. de la crête ligam. dors., du sommet à l'angle postérodorsal.................

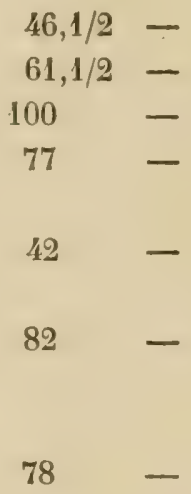

Coq. ovoïde-oblongue, à région antérieure plus largement développée (dans le sens de la hauteur), plus régulièrement sphérique que la région postérieure rétrécie en hauteur et allant en s'atténuant jusqu'à son extrémité rostrale. La hauteur maximum coïncide presque avec la perpendiculaire, puisqu'il n'y a qu'un demi-millimètre de différence.

Valves épaisses, pesantes, solides, ventrues-convexes (ép. 46 mill.). Convexité maximum assez rapprochée de l'angle postéro-dorsal, presque à distance égale entre le rostre et le bord antérieur.

Bord supérieur arqué. Partie antérieure exactement ronde, largement développée. Bord inférieur, d'abord convexe, puis presque rectiligne vers son milieu, enfin convexe-remonlant vers le rostre. Partie postérieure près de deux 
fois plus longue que l'antérieure, très réduite dans le sens de la hauteur (par suite du bord postéro-dorsal très descendant), et terminé par un rostre arrondi.

Sommets convexes, très obtus, à peine proéminents, ornés de rides concentriques très émoussées. Crochets aigus. Grête dorsale réduite, non saillante, comme tronquée, offrant une direction descendante régulière des sommets au rostre. Arête dorsale peu accentuée.

Stries d'accroissement peu accentuées sur la convexité, ressemblant, au contraire, à de fortes côtes vers les contours.

Épiderme d'un jaune paille, brunâtre vers les bords, avec quelques zones concentriques d'un ton plus foncé vers la partie moyenne des valves.

Nacre intérieure d'un blanc éclatant, irisée, très nettement chagrinée dans toute la région concave des valves et très élégamment frangée sur toute l'étendue de l'impression palléale; à chaque frange, correspond une costulation qui s'étend jusqu'à la région chagrinée. Ces franges et ces costulations existent sur les échantillons jeunes. Le chagrinage seul fait défaut.

Ligament antérieur, interne (long. 47 mill.) s'insérant sur la surface entière de la charnière et se poursuivant à 17 mill. en arrière des crochets, sous l'autre ligament; celui-ci externe (long. 44 mill.), fort, robuste, noir, se termine par une lunule de 8 mill.

Charnière sans denticulation, large et épaisse. Impressions très prononcées; l'anterieure composée d'une grande empreinte semi-lunaire, avec deux autres petites ovoïdes ou subquadrangulaires; la postérieure large, semi-arrondie avec une pointe au sommet; la cardinale (située sous les crochets) multiple et profonde; la palléale frangée. 
Je ne puis comparer l'A pollonica qu'avec la gravida du lac Copaïs en Grèce (Journ. conch., p. 142. 1879).

On distinguera l'apollonica de cette espèce: à son test plus épais, plus pesant, moins allongé, relativement plus haut, puisqu'il atteint 77 mill. $1 / 2$ de hauteur sur 122 mill. 1/2 de longueur; tandis que la gravida n'a que 80 de haut sur une longueur de $\mathbf{1 4 0}$ mill.;

A ses sommets plus médiaux; à sa région antérieure plus largement développée, puisque le rayon maximum de la perpendiculaire au bord antérieur est de 42, lorsque celui de la gravida n'est que de 38 mill.;

A sa région postérieure moins allongée, à rostre plus court, arrondi, non troncatulé. Chez la gravida, le rostre a une tendance plus ascendante; le bord postéro-dorsal a une inclinaison moins régulière; l'arête dorsale est mieux marquée, etc.

A sa coloration différente; surtout à sa nacre bien blanche, chagrinée, costulée et frangée. Chez la gravida l'épiderme, d'un brun marron, est parfois olivâtre. Sa nacre est d'une belle teinte bleuâtre passant à une nuance rougeâtre violacée vers la région des sommets;

A son ligament antéro-interne bien plus volumineux et plus allongé. Celui de la gravida n'a que 30 à 32 au lieu de 47 mill., etc.

J'ajouterai encore que chez la gravida, le bord inférieur offre, au niveau de la perpendiculaire des sommets, une convexité très accentuée, que l'on ne remarque pas à un point aussi prononcé chez l'A pollonica. C'est cette convexité que l'auteur de cette espèce, d'après un système nouveau, peu digne d'éloges, qualifie par ces mots "infra concava." 
J'ai donné la représentation exacte des Anodonta Vescoiana, VAR. Mesopotamica de Bagdad, Taurica et Apollonica, sur les planches XIII, XIV et XV. Ces planches, ainsi qu'un grand nombre d'autres dont je compte orner mon travail, ont été exécutées, voilà près de vingt ans, par le célèbre artiste Levasseur.

Ces planches inédites devaient faire partie d'un ouvrage important, que j'ai laissé de côté.

J'ai cru devoir donner cet avertissement, parce qu'à l'époque où je les ai fait exécuter, les acéphales ont été dessinés dans une position qui n'est pas celle d'après laquelle je décris actuellement les bivalves.

\section{Anodonta Gallandi.}

Long. $\max . \ldots \ldots \ldots \ldots \ldots \ldots . . . .122$ mill.

Haut. $\max . . . \ldots \ldots \ldots \ldots . . .6 .65$

Epaiss. max. (à 24 au-dessous et en arrière des crochets, - à 71 1/2 du rostre, - à ร̌s du bord antérieur, - à 29 de l'angle postérodorsal, - $\mathbf{a} 46$ de la base de la perpendiculaire) ...............

Long. de la crête ligam. dors., du sommet à l'angle postéro-dorsal.

Dist. de cet angle au rostre....... 5 57 -

Corde apico-rostrale........... 9 9 -

Hauteur de la perpendiculaire..... $58 \quad-$

Dist. de cette perp. au bord antérieur. $36 \quad$

- du même point au rostre postérieur................ $86 \quad-$ 
Dist. enfin, de la base de la perpend.
à l'angle postéro-dorsal.....
70 mill.

Coq. allongée, de forme très oblongue dans une direction légèrement descendante, caractérisée par une convexité maximum très rapprochée du bord supérieur, et, par son épaisseur, allant, à partir de ce point, en arrière, et, notamment en avant, en s'alténuant d'une façon plane, tectiforme, sans renflement, ce qui donne à cette espèce un cachet tout particulier.

Valves peu épaisses, bien que très résistantes, très bâillantes: $1^{\circ}$ vers la partie médiane du bord palléal; $2^{\circ}$ audessous de l'angle postéro-dorsal.

Bord supérieur faiblement arqué, presque rectiligne. Partie antérieure très-exiguë, comprimée, à contour arrondi. Bord inférieur rectiligne descendant, se relevant vers le rostre en une courbe régulière. Partie postérieure, près du double plus allongée que l'antérieure, terminée par un rostre troncatulé, regardant un peu en bas et offrant au-dessus, jusqu'à l'angle postéro-dorsal, un contour un tant soit peu concave.

Sommets convexes, obtus, proéminents, ornés de petites lignes tremblotées. Crochets aigus.

Arête dorsale très accentuée, offrant deux lignes divergentes dont les extrémités aboutissent aux deux angles du rostre troncatulé. Crête dorsale peu élevée, assez comprimée.

Epiderme d'un ton sale cendré-noirâtre, avec des nuances incertaines d'un vert jaunacé et quelques radiations verles sur la partie postérieure.

Nacre intérieure d'un blanc bleuacé très irisé.

Ligament antérieur, interne, très feuilleté, très épais, 
occupant toute l'épaisseur de la charnière et se poursuivant sous le ligament postérieur à 10 mill. au delà des crochets. Ligament postérieur, externe, très allongé ( 46 mill.), mince, présentant à son extrémité une lunule oblongue (long. 11 mill.). A l'état jeune, ce ligament est recouvert par le test.

Charnière sans denticulation. Région cardinale nulle, remplacée par le ligament antéro-interne. Région latérale étroite, convexe, très allongée, d'une nacre blanche. Impressions bien accentuées, très irrégulières, le plus souvent spathuliformes.

Cette Anodonte, que je dédie à M. Jules Galland, ingénieur des Ponts et Chaussées, au service du gouvernement Ottoman, vit dans un grand nombre de cours d'eau de la côte anatolique. Elle a été notamment recueillie dans le ruisseau de Mada, à Kadi-Keui.

Gette espèce est édule. Elle se vend sur les marchés de Constantinople. C'est de ces marchés que proviennent mes plus beaux échantillons.

La Gallandi, qui n'a aucun rapport avec les Anodontes précédentes, est un Acéphale servant de type de groupe à toute une série d'espèces, que je décrirai au chapitre suivant, espèces spéciales aux eaux de la Crimće et de la Roumélie. 


\section{A NODONTES}

D'EUROPE

Les Anodontes d'Europe sont, parmi les Acéphales, les espèces les plus difficiles à bien déterminer, et cela pour plusieurs raisons.

D'abord, parce que les premières espèces ont été fort imparfaitement caractérisées.

Ensuite, parce que les auteurs, sans chercher à se rendre compte de ce que pouvaient être les formes primitives, se sont créés, pour le besoin de leurs causes, des types factices, qu'ils ont présentés comme les espèces premières.

Enfin, parce que les conchyliologues ont tantôt restreint, ou tantôt augmenté le nombre des espèces, suivant leur fantaisie. Je connais un auteur qui, dans le même travail sur les Naïades, a admis au commencement un certain 
nombre de formes, puis les a éliminées au milieu, pour les reconstituer en partie à la fin, et cela, sans rime ni raison.

Chaque auteur, en un mot, a compris l'espèce à sa faȩon.

Il est vrai que sur ce sujet, on ne pourra jamais s'entendre.

Les uns croient à une forme primitive immuable.

D'autres nient la fixité de l'espèce et affirment que tout est mutation.

D'autres encore admettent une forme fixe avec des variétés constantes.

D'autres, enfin, ne reconnaissent que des races, que des variétés.

Je ne finirais point si je voulais noter toutes les nuances d'opinion.

Je tiens seulement à faire connaître ma pensée au sujet de l'espèce, pensée qui m'a toujours guidé dans mes travaux, et n'a été comprise que par quelques esprits impartiaux.

Pour moi, l'espèce (c'est-à-dire la réunion d'individus de même nature, de mêmes caractères, produisant des êtres semblables) est RELATIVE sous LA DOUBLE INFLUeNGE du TEMPS ET DES MILIEUX.

J'entends par là que cetté réunion d'individus, produisant des êtres semblables, produiront toujours des êtres absolument semblables, tant que les conditions climatologiques seront les mêmes, tant que les milieux où ils vivent ne subiront pas de changements.

J'entends encore par là que, si à la suite des temps, ou 
à la suite de changements de demeure, les milieux viennent à ne plus être les mêmes, ces êtres seront forcés de se sélecter des caractères spéciaux pour se mettre à même de soutenir la lutte vitale dans les conditions ou les milieux nouveaux où ils seront forcés de viure.

Je n'admets pas la fixité de l'espèce, de l'espèce telle qu'elle est comprise par les spécificateurs. Je la crois reltttive, parce qu'elle est subordonnée aux influences.

C'est pour cela que je distingue tout ce que la nature a caractérisé, alors que ces individus montrent une constance dans la succession de leurs caractères, ces caractères seraient-ils le résultat des milieux, du temps ou de toute autre cause.

Je passe, je le sais, dans l'esprit de bien des personnes qui n'ont pas saisi ma façon de penser, pour un auteur à système. Or, je suis peut-être le seul, justement, qui n'en ait point au sujet de l'espèce, puisque j'admets toutes les formes stables que produit la nature. Je ne me permets pas, comme bien d'autres, de réunir ce qu'elle a séparé, ou de séparer ce qu'elle a uni.

Je ne me préoccupe que d'une chose, de savoir si la forme que je décris est constante; alors, si je reconnais sa fixité, je la regarde comme espèce et je la publie comme telle. " D'ailleurs peu importe, a dit Draparnaud dans son prodrome, quel soit le nom que l'on donne à une réunion d'individus liés par des rapports de ressemblance et qu'on l'appelle espèce ou variété. L'essentiel, c'est qu'on en fasse mention et qu'on en décrive les caractères d'une manière exacte, claire et précise. D 
Ce n'est pas une monographie des Anodontes d'Europe que je donne actuellement.

Le moment n'est pas venu de faire une monographie des espèces de ce genre. Malgré les nombreuses formes que j'ai à signaler, je suis persuadé que je ne connais pas le quart des espèces qui existent et qui seront publiées dans une vingtaine d'années.

J'ai simplement l'intention de grouper les Anodontes et de donner, sur chacune de celles que je vais indiquer, les signes les plus indispensables pour les faire reconnaître. Pour celles qui ont été publiées, je me contenterai d'indiquer une bonne figure. Je ne donnerai les caractères complets que pour les espèces nouvelles. Si jagissais autrement, je serais entraîné trop loin; pour le moment je ne vois pas la nécessité de m'étendre trop longuement.

J'ai fait mon possible pour restreindre autant que je l'ai pu le nombre des espèces. J'ai agi, en cette circonstance, en conscience et sans parti pris. Malgré tout, cependant, j'ai été amené à reconnaître environ 200 espèces, ou farmes stables, que je ne puis faire autrement que de caractériser. Il y a trois ans, lors de ma Classification des genres et des familles du système européen, je n'en connaissais que quatre-vingt-sept.

Je sais parfaitement qu'il ne faut pas augmenter par trop le nombre des espèces, mais je sais également qu'il est prudent de ne pas les restreindre. Lorsqu'on se lance, en effet, dans cette voie, loin d'éclaircir la science, on ne fait que la plonger dans des confusions déplorables et presque inextricables. 
Je prends pour exemple l'Anodonta Pictetiana du lac de Genève.

Établie par un de ces amateurs sans érudition et sans coup d'oil, cette Anodonte comprenait primitivement un ensemble de cygnea, de cellensis, d'anatina, de tumida, etc.

L'auteur des Naiades de l'Aube, pour sa part (voir Rev. et mag. zoul.185\%4, p. 323 et 326), reconnaît que la Picletiana ne vaut rien puisqu'elle est composée de cellensis et de rostrata.

Brot (Naïades du Léman, 1867), d'un autre côté, avoue (p. 45) qu'il élimine, de cette espèce, une forme qu'il rapporte à la cygnea var. rostrata (qui entre parenthèses n'est pas la cygnea), mais qu'il regarde le reste (c'est-àdire trois variétés) comme constituant la vraie Picleliana.

Or, la première forme, ou variété (pour laquelle je conserve le nom de Pictetiana), est une espèce du groupe des Piscinales.

La deuxième appelée rostrata, est la tumida de Kuster du groupe des Brotiana.

La troisième, nommée elongata, est la Lacuum du groupe des Gilyciana.

Voilà donc une espèce qui renferme quatre formes de quatre groupes différents.

Tel est le résultat de la manie des réunions.

Les confusions, comme on le voit, deviennent déplorables. Il faut plus d'éludes el de patience pour se retrouver dans un pareil chaos, que si l'on avait simplement caractérisé d'une manière exacte et précise, comme le dit Draparnaud, les formes stables, telles que les produit la 
nature. Le nombre iles formes aurait augmenté, il est vrai, mais la science aurait gagné en clarté.

Il ne faut pas d'arbitraire. On doit suivre la nature. C'est le meilleur guide.

Le mémoire sur les Naïades du Léman me rappelle de point en point la Monographie des Succinées du Dr Baudon. Dans ces deux travaux, on reconnaît de bonnes observations, des descriptions faites avec conscience, d'excellentes figures; malheureusement on n'y constate que de fausses déterminations.

Ainsi, dans les Nä̈ades du Léman (puisque c'est le seul travail dont j'ai besoin de m'occuper en ce moment), voici les déterminations des espèces figurées et les noms que ces mêmes Anodontes doivent porter en réalité.

Pl. I.

$-\quad$ II.

- III.

- IV.

- V.f.1.

f. 2.

- VI. f. 1 et 4

tata.

- abbreviata.

- VII.f.1 et 3. - Anatina major et rostrata - (espèces inconnues du groupe des piscinales )

f. 2. - Anatina monstrosa.... - illuviosa.

- VIII.f. 1. - Pictetiana........... - (c'est cette forme que j'ad o pte pourla Pictetiana).

f. 2. - Pictetiana rostrata.... - tumida.

1. 3. - Pictetiana elongata.... - lacuum. 
Si je passe aux planches des Naïades de l'Aube, je trouve également de fausses déterminations.

Pl. I. Anod. Cygnœa............ Anod. pammegala (type).

- II. - Cellensis.......... - arenaria (peu typi-

- III. f. 1. - Complanata $\} \ldots\{-$ que.)

f. 2. Elongata. $\ldots . .$. - danodontes).

- IV. f. 1. - Anatina typus...... - nycterina.

C. $2 . \quad$ - Parvula............ - (hien).

- V.f.1. - Piscinalis.......... - subrhombea.

I $2 . \quad$ Rostrata............ - Jourdheuili(echant. à rostre un peu

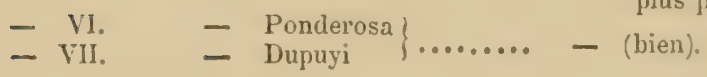

Il résulte de l'examen des espèces décrites par ces deux auteurs que presque toutes leurs Anodontes sont mal nommées et qu'ils ne connaissent ni la cygncea, ni l'anatina.

Il est vrai que ces deux espèces, les premières créées, sont des formes complètement inconnues, sauf de quelques auteurs. Linnæus, du reste, les a si imparfaitement caractérisées, si mal définies, qu'il n'y a rien d'extraordinaire à ce qu'elles n'aient pas été comprises.

Chaque auteur s'est créé une forme à sa fantaisie et l'a présentée comme le type linnéen.

Je ne pouvais, daus ces Matériaux, agir à ma guise, à l'instar de mes prérlécesseurs, je devais rechercher ce que pouvaient ĉtreces deux Anodontes. Pour arriver à cette connaissance, je me suis adressé à l'excellent travail d'Hanley.

Hanley, le savant conchyliologiste anglais, l'auteur avec Forbes, de la meilleur malacologie marine, terrestre et fluviatile qui ait été composée sur l'Angleterre, dans 
l'intention de rendre un grand service à la science, a pris à tâche d'étudier avec le plus grand soin la collection de Linnæus.

Cette collection, acquise autrefois par la Société Linnéenne de Londres, et qui a été religieusement conservée, est la collection type du Systema naturæ.

Or, Hanley a résumé ses études et condensé ses observations dans ses "Ipsa Linxni conchylia. - The shells of Linnous determined from his manuscrits and collections 》 (London, 1855, 1 vol. in-8 av. 5 pl. col. sur lesquelles sont représentés les types les moins connus.)

Au sujet de la cygncea, Hanley affirme (p. 144) que la figure 280 de l'iconographie de Rossmässler, nommée cellensis, est le véritable Mytilus cygneus de la collection du Systema natura.

Au sujet de l'anatina, cet auteur croit reconnaitre cette espèce dans la complanata (fig. 283) de Rossmässler; mais, pour plus de sûreté, il a pris le soin de donner (pl. 2, f.1) une représentation exacte du Mytilus anatinus.

Hanley a eu raison de donner cette figure, qui est excellente, parce qu'elle montre non une complanata, mais une Anodonte en forme de fer de lance, écourtée, du groupe de la Rayi. Parmi les nombreuses espèces représentées dans l'iconographie de Rossmässler, celle qui se rapproche leplus de l'anatina de Hanley est l'Anodonte figurée 417.

Pour moi, la cygnæa est celle 280 et l'anatina celle 417 de l'iconographie.

Toutes les autres cygncea et ariatina, figurées dans les ouvrages, même dans Rossmässler, qui ne se rapportent pas, 
non pas par ì peu près, mais exactement à ces deux figures 280 et 417, we sont, à mon sens, ni des cygnea ni des anatina.

Les caracteres du genre Anodonte sont connus. Ils ont été donnés par un grand nombre d'auteurs. Je n'en parlerai pas.

Je dirai seulement qu'il existe chez les Anodontes deux sortes de ligaments (comme chez toutes les Naïades, ainsi que l'avait fort bien remarqué Lamarck). D'abord un ligament postérieur, externe; puis un autre, antérieur, semblable à une membrane, s'étendant de l'extrémité du bord antéro-dorsal jusqu'aux crochets et se prolongeant ordinairement au delà sous. le ligament postérieur.

Ces deux ligaments, dans l'état de jeunesse, sont recouverts par le test. A l'état adulte (le test se brisant), ils deviennent externes. Chez certains groupes, cependant, comme chez celui des piscinales, par exemple, le test persiste et recouvre ordinairement le ligament postérieur, qui, dans ce cas, se trouve symphynoté.

Les Anodontes vivantes d'Europe appartiennent à 28 groupes difl'érents.

A chacun de ces groupes, j'ai donné, suivant mon habitude, le nom de l'espèce la plus ancienne ou la mieux caractérisée.

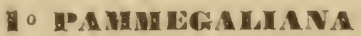

Les especes de ce groupe, toutes très ventrues, à valves relativement minces, peu pesantes, sont caractérisées par une forme écourtée, plus ou moins oblongue-subarrontie, 
ordinairement très convexe, vers le bord palléal (CONvEXITÉ PRESQUE MÉDIANE, pammegala, eucypha, — OU ANTÉRIEURE, stagnalis, gravida, - ou PostéRIEURE, eumorplia, Thiesse, ou enfin, Centrale PEU accentuée, callidora).

Les Anodonta taurica et Apollonica, que j'ai décrites au chapitre précédent, appartiennent à ce groupe.

\section{Anodonta pammegala,}

Cette espèce, une des plus belles et des plus grandes du système européen, dont il est inutile de donner une description, a été parfaitement représentée :

$1^{\circ}$ Par Schroter (Flussconch. Thuring. pl. 1, f. 1, 1779), sous le non ( $p .159)$ de musculus maximus planior viridescens edentulus.

20 Par Rossmässler (Iconogr. V et VI, 1837, pl. 25, f. 342), sous l'appellation d'An. cygnœea.

$3^{\circ}$ Par Kuster (Anod, in : $2^{\circ}$ édit. de Chemnitz, pl. 13 , 1852), également sous le nom de cygnaca, et, enfin, sous le même vocable, par l'auteur des Naïades de l'Aube (pl.1).

Cette Anodonte, considérée jusqu'à présent comme la cygncea, type de Linnæus, bien qu'elle n'ait aucun rapport avec la figure 280 de Rossmässler, est surtoul caractérisée par la dilatation et la convexité de son bord inférieur.

Cette magnifique espèce (long. 200 à 225. Haut. 120 millim.), paraît spéciale à l'Europe centrale, où elle semble assez abond ante dans les étangs de la Saxe et de la Bavière. En France, je ne la connais que des étangs de Villemereuil et de Gerusdots, près de Troyes, où elle est excessivement commune. 


\section{Anodonta encypha.}

Celte nouvelle espèce, si distincte de la pammegala, par sa taille moindre, par sa forme plus ventrue, plus écourtée (surtout dans sa partie postérieure), moins rostrée, moins convexe au bord palléal, a été aussi prise pour la cygnœa de Linnæus.

Cette Anodonte a été représentée, sous ce nom, par Rossmässler (Iconogrr. I, 1833 fig. 6i 7 ), et par l'abbé Dupuy (Hist. Moll. France (4e fasc., 18\%0), pl. XV, f. 14).

Ces deux figures suftisent amplement pour la connaissance de cette espèce.

L'eucippha habite dans les étangs et les eaux vaseuses de l'Allemagne et du Danemark, d'où je l'ai reçue de Mörch.

En France, elle vit, à ma connaissance, dans les bassins du parc de Rambouillet (Nabille), et, aux environs de Fontenay-le-Comte, en Vendée (Tac. Letourneux).

Cette espèce est peu commune. L'abbé Dupuy dit qu'il l'a reçue trois fois : une fois de Terver, comme provenant de la Saône; une autre fois, d'une localité inconnue; enfin, une troisième, des environs de Troyes. - Pour moi, je ne la connais ni de Troyes ni du département de l'Aube.

\section{Anodonta stagnalis.}

Mytilus stagnalis, Sowerby, Brit. misc. pl. 16 (teste Brown), et Anodon stagnalis, Brown, illust. conch., p. 79, pl. 28, f. 2, et Illust. of the land and fresh wat. conch. Brit. and Ireland, 1845, p. 102, pl. XIV.

Magnifique espèce, à région antérieure biendéveloppée, 
arrondie, à bord inférieur bien convexe, caractérisée surtout par une région postérieure s'atténuant assez brusquement et se terminant par un rostre très prononcé.

La stagnalis offre en exagéré les caractères de la gravida de Grèce.

Cette Anodonte, très bien figurée dans les illustrations de Brown, vit dans les canaux et les étangs d'Angleterre, notamment à Bolton Bridge dans le Lancashire, à Kew, sur la Tamise, dans le Surrey, etc. M. Coutagne vient de la recueillir aux environs de Lyon.

\section{Anodonta gravida.}

Anodonta gravida. Drouët, in Journ. conch., p. 142, 1879.

Cette Anodonte du lac Copaïs, en Grèce, bien que ressemblant beaucoup comme forme à la stagnalis, mérite néanmoins d'être conservée comme espèce.

\section{Anodonta eumorphia.}

Anodonta eumorphia. Letourneux, in litt.

Long. $\max . . . \ldots \ldots \ldots \ldots \ldots . . . . .115$ mill.

Haut. $\max . . . \ldots \ldots \ldots \ldots \ldots . . . .672$ -

Epaiss. nnax. (à 42 dessommets, - à 55 du rostre, - à 59 du bord ant., à 41 de l'angle postéro-dorsal, - à 32 de la base de la perpendiculaire). 46 -

Long. de la crête ligamento.dorsale des sommets à l'angle postéro-dorsal... 36 - 
Dist. de cet angle au rostre........ 60 mill.

Corde apico-rostrale............. 87 -

Haut. de la perpendiculaire........ 66 -

Dist. de cette perpend. au bord antér.. 40 -

- du même point au rostre postérieur.................

$751 / 2$

- enfin, de la base de la perpend. à

l'angle postéro-dorsal....... 73 -

Cette espèce est caraclérisée par une forme oblongue descendante, à partie postérieure dilatée, et, par une grande convexité, dont le maximum (46 mill.), presque à égale distance des extrémités antérieure (59 mill.) et postérieure (气̆ mill.), se trouve très éloigné des sommets (42 mill.) et de l'angle postéro-dorsal ( 41 mill.). Cette convexité maximum ainsi placée donne, à la région postérieure, un aspect ventru tout particulier. Ghez les staynalis et gravida, la convexité est plus portée vers la région antérieure et moins éloignée des sommets. Cette région est, en outre, plus dilatée, plus ventrue que la postérieure, qui s'atténue en rostre. C'est l'inverse chez l'eumorplia, où la région postérieure, très dilatée, se termine en un rostre anguleusement arrondi, dont l'extrémité est légèrement ascendant. Chez cette Anodonte, le maximum de la hauteur se trouve presque à l'extrémité du ligament externe, tout proche de l'angle postéro-dorsal, tandis que chez les stagnalis et gravida, ce même maximum se montre au niveau de la perpendiculaire; or, chez l'eumorphia, il y a une distance de 33 mill. entre la perpendiculaire et la ligne de la hauteur maximum.

Coq. de taille moyenne (115 de long. sur 72 mill. de haut.), de forme oblongue-descendante, rétrécie antérieu. 
r'ement, dilatée dans sa région postérieure, très convexe (convexité assez inférieure).

Valves très bâillantes : $1^{\circ}$ dans toute sa région palléale; $2^{\circ}$ de l'angle postéro-dorsal jusqu'au rostre.

Bord supérieur presque rectiligne. Région antérieure médiocre, bien ronde. Bord inférieur descendant, faiblement convexe. Région postérieure presque le double plus longue que l'antérieure, dilatée, se terminant par un large rostre à contour anguleusement arrondi ( 3 angles), dont l'extrémité est un tant soit peu remontante. Entre ce rostre et l'angle postéro-dorsal, la ligne du contour suit une direction oblique recto-descendante, très légèrement concave vers le rostre, par suite de l'extrémité rostrale faiblement ascendante.

Grête ligamento-dorsale médiocrement dilatée, comprimée assez brusquement vers l'angle postéro-dorsal et passablement amincie en cet endroit.

Arête dorsale bien accentuée, présentant deux lignes divergentes aboutissant aux parties anguleuses du rostre.

Sommets r'enflés, volumineux (crochets aigus), arrondis, sillonnés par de petites rides ondulées assez saillantes.

Stries d’accroissement émoussées, très feuilletées sur toute la ligne des contours, notamment sur la partie postéro-dorsale.

Épiderme très brillant (sauf sur les parties feuilletées), d'une belle teinte verte radiée, passant à une nuance grisecendrée, foncée vers la région supéro-médiane, et à un ton rouge ochracé sur les sommets.

Intérieur d'une belle nacre irisée blanche-bleuacée. Ligament postérieur épais, court (long. 28 mill.), presque entièrement recouvert par le test. 
Cette espèce a été découverte, en Slavonie, par notre ami le conseiller Letourneux, dans les marais de la Drave, près d'Esseg.

\section{Anorionta Thiessae.}

Cette Anodonte, à laquelle j'attribue le nom de mademoiselle Thiesse de Chalcis (Eubée), m’est parvenue sous le nom erroné de gravida.

\begin{tabular}{|c|c|c|c|c|}
\hline & Thies & & Grav & \\
\hline Long. max.. & 125 & $»$ & 131 & » \\
\hline Haut. $\max . . . \ldots \ldots \ldots \ldots$ & 74 & 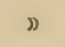 & 76 & ” \\
\hline $\begin{array}{l}\text { Epaiss, max. (à } 45 \text { des sommets; } \\
\text { à } 50 \text { du rostre; à } 691 / 2 \text { du } \\
\text { bord antér.; à } 30 \text { de l'angle } \\
\text { postéro-dorsal; à } 40 \text { de la base }\end{array}$ & & & & \\
\hline de la perpend.)........... & 50 & $1 / 2$ & 50 & $»$ \\
\hline 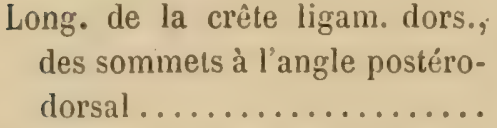 & 44 & ) & 45 & ") \\
\hline Distance de cet angle au rostre.. & 58 & ) & 66 & $1 / 2$ \\
\hline Corde apico-rostrale......... & 94 & ) & 104 & 》 \\
\hline Haut. de la perpendiculaire.... & 70 & 》 & 76 & » \\
\hline $\begin{array}{l}\text { Dist. de cette perpend. au bord } \\
\text { antér.................... } \\
\text { Dist. du même point de cette }\end{array}$ & 40 & $1 / 2$ & & $1 / 2$ \\
\hline & 80 & $1 / 2$ & 90 & ” \\
\hline $\begin{array}{l}\text { Enfin, de la base de la perpend. } \\
\text { à l'angle postéro-dorsal..... }\end{array}$ & 76 & 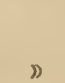 & 82 & (1) \\
\hline
\end{tabular}

Je viens de donner la mensuration exacte de cette Ano. 
donte comparativement avec celle de la gravida, d'après un échantillon un peu plus petit que celui décrit par le sieur Drouët, échantillon mesurant 140 de longueur, 80 de hauteur et $ّ \preceq$ d'épaisseur.

Or, d'après cette mensuration, l'on remarque que la Thiessce est moins allongée, moins haute, bien qu'également aussi ventrue; on remarque de plus que la partie antérieure est plus développée, puisqu'elle a $40 \%$ 1/2 (la gravida $391 / 2$ ) et que sa région postérieure est plus écourtée, puisqu'elle atteint seulement $801 / 2$ au lieu de 90; enfin, qu'au niveau de la perpendiculaire, elle est moins haute que la gravida, puisqu'elle n'a que '70 au lieu de 76 millim. Chez la gravida, la hauteur maximum (76 mill.) se confond avec celle de la perpendiculaire, tandis que chez la Thiessce, la hauteur maximum (74 mill.) se trouve située à 23 mill. en arrière de la perpendiculaire, qui n’a que 70 mill. Ces différences de mens' ration donnent à la Thiess $x$ un aspect très dissemblable de celui de la gravida.

Chez la Thiesse, bien que les sommets soient plus médians et moins rapprochés du bord antérieur que ceux de la gravida, la région antérieure paraît moins dilatée, surtout dans le sens de la hauteur; parce que, chez cette espèce, la dilatation caractéristique qui a lieu chez la gravida à la partie antéro-palléale, n'existe pas chez elle en cet endroit, mais se trouve reportée en arrière de la per pendiculaire, presque au niveau d'une verticale tirée de l'angle postéro-dorsal. Cette dilatation postérieure, en changeant complètement le mode de ses contours, donne à la Thiessce un aspect tout différent de celui de la gravida. Par suite de cette dilatation, en effet, le rostre, plus écourté et plus brièvement aigu, paraît plus remontant, avec un contour supérieur sinué. La convexité maximum,

MA1. 1881. 
qui chez la gravida est plus centrale, semble, chez la Thiessa, plus rapprochée du rostre et du bord palléal, d'où il résulte que la région antérieure au lieu de paraître gonflée, comme celle de la gravida, s'atténue, au contraire, d'une façon fort sensible, en s'amincissant ver's le bord du contour antérieur.

Coq, de forme ovalaire très rentlée, à valves minces non bâillantes. Bord supérieur arqué. Région antérieure arrondie, non dilatée dans le sens de la hauteur. Bord inférieur convexe-descendant jusqu'à environ 30 millimètres en arrière de la perpendiculaire, où il offre une dilatation prononcée, puis remontant presque en ligne droite vers le rostre. Région postérieure médiocrement allongée ( 80 mill. 1/2 contre $451 / 2$ pour le côté antérieuri), terminée par un rostre court, prononcé, et offrant en dessus, entre le rostre et l'angle postéro-dorsal, un contour sinué.

Sommets peu renflés, comme écrasés, assez médians (à $451 / 2$ du bord antérieur), sillonnés par de fortes sinuosités concentriques, légèrement rugueuses-tremblotées. Arête dorsale saillante, très prononcée, subbianguleuse, descendant presque brusquement sur la crête dorsale qui est peu développée.

Stries d'accroissement bien marquées, très saillantes comme liratiformes en certains endroits, enfin, paraissant sur' la partie postérieure de l'arête dorsale légèrement feuilletées.

Epiderme très brillant, d'un ton ochracé brunâtre avec des nuances plus chaudes sur la convexité ombonale, puis offrant des zones concentriques jaunacées ou verdâtres ver's la région des coulours. 
Intérieur d'une nacre bleuâtre bien irisée, présentant vers la région des sommets des tons rosacés.

Ligament antérieur excessivement mince, se prolongeant à 10 millimètres en arrière des crochets, qui sont fort aigus. Ligament postérieur court, peu saillant, presque toujours symphynoté, se terminant par une lunule étroite très allongée.

Charnière subrectiligne, subfiliforme, offrant seulement en arrière des crochets un léger renflement allongé un peu en forme de gouttière. Impressions superficielles, très irrégulières et très largement développées.

Cette Anodonte a été découverte par mademoiselle Thiesse, dans le lac Copaìs (Grèce), où elle vit en compagnie de la gravida, avec laquelle elle avait été confondue.

\section{Anodonta callidaea.}

Anodonta callidæa, Letourneux, in litt.

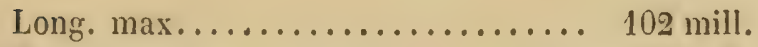

Haut. $\max . \ldots \ldots \ldots \ldots \ldots \ldots \ldots$ ๖ํ....... $1 / 2$

Epaiss. max. (à 31 des sommets, - à

วั5 du rostre, - à 46 du bord antér.

- à 31 1/2 de l'angle post. dors.,

- à 29 de la base de la perpend.). .

Longueur de la crête ligam. dors, des sommets à l'angle postéro-dorsal... $361 / 2$

Distance de cet angle au rostre...... 47 -

Corde apico-rostrale............. 79 -

Haut. de la perpendiculaire........ 55 - 
Dist. de cette perpend. au bord antér. $\quad 33$ mill.

- du même point de cette perpend.

at rostre.............. ii -

- enfin, de la base de la perpend. i

l'angle postéro-dorsal....... 60 -

Cetle Anodonte, la plus petite que je connaisse de ce groupe, est une espèce remarquable par son peu de hauteur comparé à sa longueur, et, par sa forme oblongueallongée, relativement très convexe. Chez cette espèce la convexité maximum ( 39 mill.), plus rapprochée du bord antérieur, est presque à égale distance des sommets et de la base de la perpendiculaire. Sa régrion antirieure, bien développée, est aussi haute (à un demi-millimètre près) au niveau de la perpendiculaire, qu'à celui de l'extrémité du ligament postérieur dont la hauteur maximum n'est que de $5051 / 2$. Chez cette Anodonte, la lunule du ligament est très allongée (13 mill.), tandis que chez l'eumorphia, qui est d'une taille supérieure, la lunulc correspundante n'a que 8 mill.

Coquille de forme oblongue-allongée, très convexe, it valves très bâillantes : $1^{\circ}$ à son bord antéro-palléal; yّo en dessous de l'angle postéro-dorsal.

Bord supérieur arqué, assez court. Région antérieure médiocre, bien arrondis. Bord inférieur faiblement convexe-descendant, se relevaut en courbe vers le rostre. Rérgion postérieure allongée, près de deux fois plus longue que l'antérieure, terminée par un rostre prononcé, à contour arrondi, et, offrant en dessus, entre le rostre et l'angle postéro-dorsal, une direction ligèrement concave.

Sommets renflés, arrondis, très ventrus, peu proéminents, sillonnés de petites lignes tremblotées. Crochels trìs aigus. Arête dorsale très convexe. Crête dorsale 
presque exiguë, à peine dilatée, peu comprimée, faisant partie, pour ainsi dire, de la convexité de l'arête dorsale.

Stries d'accroissement émoussées, çà et là un peu plus fortes, feuilletées seulement sur la région supéro-dorsale et faiblement sur le contour antérieur.

Epiderme des plus brillants, d'une belle teinte jauneverdâtre, cendrée vers la région des sommets et ochracérougcùtre vers les crochets; enfin, orné d'une quantité de radiations vertes très accentuées.

Nacre intérieure d'une nuance blanche-bleuacée, très irisée.

Ligament antérieur, interne, lamelleux, très allongé, fort, épais, occupant toute l'épaisseur de la charnière et se terminant sous le ligament postérieur à 8 mill. en arrière des crochets.

Ligament postérieur, externe, très court, excessivement robuste et proéminent, terminé par une très grande lunule allongée.

Charnière un peu arquée. Région cardinale occupée par le ligament antérieur. Région latérale peu allongée, épaisse, d'une nacre blanchs d'un ton mat.

Impressions bien accentuées, très irrégulières, le plus souvent spathuliformes.

Celte Anodonte habite, en Croatie, dans un torrent audessus de Pregrarla, près de Krapina (Letourneux).

\section{VENTHICOSIANA}

Les mollusques de ce groupe sont tous, sauf les regularis et Livronica, de grandes espèces ventrues, allongécs, à stries d'accroissement généralement saillantes, souvent liratiformes. Elles peuvent facilement se liviser en trois séries : 
$1^{\circ}$ Ventricosa, cordata, Forchhammeri.

20 Gallica, Locardi, Charpyi, lirata et fragillima.

$3^{\circ}$ Stataria, regularis et Livronica.

Les espèces de la première série sont toutes les trois remarquables par la grande convexité de leur région ombonale. La ventricosa a ses bords supérieurs el inférieurs presque paralièles. La cordala, bien que possédant également des bords à peu près parallèles, est fortement sinuée à sa partie inférieure; enfin, la Forchhammeri, caractúrisće par un grand développement de sa partie antérieure, a ses bords convergents vers le rostre.

Les Anodontes de la seconde série se distinguent par une convexité arquée d'une façon régulière, des crochets au bord palléal, et, dont le maximum de renflement, presque médian, ne s'accentue pas, comme chez les formes de la première série, seulement à la région ombonale. Cette convexité est plus ou moins prononcce suivant les espèces. Ainsi, de très accentuée chez les Gallica et Locardi, elle s'atténue en passant par las formes Charpyi, lirala jusqu'à la fragillima qui est la moins convexe.

La gallica, régulièrement renflée dans toutes ses parties, a ses bords (supérieur et inférieur) presque parallèles. Chez la Locardi, les bords convergent vers le rostre, par suite d'une plus grande hauteur à sa région antérieure. Ciette espìce joue un peu dans cette série le rôle que remplit la Forchhammeri dans la série précédente. La Charpyi, offre à peu près les mêmes lignes de contour; seulement celle.ci moins ventrue, sillonnée de côtes grossières et irrégulières, est relativement, à cause de sa forme écourlée, plus haute que la Locardi. La lirata, très voisine de la Charpyi, est surtout remarquable par ses stries liratiformes bien régulières. Quant à la fragillima, la moins 
convexe de cette série, ses bords (supérieur et inférieur) au lieu d'être parallèles ou plus ou moins convergents vers le rostre, offrent au contraire une tendance divergente, par suite d'une plus grande dilatation en hauteur de sa région postérieure.

Les espèces de la troisième et dernière série sont ovalaires-allongées, à bords (supérieur et inférieur) aussi arqués l'un que l'autre, et, dont la plus grande hauteur se trouve presque juste à égale distance des extrémités. La convexité, chez ces Anodontes, est régulière des sommets au bord palléal. La stataria, la plus grande et la Iivronica la plus petite, sont bien rostrées. La regularis est, au contraire, postérieurement très obtuse.

Les Ventricosiana sont des formes qui paraissent préférer les eaux vaseuses de la France et de l'Allemagne du Nord, La fragillima, plus méridionale, s'étend en Bavière, en France, jusqu'en Italie. La regularis, du centre hispanique, n'est connue que du Portugal.

\section{Amodonia rentricosa.}

Anodonta ventricosa, C.rPfeiffer, naturg. Deutsch. Moll. II, 1825, p. 30, pl. 3, f. 4 (seulement). - (Non, Anod. ventricosa de tous les autres auteurs).

Je considère la figure 4 comme représentant cette espèce, parce que, dans l'esprit de C. Pfeiffer, cette figure seule donne la représentation de celte Anodonte à l'élat adulte, tandis que les autres, d'après cet auteur, ne sont que des jeunes de cette espìce. 
Or, les trois ćchantillons (fig. 1, 2 et 3 ) que Pfeifier a fait figurer comme des ventricosa non adultes, ne sont pas des jeunes de cette espèce.

Il existe, en effet, de grantes difiérences entre la ventricosa adulte et ces soi-disant ventricosa non adultes.

La ventricosa type est une forme très oblongue-allongée, assez ventrue, à bords supérieur et inféricur un tant soit peu parallèles. Son arête dorsale offre une tendance à pré. senter une légère courbe dont l'extrémilé est un peu ascendante. La crête dorsale très allongée, ne dépasse jamais les sommets. Les stries d'accroissement, à l'élal jeune, offrent exactement en petit les mêmes contours qu'ù l'état adulte. Je puis certifier ce caractère, parce que je l'ai vérifié sur un grand nombre d'échantillons de tous les âges.

Or, les trois ventricosa non adultes (fig. 1, 2 et 3) que C. Pfeiffer a pris pour le jeune âge de cette espèce sont des Anodontes de forme triangulaire, à crête amincie, élevée, dont l'arête dorsale est régulièrement descendante. Les stries d'accroissement, chez ces trois échantillons, offrent un contour également subtriangulaire qui n'a aucun rapport avec celui des stries de la ventricosa (fig. 4). On n'a qu'à comparer ces figures les unes entre elles pour rester convaincu de ce que j'avance. En somme, ces échanlillons, qui, loin d'être des jeunes ventricosa, ne font pas même partie de son groupe, appartiennent à celui de la Spengleri, dont je parlerai plus tarl, groupe voisin de celui de la piscinalis. C'est sans doute la forme subtriangulaire de ces trois échantillons qui a frappé Kreglinger, et, qui a amené cet auteur à considérer (Binn. moll., p. 334, 1870) la ventricosa de C. Pfeifier comme une variété de la piscinalis.

La figure 4 représentant la ventricosa est assez exacte. 
Cette figure suffit pour la connnissance de cette espèce. D'après cette gravure, cette Anodonte a 137 de longueur sur une hauteur maximum de 68 millimètres. Je connais des échantillons qui atteignent 197, sur 84 de haut et 58 millimètres d'épaisseur.

La ventricosa est une forme du nord de l'Allemagne où elle a été découverte aux environs de Cassel dans l'ancienne Hesse électorale, et, au sud du Hanôrre, dans les rivières de la petite principauté de Schaumbourg-Lippe. En France, je la connais typique des canaux de Villemereuil, près de Troyes (Aube), de la Vesle à Limé dans l'Aisne (Mabille) et des viviers de Saint-Simon près de Toulouse (Fagot et Saint-Simon).

Un assez grand nombre d'auteurs ont mentionné, décrit ou même figuré des Anodontes sous le nom de ventricosa de C. Pfeiffer. Aucune de ces ventricosa n'est la vraie.

Ainsi, la ventricosa de l'abbé Dupuy (Noll. France, $6^{\circ}$ fasc. 1852. p. 603, pl. XVI, fig. 13), signalée du nord de la France, n'est pas cette espèce, mais l'acyrta forme que je rapporte, comme variété maxima, à l'A nodonta fragillima de Clessin.

La ventricosa de Kuster (Anod,, p. 56, pl. XIA , f. 3-5), de la provenance du sud du Wurtemberg, se compose de deux espèces, dont l'une (fig. 3) est une forme du groupe de l'arealis, l'autre (fig. 4 et ö) de celui de la Glyca.

La ventricosa de Joannis (Étude naiades in: Ann. Soc. Linn. Naine-et-Loire, JII, 1858, p. 270, pl. 8) est une forme à bord inférieur bien convexe, qui m'est inconnue.

Quant à la ventricosa de Drouët (Étude Naïades France, II, 18ว2, p. 9), c'est une réunion de tout ce que l'on veut. 


\section{Anodonta corlata.}

Anodonta cellensis, var inflata Rossmässler, Europ. nayad. in : Zeitschr. malak., p. 151853.

Anudonta cygnoea, var. cordata, Rossmïssler, Iconogr. (17 et 18,1859$)$, p. 136 , fig. 968 .

Grande et magnifique espèce (long. 198, haut. 96, ép. 72 millim.), à sommets très bombés, à valves épaisses trìs convexes, à stries d'accroissement çà et là saillantes et liratiformes, à surface d'une teinte noirûtre, ou souvent d'un beau vert uniforme; enfin, caractérisée par une sinuosité très prononcée vers les troisquarts postérieurs du bord palléal.

Cette Anodonte a été trouvée dans un petit marais très profond, à fond vaseux, prìs du village de Platschutz, non loin d'Altenburg; en Allemagne. J'en possède deux beaux échantillons bien typiques des canaux du château des Cours à Saint-Julien, près de Troyes (Aube).

\section{Anodonta Forchhammeri.}

Anodonta cygnoea, var. 2. Forchhammeri, Mörch. syn. Moll. Danix, p. 84, 1864.

Grande espèce (long. 190, haut. 100, ép. 60 mill.), à valves minces pour sa taille, brillantes, d'une teinte olivåtre foncée avec des zones pius obscures, rarement ver-

1. Non, Anod, inflata, du major Le Counte, in : Lea, syn. fam. nayad., p. 51, 1852 . 
dâtres, et, passant à une nuance jaunacée-rougeâtre vers les sommets. Stries d'accroissement saillantes vers les contours, ressemblant souvent à de grosses zones cordées, séparées les unes des autres par des sillons parfois profonds. Le caractère principal de cette espèce consiste en un grand développement de toute la région antérieure qui est relativement énorme, en comparaison de la région postérieure, qui diminue régulièrement pour se terminer par un rostre arrondi assez médiocre. Ainsi, la hauteur maximum coïncide avec la perpendiculaire, et, à partir de cette ligne, la région postérieure diminue très promptement. Au niveau de l'extrémité du ligament, la hauteur n'est plus guère que de 80 à 85 millim.

Cette espèce est pour le groupe de la ventricosa, ce qu'est la stagnalis pour celui de la pammegala.

J'ai reçu cette Anodonte de différentes localités du Danemark (Mörch), où elle vit dans des canaux vaseux.

Je la connais encore parfaitement typique des viviers de Saint-Simon, près Toulouse (Fagot), du lac du parc de la Tête-d'Or à Lyon (Locard), des étangs de Ville-d'Avray, près de Versailles (Mabille), etc.

\section{Anodonia gallica.}

Celte Anodonte a été parfaitement et très exactement représentée, sous l'appellation erronée de Mytilus anatinus par le révérend Revett Sheppard (on two new Brit. spec. of Mytilus, in: Trans. Linn. Soc. London, XIII, 1820, pl. IV, f. 1, et, Chenu, traduct. Des Trans. Soc. Linn. Lond. p. 270 , pl. XXVI, f. 1, 1845). En examinant avec soin cette figure, on se rendra compte de la forme des contour's et 
de l'aspect de celte espèce, elle ne peut être mieux l'endue. Je rapporte encore à cette Anodonte, la cellensis de Brown (Illust. conch., pl. XII, f. 1, 1845) qui représente bien ta gallica.

Cette coquille, à laquelle j’applique le nom de gallica, parce qu'elle me parait très abondante dans les eaux vaseuses du nord de la France, bien qu'elle soit également très répandue en Angleterre et en Allemarne, a été trouvée, en notre pays, dans les fossés et les canaux vaseux de Chicheray et de Notre-Dame-des-Prés, près de Troyes (Aube). Ce sont des ćchantillons de cette localité qui ont été décrits par le sieur Drouët comme la ventricosa de l'abbé Dupuy (non, Pfeiffer).

Je connais encore la gallica des étangs de Brisemiche, près de Meudon et des viviers de Meudon, près Paris (Mabille); des étang de Trappes (Seine-et-Oise) et de Villed'Avray, près de Versaillez (Mabille); de l'étang du Ranceau, près de Saint-Saulge dans la Nièvre (Brevière) et de celui de Boisdeniers, près de Tours (Rambur).

Voici la mensuration de la gallica :

Long. max................. læä mill.

Haut. max.................

Épaiss. max. (a 43 des sommets; ì 79 du rostre et du bord antérieur; à 38 de l'angle postéru-dorsal; à 57 de la báse de la perpend.)...............

Long. de la crête ligam. dors., du sommet à l'angle postéro-dorsal........ ¿s -

Distance de cet angle au rostre....... 71 -

Corde apico-rostrale............. 121 - 
Haut. de la perpendiculaire......... 76 mill.

Dist. de cette perpend. au bord antér... $46 \quad-$

- Du même point de cette perpend. au rostre.................. 110 -

- Enfin, de la base de la perpend. à l'angle postéro-dorsal...........

D’apris ces mesures, on remarque $: 1^{\circ}$ Que le maximum de la convexité est à égale distance du rostre et du bord antérieur: 20 que la plus grande hauteur est également la même que celle de la perpendiculaire, bien que cette plus grande hauteur ait été prise presque au niveau de l'angle postéro-dorsal, à อ̌ö mill. en arrière de la perpendiculaire.

Coq. régulièrement convexe, des sommets au bord palléal, de forme ovalaire-allongée, à valves assez épaisses, baillantes à la partie inféro-antéricure.

Bord supérieur presque rectiligne. Région antérieure bien ronde. Bord inférieur rectiligne, un peu sinué à sa partie médiane, et, remontant, à son extrémité, vers le rostre. Région postérieure, plus du double plus allongée que l'antérieure, se terminant par un rostre obtus, parfois un tant soit peu remontant. Entre ce rostre et l'angle postéro-dorsal, la ligne du contour suit une direction oblique recto-descendante.

Aréte dorsale très obtuse, bien convexe, obscurément bianguleuse à son extrémité. Crête dorsale médiocre, non ailée ni amincie. Sommets convexes, très oblus (crochets aigus), sillonnés par de fines striations concentriques peu lamellées.

Stries d'accroissement émoussées sur la convexité, sail- 
lantes et obtuses vers la région des contours, même parfois liratiformes.

Épiderme brillant, d'une couleur généralement très foncée, préseniant des nuances olivâtres, noirâtres ou ochracées, suivant les échantillons. Intérieur bien irisé, d'une nacre ayant une tendance à présenter des tons orangers.

Ligament antérieur occupant toute l'épaisseur de la charnière, se prolongeant de 10 à 15 millimètres en arrière des crochets et offrant en avant(sur les vieux échantillons) une assez grande lunule antérieure. Ligament postérieur robuste, proéminent, avec une large lunule terminale de forme triangulaire.

Charnière ordinairement épaisse, souvent subdenticulée à la région cardinale et présentant parfois sur la régrion latérale une gouttière plus ou moins prononcée, suivant l'àge des échantillons. Sur les jeunes individus, cette gouttière n'est pas sensible.

\section{Anodonta Locardi.}

Cette espèce, que je dédie à M. Arnoud Locard, de Lyon, auteur d'un grand nombre d'ouvrages scientifiques très estimés, est une Anodonte bien constante dans sa forme. Elle se distingue de la Gallica par sa région antérieure plus développée dans le sens de la hauteur et par ses bords (supérieur et inférieur) non parallèles, mais convergeant vers la partie rostrale, qui est très obtuse: Chez la Locardi, la hauteur maximum se trouve juste au niveau de la perpendiculaire. Chez la gallica, à 5 ’̌ mill. en arrière, on retrouve la même hauteur maximum. Il résulte de ce fait que 
chez la Gallica, les bords (supérieur et inférieur) sont parallèles sur une étendue de 5̆ŏ millimètres, tandis que chez la Locardi, ils sont convergents à l'instar de ceux de la Forchhammeri, bien qu'à un dégré moindre. Ces différences dans la dilatation de la région antérieure et dans la direction de ses bords donnent à la Locardi un aspect tout à fait dissemblable de celui de la Gallica.

La convexité chez cette Anodonte, bien que régulière, est également un peu moins accentuée que celle de la Gallica.

Cette espèce est sujet à des variations au sujet de la taille. Les dimensions ordinaires de la Locardi sont : 13 د de longueur sur 92 de hauteur et 44 d'épaisseur. Or j'ai reçu en communication de M. Locard un magnifique exemplaire, provenant de la Saône à Lyon, qui mesurait 200 sur 104 de hauteur et 636 d'épaisseur.

Cet Acéphale, qui vit dans la Saône, a été recueilli, en outre, dans les fossés du fort de la vitriolerie à Lyon (Locard), dans la Vesle (Aisne) près de Limé (Mabille); dans les canaux vaseux de l'ancienne Seine près Verrières à 10 kilomètres en amont de Troyes (Aube), etc.

\section{Anodonta Charpyi.}

Anodonta Charpyi, Dupuy mss.

Cette forme, que j'ai reçue des rivières du Jura, sous cette appellation, est une coquille très diflicile à caractériser. Par certains rapports, elle a de faux air's de cariosa, par d'autres, elle se rapproche de la série de la Gallica, dans laquelle je la laisse jusqu'à nouvel ordre. 
C'est une espèce ovalaire, médiocrement allongée, à valves non bâillantes, d'une couleur uniforme olivâtre ou rougeâtre très foncée, passant au noir vers les contours et présentant une surface terne chargée de stries concentriques grossières, comme rugueuses, saillantes, séparćes par des sillons plus ou moins profonds. Les sommets sont toujours excoriés, et souvent la corrosion s'étend, comme une lèpre, sur une grande partie des valves qui ont un aspect sale et sombre.

Sa convexité est régulière des sommets au bord pa!léal ; l'arête dor sale descend en pente douce sur la crête li gamento- Jorsale, qui est fort médiocre; les bords supérieur et inférieur sont légèrement arqués; la partie postérieure se termine par un rostre très obtus, un tant soit peu remontant; sa taille paraît varier beaucoup, mais la forme la plus ordinaire atteint 130 sur 67 de hauteur et 42 d'épaisseur. La hauteur maximum coïncide avec la perpendiculaire.

Je connais cette Anodonte du Drugion dans le Doubs (Fago1), de la Saône et des étangs de la Clayette, dans le département du Rhône (Locard), des environs de Tours (Rambur) et des viviers de Saint-Simon près de Toulouse (Fagut).

\section{Anodonta Mrata}

Anodonta Cygnœea, var. B. lirata, Mörch, Syn. Moll. Dan. p. 83. 1864 .

Celte Anodonte, voisine comme forme et comme taille de la précédente, en diffère, cependant, par sa convexité plus reportée vers la région ombonale; par son épiderme 
lisse brillant, d'une belle tcinte verte-jaunacée, et surtout par ses valves sillonnées par le grosses côtes concentriques saillantes, liratiformes, séparées les unes des autres par des intervalles plus ou moins profonds.

Cette espèce vit dans les canaux vaseux du Danemark, à Lolland, Classenstrave, etc. - Elle a été recueillie, en France, dans les étangs de Rambouillet (Nabille), à Grépy en Valois, dans l'Oise (Fagot), et dans ceux de la Clayette, dans le département du Rhône (Locard).

\section{Anodonta fragillima.}

Anodonta mutabilis, var. fragilissima, Clessin, Anod. (in : Chemnitz, $2^{\mathrm{e}}$ édit.), p. 237 , - et Anodonta fragilissima, p. 280, pl. 87, f. $2,1876$.

Gette espèce, découverte en Bavière dans un bras du Regen, près de Regenburg (Ratisbonne), et que j'ai reçue parfaitement typique de l'étang du Merle, près de SaintSaulge, dans la Nièvre, sous le nom erroné de Cygnea junior, paraîl assez répandue.

Je la connais de divers étangs ou marais du nord de la France (sans indication de localités), de l'étang de Ploërmel dans le Morbihan, des environs de Tours (Rambur), de l'étang de la Clayette dans le Rhône et des marécages des bords de la Saône, à Saint-Laurent-d'Ain près de Mâcon (Locard), où les échantillons sont relativement très ventrus et peu typiques; enfin, des environs de Florence et du lac de Massaciuccoli près de Viareggio (I)el Prete).

Cette Anodonte varie beaucoup comme taille; l'échantillon du Regen en Bavière (je le crois peu adulte), figuré 
par Clessin, n'atteint que 113 de iong sur 633 de haut et 27 d'épasseur; ceux du Nerle dans la Nièvre, 123 sur 70 de haut et 35 d'épaisseur; ceux de Ploermel dans le Morbihan, 140 sur 78 de haut et 43 d'épaisseur; enfin, ceux d'Italie, 154 sur 80 de haut et 48 millimètres d'épaisseur.

Ces grandes différences de taille m' avaient amené primitivement à séparer cette espèce : $1^{\circ}$ en Anod. acyrta pour les grands échantillons relativement bien ventrus comme ceux d'Italie ou du bassin du Rhône ou de la Saône; $2^{\circ}$ en Anod. fragillima pour les exemplaires de petite taille et peu ventrus (Regen en Barière, Ploërmel dans le Morbihan et Saint-Saulge dans la Nièvre). Mais, après un examen plus approfondi, j'ai reconnu qu'il y avait de si grandes similitudes de forme et de signes distinctifs entre ces exemplaires si différents de taille, que j'ai fini par considérer mon Anodonta acyrla comme une variété maxima de la fragillima.

Voici la mensuration exacte de cette espèce, d'après un échantillon de l'étang du lierle, échantillon parfaitement identique (bien qu'un peu plus grand) à celui décrit par Clessin.

Long. max.................. 128 mi.l.

Haut. $\max . . . \ldots \ldots \ldots \ldots \ldots$. 70 .

Épaiss. max. (à 38 des sommets; à 67 du rostre; à 88 du bord antér.; à 36 de l'angle post. dors.; à 38 de la base de

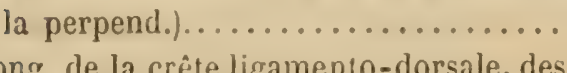

Long. de la crête ligamento-dorsale, des sommets à l'angle postéro-dorsal..... Distance de cet angle au rostre...... 58 - 
Corde apico-rostrale............. 96 mill.

Hauteur de la perpendiculaire....... $6{ }^{5}-$

Distance de cette perpend. au bord antér. $38 \quad$

- du même point de cette perpend. au rostre.............. 87 -

- enfin; de la base de la perpend.

à l'angle postéro-dorsal.... 73 -

D'après ces mesures, on remarque que la plus grande hauteur dépasse de 5 millimètres celle de la perpendiculaire. Cette hauteur maximum se trouve, en effet, à 36 millimètres en arrière, presque au niveau de l'extrémité du ligament postérieur. Il résulte de là que cette espèce possède une région postérieure plus dilatée que l'antérieure. Cette Anodonte, en effet, est caractérisée par un bord inférieur légìrement convexe-descendant, offrant vers son extrémité postérieure une dilatation cintrée très prononcée, dont le contour remonte ensuite d'une façon assez brusque vers le rostre, qui est un tant soit peu ascendant. La convexité est bien régulière. Lepoint maximum du renflement, faiblement postérieur, est à égale distance des sommets et de la base de la perpendiculaire. L'arête dorsale à peine saillante, est un tant soit peu arquée. Les sommets sont plats, comprimés, très finement sillonnés de petites zones concentriques. Les valves relativement très minces, assez fragiles, bien brillantes, d'une belle teirte jaunacée, postérieurement verdâtre, sont à peine bâillantes.

La figure donnée par Clessin fait bien comprendre la forme de cette espèce. Je n'ai pas vu, dans les ouvrages, d'autres figures que je puisse rapporter à cette Anodonte. 


\section{Amodonta stataria.}

Anodonta stata ia, Ray, in Litt. 1881.

La Stataria est fort bien représentée sous le nom de Cygnaza var. rostrata à la planche 2, fig. 1 de l'ourrage de Brot. Cette figure suffit à la connaissance de cette forme, qui se distingue de la Gallica : par son bord inférieur régulièrement convexe, par conséquent non parallèle avec le supérieur; par sa partie antérieure moins développée en hauteur, plus décurrente vers le bord palléal; par sa région postérieure un peu plus allongée et plus rostrée; par ses valves plus épaisses ne présentant inférieurement aucune apparence de sinuosité.

Chez la Stataria, la convexité parait plus antérieure, plus reportée vers les régions ombonale et postérieure. A partir du renflement maximum, la convexité s'atténue peu à peu régulièrement en forme de coin. Chez la Gallica, la convexité plus postérieure se conserve assez forte beaucoup plus loin et diminue d'une façon assez brusque seulement aux abords du rostre.

Cette Anodonte a été recueillie à l'extrémité du lac de Genève, devant les marais de Villeneuve, où elle est abondante. En France, elle a été rencontrée, identique à celle du Léman, dans les canaux de Villemereuil près de Troyes, dans l'Aube (J. Ray). 


\section{Anodonta regularis.}

Anodonta regularis, Morelet, Moll. Port. p.100, pl. X, $1840 ّ$.

Jolie espèce ovalaire, de taille médiocre (long. 100 à 125, haut. ( 3 à 66 mill.), d'une teinte d'un noir verdâtre ou rougeâtre, intérieurement bleuâtre, vivant dans les marais de la Taméga, près de Chavès, en Portuga!.

\section{Anodonta Luronica.}

Anodonta Livronica, Fagot, in Litt., 1881.

Cetle Anodonte qui vit dans le Livron, petite rivière du Jura, est une des plus jolies espèces du groupe des Ventricosiana. Cette coquille, d'une forme exactement oblongue, sans aucune sinuosité, est remarquable par son peu de hauteur comparé à sa longueur et par la grande régularité de l'atténuation de sa convexité vers les contours.

Long. $\max . \ldots \ldots \ldots \ldots \ldots \ldots \ldots \ldots .99$ mill.

Haut. $\max . \ldots \ldots \ldots \ldots \ldots \ldots \ldots$ is -

Épaiss. max. (à 26 des sommets; à 56 du rostre; à 43 du bord antérieur; à 39 de l'angle postéro-dorsal et de la base de la perpen 1.)............ 29 -

Long. de la crête ligamento-dorsale, des sommets à l'angle postéro-dorsal... 40 1/2

Distance de cet angle au rostre....... $42 \quad-$ 
Corde apico-rostrale............. 79 mill.

Haut. de la perpendiculaire........ 47 -

Dist. de cette perpend, au bord antér... 28 -

- du mème point de cette perpend. au rostre...................

- entin, de la-base de la perpend. à

l'angle postéro-dorsal....... 56 -

Coq. de forme oblongue, régulièrement convexe (convexité assez médiane, s'atténuant régulièrement sur les contours). Valves minces, à peine bâillantes à la partie antérieure.

Bord supérieur légèrement convexe, présentant exactement dans toute son étendue, le même contour que le bord inférieur. Région antérieure médiocre, bien arrondie. Région postérieure plus de deux fois plus longue que l'antéricure, s'atténuant avec une grande régularité, terminée par un rostre obtus un tant soit peu bianguleux et également distant des parties supérieure et inférieure.

Sommets trìs obtus, ventrus-arrondis, non proéminents, un peu écrasés, sillonnés par des stries concentriques tremblottíes assez robustes. Arête dorsale peu accentuée, perdue dans la convexité régulière des valves et présentant entre elle et la crête dorsale, qui est ni comprimée ni saillante, unı pente régulière.

Stries d'accroissement émoussées, peu saillantes, légèrement feuilletées vers la partie supéro-dorsale.

Epiderme presque toujours entièrement recourert par un encrassement calcaire très résistant et paraissant, lorsqu'il est enlevé, d'une teinte uniforme très foncée d'un cendré-fauve, passant sur les régions ombonale et postérieure à une nuance marron plus ou moins obscure. Nacre 
intérieure blanche, irisée de tons un peu bleuacés.

Ligament antérieur, interne, lamelleux, filiforme, se prolongeant à 8 ou 10 mill. en arrière des crochets. Ligament postérieur très allongé, peu volumineux, aux $3 / 4$ symphynoté, se terminant par une lunule allongée.

Charnière très mince, subrectiligne, sans caractère spécial. Impressions très superficielles.

Cette Anodonte, remarquable par son peu de hauteur comparé à sa longueur, ne peut être confondue avec aucune autre de ce groupe. La regularis, qui est celle qui se rapproche le plus de cette espèce pour sa taille, a une hauteur maximum de 66 sur 100 de longueur, tandis que celie-ci n'a que 48 sur 99.

\section{BPo GASTHOHIANA}

Je ne connais que deux espèce de ce groupe singulier. Ces espèces sont caractérisées par une convexité relativement énorme égalant ou dépassant même le tiers de la longucur, et, par une hauteur n'atteignant pas la moitiè de la longueur. II résulte de ces deux faits caractéristiquez que les Gasłrodiana sont des Anodontes excessivement bumbées, peu hautes, par conséquent allongées. Chez ces espèces, la région ombonale convexe-proéminente est très renflée-ven. true; les bords supérieur et inférieur sont presque parallèles, et la partie postérieure (de l'angle postéro-dorsal au rostre) est nettemment et fortement descendante, comine tronquée. 


\section{Auodonta Cyrtopiychia.}

Anodonta gibba, Held, in Clessin, gatt. Anod. (in $2^{\circ}$ édit. Chemnitz), p. 81, pl.14, f. 1-2, 1876.

J'ai été obligé de changer le nom de gibba, parce qu'il existe une Anodonte différente de celle-ci (voir Lea 185̆2) décrite par Benson.La Cyrtoptychia est admirablement bien représentée sur la planche XIV de l'ouvrage de Clessin. Je renvoie à cette figure pour la connaissance de cette espèce.

Cette Anodonte vit aux environs de Passau en Bavière, vraisemblablement dans le Danube. En France, je la connais bien caractérisée des environs de Tours où elle a été recueillie dans la Loire par le $\mathrm{D}^{\mathrm{r}}$ Rambur.

Amodonta Gastroda.

Long. $\max . . . . . . . . . . . . . . . . .996$ mill.

Haut. $\max . \ldots \ldots \ldots \ldots \ldots \ldots$. 45 .

Epaiss. max. (à 30 des sommets; à b̆l du rostre; à 47 du bord antér.; à 26 de l’angle postéro-dorsal; à 28 de la base de la perp.)..................

Long, de la crête ligam.-dorsale, des sommets à l'angle postéro-dorsal....... 38 -

Dist. de cet angle au rostre......... 48 -

Corde apico-rostrale............. 80 -

Haut. de la perpendiculaire......... 45 -

Dist. de cette perpend, au bord antér... 28 - 
dist., du même point de cette perpend.

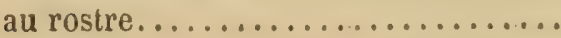

71 mill.

- enfin, de la base de la perpend. à l'angle postéro-dorsal...............

$53-$

La hauteur maximum est de 45 comme celle de la perpendiculaire, bien que cette hauteur maximun se trouve à 38 millimètres en arrière, juste à l'extrémité du ligament postérieur à l'endroit de l'angle postéro-dor'sal. Il résulte de là que les bords supérieur et inférieur sont parallèles sur une étendue de 38 mill.

On remarquera, en outre, que cette hauteur (45) maximun atteint presque la moitié de la longueur (98 mill.), et, que la convexité (39 mill.) égale pour ainsi dire, moins 6 millimètres, la hauteur, et dépasse le tiers de la longueur (49 contre 98); enfin, que cette convexilé est presque centrale.

D'après ces mesures, l'on peut se faire une idée de cette Anodonte, en se la représentant allongée, à bords parallèles, d'une élévation plus que médiocre et presque aussi ventrue qu'elle est haute. Cette forme très remarquable, ne peut être confondue avec aucune autre du système européen, même avec la Cyrtoptychia (gibba de Clessin) qui a sa partie postérieure un peu plus dilatée que l'antérieure.

Coq. de petite taille (98 de $\operatorname{long}$ sur 45 de haut), de forme allongée dans une direction légèrement descendante, à bords parallèles, très convexe, presque aussi ventrue que haute. Valves minces, faiblement bâillantes : $1^{\circ}$ à la région antéro-palléale; $2^{\circ}$ la partie postérieure, de l'angle postéro-dorsal au rostre.

Bord supérieur rectiligne-descendant. Région antérieure arrondie, peu développée, amoindrie vers son contour 
supéro-dorsal. Bord inférieur d'abord faiblement arqqué en avant, puis rectiligne, descendant comme le supérieur, enfin légèrement remontant vers le rostre. Région postérieure allongée, plus de deux fois plus longue ( 71 mill.) que l'antérieure (28 mill.), se terminant par un rostre assez prononcé, dont l'extrémité, sans être recourbée, regarde en bas. Entre ce rostre et l'angle postér o dorsal, les valves paraissent comme tronquées et le contour est brusquement recto-descendant avec une légère convexité près du rostr e.

Arête dorsale ventrue, très convexe, fortement accentuée (sans sillons anguleux), offrant une pente descendante très rapide sur la crête, qui, bien que peu développée, présente à l'extrémité du ligament une petite section comprimée, section se terminant à l'angle postéro-dorsal d'une façon amincie.

Sommets très gonflés, ventrus, pruéminents, sillonnés de rides émousées faiblement tremblotées.

Stries d'accroissement çà et là grossières, saillantes, par. fois liratiformes, représentant parfaitement la ligne des contours.

Epiderme peu brillant, d'une teinte sombre fauve-rou geâtre sur la région ombonale, plus jaunacée vers celle du bord palléal. Intérieur d'une nacre irisée-bleuâtre.

Liggament antéro-interne très développé à son extrémité antérieure et occupant presque toute l'épaisseur de la char. nière. Ligament postérieur robuste, saillant, avec une lunule allongée à son extrémité. Charnière légèrement sinueuse avecune faible denticulation un peu en arrière des crochets. Impressions très superficielles.

Ancien cours de la Seine dans les prés de Verrières, à 10 kilomètres en amont de Troyes (Aube). 


\section{HO CYGNAANA}

Les Cygnœana sont des Anodontes peu ventrues, de forme oblonscue parfois très allongée; à sommets comprimés, non proéminents; à bords supérieur ou inférieur ordinairement parallèles ou un peu arqués; à rostre arrondi, très obtus, sauf chez l'anserirostris. Ces espèces sonı surtout caractérisées par leurs sommets très antérieurs et par l'énorme développement de leur partie postérieure comparée à l'antérieure. Chez ces Anodontes, la région postérieure atteint trois ou quatre fois (comme chez la Nansoutyana) l'étendue de la région antérieure, et le maximum de la convexité n'est guère que le tiers ou le quart de la longueur.

Les $C y g n c a n a$ forment un groupe bien naturel, dont les différentes formes ne se trouvent guère que dans les étangs, les marais ou les cour's d'eaux tranquilles et vaseuses.

\section{Anodonta arenaría}

Mya arenaria, Schröter, Flussconch. Thuring Vaš, p. 163, pl. II, f. 1, 1779. (Non, Mya arenaria de Linnæus.)

Cette espèce, très bien représentée dans l'ouvrage de Schröter, est une Anodonte oblongue-allongée. (Long. 14"3 à 163 mill.), à région postérieure très longue, plus développée en hauteur que l'antérieure et caractérisée par un bord inférieur sinué vers sa partie médiane. Cetle sinuosité se fait sentir jusque sur lo milieu de la convexité. Les 
bords, chez l'arenaria, ne sont pas parallèles, mais sont légèrement divergents par suite de la plus grande dilatalion de la région postérieure.

Celte espèce est voisine de la Cygncea, dont elle diffère notamment par sa forme plus allongée, relativement moins hauie; par sa région antérieure plus rétrécie; par son bord inférieur sinué.

Cette Anodonte habite dans divers étangs et marais du nord de l'Allemagne. En France, elle se trouve typique dans l'étang de la Bazouge près de Chéméré (Mayenne) et dans le lac de la Négresse près de Bayonue, dans les Basses-Pyrénées (A. Milne-Edwards).

\section{Anodonta cygnaea}

Nytilus cygnæus, Linnceus, Syst. nat. (Ed.X, 179S), p. 706, $\mathrm{n}^{\circ} 218$, et Hanlıy, ipsa conch., p. 144, 18כ5.

Le type de cette espèce, pour ainsi dire inconnue, est l'Anodonta cellensis de Rossmässler, représentée dans son i conographie (iV, 1836) à la figure 280.

C'est cette Anodonte que presque tous les auteurs ont regardé comme la cellensis de Schröter (Flussconch. 1779) qui, entre parenthèses, n'a jamais publié d'espèces sous ce nom. Il convient donc maintenant d'éliminer cette appellation de cellensis pour la remplacer par celle de судпæа.

Quant à cette même appellation de cygncea que l'on trouve dans la plupart des auteurs, elle doit, lorsqu'elle ne sert pas, comme cela arrive souvenl, de cache-ignorance pour désigner des séries d'espèces que l'on n’a pas su dé- 
terminer, elle doit, dis-je, être rapportée soit à la pammegala, soit à l'eucypha.

J'ai reçu la cygnææa type de Linnæus: $1^{\circ}$ Du Danemark sous le nom de cellensis type et de cellensis var. cariosa (voir Mörch, Syn. Moll. Daniæ, p. 84 et 85, 1864); - 2 de Manchester (Angleterre); - $3^{\circ}$ de Rosenbach entre Roth see et Ebikon en Suisse; - $4^{0}$ des environs de Gênes en Italie.

En France, je la connais des marais de Villechetif près de Troyes, dans l'Aube (J. Ray); de la Grosne, dans Saône-et-Loire (Locard); des fossés du Rhòne à Lyon, et, du Menthon dans l'Ain (Locard); du Rhône près d'Arles (Fagot); de l'Étang-Neuf près de Saint-Saulge dans la Nièvre (Brevière); du lac de la Négresse près de Bayonne (A. Milne-Edwards); enfin, du lac du Bourget, dans la Savoie.

\section{Anodonia anserlrostris}

Anodonla anserirostris, Kuster, anod. (in 2 édit. Chemnitz), p. 5 ๖ , pl. X, f. 3 et pl. XI*, fig. 1-2, 1852. (Ne pas confondre avec les figures 1-2 de la pl. XI qui représentent la tumida).

Espèce caractérisée par un rostre bien accentué, légèrement ascendant et imitant un peu celui de l'Helvetica. Les figures données par Kuster suffisent pour la connaissance de cette Anodonte.

Je l'ai reçue de Fredericksberghave dans le Danemark, sous le nom de Cellensis var. sulcata (Mörch, Syn. Moll. Daniæ, p. 85̆, 1864).

Le type a été recueilli en Bavière dans l'Altmühl, petite 
rivière qui se jette dans le Danube, près de Ratisbonne.Je ne la connais pas de France.

\section{Anodonta Saint-Simoniama.}

Anodonta Saint-Simoniana, Fagot, in Litt. 1881.

Jolie espèce peu convexe, oblongue, en forme de fer de lance, à contours sans parties anguleuses (angle postérodorsal tellement émoussé qu'il n'est pas sensible), caractérisée par une région postérieure près de trois fois plus longue que l'antérieure, régulièrement dilatée à sa partie moyenne, avec une extrémité rostrale comprimée, si largement arrondie que le rostre n'est pas défini. Cette Anodonte, au point de vue de la forme, est pour le groupe de la Cygncea ce qu'est la Rayi pour celui de l'analina.

Long. $\max . . . . \ldots \ldots \ldots \ldots \ldots . . . . . . .107$ mill.

Haut. $\max . \ldots \ldots \ldots \ldots \ldots \ldots \ldots$. 6 -

Épaiss. max (à 30 des sommets; - à 57

$1 / 2$ du rostre; - à 49 du bord antér.;

- à 27 de l'angle postéro-dorsal; -

à 32 de la base de la perpendiculaire.)

Long. de la crête ligamento-dorsale, des sommets à l'angle postéro-dorsa'.... 41 -

Dist. de cet angle au rostre......... 50 -

Corde apico-rostrale............. 86 -

Haut. de la perpendiculaire......... 49 -

Dist. de cette perpend. au bord antér... 29 -

- du même point de cette perpend. au

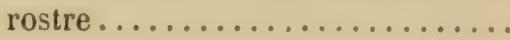

- enfin, de la base de la perpendiculaire à l'angle postéro-dorsal...... 
Coq. en forme de fer de lance, oblongue-allongée dans une direction légèrement descendante, à summets très antërieurs, à valves comprimées peu convexes, presque aplaties vers l'extrémité postérieure; enfin, non bâillantes, sauf très faiblement en dessous de l'angle postérodorsal, qui est si émoussé que la courbe du bord supérieur n'est point interrompue.

Bords supérieur et inférieur offrant tous les deux une convexité semblable, régulière depuis l'extrémitẻ antérieure jusqu'à la postérieure. Région antérieure peu développée, comme amoindrie, cependant bien ronde. Région postérieure largement dilatée (surtout au niveau de l'extrémité du ligament), près de trois fois plus longue que l'antérieure, et se terminant par un large rostre comprimé, dont le contour arrondi ne donne lieu à aucune saillie anguleuse. Au-dessus de cette région rostrale, le contour se poursuit bien arqué jusqu'à l'angle postérodorsal, qui est nul et dont on ne saisit l'emplacement qu'en vérifiant l'endroit de la lunule terminale du ligament.

Sommets non proéminents, comme aplatis (crochets très aigus), sillonnés de rides concentriques très émoussées et légèrement tremblotées.

Arc̀te dorsale nulle, indiquée seulement par une radiation zonulée d'un noir verdâtre. Grête ligamento-dorsale non saillante, assez comprimée à son extrémité.

Stries d'accroissement fines, régulières, plus fortes et parfois assez saillantes vers les contours, enfin, paraissant feuilletées vers les régions postérieure et palléale.

Épiderme brillant, d'un faure-cendré, passant postérieurement au fauve-brique, et prenant, vers les contours, une nuance verdâtre plus ou moins sombre. Intérieur d'une belle nacre irisée bleuâtre. 
Ligament antéro-interne filiforme, occupant toute l'épaisseur de la région cardinale. Ligament postérieur trìs allongé, peu saillant, ordinairement symphynoté, terminé par une petite lunule étroite-allongée. Charnière presque nulle, seulement un tant soit peu épaissie sur la région latérale. Impressions très superficielles.

Cette Anodonte, dédiée à $M$. Alfred de Saint-Simon, a été recueillie dans le canal de Carcassonne dans l'Aude (Fagot), ainsi que dans le lac de Neuchâtel, près de la ville de ce même nom, en Suisse (Coutagne).

\section{Anoilonta Fagoti.}

Long. $\max . . . \ldots \ldots \ldots \ldots \ldots \ldots, 100$ mill.

Haut. $\max . . . \ldots \ldots \ldots \ldots \ldots \ldots$. 50 -

Épaiss. max (à $\check{~} 9$ des sommets; à 54 du rostre; à 45 du bord antér.; à 27 de l'angle postéro-dorsal ; à 31 de la base de la perpend.)................ mets à l'angle postéro-dorsal.

Distance de cet angle au rostre........

Corde apico-rostrale..............

IIaut. de la perpendiculaire..........

Dist. de cette perpend. au bord antér...

- du même point de cette perpend. au rostre.....................

- enfin, de la base de la perpend. à l'angle postéro-dorsal........... 57 -

D'après ces mesures, l'on voit que la Fagoti est une espèce oblongue très allongée, à région postérieure ( 75 mill.) 
trois fois plus longue que l'antérieure (25 mill.); par conséquent à sommet très en avant. Sa hauteur maximum, qui se trouve à 30 mill. en arrière de la perpendiculaire, est juste moitié ( 50 mill.) de la longueur (100 mill.); entỉn sa convexité est un peu plus du quart de la longueur.

Bord supérieur presque rectiligne. Bord inférieur légèrement arqué, avec son maximum de convexité à 30 mill. en arrière de la perpendiculaire. Région antérieure médiocre, régulièrement arrondie. Région postérieure très allongée (trois fois plus que l'antérieure), faiblement dilatée vers sa partie moyenne, puis allant en se rétrécissant par un contour arqué jusqu'à un rostre très obtus, bien arrondi, non anguleux, regardant bien en direction rectohorizontale. Contour supérieur, de l'angle postéro-dorsal au rostre, rectiligne-descendant, s'arrondissant vers le rostre.

Valves peu épaisses, non ou à peine bâillantes entre le rostre et l'angle postéro-dorsal. Arîte dorsale non saillante, confondue dans la convexité et offrant entre elle et la crête ligamento-dorsale, ainsi que vers le rostre, une pente douce et régulière.

Sommets arrondis, non proéminents, sillonnés de riles concentriques très émoussées, à peine tremblotées.

Stries d'accroissement saillantes et feuilletées seulement vers la ligne des contours, notamment sur la partie postérieure.

Épiderme feu brillant, d'une couleur fauve-olivâtre, plus claire sur la partie ventrale et sillonnée, en outre, par des radiations vertes peu prononcées. Intérieur d'une nacre blanche-bleuacée.

Ligament antérieur feuilleté, filiforme, occupant toute la charnière jusqu'à 10 mill. en arrière des crochets. LiY'AI. 1881. 
gament postérieur robuste, proéminent, relativement peu allongé et terminé par une lamelle étroite trìs longue (12 mill.). Charnière sans particularité, seulement un peu épaissie à la région latérale.

Cette Anodonte, que je dédie au malacologiste Paul Fagot, habite dans le canal du Midi, entre Toulouse et Villefranche-de-Lauraguais (Haute-Garonne).

\section{Anodonta oblonga.}

Anodonta oblonga, Hillet, Desc. deux nouv. esp. Anod. in: Mém. Soc. agric. sc. Angers. 1. (3e livr. 1833), p. 242, pl. 12, f. 1 .

Cette espèce, bien représentée par Millet, a été également figurée d'une façon exacte, par Dupuy (Hist. moll. France, $-6^{\text {e }}$ fasc. 1852 , pl. XVIII, f. 13), et par Joannis (Et. Nayades, in : Ann. soc, Linn. de Maine-et-Loire, IIl, 18ə̃8, pl. 4, f. 1), sous l'appellation erronée de Cygnoea. var. intermedia de Lamark. Je donnerai plus tard les caractères de l'intermedia, qui est une forme différente de celle.ci.

L'Oblonga est une Anodonte très abondante dans toutes les rivières tranquilles ou vaseuses (Maine, Mayenne, le Couasnon) du département de Hainc-et-Loire.

Je la connais des environs de Rennes, dans l'llle-et. Vilaine (1.etourneux); des étangs de Saint-Hubert, près de Rambouillet et de Trappes dans Seine-et-Oise (Mabille); de la Ilasle, près d'Amboise (Rambur); de la Bonde-Gendret à Troyes, dans l'Aube; d'un bassin marécageux à SaintLaurent-de-L'Ain, vis à-vis Mâcon (Locard); du lac du 
Bourget, en Savoie; du canal de Bouc à Arles, etc. M. Coutagne a recueilli cette espèce en Suisse dans le lac de Neuchâtel, près de la ville du même nom.

\section{Anodonta condatina.}

Anodonta condatina, Letourneux, in Litt., 1880.

Cette nouvelle espèce diffère de l'oblonga, dont elle est voisine, par une convexité plus forte, régulièrement bombée et renflée dans toutes ses parties, et dont le maximum du renflement se trouve bien médian. Chez l'oblonga, la convexité n'est pas régulière. Les rẻgions antérieure et palléale sont ordinairement comprimées. La postérieure notamment, s'atténue assez régulièrement. Le maximum de la convexité est tantôt assez rapproché des sommets, tantôt assez éloigné. Chez la condatina, des sommets au bord palléal et de l'extrémité antérieure au rostre, les valves sont uniformément renflées-ventrues et offrent une convexité bien régulière.

Cette Anodonte, d'une belle couleur verte ou olivâtre uniforme, habite à Moulin-le-Comte, près de Rennes, dans l'Ille-et-Vilaine (Letourneux). Elle vit également dans le lac d'Aiguebelle, en Savoie (Coutagne).

\section{Anodonta carionsa.}

Anodonta cariosa, Küster, gatt. Anodonta in : Chemnitz, $2^{\circ}$ édit., p. 43, pl. IV, fig. 3 (sous le nom de Cellensis, et pl. V, f. 1, et pl. X, f-1-2, 1852.

Anodonta cellensis, var. rostrata, Brot, Et. Nayades, p. 37, pl. IV, f. 1, 1867. 
Abondante dans le Regnitz près d'Erlangen, en Bavière. Etang dans la campagne de l'Elysée, à Celigny (Suisse). En France, elle se trouve bien typique dans l'Erve à Thévalles près de Chéméré (Mayenne).

La Cariosa est bien représentée dans les planches que je viens de citer. M. Drouët, dans ses Naiades, a confondu cette espèce avec l'oblonga de Millet.

\section{Anodonta Nanvoutyana.}

Cetie Anodonte, que je dédie au savant métérologiste Champion du Bois, comte de Nansouty, a été trouvée dans le lac de la Négresse près de Bayonne, dans les BassesPyrénées (A. Milne-Edwards).

Cette espèce de la taille et de la forme de la cariosa est caractérisée par une partie antérieure réduite presque à rien, par suite des sommets qui sont tout à fait en avant.

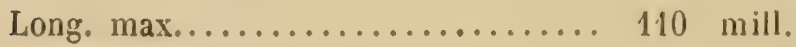

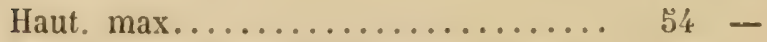

Epaiss. max. (à 34 des sommets; à 61 du rostre; à 50 du bord antérieur; à 30 de l'angle postéro-dorsal; à 37 de la base de la perpendiculaire.)........

Long. de la crête ligam. dors., des sommets à l'angle postéro-dorsal....... 52 -

Dist. de cet angle au rostre......... 45 -

Corde apico-rostrale............. 93 -

Haut. de la perpendiculaire........ 47 -

Dist. de cette perpend, au bord antér... 22 -

- du même point de cette perpend. au rostre................ 88 - 
Dist., enfin, de la base de la perpend. à l’angle postéro-dorsal........... 67 mill.

On remarque, d'après ces mesures, $1^{\circ}$ que la région postérieure est quatre fois plus longue que l'antérieure qui n'a que 22 millimètres; $2^{\circ}$ que la crête ligamentodorsale est très allongée, puisqu'elle a 52 contre 45 , de l'angle postéro-dorsal au rostre. Chez presque toutes les Anodontes, la partie intermédiaire entre l'angle postérodorsal et le rostre est toujours plus grande, ou, si elle n'est pas plus grande, elle est au moins égale ou presque égale à la partie dorsale qui se prolonge des sommets à l'ar,gle postéro-dorsal. Ainsi, par exemple:

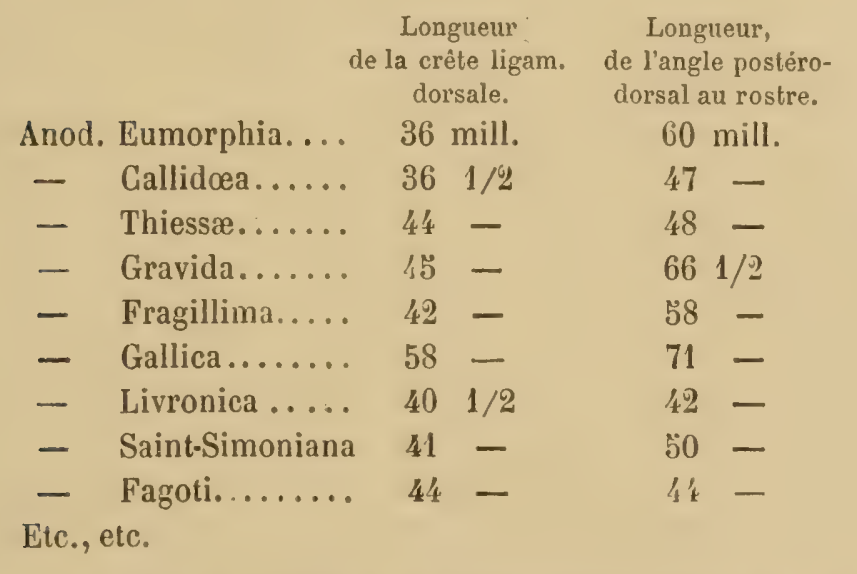

Lietle grande longueur de la crête dorsale résulte de la position des sommets presque tout à fait antérieure; aussi, la région antérieure est-elle excessivement exiguë. Chez toutes les autres Anodontes, la région postérieure atteint le double ou bien trois fois la longueur de l'anté- 
rieure; mais la Nansoutyana est, à ma connaissance, la seule espèce (sauf la Ressmanni) où elle atteint quatre fois l'étendue de la partie antérieure.

La plus grande hauteur ( 54 mill.), chez cette espèce, se trouve située à 35 mill. en arrière de la perpendiculaire, qui n'a que 47. Quant à la convexité peu prononcée $(29$ mill.) elle offre son maximum presque à égale distance des sommets, de l'angle postéro-dorsal et de la base de la perpendiculaire, tout en étant un peu plus rapprochée du bord antérieur que du rostre.

Coq. oblongue-allongée, à sommets très antérieurs, à valves asssez minces, toujours encrassées de calcaire et non bâillantes, sauf au bord antéro-palléal, où le bâillement se trouve réduit à une toute petite fente.

Bord supérieur d'abord rectiligne, puis convexe-descendant sur le rostre. Région antérieure très exiguë, arrondie. Bord inférieur faiblement convexe, puis remontant à son extrémité rostrale. Région postérieure excessivement longue (quatre fois plus que l'antérieure), régulièrement atténuée, terminée par un rostre arrondi, très émoussé, regardant en avant. Sommets aplatis, non proéminents, toujours excoriés, et, malgré l'excoriation, laissant voir des rides concentriques étroites, assez rapprochées.

Arête dorsale régulièrement convexe, confondue dans la convexité générale de la région postérieure. Crête dorsale non ailée, mais assez comprimée. Stries d'accroissement émoussées, plus ou moins saillantes vers les contours et feuilletées à la région supéro-rostrale. Epiderme terne, d'une couleur sombre, d'un noir rougeâtre. Intérieur d'une nacre bleuacée irisée. Ligament antéro-interne filiforme, occupant toute la région de la cardinale et se 
prolongeant à 11 mill. en arrière des crochets. Ligament postérieur peu saillant, peu allongé (27 mill.) et se terminant par une très longue lunule (23 mill.) très étroite. Charnière nulle, impressions : postérieure superficielle; antérieure assez profonde en forme de palette.

\section{Anodonta Boulinsiana.}

Anödonta rostrata, Dupuy, Gat. extram. Galliæ test. n॰ 27. 1849 (non Rossmässler).

Anodonta Noulinsiana, Dupuy, moll. France, 6 e fasc., 1839, p. $616, \mathrm{pl} . \mathrm{XX}, \mathrm{f} .19$.

Cette espèce, très voisine de la cariosa, dont elle pourrait à la rigueur être considérée comme une forme minor, possède, comme celle-ci, des sommets très antéricurs. Elle parait particulière aux eaux vaseuses des étangs du littoral aquitanique, où elle vit à Cazeaux, à Aureillan dans les Landes.

Il existe dans les Suites à Rossmässler (fig. 165๊) une soidisant Moulinsiana qui ne ressemble pas du tout à l'espèce de l'abbé Dupuy. Cette forme, qui doit plutôt se rapporter à l'Anodonta lacuum, diffère de la Moulinsiana: par son bord inférieur arqué, se relevant à l'extrémité rostrale (chez la Moulinsiana le bord est rectiligne jusqu'à l'extrémité); par sa crête dorsale comprimée, un peu ailée, carrément anguleuse à son extrémité et descendant sur le rostre sous l'apparence d'un contour concave (chez la Moulinsiana, la crête, ni comprimée, ni ailée, est à peineanguleuse à l'extrémité du ligament; le contour depuis le sommet jusqu'au rostre est presque régulièrement hombé); 
par son arête dorsale très émoussée, un tant soit peu concave (celle de la Moulinsiana, légèrement bianguleuse, descend en ligne droite sur un rostre carrément coupé et non arrondi-remontant comme celui de cette soi-disant Moulinsiana.) J'ajouterai encore que l'espèce de l'abbé Dupuy est une forme plus allongée, moins haute, d'une convexité différente, etc.

Les Suites à Rossmässler, Suites vraiment déplorables au point de vue scientifique, sont pleines de ces sortes d'erreur de détermination.

\section{Anodonta Ranarum.}

Anodonta Ranarum, Morelet, Moll. Portug., p. 10\%. pl. XII, f. 2,1845 .

Cette espèce, très bien représentée dans l'ouvrage du Portugal, et, qui n'a pas le moindre rapport avec l'anatina, quoiqu'en dise l'auteur, mais qui présente tous les caractères des Cygnceana, habite en Portugal, dans les aflluents dela Guadiana qui descendent des hautes vallées de l'Algarve, entre Mertola et Castro-Verde.

\section{Anodonta Rhodani.}

Long. max................... $761 / 2$

Haut. $\max . . . \ldots \ldots \ldots \ldots \ldots . .6 . .45$ mill.

Epaiss. max. (à 20 des sommets; à 43 du rostre; à 34 du bord antérieur; à 21 de l'angle postéro-dorsal; à 29 de la base de la perpend.)........... $22-$ 
Long. de la crête ligam.-dor's, des sommets à l'angle postéro-dorsal........

Dist. de cet angle au rostre......... 37 -

Corde apico rostrale............ 61 1/2

Haut. de la perpendiculaire.......... 41 -

Dist. de cette perpend. au bord antér... 22 -

— du même point de cette perpend. au rostre..................... 54

- enfin, de la base de la perpend, à l'angle postéro-dorsal............. 49 -

Cette espèce très comprimée, puisque sa convexité (22 mill.) est près de trois fois et demie moindre que sa longueur (76 1/2 mill.), est une Anodonte de forme oblongue.ovoïde, relativement haute( 45 mill.) pour sa longueur, et dont les contours ne présentent aucune partie anguleuse.

Valves minces, baillantes seulement au bord antéropalléal.

Bord supérieur assez fortement convexe. Région antérieure arrondie. Bord inférieur un peu moins convexe que le supérieur. Région postérieure près de deux fois et demi plus développée que l'antérieure, se terminant par une partie rostrale large, à contour arrondi.

Arête dorsale non prononcée, se confondant dans la convexité et offrant une pente régulière sur la crête dorsale peu accentuée, non ailée ni comprimée, dont l'angle postéro-dorsal est fort émoussé.

Sommets comme écrasés, presque plans (crochets très aigus), sillonnés par de petites rides concentriques peu distantes les unes des autres et assez saillantes.

Stries d'accroissement fortes, grossières, souvent irrégu- 


\section{$-154-$}

lières, rendant la surface des valves comme rugueuse.

Epiderme d'un ton sale, terne, d'une teinte uniforme d'un jaune-terreux ou d'un cendré-olivâtre, passant au noir vers les contours. Intérieur d'une nacre irisée de tons jaunacés-verdâtres d'une nuance livide.

Ligamenl antéro-interne court, se confondant avec le postérieur qui est fort, saillant et relativement allongé. Région cardinale de la charnière nulle. Rérgion latérale assez épaisse. Impressions, superficielles, mal définies.

Ceite Anodonte vit dans le canal de Bouc à Arles. Je la connais encore du Morin à Esbly et des étangs de Trappes dans Seine-et-Oise. (Mabille).

\section{Anodonia Arverniea.}

Long. $\max . . . \ldots \ldots \ldots \ldots . . .6 .61$ mill.

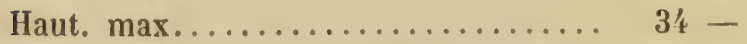

Epaiss. max. (à 16 des sommets; à 35 du rostre; à $27 \mathrm{du}$ bord antér.; à $17 \mathrm{de}$ l'angle postéro-rostral; à 21 de la base de la perpend.).............. 18 -

Long. de la crête ligam.-dors. des sommets à l'angle postéro-dorsal......... 27 -

Dist. de cet angle au rostre......... 27 -

Corde apico-rostrale............. 50 -

Haut. de la perpendiculaire......... 28 -

Distance de cette perpend. au bord antér. 16 -

— du même point de cette perpend. au rostre................... 43 -

- enfin, de la base de la perpend. à l'angle postéro-dorsal........... 37 - 
Chez cette Anodonte, la hauteur maximum se trouve à 26 millimètres en arrière de la perpendiculaire; la partie antérieure est presque trois fois plus petite que la postérieure; la crête ligamento-dorsale (27 mill.) est égale à la distance de l'angle postéro-dorsal au rostre.

Cette espèce est une coquille allongée, d'une apparence spathuliforme, sans parties anguleuses sur son contour, et à valves relativement assez fortes, très bâillantes à sa région antéro-palléale.

Bord supérieur subrectiligne ou un tant soit peu.convexe jusqu'à l'angle postéro-dorsal, qui est très émoussé, puis à partir de cet angle descendant presque en droite ligne sur le rostre. Région antérieure exiguë, arrondie, décurrente à sa base. Bord inférieur descendant, d'abord légèrement convexe, puis très faiblement sinué à 7 ou 8 millimètres en arrière de la perpendiculaire ; enfin, devenant notablement convexe à son extrémité, par'suite d'une dilatation des valves en cette partie. Région pustérieure allongée dans une direction faiblement descendante, se dilatant au bord postéro-palléal et se terminant par un large rostre très obtus.

Arête dorsale confondue dans la convexilé, descendant en pente régulière sur la crête dorsale, qui est peu accentuée. Sommets comprimés, néanmoins faiblement ventrus-proéminents, toujours excoriẻs.

Stries fines, non saillantes, sauf vers les contours, où elles deviennent un peu plus fortes et un tant soit peu feuilletées.Épiderme d'une nuance sombre avec des zones concentriques plus claires d'un cendré-jaunacé plus ou moins olivâtre. Intérieur d'une belle nacre épaisse d'un blanc-bleuacé et paraissant entièrement et très finement chagrinée. 
Ligament antéro-interne occupant toute l'épaisscur de la charnière, se prolongeant à 5 ou 6 millimètres en arrière des crochets. Ligament postérieur robuste, à demi symphynoté, terminé par une lunule allongée.

Charnière presque nulle, à région cardinale un peu ondulée, par suite de l'envahissement des attaches du ligagament antérieur. Impression antérieure plus prononcée que la postérieure qui est très superficielle.

Cette Anodonte vit en Auvergne, d'où je l'ai reçue sans indiçation précice de localité, sous le nom erroné de palustris de d'Orbigny. La vraie palustris est une forme toute différente du groupe de l'anatina.

\section{ELITIPSORAIANA}

Les espèces de ce groupe, comme l'indique son nom, sont des Anodontes caractérisées par une croissance ellipsoïde et par des stries d'une grande excentricité sur la région pustérieure. Chez ces $A$ nodontes, les sommets sont très en avant et la région antérieure est exiguë, surtout très peu haute, par suite de la partie inféro-palléale qui est fortement décurrente.

Les Eliipsopsiana vivent indifféremment dans les rivières, les lacs ou les étangs. Elles ont été rencontrées jusqu'à présent en Carynthie, en Bavière, en Suisse, dans le Jura français, ainsi qu'aux environs de Paris et de Troyes (Aube).

\section{Anodonta cllipsopsis.}

Long. $\max . \ldots \ldots \ldots \ldots \ldots \ldots . . .67$ mill.

Haut. $\max \ldots \ldots \ldots \ldots \ldots \ldots \ldots . .62-$ 
Épais. max (à 30 des sommets; à 46 du rostre; à 41 du bord antérieur, à 25 de l'angle postéro-dorsal; à 29 de la brse de la perpend.)............... 23 mill.

Long, de la crête ligam.-dors., des sommets à l'angle postéro-dorsal........... 32 -

Distance de cet angle au rostre........ 50 -

Corde apico-rostrale............. 74 -

Haut. de la perpendiculaire.......... $46 \quad 1 / 2$

Dist. de cette perpend. au bord antér... 23 -

— du même point de cette perpend. au rostre................... 65 -

- enfin, de la base de cette perpend. à l'angle postéro-dorsal.......... รัз -

Cette espèce, à stries ellipsoïdes, est une forme oblongue, peu allongée, relativement très haute (əั2 mill.) pour sa longueur ( 87 mill.) et très comprimée (23 mill.). Sa convexité bien régulière, presque médiane, s'atténue d'une façon égale sur ses contours, qui sont sans parties anguleuses.

Cor. de forme oblongue dans une direction légèrement descendante, à valves comprimées, minces, à peine bâil . lantes en avant et au-dessus du rostre, à surface externe rugueuse grossièrement striée, d'une teinte uniforme foncée, presque toujours recouverte d'un enduit limoneux.

Bord supérieur presque rectiligne ou bien légèrement arqué du bord antérieur jusqu'à l'extrémité du ligament, puis descendant assez brusquement sur le rostre. Région antérieure peu développée, peu haute, amoindrie notamment vers la partie inférieure par suite de la direction ellipsoïde des stries, de telle sorte que la rotondité maxi- 
mum du contour s'accentue surtout vers le bord antérosupérieur. Bord inférieur rógulièrement convexe-arrondi depuis le commencement supérieur de la région antérieure juqu'au rostre. Région postérieure près de trois fois plus longue que l'antérieure, large, bien développée en hauteur jusqu'à l'extrémité du ligament, puis diminuant et se terminant en un rostre très obtus.

Arête dorsale nulle, confondue dans la convexité des valves ou bien se présentant, sur quelques échantillons (provenant du lac des Quatre.Gantons), plus accentuée avec une pente plus rapide sur la crête dorsale, qui, dans ce cas, est bien plus amoindrie que celle des échantillons où l'arête dorsale est nulle.

Sommets tout à fait aplatis, presque toujours excoriés, très rarement en bon état, offrant alors des rides tremblotćes relatirement saillantes et robustes, sur lesquelles on remarque une suite de petites éminences tuberculeuses disposées sur la ligne d'origine de l'arête dorsale.

Stries d'accroissement en ellipses, rugueuses, grossières, saillantes et un peu feuilletées vers les contours. Epiderme terne, d'un ton jaunacé, rougeâtre ou bien livide sur la partie ventrale, puis passant vers les contours à une nuance très foncée tirant sur le noir. Intérieur d'une nacre bleuâtre assez prononcée.

Ligament antéro-interne filiforme se prolongeant très loin en arrière des crochets, qui offrent comme une petite partie calyculée. Ligament postérieur court, fort, saillant, terminé par une grande lunule triangulaire. Charnière complètement nulle. Impressions superficielles.

Le type vit dans les marais ou les flaques d'eaux entre le Rhône et la Cascade de Pissevache, près de Vernayaz, dans le Valais, en Suisse. On rencontre encore cette es- 
pèce dans le lac des Quatre-Cantons, près de Küssnacht.

J'ai trouvé aux environs de Troyes (Aube), dans un bras de la Seine, une charmante variéte minor de cette Anodonte. Cette variété tout à fait semblable, par ses caractères, au type de Suisse mérite d'être signalée à cause de sa taille exiguë.

Long. $\max . \ldots \ldots \ldots \ldots \ldots \ldots \ldots . \ldots . . . .53$ mill.

Haut. $\max . \ldots \ldots \ldots \ldots \ldots \ldots \ldots \ldots \ldots . \ldots . \ldots$

Épaiss. $\max . \ldots \ldots \ldots \ldots \ldots \ldots \ldots \ldots \ldots 16$ -

Long, de la crête ligam.-dorsale....... 20 -

Dist. de l'angle postéro-dorsal au rostre.. 29 -

Corde apico-rostrale.............. 45 -

Haut. de la perpendiculaire.......... 30 -

Dist. de cette perpend. au bord antér.... 1:3 -

- du même point de cette perpend. au rostre................... 38 -

- enfin, de la base de la perpend. à l'angle postéro-dorsal............. 32 -

\section{modonta Ressmanni.}

Long. $\max . . . \ldots \ldots \ldots \ldots . . . . .99$ mill.

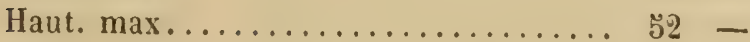

Épaiss. max. (à 19 des sommets; à 79 du rostre; à 33 du bord antér.; à 29 de l'angle postéro-dorsal et de la base de la perpend.)................. 27 -

Long. de la crête ligam.-dors., des sommets à l'angle postéro-dorsal........ 41 -

Distance de cet angle au rostre........ 53 - 
Gorde apico-rostrale............. 88 mill.

Haut. de la perpendiculaire......... 39 -

Dist. de cette perpend. au bord antér... 18 -

- du même point de cette perpend. au rostre.................. 81 -

- enfin, de la base de la perpend. à l'an. gle postéro-dorsal............ 54 -

Chez cette singulière espèce, à stries ellipsoïdes, on remarque d'après ces mesures : $1^{\circ}$ Que la région antérieure (18 mill.) est, à un millimètre près, cing fois plus courte que la postérieure (81 mill.); c'est avec la Nansoutyana, l'Anodonte qui offre une aussi grande différence de taille entre les deux régions antérieure el postérieure; $2^{\circ}$ que la convexité (27 mill.) est plus de trois fois moindre qque la longueur; $3^{\circ}$ que cette convexité, à égale distance de l'angle postéro-dorsal et de la base de la perpendiculaire, a son maximum de renflement très rapproché des sommets, à 19 mill. seulement, tandis qu'il est éloigné de l'extrémité rostrale de 79 mill.; $4^{\circ}$ enfin, que la hauteur maximum (52 mill.), située à 36 millimètres en arrière de la perpendiculaire, dépasse notablement la hauteur de cette ligne (39 mill.). Il résulte de ce dernier caractère que cette Anodonte augmentant en hauteur jusqu'à 36 de la perpendiculaire, pour diminuer ensuite vers l'extrémité rostrale, est oblongue-allongée en forme de fer de lance.

Coq. à valves minces, assez bâillantes au contour antéro-palléal et postérieurement entre le rostre et l'angle postéro-dorsal.

Bord supérieur faiblement arqué à la région des sommets, puis fortement convexe-descendant postérieurement. Région antérieure très exiguë, arrondie-décurrente à son 
contour inférieur, où elle parait fort écourtée. Bord inférieur convexe-descendant jusqu'à ses deux tiers postérieurs, puis remontant vers le rostre. Région postérieure très développée, allongée en forme de fer de lance, allant en s'atténuant régulièrement à partir du point maximum du renflement pour devenir vers les contours très aiguễcomprimée; enfin, terminée par une extrémité rostrale aplatie, très obtuse-arrondie.

Arête dorsale très convexe (seulement à la région ombonale), offrant une pente presque brusquement descendante sur la crête ligamento-dorsale, qui, sans être proéminente, se comprime vers son extrémité postérieure. Sommets renflés, ventrus, assez proéminents, toujours excoriés.

Stries de forme ellipsoïde, assez fines sur la convexité ombonale, devenant ensuite vers les contours et sur toute la région postérieure (toujours encrassée de calcaire), saillantes, grossières et feuilletées.

Epiderme peu brillant, d'une teinte foncée grisâtre en avant, rougeâtre sur la région postéro-ombonale, enfin, jaunacée-terreuse passant au marron vers l'extrémité postérieure. Intérieur d'une nacre bleuacée-noirâtre.

ligament antéro-interne, filiforme, très court (19 mill.), s'étendant autant en arrière qu'en avant des crochets. Ligament postérieur allongé, saillant, aux trois quarts symphynoté, terminé par une grande lunule triangulaire. Charnière nulle, sauf vers l'extrémité postérieure où l'on remarque une dilatation plane assez large. Impressions superficielles; l'antérieure est parfois asse'z accentuée.

Cette belle Anodonte que je dédie au $\mathrm{D}^{\text {s }}$ Ressmann, a eté recueillie par ce savant dans ses viviers de Malborgeth en Carynthie.

MAI. 1881. 


\section{Anodonta slliqua.}

Anodonta siliqua, Küster, G. Anod. (in $2^{2}$ édit. Chemnitz), p. 57, pl. XIV, f. $5,1852$.

Cette belle espèce, dont Küster ignorait la provenance, a été parfaitement représentée par cet auteur, d'après des échantillons du musée d'Erlangen, en Bavière.

La siliqua vit dans la rivière bavaroise le Regnitz. Je la connais parfaitement typique, sauf une taille un peu plus grande, de la mare de Tourmont près de Poligny, dans le Jura (Mabille.)

Anodonta Tritonum.

Anodonta Tritonum, Coutagne, mss.

Long. $\max$

74 mill.

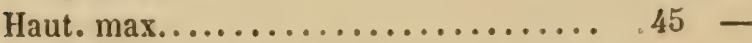

Epaiss. max. (à 20 des sommets, à 43 du rostre, à 32 du bord antérieur, à 16 de l'angle postéro-dorsal, à 24 de la base

de la perpend.)..................
Lung. de la crête ligam-dors. des sommets à l'angle postéro-dorsal.......... 21 -

Dist. de cet angle au rostre.......... 47 -

Corde apico-rostrale............. 64 -

Haut. de la perpendiculaire......... 37 -

Dist. de cette perpend. au bord antér... 18 - 
Dist. du même point de cette perpend. au rostre..................... 56 mill.

- enfin, de la base de la perpend.à l'an. gle postéro-dorsal.............. 41 -

La longueur de la crête ıgamento-dorsale, d'après cette mensuration, est remarquablement courte ( 21 mill.), en comparaison de la distance de l'angle postéro-dorsal au rostre (47 mill.)

La tritonum a une forme oblongue-ovoïde dans une direction oblique-descendante à partir des sommets; ses valves d'une teinte sombre, sillonnées de -stries rugueuses et grossières, n'offrent qu'un seul bâillement, qui s'étend sur tout le contour antéro et inféro-palléal.

Bord supérieur très court, presque rectiligne. Région antérieure arrondie, exiguë, décurrente à la base. Bord inférieur descendant, légèrement convexe, puis remontant, en s'arrondisant à son extremité. Région postérieure plus de trois fois plus longue que l'antérieure, très allongée dans une direction obliquement descendante, terminée par une partie rostrale assez comprimée, obtuse et arrondie, offr'ant, en outre, entre l'angle postéro-dorsal et le rostre, un contour très incliné, rectiligne ou un tant soit peu convexe.

Sommets très obtus, non proéminents, comme aplatis, fortement sillonnés de rides sinuées et possédant des crochets saillants, très aigus. Arête dorsale se confondant dans la convexité. Crête dorsale très exiguē, assez comprimée vers l'angle postéro-dorsal, qui est des plus obtus.

Stries rugueuses, grossières, surtout vers les contours. Epiderme d'une teinte uniforme brune-noirâtre, presque 
toujours recouverte d'un enduit noir très persistant. Intérieur d'une belle nacre bleuâtre.

Ligament antéro-interne lamelleux, occupant presque toute l'épaisseur de la cardinale qui est plus ou moins sinuée et parfois unituberculeuse. Ligament postérieur fort, très épais et saillant, terminé par une lunule triangulaire. Impressions profondes; la postérieure spathuliforme.

La tritonum a été découverte par l'ingénieur et savant malacologiste G. Coutagne, dans l'Yvette près Orsay,(Seineet-Oise.)

\section{Anodonta Antorida}

Cetteespèce est une des plus charmantes petites Anodontes que je connaisse. C'est une miniature de la stataria de Ray, seulement à stries ellipsoïdes. La stataria a 140 à 155 de longueur sur une hauteur de 70 a 75 et une épaisseur de 45 à 48 millimètres. L'antorida n'a que 56 de long, sur 31 de haut et 18 d'épaisseur. On voit quelle grande différence de taille et de grosseur il y a entre ces deux espèces.

Voici, du reste, la mensuration exacte de l'antorida.

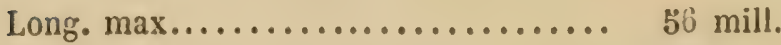

Haut. $\max . . . . . . . . . . . . . . . . . .31$ -

Epaiss, max, (à 14 des sommets; à 34 du rostre; à 22 du bórd antér.; à 14 de l'angle postéro-dorsal ; à 20 de la base de la perpendiculaire).

Long. de la crète ligam.-dorsale, des sommets à l'angle postéro-dorsal........ 
Distance de cetangle au rowpe........ 31 mill.

Corde apico-rostrale............. 47 -

Haut. de la perpendiculaire.......... 28 -

Dist. de cette perpend. au bord antér... 14 -

- du même point de cette perpend. au rostre..................... 41 -

- enfin, de la base de la perpend. à l'angle postéro-dorsal............. 32 -

Coq. petite oblongue, médiocrement allongée, un peu en forme de fer de lance, à valves minces, bâillantes à la partie antéro-palléal.

Bord supérieur presque rectiligne jusqu'à l'extrémité du ligament, puis nettement recto-descendant sur la partie rostrale. Région antérieure exiguë, peu haute, cependant relativement moins décurrente et moins écourtée vers le bas que celle des autres espèces du même groupe. Bord inférieur arqué. Région postérieure près de trois fois plus longue que l'antérieure, terminée par un rostreobtus, coupé carrément à son extrẻmité.

Arête dorsale peu prononcée, présentant, malgré tout, deux lignes subanguleuses divergentes dans une direction légèrement coneave et allant des sommets aboutir aux parties supérieure et inférieure du rostre, où elles donnent lieu à deux angles très émoussés, qui limitent en haut et en bas la partie rostrale. Crête dorsale peu accentuce, non ailée, faiblement comprimée, sans angle postéro-dorsal bien défini.

Sommets très antérieurs, légèrement ventrus, excoriés et offrant, malgré l'excoriation, des rides concentriques relativement fortes assez ondulées.

Stries ellipsoïdes, peu prononcées sur la région ventrale, mais fortes assez rugueuses vers les contours. 
Epiderme d'une nuance foncée noirâtre, d'un ton rougeâtre vers la région des sommets. Nacre intéricure d'une teinte irisée bleuâtre devenant jaunacée livide sous les sommets.

Ligament antéro-interne filiforme. Ligarnent postérieur médiocre, ordinairement à moitié symphynoté, terminé par une lunule allongée-triangulaire. Charnière nulle. Impressions très superficielles, indécises.

L'antorida habite dans les cours d'eaux des environs de Marboz, dans le Jura.

\section{Go GrTciana}

Les Glyciana sont des espèces à test brillant (sauf la Lacuum), d'une taille médiocre, d'une forme oblongrueallongée, à contours anguleux (l'angle postéro-dorsal est surtout saillant et sensiblement comprimé); enfin, caractérisées par un mode de convexité diflicile à définir. Chez ces Anodontes, il existe à un degré plus ou moins prononcé suivant les espèces et même suivant les échantillons, comme des parties plus renflées ou plus déprimées les unes que les autres, sans que pour cela, ces parties plus ou moins renflées nuisent à l'harmonie de la convexité. Cies parties, le plus souvent, ressemblant à des radiations, prennent parfois la forme de zones concentriques.

Les Crlyciana vivent dans les lacs ou les canaux aux eaux limpides bien que peu courantes. Elles sont, à ma connaissance, au nombre de trois (1).

(1) Iruster, sous le nom de ventricosa (pl. XIa fig. 4-5) a donnné la représentation d'une espèce IVurtembergeoise, qui m'est inconnue, que je rapporte néanmoins à ce groupe bien qu'elle soit assez mal rendue. 


\section{Anodonta Giyca.}

Long. $\max . . . \ldots \ldots \ldots \ldots \ldots . . . . .$. 90 mill.

Haut. $\max . . . \ldots \ldots \ldots \ldots \ldots \ldots \ldots$

Epaiss. max. (à 32 des sommets, à 43 du rostre, à 48 du bord antér., à 24 de l'angle postéro-dorsal, à 28 de la base de la perpend..................

Long. de la crête ligamento-dorsale, des sommets à l'angle postéro-dorsal.... 33 -

Dist. de cet angle au rostre......... $421 / 2$

Corde apico-rostrale.............. $731 / 2$

Haut. de la perpendiculaire......... 44 -

Dist. de cette perpend. au bord antér.... $26 \quad$

- du même point de cette perpend. au

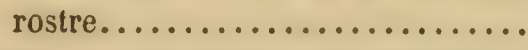

- enfin, de la base de la perpend. à l'angle postéro-dorsal..........

Ghez cette Anodonte, l'épaisseur maximum, plus rapprochée du rostre (43 mill.) que dubord antérieur ( $48 \mathrm{mill}$.), est à 21 mill. en arrière de la perpendiculaire. La hauteur maximum se trouve également en arrière de la porpendiculaire à 30 mill., juste au niveau d'une verticale tombant de l'extrémité du ligament postérieur. Je ferai remarquer, en outre, que l'épaisseur égale, à 2 mill. près, trois fois la longueur maximum et que la région postérieure dépasse de 12 millim., deux fois l'étendue de la région antérieure.

Coq. de forme oblongue, médiocrement aiiongée, ‘̀ région antérieure faiblement dilatée dans le sens de la 
hauteur, irrégulièrement convexe (convexité offrant des míplans on des parties plus renflées les unes que les autres). Valves très brillantes, assez minces, enfin, bâillantes faiblement en avant et d'une façon assez accentuée entre le rostre et l'angle postéro-dorsal.

Bord supérieur rectiligne. Région antéricure arrondie, peu dilatée en hauteur. Bord inférieur descendant presque en ligne droite jusqu'au niveau de la verticale tombant de l'extrémité du ligament postérieur, puis remontant, en s'arrondissant, vers le rostre. Région postérieure dilatée dans le sens de la hauteur, assez allongée et terminée par un rostre très obtus, à peine troncatulé ou plutôt arrondi ; enfin, présendant au-dessus (à partir de l'angle postérodorsal) une direction descendante tantôt rectiligne, tantôt faiblement concave.

Arête dorsale assez régulièrement convexe, indiquée par deux radiations plus foncées des sommets aux extrémités supérieure et inférieure du rostre.

Crête ligamento-dorsale assez saillante, notablement comprimée à l'angle postéro-dorsal qui est carrément coupé et dont l'extrémité forme saillie.

Sommets assez renflés, bien que peu proéminents, sillonnés de rides concentriques tremblotées.

Siries d'accroissement délicates, fines, serrées, légèrement feuilieiées vers les contours.

Epiderme très brillant, d'une teinte jaunacée passant à une nuance cendrée sur la convexité ombonale et à un ton jaune-verdâtre (avec quelques radiations plus vertes) à la région supéro-postérieure. Intérieur d'une nacre bleuâtre irisée.

Ligament antéro-interne, lamelleux, occupant toute l'épaisseur de la région cardinale. Ligament postérieur 
presque entièrement symphynoté, peu allongé, terminé par une longue lunule rectangulaire. Impressions irrégulières, superficielles.

Cetle Anodonte a été recueillie dans le Menthon dans l'Ain (Locard), dans la mare de Tourmont près de Poligny (Jura) ainsi qu'aux environs de Montafroid, également dans le Jura.

Les échantillons de cette dernière localité sont d'une taille plus petite (long max. 78. Haut. $\max 42$. Haut. perpend. 36 , épaiss. max, 28 mill.). Ils soat surtout remarquables par un épiderme d'un jaune plus foncé orné de radiations noires-olivâtres.

\section{Anodonta Doẹi.}

Long $\max . . . \ldots \ldots \ldots \ldots \ldots . . .6105$ mill.

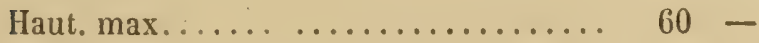

Epaiss. max. (ù $2 \dot{j}$ des sommets, à 59 du rostre, à 49 du bord antér., á 29 de l'angle postéro-dorsal, à 36 de la base de la perpend.).................

Long. de la crête ligam. dors. des sommets à l'angle postéro-dorsal....... 43 -

Dist. de cet angle au rostre.......... 51 -

Corde apico-rostrale............. 800 -

Haut. de la perpendiculaire......... 52 -

Dist. de cette perpend. au bord antér.... 32 -

- du même point de cette perpend. au rostre.................... 76 -

- enifin, de la buse de la perpend. à l'angle postéro-dorsal............ 62 - 
Chez la Doëi, l'épaisseur maximum est plus reporlée vers les sommets que chez l'espèce précédente. Elle est également relativement moins forte et la convexité est plus régulière que chez la glyca.

Coq. de forme oblongue, plus allongée et plus dilatée en hauteur que la précédente, à valves également plus ipaisses, notamment dans toute la région antérieure, et n'offrant qu'un seul båillement assez prononcé entre le rostre et l'angle postéro-dorsal.

Bord supérieur légèrement convexe. Région antérieure médiocre, arrondie. Bord inférieur descendant presque en ligne droite jusqu'à 31 millimètres en arrière de la hase de la perpendiculaire pour remonter ensuite vers le rostre. Région postírieure assez largement allongée, terminée par un rostre carrément troncatulé avec deux angles obtus correspondant aux deux radiations de l'arête dorsale. Entre l'angle postéro-dorsal (qui est saillant et assez aigu) et l'angle supér(o-rostral, le contour suit une ligne descendante un tant soit peu concave dans sa partie supérieure.

Arête dorsale peu accentuée, régulièrement convexe, descendant d'une façon normale ver's la créte ligamentodorsale, et, pourvue de deux radiations verdâtres divergentes du sommet au rostre, où elles aboutissent aux deux angles obtus du rostre.

Crête ligamento-dorsale assez saillante, comprimée, surtout vers la région de l'angle postéro-dorsal qui est carrément coupé.

Sommets non renflés, à peine proéminents, comme écrasés, à crochets très aigus, sillonnés par de fines rides saillantes fortement tremblotées.

Stries d'accroissement délicates, serrées, légèrement feuilletées seulement vers la région des contours. 
Épiderme très brillant, d'un jaune clair avec quelques zones concentriques plus foncées, oṛné, en outre, de très fines radiations vertes, devenant, vers la région postérieure, si pressées, qu'elles donnent à cette portion des valves une teinte verte uniforme. Vers la région du sommet la coloration, d'un ton cendré pàle tirant sur une nuance rosacée, passe vers les crochets à une teinte jaune paille.

Intérieur d'une nacre très irisée blanchâtre offrant des miroitements tantôt bleuâtres, tantòt verdâtres avec des 'írisations de l'arc-en-ciel.

Ligament antéro-interne, lamelleux, occupant toute l'épaisseur de la région cardinale. Ligament postérieur non saillant, presque toujours entièrement recouvert, terminé par une longue lunule de 14 millimètres de forme subtétragone. Impressions accentuées, surtout l'antérieure et la palléale.

Cette espèce que je dédie à notre ami Ferdinand Doë, vit dans lés cunaux du parc du château des Cours, à SaintJulien, à 4 kilom. en amont de Troyes (Aube).

Je me rappelle avoir vu autrefois, dans la collection de M. Drouët, quelques échantillons de cette Anodonte étiquetés sous le nom de subponderosa de Dupuy.

\section{Anodonta lacuum.}

Anodonta lacuum, Bourguignat (royez page 103), mai 1880.

('est cette espèce qui a été figurée par Brot (Näades du Léman), d'abord pl. VI, fig. 1 et 4 , sous les noms d'anatina elongata et de cellensis var. dilatata, puis pl. VIII, fig. 3 , 
sous l'appellation de Pictetiana var. elongata. Ces trois figures, parfaitement bonnes et très exactes, représentent très bien celte espèce et suffisent à la connaisssance de celte Anodonte.

La Lacuum a été recueillie en Suisse eten Savoie : $1^{\circ}$ Dans les marais du Rhône, à partir de Villeneuvejusqu'à SaintMaurice dans le Valais (Brot. loc. sup. cit.p. 37); $2^{\circ}$ dans le lac Genève et dans les fossés de la ville de Genève du côté de la Coulouvrenière (Brot, p.44); $3^{\circ}$ dans le lac de SaintPaul, au-dessus de Thonon (Brot, p. 46); $4^{\circ}$ enfin, dans le Rossenback près d’Ebikon, d'où M. Brot l'a adressée autrefois, sous le nom de cellensis var. ovala, à notre ami le professeur Deshayes, qui nous a communiqqué cette espèce.

La Lacuum n'est jamais lisse et brillante comme les Glyca et Doēi. Ses valves sont toujours salies par un enduit terreux noirâtre. Lorsque cet enduit est enlevé, l'épiderme offre une belle teinte verte, surtout sur la région postérieure.

\section{go GALLANDIANA.}

Les espèces de ce groupe sont des formes allongées, généralement peu hautes, d'un teinte terne, toujours d'une nuance foncée brune ou olivâtre, tantôt ventrues (Mahometana, Tchernaica), ou tantôt peu convexes (ectina, crimeana), à ligament postérieur presque entièrement recouvert. Les sommets sont très renflés chez les Gallandi, Ma hometana et Tchernaica et à peine proéminents chez les ectina et Crimeana.

Cies Anodontes sont, en outre, caractérisées par des 
valves offrant un bâillement très considérable sur toute l'étendue du bord palléal. Ce bâillement est parfois si accentué que les valves sont entr'ouvertes depuis les sommets jusqu'au rostre, et, comme il existe un autre bâillement au-dessus du rostre, il résulte de là que les valves ne se touchent que dans l'étendue des ligaments et à l'extrémité postéro-rostrale.

Les Gallandiana vivent dans les eaux de la Crimée et paraissent, de plus, assez répandues dans les ruisseaux du Bosphore.

\section{Anodonta Gallandi.}

J'ai donné, page 95 , les signes distinctifs de cette Anodonte. Elle habite indifféremment en Asie, les eaux de la côte anatolique, et en Europe, celles de la rivière d'Alibey, qui se jettent à l'extrémité du port de Constantinople.

\section{Anodonta mahometana.}

Long. $\max . . . . \ldots \ldots \ldots \ldots \ldots \ldots . .64$ mill.

Haut. $\max . \ldots \ldots \ldots \ldots \ldots \ldots \ldots . \ldots \ldots, 451 / 2$

Épais. max. (à 21 des sommets, à 45 durostre, à 40 du bord antér., à 18 de l'angle postéro-dorsal, à 29 de la base de la perpend.).........................

Long. de la crête ligam.-dors. des sommets à l'angle postéro-dorsal:............ 30 1/2

Distance de cet angle au rostre......... 41 -

Corde apico-rostrale............... 67 -

Haut. de la perpendiculaire........... 40 - 
Dist. de cette perpend. au bord antérieur. 25 mill.

— du même point de cette perpend. au rostre..................... 61 -

- enfiu, de la base de la perpend. à l'angle postéro dorsal................. 45 -

Chez cette espèce de taille fort médiocre, taille bien inférieure à celle de la Gallandi, la convexité maximum, rapprochée du bord supérieur, décroît d'une façon régu. lièrement convexe aussi bien en avant que vers les bords inférieur et postérieur. Elle n'offre point, comme chez la Gallandi, cette décroissance plane, presque tectiforme, qui donne à cette espèce un cachet tout particulier.

Coq. allongée-oblongue, à conlours sans angles saillants, relativement très bombée pour sa taille, à valyes peu épaisses et fortement bâillantes postéricurement en dessous de l'angle postéro-dorsal et en avant depuis la partie supéro-antérieure jusqu'au rostre.

Bord supérieur assez court, presque rectiligne. Région antérieure arrondie. Bord inférieur clescendant presque en ligne droite (sauf une légère sinuosité) jusqu’à உŏ mill. en arrière de la perpendiculaire, pour de là remonter par un contour arrondi vers le rostre. Région postérieure très allongée, plus de deux foịs plus longue que l'antérieure, termince par un rostre obtus (sans partie anguleuse) regardant un peu en bas, et, offrant en dessus jusqu'à l'angle postéro-dorsal un contour légèrement concave.

Sommets gros, ventrus, proéminents, sillonnés, lorsqu'ils ne sont pas excoriés, ce qui arrive souvent, par de fines costulations tremblotées.

Arête dorsale accentuée surtout vers les sommets, devenant ensuite indécise vers la région postérieure. Grête dorsale peu prononcée, non comprimée. 
Stries d'accroissement très délicates sur le milieu de la convexité et très feuilletées sur tous les contours, notamment en avant et en arrière.

Épiderme toujours encrassé d'un limon ferrugineux, sauf sur la région ventrale où il apparaît d'une teinte jaune-olivâtre, passant vers les sommets à un ton rougebrique. Intérieur d'une nacre si éclatante qu'elle reflète toutes les couleurs de l'arc-en-ciel.

Ligament antérieur, interne, lamelleux, occupant toute l'épaisseur de la charnière et se poursuivant, au-dessous de l'autre ligament, très loin en arrière du crochet. Ligament postérieur à peine saillant, presque entièrement symphynoté, présentant à son extrémité une assez grande lunule de forme triangulaire.

Cette espèce a été recueillie à Constantinople par l'ingénieur Jules Galland, dans un petit ruisseau, près de la pointe du sérail.

\section{Anodonta Tehernaica.}

Long. $\max . . . \ldots \ldots \ldots \ldots \ldots \ldots, 92$ mill.

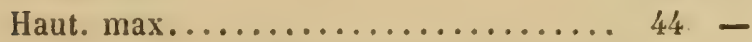

Épaiss. max. (à 22 des sommets, à 44 du rostre, à 41 du bord antér., à 17 de l'angle postéro-dorsal, à 27 de la base de la perpend.).............. 31 -

Long. de la crête ligamento-dorsale des sommets à l'angle postéro-dorsal.... 36 -

Dist. de cet angle au rostre........... 37 -

Corde apico-rostrale............. 69 -

Haut. de la perpendiculaire......... 43 - 
Dist. de cette perpend. au bord antér... 30 mill.

- du même point de cette perpend. au rostre...................... 63 -

- enfin, de la base de la perpend. à l'angle postéro-dorsal............... 52 -

Celte espèce est remarquable par sa forme allongée à bords supérieur et inférieur d'une égale convexité, et surtout par sa ventrosité se faisant sentir dans le sens de la longueur et dont le maximum, plus rapproché des sommets et de l'angle postéro-dorsal que du contour inférieur, se trouve presque à la même distance du rostre et du bord antérieur. Ghez cette Anodonte, les sommets sont plus médians que chez les deux espèces précédentes, et la région postérieure n'est guère plus haute que l'antérieure.

Coq. oblongue-allongée, à contours sans angles saillants, relativement bien ventrue (convexité normale dans le sens de la longueur), à valves assez épaisses, présentant les deux bâillements caractéristiques du groupe.

Bord supérieur assez long, un tant soit peu convexe. Région antérieure arrondie, bien développée. Bord inférieur légèrement convexe, à son extrémité remontant vers le rostre. Région postérieure allongée, ne se dilatant pas en hauteur, conservant à peu près la même proportion qu'au niveau de la perpendiculaire, se terminant par un rostre obtus, arrondi, sans angles saillants et offrant audessus du rostre, à partir de l'angle postéro-dorsal, un contour descendant presque en ligne droite.

Sommets gros, ventrus, proéminents, très obtus; enfin, très finement ridés.

Arête dorsale ventrue, surtout vers les sommets, s'atté- 
nuant régulièrement vers la région postérieure. Crête dorsale presque nulle, non saillante.

Stries d'accroissement délicates, sauf vers les contours où elles deviennent plus ou moins feuilletées.

Epiderme brillant, d'une couleur foncée jaune-noire olivâtre, avec quelques fines radiations peu perceptibles. Intérieur d'une nacre lactescente bleuacée assez bien irisée.

Ligament antérieur, interne, foliacé, se poursuivant en arrière des crochets, presque jusqu'à moitié du ligament postérieur, qui est assez mince, non saillant, entièrement symphynoté et terminé par une toute petite lunule ressemblant à une virgule.

Celte Anodonte vit dans les eaux de la Tchernaia, près de Sébastopol, en Grimée.

\section{Amodonta Crimeana.}

Cette nouvelle forme, qui a été également recueillie dans la Tchernaia, se distingue de la précédente par ses valves très comprimées, plus minces, moins pesantes, possédant un contour régulièrement ovoïde-allongé; par ses sommets bien plus petits, à peine saillants, comme écrasés; par son arête dorsale presque nulle; par ses bords supérieur et inférieur plus arqués; par sa crête dorsale pas plus développée que celle de la Tchernaica, mais en revanche, assez comprimée, etc.; enfin, par sa taille moindre.

Long. $\max . . . \ldots \ldots \ldots \ldots \ldots \ldots, 73$ mill.

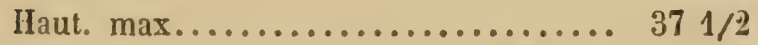

Épaiss. max. (à 14 des sommets, à 41 du 3AI. 1881. 
rostre, à 34 du bord antér., à 16 de l'angle postéro-dorsal, à 29 de la base de la perpendiculaire)........... 19 mill.

Long. de la crête ligam.-dors. des sommets à l'angle postéro-dorsal....... 26 -

Dist. de cet angle au rostre.......... 32 -

Corde apico-rostrale............... 56 -

Haut. de la perpendiculaire.......... 35 -

Dist. de cette perpend. au bord antér..... 23 1/2

- du même point de cette perp. au rostre. 50 -

- enfin, de la base de la perpend. à

l'angle postéro dorsal.......... 41 -

On remarquera que la Crimeana n'a que 19 d'épaisseur, lorsque la Tchernaica a 31 mill.; que la convexilé de cette espèce est plus rapprochée des sommets et du bord antérieur que celle de l'Anodonte précédente.

\section{Anodonta ectina.}

Cette Anodonte, de la taille de la Crimeana, aussi aplatie qu'elle et à peu près de même coloration, s'en distingue cependant d'une façon tranchée, par son bord supérieur rectiligne, non arqué; par son bord inférieur convexedeșcendant ; par sa région postérieure plus dilatée dans le sens de la hauteur, puisque sa hauteur maximum (41 mill.) se trouve à 20 mill. en arrière de la perpendiculaire qui n'a que 38; par son rostre plus comprimé, moins obtus, dont l'extrémité regarde un peu en bas, tandis que celui de la Crimeana se dirige dans le sens horizontal; par sa crête dorsale plus comprimée; par son angle postérodorsal plus saillant, carrément coupé sous un angle assez 
accentué; par sa convexité maximum plus centrale, s'atténuant de tous côtés d'une façon rérulière et plus prononccee. Chez la Crimeana, la convexité, moins centrale, s'atténue moins vite et se conserve sur toute la partie médiane des valves, de telle sorte que les contours, moins comprimés, sont plus aigus.

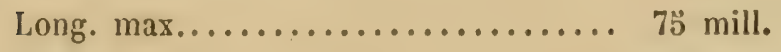

Haut. $\max . . \ldots \ldots \ldots \ldots \ldots \ldots . . . .41$ -

Épaiss. max. (à 19 des sommets, à 39 du rostre, à $3 د$ du bord antér., à 20 de l'angle post.-dors., à 24 de la base de la perpendiculaire $) . . . \ldots \ldots \ldots \ldots . .19$ -

Long. de la crête ligam. dors. des sommets

à l'angle pustéro-dorsal........... 26 -

Di it. de cet angle au rostre.......... 37 -

Gorde apico-rostrale............. 58 -

Haut. de la perpendiculaire.......... 38 -

Dist. de cette perpend. au bord antérieur. $23 \quad 1 / 2$

— du même point de cette perpend. au rostre................... 52 -

- enfin, de la base de la perpend. à l'angle postéro-dorsal............ 43 -

L'Ectina a été découverte par l'ingénieur Jules Galland dans un petit cours d'eau, vers la pointe du sérail, à Constantinople.

\section{BRACHLTEVTUANA}

Les Anodontes de ce groupe, bien que difficiles à caractériser, sont cependant faciles à reconnaître à leur air de 
parenté. Elles paraissent spéciales à l'Espagne et au Portugal, ainsi qu’à quelques localités du sud de la France et du nord de l'Afrique. La Lucasi (Deshayes, 1847) et l'embia (Bourguignat, 1864), de la province de Constantine, appartiennent, en effet, à ce groupe 1 .

Les Macilentiana ont des valves épaisses, parfois très pesantes (suivant les espèces), et, non ou à peine bâillantes. Chez la plupart, les bâillements sont nuls. Toutes (sauf les Castroi et Castropsis) offrent une sinuosité au bord inférieur, sinuosité quelquefois très prononcée, comme chez les macilenta et submacilenta.

\section{Anodonta macilenta.}

Anodonta macilenta, Mforelet, Moll. Portug., p., 102, pl. XI, f. 1, 1845 .

Belle espèce portugaise parfaitement représentée dans l'ouvrage de Morelet.

Elle est abondante à 4 kilom. de Coimbre dans les marécages de la Valla de Géria, près du Montégo.

\section{Anodonta submacilenta.}

Anodonta submacilenta, Servain, Moll. Esp. et Portug., p. 162. 1880.

Cette Anodonte, dont le $\mathrm{D}^{\mathrm{r}} \mathrm{G}$. Servain a bien fait ressortir tous les caractères, habite dans le grand étang

1. Les Tunizana, numidica et Letourneuxi d'Algérie n'en font pas partio. 
d'Albuféra, près de Valence, ainsi que dans les étangs pyrénéens des vallons au-dessus de Port-Vendres, dans les Pyrénées-Orientales, où elle a été recueillie par notre ami le $D^{r}$ Penchinat.

\section{Anodonta Penchinati.}

Long. $\max . . . \ldots \ldots \ldots \ldots \ldots \ldots . . . .125$ mill.

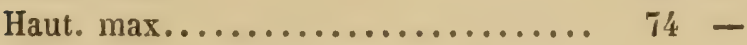

Épais. max. (à 38 des sommets, à $6 \mathbf{5}$ du rostre, à 61 du bord antér., à 36 de l'angle postéro-dorsal, et, à 36 également de la base de la perpend.)...... Long. de la crête ligamento-dorsale des sommets à l'angle postéro-dorsal...

Distance de cet angle au rostre....... 67 -

Corde apico-rostrale............. 100 -

Hauteur de la perpendiculaire....... 67 -

Dist. de la perpend. au bord antér..... $45^{\circ}-$

- du même point de la perpend. au rostre.................... 83 -

- enfin de la base de la perpend. à l'angle postéro-dorsal............ 70 -

Chez cette Anodonte, l'épaisseur (50 mill.), ainsi que la hauteur maximum ( $i 4$ mill.), comparées à la plus grande longueur (19马 mill.) sont relativement énormes. On remarquera de plus que le maximum de la convexilé est presque central, puisqu'il est, à 3 mill. près, aussi distanl du rostre qus du bord antérieur, et à 2 mill. près, aussi éloigné des sommets que de la base de la perpen- 
diculaire. Chez la submacilenta, la convexité est plus rapprochée de l'extrémité rostrale que du bord antérieur.

La Penchinati est une espèce très ventrue, peu dilatée antérieurement, légèrement sinueuse infírieurement au niveau de la perpendiculaire, à région postérieure relativement écourtée dans le sens de la longueur, dilatée au contraire, dans celui de la hauteur, et, offrant, en somme, une forme irrégulièrement ovalaire dans une direction obliquement descendante. Sa convexité, dont le point maximum est presque central, ainsi que je l'ai dit, est surtout accentuée sur la zone de l'arête dorsale. Des sommets au rostre, la convexité offre, en effet, sur cette zone, une ventrosité très accentuée, ventrosité qui diminue d'une façon assez régulière vers la partie antérieure et palléale, mais qui descend presque à pic sur la région de la crête dorsale.

Comme contour, la Penchinati ressemble assez à la $m a$. cilenta du Portugal, dont elle diffère notamment par sa grande convexité (la macilenta, d'après Norelet, est aplatie); par ses sommets plus gros, plus ventrus et très proé. minents ; par sa sinuosité inférieure juste au niveau de la perpendiculaire, tandis que celle de la macilenta se trouve plus portée en arrière de cette ligne. J'ajouterai encore qu'à l'endroit où chez la macilenta, le bord inférieur éprouve la sinuosité si bien rendue dans la gravure de Morelet, se montre, au contraire, chez la Penchinali, une dilatation très accentuée. Chez la macilenta, enfin, la région postírieure a une direction obliquement descendante bien plus prononcée ${ }^{1}$ que chez notre espèce.

1. Je rappellerai que toutes les Anodontes que je décris sont placées de façon à ce que les sommets soient culminants (roir ma méthode de mensuration). Ainsi placée, la macilenta a un tout autre 
La Penchinati est encore très différente de la submaci. lenta de notre ami le $\mathrm{D}^{\mathrm{r}}$ Servain.

La submacilenta est une espèce également très ventrue, mais dont la convexité maximum n'est pas centrale. Sa forme est allongée dans un sens non descendant, mais horizontal, avec une très forte sinuosité en arrière de la perpendiculaire à sa partie postéro-palléale. Chez cette Anodonte, la région antérieure est très développée comme hauteur, tandis que sa région postérieure est contractée. C'est justement le contraire chez la Penchinati et la macilenta.

Valves épaisses, assez pesantes, à peine bâillantes en avant et en arrière au dessus du rostre (les bâillements souvent ne sont pas perceptibles).

Bord supérieur légèrement convexe. Région antérieure médiocre, arrondie. Bord inférieur presque rectiligne, descendant, avec une légère sinuosité à l'endroit de la perpendiculaire et présentant, à son extrémité, une dilatation, pour de là remonter vers le rostre. Région postérieure dilatée dans le sens de la hauteur, peu allongée, terminée par un rostre excessivement obtus, arrondi, sans angles saillants, offrant en dessus, depuis l'angle postéro-dorsal, une direction rectiligne descendante.

Arête dorsale très proúminente, très convexe, descendant presque à pic sur la crête dorsale qui est nulle.

aspect que celui que Iui a donné Morelet, sur sa planche. Son bord supérieur, en effet, au lieu d'être ascendant, devient faiblement convexe sur un plan presque horizontal; le bord inférieur, de rectiligne un peu sinué, accuse, au contraire, une direction descendante très prononcée. En somme, cette Anodonte offre une forme suboblongue dans un sens obliquement descendant de gauche à droite, à compter des sommets. 
Sommets très convexes, gros, obtus, proéminents (tou. jours excoriés).

Stries presque lisses sur la convexité (avec des zones concentriques d'accroissement çà et là plus fortes), devenant, vers les contours, plus accentuées, souvent rugueuses et feuilletées. Ĺpilerme brillant, d'un brun rougeâtre passant au rouge-brique vers les sommets, au noir vers la région postérieure et quelquefois au jaune vers le bord palléal. Intérieur d'une belle nacre bleuâtre bien irisée.

Ligament antéro-interne exigu et délicat. Ligament postérieur court, gros et trapu, presque entièrement recouvert, terminé par une lunule marron, bien triangulaire.

Région cardinale de la charnière légèrement sinuée, très mince. Région latérale plus forte et plus épaisse.

Impressions très superficielles (sauf la palléale) et souvent difficile à saisir.

Cette belle Anodonte a été recueillie, en 1863, par notre ami le $\mathrm{D}^{\mathrm{r}}$ Penchinat, auquel je la dédie, dans les étangs des vallons pyrénéens, au-dessus de Port-Vendres, dans les Pyrínées-Orientales.

\section{Anodonta Martorelli.}

Anodonta Martorelli, Bouryuignat, in : Servain, Moll. Lsp. et Portug., p. 166, 1880.

Cette espèce, à laquelle j'ai attribué le nom de notre regretté ami, récemment décédé, Francisco Martorell y Peña, de Barcelone, vit dans les eaux du grand étang d'Albuféra, près Valence.

La Martorelli, épaisse et pesante, est une forme à bords 
supérieur et inférieur presque parallèles (avec une légère sinuosité au bord palléal); à région antérieure dilatée dans le sens de la hauteur et à région postérieure presque aussi large et aussi développée que l'antérieur'e. Chez cette espèce, la hauteur maximum (68 mill.) coïncide juste avec la ligne perpendiculaire. Les valves sont régulièrement convexes, arec une ventrosité (44 mill.) normale pour sa grandeur (long. 124 mill.). La région rostrale, très obtuse, a une légère tendance à être un tant soit peu remontante, tandis que celle des Anodontes précédentes, sauf la submacilenta, offrent une direction un peu descendante.

\section{Aodonta viriata.}

Anodonta viriata, Servain, Mull. Esp. et Portug., page 169,1880 .

La Viriata est une Anodonte ovalaire, à valves épaisses et pesantes, bien régulièrement ventrue et dont la convexité maximum (42 mill.), à égale distance de l'angle postéro-dorsal et de la base de la perpendiculaire, se trouve plus rapprochée du bord antérieur que chez toutes les espèces précédentes.

Les sommets, obtus, arrondis, non proéminents, sont sillonnés de fortes rugosités espacées. La région latérale de la charnière est très exiguë, plane, très robuste, avec un sillon à son extrémité.

Cette espèce habite également dans le grand étang d'Al. buféra, près de Valence (Servain). 


\section{Anodonta melíia.}

Anodonta melinia, Bourguignat, Noll. nouv. (5 décade 1865), $n^{\circ} 500$, p. 154 , pl. XXVIII, f. 1-5, - et, in : Servain, Moll. Esp. et Portug., p. 160, 1880.

Chez la melinia, le ligament postérieur est totalement symphynoté; l'épiderme est d'une teinte violacée assez remarquable, et, entre l'angle postéro-dorsal et le rostre, s'ouvre un bâillement relativement considérable.

La représentation que j'ai donnée, pl. XXVIII, de cette Anodonte suffit grandement à la connaissance de cet Acéphale.

Dans le temps (1815), lorsque j'ai publié celte espèce, alors que j'étais sous l'empire des idées erronées qui avaient cours à cette ćpoque et que j'étais moins ferré que je ne le suis sur les caractères des acéphalés, j'avais désigné celte bivalve comme appartenant au groupe de la piscinalis, actuellement je reconnais que la melinia ne peut faire partie de ce groupe.

Cette Anodonte est abondante dans le grand étang d'Albuféra, si riche en espèces de tous genres, ainsi que dans divers étangs de la magnifique Huerta de Valence.

\section{Anodonta Castroi.}

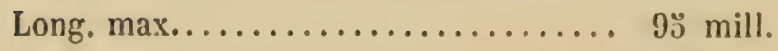

Haut $\max . . . \ldots \ldots \ldots \ldots \ldots$.......

Épaiss. max. (à 27 des sommets, à 47 du rostre, à 49 du bord ant., à 26 de l'angle 
postéro-dorsal, à 37 de la base de la perpendiculaire) ............... 30 mill.

Long. de la crête ligamento-dorsale, des sommets à l'angle postéro-dorsal..... 33 -

Dist. de cet ang!e au rostre.......... 42 -

Corde apico-rostrale.............. 72 -

Haut. de la perpendiculaire......... 52 -

Dist. de cette perpend. au bord antérieur. 32 ..

- du même point de cette perpend. au rostre................... 62 -

- enfin, de la base de la perpend. à l'angle postéro-dorsal.........6 61 -

Chez la Castroi, l'épaisseur maximum est plus rapprochée du rostre que du bord antérieur et sa région postérieure n'est pas tout à fait deux fois aussi longue que l'antérieure.

Cette Anodonte, d'une forme irrégulièrement subtétragone, peu ventrue, très développée en hauteur relativement à sa longucur, possède des valves bien brillantes, assez épaisses, et peu bâillantes en avant et en arrière.

Burd supérieur subrectiligne, un tant soit peu arqué. Région antérieure arrondie, bien développée en hauteur. Bord inférieur médiocrement convexe, dans une direction légèrement descendante. Région postérieure haute, peu allongée, terminée par un rostre assez aigu, bien qu'obtus, situé vtrs la partie inférieure, et, présentant entre ce rostre et l'angle postéro-dorsal un contour rectiligne obliquement descendant.

Arête dorsale normalement convexe, offrant deux rayons divergeant du sommet au rostre. Crête dorsale courte, assez notablement comprimée vers le lig:ment et vers l'angle postéro-dorsal. 
Sommets écrasés, à peine proéminents (à crochets dont la pointe est aiguë et saillante), et sillonnés par des rides concentriques très espacées et légèrement tremblotées.

Stries presque lisses ou très fines, devenant de plus en plus fortes vers la région des contours et même feuilletíes à la partie postérieure. Épiderme brillant, quelquefois d'un jaune d'ocre uniforme plus ou moins foncé, d'autre fois d'un cendré grisâtre sur la région ombonale, passant au jaune vers les contours antérieurs et au vert (avec quelques radiations plus vertes) sur la partie postérieure. Intérieur d'une belle nacre irisée d'un ton bleuâtre ou d'un pâle rosacé.

Ligament antéro-interne lamelleux, très mince. Ligament postérieur médiocre, toujours symphynoté, terminé par une lunule médiocre, subtriangulaire.

Région latérale de la charnière forte et blanchâtre, tandis que la cardinale est nulle. Impressions : antérieure subtriangulaire très allongée; postérieure en forme de palette.

Cette espèce, dédiée au malacologiste portugais José da Silva e Castro, a été recueillie aux environs de Valence, dans les étangs, notamment dans celui d'Albuféra.

\section{Anodonta castropsis.}

Anodonta castropsis, Fagot, mss. 1880.

Cette nouvelle Anodonte provient des bassins du Capitany, près de Montferrand, dans le département du Gers, d'où elle a été envoyée par l'abbé Dupuy, sous le nom crroné de subponderosa. Je suis à me demander comment 
l'honorable abbé a pu assimiler cette forme, si particulière, à la subponderosa publicée dans son histoire des Mollusques de France.

La Castropsis de notre ami Fagot est une espèce de type hispanique, voisine de la Castroi, ainsi que l'indique son nom. Un peu plus grande et d'une forme un peu plus subtétragone que l'Anodonte espagnole, la Castropsis possède des valves plus épaisses. Son bord supérieur est rectiligne; l'inférieur est moins convexe; la partie rostrale plus largement obtuse; le contour, entre le rostre et l'angle postéro-dorsal, un tant soit peu concave et non rectiligne. Les sommets, enfin, tout en étant plus gros et plus obtus, sans être plus proéminents, ont des crochets moins saillants et offrent des rides moins nombreuses, plus serrées et moins fortes.

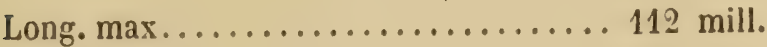

Haut. max.................... 63 -

Épaiss. max. (à 30 des sommets, à 56 du rostre et du bord ant., à 29 de l'angle postéro-dorsal, à 39 de la base de la perpendiculaire $). . . \ldots \ldots \ldots \ldots \ldots \ldots$

Long. de la crête ligamento-dorsale, des sommets à l'angle postéro-dorsal.... 38 -

Dist. de cet angle au rostre.......... 52 -

Corde apico-rostrale.............. 85 -

Haut de la perpendiculaire.......... 61 -

Dist. de cette perpend, au bord antér.... 40 -

- du même point de cette perp. au rostre..................... 74 -

- enfin, de la base de cette perpend. à l'angle postéro-dorsal.......... 68 - 
On remarquera que chez cetle espèce, relativement peu ventrue (convexité régulière), ainsi que la précédente, l'épaisseur maximum se trouve juste à égale distance des extrémités postérieure et antérieure, tandis que chez la Castroi, le maximum est plus rapproché du rostre que du bord antérieur. On remarquera encore que si la distance des sommets à l'angle postéro-dorsal, est de 38 mill. chez les deux espèces, la distance de cet angle au rostre est, chez la C'astropsis de Fagot, de 52, Jorsqu'il n'est que de 42 chez la Castroi. Il résulte des chiffres de celte mesuration que la région de la crête ligamento-dorsale est relativement, chez la Castropsis, moins allongée que chez la Castroi. J'ajouterai que la hauteur maximum (63 mill.) se trouve à 38 mill. en arrière de la perpendiculaire, juste au niveau d'une verticale tombant de l'angle postérodorsal, tandis que celle de la Castroi (วัö mill.) n'est que de 22 mill. en arrière de la perpendiculaire.

\section{Do ADAMIANA}

Intermédiaires entre les formes hispaniques des Macilentiana et celles des Ponderosiana du centre de l'Europe, les Adamiana semblent spéciales au nord et au centre de l'Italie, ainsi qu'aux lacs du sud du Tyrol.

Les espèces de ce groupe doivent être fort répandues et vraisemblablement fort nombreuses dans l'Italie septentrionale. Je n'en connais que trois malheureusement. Ces Anodontes sont très brillantes, bien ventrues, à convexité régulière; les valves sont relativement assez épaisses et n'offrent qu'un bâillement postérieur assez médiocre; l'épiderme est toujours d'une nuance très foncée, variant 


\section{$-191-$}

d'un cendré-violacé ou olivâtre à un cendré-noirâtre, s'éclaircissant sur la région ombonale où il prend un ton jaunacé-rougeâtre.

De forme oblongue, plus ou moins ovoïde, les Adamiana, bien que ne possédant aucun signe particulier nettement saillant, qui permette de les caractériser d'une façon tranchée, ne forment pas moins un groupe à part, essentiellement italien, que je n'ai pu confondre avec aucun autre du système européen.

\section{Anodonta Adamil.}

Long. $\max \ldots \ldots \ldots \ldots \ldots \ldots \ldots, 104$ mill.

Haut. $\max . . . \ldots \ldots \ldots \ldots \ldots \ldots . .58$ -

Épaiss. max. (à 30 des sommets, à 504 du rostre, à 52 du bord antér., à 27 de l'angle postéro-dorsal, à 37 de la base de la perpendiculaire)............ 34 -

Long. de la crête ligam.-dors., des sommets à l'angle postéro-dorsal....... 40 -

Dist. de cet angle au rostre........... 50 -

Haut. de la perpendiculaire.......... 56 -

Dist. de cette perpend. au bord antér.... 33 -

- du même point de cette perpend. au rostre..................... 71 -

- enfin, de la base de la perpend. à l'angle postéro-dorsal........... 62 -

Espèce de forme oblongue, avec une partie postérieure relativement assez allongée dans une direction faiblement descendante, à valves assez minces, bâillantes seulement 


\section{$-192-$}

antre le rostre ef l'angle postero-torsal, et remarquables par des stries diacroissement en forme de cobles très rigulietres, émouseces, bien distantes les unes des autres et sillonnant toute la région ventrale de la fagon la plus élésante.

Bord supérieur lécirement arqué. Rigion antérieure arrondie, bien déreloppée. Bord inférieur faiblement convexe-descendant, puis remontant rers son extrimité par une courbe accentuce vers le rostre. Rigion postérieure assez allongede. plus de deur fois plus longue que l'antirieure. termines par un rostre arrondi. très obus, relatirement assoz comprimé et offrant en dessus de ce rostre à partir de lansle postéro-dorsal une direction concavedescendante.

Sommets trds obtus, a peine proiminents, comme cerasés, sillounes de rides trembloties et otirant des crochets tris rigus ot sensiblement saillants. Arête dorsale, sans caracteres particuliers, confondue dans la convexité (rígulière) des valves. Crète dorsale peu développée, assez amincie vers l'angle postérontorsal.

Stries en forme de côtes rigulieres sur foute la région ventrale, devenant, vers les contours, cordées, assez srossieres et faiblement fouilletes.

Epiderme bien brillant, d'un cendré noiràtre sur la partie moyenne, passant sur la rérion ombonale à une nuance rouge-jaunacce et rers les contours à celle d'un ton tantôt jaunåtre tantôt verdâtre; entin, odirant sur toute sa surface des multitudes de petites radiations filiformes d'une teinte plus foncée. Intérieur d'une belle nacre bleuatre.

Ligament antero-interne, puissant, occupant toute l"é. paisseur de la region cardinale, sur laquelle on remarque un très faible rentlement tuberculeux. Ligament postérieur 
fort saillant, prestue entisement symphyonofe pe formint par uno lunule triangulairo.

Impressions : antérioure profonde, irréguliorement sub. tétragono; postéricure superficiolle, en formo do paletto.

Getto Anodonte, que je me litis un plaisir do dídior au capitaine Giov. Batt. Adami, auteur d'excellents travaux malacologiques sur la fauno italionno, a élé recueillie, en 'Toscane, daus la Bientina.

\section{Anodonts do Eottanss.}

Anodonta do Bettana, Martinati, mss.

Anodonta leprosa 1 var. de Bettana, Gredler, 'T'irol's conch. 11 abth. 1859, p. 48, f. 2, B. C. (nom de Bettana de Clessin et do tous les auteurs).

Cetto Anodonto est surtout caractérisée pax son arête dorsale pourvue de deux sillons tris saillants, divergeant des sommets au rostro. Ces sillons, ressemblantà de fortes côtes, descendent en ligne droite sur la partie ro:trale, où

1. Jo ne connais pas l'Anod. loprosa do l'arreyss, signalúe (sans caractères) dans les cours d'oaux du P'iémont par Villa (I)isp. syst. conch., p. 40. 184.) - Gredlex ('Tirol's conch.) rango, sous co nom, l'idrina do Spinelli et mémo les Benacensis et de Bellana, co qui est errone. - Clessin (Anod. in : 2mo édit. de Chemnitz, p. 168, 1876) place la leprosa en synonymio de l'idrina, dans laquelle il aduet quatre variétes : 10 la Benacensis do Villa; ¿o la leprosa de Parreyss; 3o la ventricosa do Spinelli (non C. Pleifler), qui m'est inconnue; et 40 la De Beltana do Martinati. - La leprosa, d'apres Clessin, est une petite espece ovoïde, de teinte brune, a sommets excories. Jo crois que cette petito Anodonto est uno formo distincto et spéciale au Piémont. Jo penso qu'ello doit appartenir au groupo do l'Abbrcviatiana et qu'elle doit êtro voisine des fallax et psammita. 
ils donnent lieu à deux angles prononcés qui font paraitre le rostre comme troncatulé. D'après la figure, fort bien faite, donnée par Gredler, la région postérieure, près de trois fois plus longue que l'antérieure, parait fortement descendante.

Cette espèce vit dans le petil lac de Montikler (Tyrol). Gredler la cite encore des lacs de Kalterer et de Loppio ; mais je crois que les Anodontes de ces dernières localités sont dissemblables de la de Bettana du lac Montikler.

Clessin (Anod. in : $2^{\text {mo }}$ édit. Chemnitz, p. 167) et l'auteur des suites à Rossmässler (Iconog., pl. 120, fig. 1156 à 1159) ont décrit et fait figurer, comme variété de l'idrina, des de Bettana qui ne peuvent être rapportées à la vraie de Beltana représentée par Gredler.

\section{Anodonta Loppionica.}

J'ai reçu cette espèce du lac Loppio dans le sud du Tyrol, sous le nom de de Bettana. Cette Anodonte est differente de celle de Martinati, figurée par Gredler.

Notre loppionica ressemble, au contraire, à l'acéphale représenté dans les suites à Rossmässler (fig. 1159) sous l'appellation erronée d'idrina et décrit, dans le même ouvrage (p. 69. no 1159), sous le nom de de Bettana. Cette espèce, comme la nôtre, provient du lac Loppio. Sauf l'arête dorsale qui semble dans la figure $115 \% 9$ un peu plus accentuée que celle de mes échantillons, et, sauf une taille un peu moindre, je ne vois pas d'autres différences entre cette figure et mes échantillons.

- L'auteur de ces suites à Rossmässler a réuni sous le nom d'idrina : $1^{\circ}$ fig. 1157 , l'idrina de Spinelli, espèce italienne 
qui sert de type de groupe; $2^{\circ}$, fig. 1156 , la sebinensis d'Adami, espèce du groupe des rostrata; $3^{\circ}$ fig. 1158 , une forme bien voisine de la Benacensis de Villa, espèce du groupe des Arealiana; $4^{\circ}$ enfin, fig. 1159 , notre loppionica, qui appartient à celui des Adamiana.

\section{H (Do PoNDERosiana}

Les Ponderosiana sont des Anodontes à valves généralement épaisses, quelquefois très pesantes, recouvertes d'un épiderme sombre, très foncé, variant d'un cendré. brunâtre ou olivâtre à un noir-verdâtre ou rougeâtre. Les sommets sont très volumineux et proéminents chez la Mabilli, un peu moins chez la vraie ponderosa; puis, ils se montrent de moins en moins saillants en passant par les Dupuyi, Rumanica, Gueretini, subponderosa, Gougetana et Coutagni.

Au point de vue de la taille, la subponderosa est la plus grande, puis arrivent, par rang de grandeur, les Mabilli, ponderosa, Rumanica, Dupuyi, Gueretini, Gougetana, enfin, la Coutagni, la plus petite de toutes.

Comme convexité, la Mabilli vient en tête, puis se succèdent les ponderosa, Rumanica, Dupuyi, subponderosa, Gueretini, Gougetana et Goutagni.

Toutes ces espèces. sont des Acéphales du centre de l'Europe, aimant les eaux profondes, vaseuses, tranquilles et un peu ombragées.

\section{Anodonta Mabilu.}

Grande et belle espèce, très pesante, très ventrue (Jّ4 mill.), à sommets très bombés, volumineux et forte- 
ment proéminents, caractérisée par' une forme oblongueovoïde, à contours (supérieur et inférieur) convexes, surtout l'inférieur qui est arqué-descendant.

Long. $\max . . . \ldots \ldots \ldots \ldots \ldots . . . . .130$ mill.

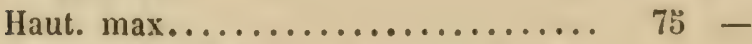

Epaiss. max (à 34 des sommets, à 70 du rostre, à 61 du bord antérieur, à 61 de l'angle postéro-dorsal, à 47 de la base de la perpend.)................

Long. de la crête ligam.-dorsale des sommets à l'angle postéro-dorsal........

Dist. de cet angle au rostre......... 57 -

Corde apico-rostrale............ 100 -

Haut. de la perpendiculaire.......... 75 -

Dist. de cette perpend. au bord antér... 45 -

- du même point de cette perpend. au rostre.....................

- enfin, de la base de la perpend. à l'angle postéro-dorsal........ is -

Coq. à valves à peine bâillantes en avant et en arrière. Bord supérieur légèrement arqué, en suivant une faible direction inclinée. Région antérieure arrondie, largement développée, cependant sensiblement décurrente à sa base. Bord inférieur convexe-descendant, puis remontant à son extrémité vers la partie rostrale. Région postérieure peu allongée, comparativement au développement de la région antérieure et à la grande hauteur relative des valves, terminée par un rostre court, exigu, subaigu, bien qu'arrondi. Entre ce rostre et l'angle postéro-dorsal, le contour descend d'une façon presque rectiligne. 
Sommets volumineux, ventrus-proéminents, comme recourbés dans une direction d'arrière en avant, pourvus de crochets émoussés, et, sillonnés par des striations fines, délicates, serrées et régulières. Arête dorsale, confondue, vers la région ombonale, dans la convexité, se faisant sentir seulement vers l'extré nité dorsale où l'on observe comme deux parties anguleuses, émoussées, divergentes, qui viennent aboutir aux extrémités (supérieure et inférieure) du rostre. Crête dorsale très courte, non développée.

Stries assez fines et émoussées sur la région ventrale, devenant vers les contours de plus en plus fortes, irrégulières et grossières.

Épiderme d'un brun-corné obscur, s'éclaircissant en un ton cendré jaune-rougeâtre sur la région ombonale et se fonçant en une nuance marron de plus en plus accentuée sur la région postérieure, notamment entre le rostre èt l'angle postéro-dorsal. Intérieur d'une belle nacre blanchâtre irisée, non lisse, mais presque entièrement chagrinée, à l'instar de la nacre de certains Unios de Grèce ou de Turquie.

Ligament antéro-interne, lamelleux, très épais, occupant toute la région cardinale et se terminant en dessous de l'autre ligament, à plus de 20 mill. en arrière des crochets. Ligament postérieur robuste, volumineux, saillant, offrant à son extrémité une large lunule qradrangulaire de 11 mill. de longueur.

Charnière forle, épaisse, ondulée. Impressions : cardina.e profonde, en forme de palette; latérale superficielle; palléale bien marquée et comme frangée.

Cette belle espèce, à laquelle j'attribue le nom de notre ami Jules Mabille, habite dans la Loire, près de Tours, où elle a été découverte par le $\mathrm{D}^{\mathrm{r}}$ Rambur. 


\section{Anodunta ponderosa.}

Anodonta ponderosa, C. Pfeiffer, Deutsch. Moll, 11, 182כ, p. 31, pl. IV, f. 3-4 (seulement 1 ).

Cetle Anodonte est fort bien représentée (fig. 3 et 4) dans l'ouvrage de Pfeiffer. C'est une grande espèce ventrue, à valves pesantes, grossièrement striée, d'une teinte oliveâtre uniforme plus ou moins noire. La région postérieure se termine par une large partie rostrale très obtuse, non anguleuse. La hauteur maximum est relativement très grande et dépasse la moitié de la longueur.

La ponderosa est spéciale aux eaux vaseuses, on aux fleuves ou rivières boueuses de toute l'Allemagne : elle a été recueillie aux environs de Pyrmont (C. Pfeiffer), de Dresde (Rossmässler), et de Vienne, en Autriche. Elle vit dans le Danube, d'où je la possède d'un certain nombre de localités, notamment des environs de Giurgewo, en Valachie (Letourneux).

Je rapporte à cetie espèce les ponderosa représentées par Rossmässler (Iconogr. IV, 1836, lig. 282) et par le sieur Drouët (Naiades de France, pl. VI). Seulement, il convient d'éliminer de la synonymie donnée, par ce dernier auteur, à cette Anodonte, les Mytilus incrassatus de Sheppard, Avonensis de Nontagu, etc..., qui sont des formes bien différentes de celle-ci.

Je ne connais pas cet Acéphale en France, bien qu'il ait été signalé dans la Garonne à Agen, aux environs d'Abbe-

1. Les tigures 1 et 2 sont, à mon sens, des jeunes d'une autre espèce. 
ville, de Metz et de Troyes dans l'Aube. Il est probable que ces soi-disants ponderosa sont ou des Dupuyi, ou des subponderosa, ou bien encore d'autres formes voisines qui me sont inconnues. J'ajouterai que je ne puis admettre comme ponderosa l'Anodonte figurée sous ce nom (Moll. France, pl. XVIII, f. 12) par l'abbé Dupuy.

\section{Anodonta rumanica.}

Anodonta rumanica, Letourneux mss.

Ciette belle espèce, recueillie dans le Danube aux environs de Giurgewo, en Valachie, par notre ami le conseiller Letourneux, est au groupe des Ponderosa ce qu'est la stagnalis à celui des Pammegala.

Chez la ponderosa, type, de C. Pfeiffer, les bords (supérieur et inférieur) sont presque parallèles; la région pos. térieure, à peine atténuée à son extrémité, largement obtuse-arrondie, est presque aussi dilatée en hauteur que l'antérieure.

Thez la Rumanica, la région antérieure, bien que légèrement décurrente à sa base (ce qui n'a pas lieu chez la ponderosa), est très dévelop ${ }_{i}$ )ée, en hauteur, comparativement à sa région postérieure qui s'en va en s'atténuant en un rostre assez aigu, dans une direction faiblement descendante. Chez cette Anodonte, la perpendiculaire coïncide avec la hauteur maximum (72 mill.). Chez la ponderosa, ce maximum se trouve reporter en arrière presque au niveau de l'extrémité du ligament postérieur.

Les bords (supérieur et inférieur) sont aussi bien et aussi fortement convexes l'un que l'autre. Le supérieur, notamment, descend jusqu'au rostre, en s'arquant régu- 
lièrement, par suite de l'angle postéro-dorsal non saillant et comme atrophié.

Les sommets, gros, ventrus et proéminents, sont à peine ridés. La crête dorsale est nulle, ainsi que l'angle postéro-dorsal.

Les valves très épaisses, pesantes, d'une leinte sombre plus ou moins noirâtres ou olivâtres, sont très faiblement båillantes en avant et en arrière.

Voici la mersuration de la Rumanica :

Long. $\max . . . . . \ldots \ldots \ldots \ldots \ldots . . .133$ mill.

Haut. $\max . . . \ldots \ldots \ldots \ldots \ldots \ldots . \ldots . \ldots$.

Épaiss. max. (à 35 des sommets, à 80 du rostre, à 51 du bord antér., à 36 de l'angle postéro-dorsal, à $\mathbf{4 1}$ de la base de la perpend.).................

Long. de la crête ligam.-dors. des sommels à l'angle postéru-dorsal.......

Dist. de cet angle au rostre...........

Corde apico-rostrale...............

Haut. de la perpendiculaire......... 72 ?

Dist. de la perpend. au bord antér.... $39 \quad$

- du même point de cette perpend. au rostre............... $\quad 6 \quad-$

- enfin, de la base de la perpend. à l'angle postéro-dorsal....... 73 -

La Rumanica, en un mot, est une pondéreuse très dilatée en avant, et qui, à partir de la perpendiculaire, va en s'amoindrisśant d'une fuçon régulière, pour se terminer par un rostre assez exigu. 


\section{Anodouta subponderosn.}

Anodonta subponderosa, Dupuy, Cat. extr. Galliæ test, $n^{\circ} 29,1849$, et, Moll. France $\left(6^{\mathrm{e}}\right.$ fasc., 1852), p. 607 , pl. XVII, f. 14.

Espèce parfaitement rendue dans l'ouvrage de l'abbé Dupuy. Elle est moins ventrue que les précédentes; ses valves sont moins pesantes; sa région postérieure plus allongée, et, son arête dorsale est un tant soit peu concave vers sa partie moyenne.

Elle a été découverte dans les bassins du Capitany près de Montferrant, dans le Gers, où elle vit en compagnie de la Castropsis de Fagot 1.

Je la connais du Tech, dans les Pyrénées-Orientales où Massot l'avait prise pour la Ventricosa. Je l'ai reçue par. faitement typique d'un ruisseau de la propriété Léon, à Saint-Esprit, près de Bayonne, sous le nom fantaslique de piscinalis var. vetula, que lui avait imposé le très savant Gassies.

Quelques auteur's ont rapporté à cette Anodonte, la ponderosa var. elongata de Picard (Moll. Somme, p. 314, 1840) des environs d'Abbeville. Comme je n'ai pas vu les échantillons de cette localité, je ne cite cette synonymie qu'à titre de renseignement. 


\section{Anodonta Dupuyl.}

Anodonta l)upuyi, Ray et Drouët, Desc. nouv. Anod. in: Rev. zool., p. 32, pl. 1 et 2, 1849.

La Dupuyi a été décrite et bien figurée par Ray et Irrouët, ainsi que par l'abbé Dupuy (Moll. France, $6^{\circ}$ fasc. 1852, p. 606, pl. XVII, f. 3).

Elle vit dans les eaux brourbeuses, tranquilles et ombragées. Le type a été recueilli à Notre-Dame-des-Prés, proche Troyes, ainsi que dans un vivier près de Bar-surAube, et, dans la Marne aux environs de Vitry-le-François. Je connais, en outre, la Dupurji de la Drée (Saône etLoire), de divers étangs près de Metz (Moselle). Ce sont vraisemblablement ces échantillons qui ont été pris pour des ponderosa.

Cette espèce est répandue dans presque toute l'Europe. Je l'ai reçue d'Angleterre, du Danemark, de Pologne (notamment de Ruda, sous le nom erroné de piscinalis). Je sais, de plus, qu'elle vit en Hongrie, en Saxe, en Bavière, en Autriche, etc.

Cette Anodonte est très variable, et, malgré sa grande variabilité, son aspect est si particulier, qu'il est très facıle de reconnaître ses variétés.

\section{Anodonta Gougetana.}

Anodonta Gougetana, Ogérien, Dese. n. esp. Anod. in :

Rev, et mag. zool. 1861, p. 11วّ, pl. III, et, Hist. nat. Jura, III, Zoologie, p. 5ొร้0, fig. 206-208, 1863.

Cette Anodonte qui, ì la rigueur, pourrait être consi- 
dérée comme -une variété minor de la Dupuyi, mérite, néanmoins, d'être distinguée. Je renvoie à la description et aux excellentes figures données par le frère Ogérien pour la connaissance de celte espèce.

La Gougetana habite dans le Jura, les canaux de la saline de Montmorat dans le biez qui passe sous la roue des puits à sel, et, dans la petite rivière du Solan, derrière l'hôpital de Lons-le-Saulnier. Cette espèce aime les eaux profondes, vaseuses et solitaires. On la rencontre toujours abondamment, d'après le dire du frère Ogérien, près des cadavres d'animaux jetés à la rivière.

\section{Anodonta Gueretini.}

Anodonta Gueretini, Servain, mss.

Cette espèce, dédiée au $D^{\mathrm{r}}$ Gueretin d'Angers par notre ami le $D^{r}$ Servn'n, cst une petite pondéreuse à forme tout à fait ovoïde dans une direction légèrement descendante, sans angles saillants, dont les extrémités (antérieure et postérieure) à peu près aussi hautes l'une que l'autre, sont presque également aussi largement arrondies. La convexité, relativement forte, est bien régulière. Les valves épaisses et pesantes, d'une teinte noir-rougeâtre uniforme, sont à peine bâillantes en avant et en arrière.

Long. $\max . . . \ldots \ldots \ldots \ldots \ldots . . . . . .60$ mill.

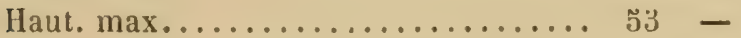

Épaiss. max. (à 18 des sommets, à รัฐ̆ du rostre, à $41 \mathrm{du}$ bord antér., à 26 de l'angle postéro-dorsal, à 38 de la base de la perpendiculaire).......... 32 - 
Long. de la crête ligam.-dors, des sommets à l'angle postero-dorsal....... 38 mill.

Dist. de cet angle au rostre......... 40 -

Corde apico-rostrale............. 72 -

Haut. de la perpendiculaire......... 51 -

Dist. de celte perpend. au bord antér... 30 -

- du même point de cette perpend. au rostre............... 6i3 -

- enfin, de la base de la perpend. à

l'angle postéro-dorsal....... 54 -

Bord supérieur arqué-descendant. Région antérieure bien développée et arrondie. Bord inférieur convexe-descendant, puis remontant par une courbe bien arrondie vers le rostre. Région postérieure dépassant deux fois la longueur de l'antérieure, bien développée en hauteur, diminuant fort peu à son extrémité, qui se trouve terminée par une large partie rostrale bien ronde, sans angles saillants.

Sommets très obtus (toujours excoriés), non proéminents, bien ronds et ne dépassant pas la convexité bien régulière de la région ombonale. Arête dorsale non accusée, se confondant dans la convexité. Crête dorsale presque nulle, non comprimée.

Stries asvez fortes, irrégulières, devenant grossières vers les contours. Épiderme d'un ton uniforme noir-rougeâtre très foncé. Intérieur d'une belle nacre bleuâtre assez mate.

Ligament antéro-interne lamelleux, très épais. Ligament postérieur gros, saillant, avec une grande lunule à son extrémité.

La Gueretini vit dans le Louet, à Juigné-sur-Loire, dans le Maine-et-Loire. 


\section{Anodonta Coutagni.}

Petite Anodonte peu ventrue, allongée en forme de fer de lance dans une direction obliquement descendante, assez bien rostrée, à valves peu épaisses, médiocrement pesantes, à peine bâillantes en avant et en arrière, recouvertes d'un épiderme d'une teinte uniforme cendré-olivâtre, s'éclaircissant vers les sommets et se fonçant quelquefois en vert à la région postérieure.

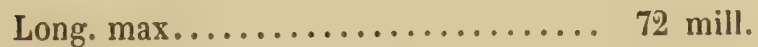

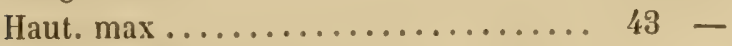

Épaiss. max. (à 21 des sommets, à 40 du rostre, à $32 \mathrm{du}$ bord antér., à 19 de l'angle postéro-dorsal, à 22 de la base de la perpendiculaire.).......... 22 -

Long. de la crête ligam.-dors. des somm. à l'angle postéro-dorsal.......... 27 -

Dist. de cet angle au rostre.......... 40 -

Corde apico.rostrale.............6. 61 -

Haut. de la perpendiculaire......... 35 -

Dist. de la perpend. au bord antér..... 19 -

- du même point de cette perpend. au rostre............... 54 -

- enfin, de la base de la perpend. à l'angle postéro-dorsal....... 41 -

Bord supérieur peu allongé, presque rectiligne jusqu'i l'angle postéro-dorsal, puis descendant sur le rostre tout en conservant une légère courbure. Région antérieure exiguë, arrondie et fortement décurrente à la base. Bord 


\section{$-206-$}

inférieur descendant presque en ligne droite jusqu'à 27 mill. en arrière de la perpendiculaire, pour remunter par une courbe vers la partie rostrale. Région postérieure très allongée, allant en se développant dans le sens de la hauteur jusqu'au niveau d'une verticale passant par l'extrémité du ligament postérieur, pour diminuer ensuite jusqu'à un rostre arrondi plus ou moins accentué, suivant les échantillons.

Sommets régulièrement convexes, non proéminents, à crochets très aigus et sillonnés par des rides assez délicates. Arête dorsale nulle, offrant quelquefois vers l'extrémité postérieure, deux zones subanguleuses, divergentes, venant aboutir en dessus et en dessous du rostre, qui, dans ce cas, paraît faiblement troncatulé. Crête dorsale peu développée, un tant soit peu comprimée vers l'angle postéro-dorsal.

Stries lisses sur la région ventrale, devenant de plus en plus fortes et grossières vers les contours. Epiderme d'un cendré-olivâtre, passant à un ton pâle vers les sommets, oủ il devient d'une nuance jaunacée-rougeâtre. Sur certains échantillons, l'épiderme se colore en vert à la partie postérieure. Intérieur d'une nacre d'une teinte mate bleuacée.

Ligament antéro-interne lamelleux, très mince. Ligament postérieur volumineux, saillant, terminé par une lunule triangulaire. Charnière filiforme, présentant parfois, juste au-dessous des crochets, ure iamelle tuberculeuse.

Cette Anodonte, très caractérisée, qui est pour le groupe des Ponderosa ce qu'est la Rayi pour celui des Anatina, a été recueillie par l'ingénieur G. Coutagne, auquel je la dédie, dans l'Albane (Gôte-d'Or), affluent de la Bèze, rivière qui se jette dans la Saône. 


\section{0 ROSGI ÄSTERIANA}

Les Anodontes de ce groupe préfèrent les rivières, les ruisseaux, en un mot, les eaux vives et courantes à fond d'argile ou de graviers. Elles ont un tout autre genre de vie que les Ponderosa, dont elles sont, néanmoins, un peu voisines d'aspect et de caractères. Elles ont, en effet, jusqu'à un certain point, des valves relativement épaisses, et, toutes sont d'une couleur sombre 1 .

\section{Anodonta IRossmåiss leriana}

Anodonta Rossmässleriana, Dupuy, Essai Moll. Gers, p. 74, 1843, et Moll. France ( $6^{\mathrm{e}}$ fasc. 1852), p. 608, pl. XVIII, f. 14.

Cette espice, très bien représentée par l'abbé Dupuy, vit dans les petites rivières du sud-ouest de la France : le Gers, la Baise, la Gimonne, etc. (Dupuy). Je la connais encore de l'Ossun, à Tarbes, dans les Hautes Pyrénées; du canal entre Loir et Cher, près de Tours (Rambur); de la Marne, à Langres; enfin, du petit ruisselet des Vignes à Amances près de Vendeuvre-sur-Barse, dans l'Aube.

1. Je suis assez tenté de comprendre dans ce groupe la Ponderosa de l'abbé Dupuy (Moll. France, pl. XVIII, f. 12), qui, à mon sens, bien qu'elle me soit inconnue, ne me semble pas pouvoir être rapportée \& la Pondercsa de C. Pfeiffer. 


\section{Anodonta laxats.}

Anodonta luxata, Held, in : Isis, IV. 1837, p. 305.

- - Küster, Anod. (in : $2^{\mathrm{m} \theta}$ édit. Chemnitz.), p. 9 , pl. III, f. 1, 1852 .

Assez parfaitement rendue par Küster, bien que très mal coloriée, cette Anodonte paraît commune en Allemagne, notamment en Bavière aux environs de Passau. Je la possède de Damhnussoën en Danemark, d'où Mörch me l'a adressée sous le nom de Cellensis, Var. 3, minor 1. Je connais en France, du Serain à Monetau, près d'Auxerre, dans l'Yonne (Mabille), et de la Marne à Jaulgonne, dans l'Aisne (Lallemant).

\section{Anodonta inornata.}

Anodonta inornata, Küster Anod. (in : 2e édit. Chemnitz), p. 42, pl. III, f. 6, 1852.

Anodonta radiata, var. f. A. inornata. Mörch, Syn. Moll.

Danioe, p. 85, 1864.

Espèce répandue en Allemagne, où elle a été découverte dans le Regnitz, près d'lirlangen en Bavière. Mörch me l'a envoyée d'Alberslung en Danemark. Je l'ai reconnue dans notre pays, du Tech dans les Pyrénces-Orientales (Fagot), de l'Ain à Brainans près de Poligny, dans le Jura (Mabille), de la Loire à Tours (Rambur); enfin de l'Yvette à Orsay, près de Paris (Coutagne).

1. Voir Mörch, вyn. Moll. Daniœ, p. 85, 1864. 
Les échantillons de celte dernière localité sont plus petits que le type, qui, entre parenthèses, est assez mal rendı dans l'ouvrage de Küster. Ils peuvent constituer une variété minor.

\section{Anodonta Sondermanni.}

Anodonta Sondermanni, Küster, Anod. (in : $2^{\circ}$ édit. Chemnitz), p. 54, pl. XIII, f. 4, 1852.

Cet Acéphale provient du Danube près de Regensburg (Ratisbonne) en Bavière. La figure, qu'a donné Küster de cette espèce, est suffisante pour la connaissance de la Sondermanni.

\section{Anodonta vilssoni.}

Anodonta Nilssoni, Küster, Anod. (in : $2^{\circ}$ édil. Chemnitz), p. 61, pl. XVIII, f. 2, 1852.

Du Regnitz, près d'Erlangen, en Bavière.

J'ai reçu en communication une Anodonte d'Upland, en Suède, étiquetée anatina par Westerlund, qui n'était autre chose que la Nilssoni.

\section{20 IBHOTHANA}

Les Brotiana ont un aspect sombre, d'une teinte uniforme marron ou oliveâttre, ou bien d'une nuance cendréerougeâtre, s'éclaircissant sur la région de la plus grande IIAI 1881. 
convexité. Les striations sont rudes et grossières vers les contours. Le bord des valves, depuis l'angle postérodorsal jusqu'au bord postéro-palléal, très épaissi, souvent rongé, paraît comme maladif.

Les espèces de ce groupe, toutes particulières à la Suisse, peuvent se répartir en deux séries : $1^{\circ}$ en coq. de forme légèrement subtrigone (Broti, tumida); 20 en coq. de forme obtuse-allongée (Humberti, Charpentieri).

\section{Anodonta Broti.}

Cette Anodonte a été trouvée par Brot, auquel je la dédie, dans le lac de Baldegg, en Suisse, et, adressée, sous l'appellation erronée d'anatina, à notre ami Deshayes, duquel je la tiens.

C'est une forme assez comprimée, ovalaire-subtrigonale, à région antérieure très exiguë, décurrente à la base, tandis que la postérieure très développée en hauteur ( 61 mill.) et en longueur ( 73 mill.), dans une direction obliquement descendante, offre un contour subquadrangulaire (1 angle inférieur à l'extrémité du bord palléal; 2 autres au rostre, enfin 1 dernier, qui n’est autre chose que l'angle postéro-dorsal). Le lest du contour postérieur, toujours très épaissi, est comme bordé. Les valves sont fortement bâillantes en avant et fort peu en arrière.

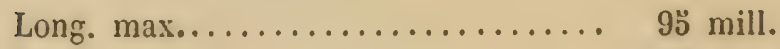

Haut. $\max . . . . . . . . . . . . . . . .6 .61$ -

Épaiss. $\max$ (à 27 des sommets; à 57 du rostre; à 41 du bord antér.; à 29 de 
l'angle postéro-dorsal; à $: 36$ de la base de la perpend.)...............

29) mill.

Long. de la crête ligam.-dors., des sommets à l'angle postéro-dorsal....... 14 -

Distance de cet angle au rostre........

Corde apico-rostrale............. 84 -

IIaut. de la perpendiculaire......... $8:-$

Dist. de cette perpend, au bord antér... 2/4 -

- du même point de cette perpend. au roitre................... 73 -

- enfin, de la base de la perpend. à l'angle postéro-dorsal.............

$62-$

Bord supérieur relativement assez court, presque rectiligne ou plutôt un tant soit peu convexe jusqu'à l'angle postéro-dorsal, pour, de là, descentre en droite ligne sur le rostre. Région antérieure exiguẻ, arrondie, très décurrente à la base. Bord inférieur convexe-descendant jusqu'à 36 mill. en arrière de la perpendiculaire, puis prenant (en donnant lieu à un angle) une direction as. cendante, presque rectiligne jusqu'à la partie inféro-anguleuse du rostre. Région postérieure plus de trois fois plus longue que l'antérieure, très haute $(61$ mill.), terminée par un large rostre troncatulé.

Sommets très antérieurs, obtus, à peine proéminents (crochets émoussés) et sillonnés par de fortes rides tremblotées. Arête dorsale faiblement accentuée seulement à la région ombonale, confondue ensuite dans la convexité et ne s'accusant que par la présence de deux zonules noirâtres, divergentes, qui aboutissent au rostre. Crête dorsale relativement développée et assez comprimée.

Stries délicates sur la région la plus convexe, puis de-: 
venant de plus en plus fortes, saillantes, mème grossières et feuilletées vers les contours. Épiderme d'un cendréjaunacé en avant, s'éclaircissant en une nuance jaunebrique sur la partie ventrale; enfin, prenant sur toute la région postérieure un ton marron, passant au marronviolet vers la crête dorsale. Intérieur d'une nacre blanchebleuacée.

Ligament antéro-interne très volumineux. Ligament postérieur saillant, robuste, terminé par une longue lunule subquadrangulaire. Charnière ondulée, avec une petite éminence tuberculeuse près des crochets.

Sans compter le lac de Baldegg, où a été trouvé le type, cette Anodonte a encore été recueillie, dans le Rosenbach près d'Ebikon, ainsi que dans le lac de Neuchâtel (Coutagne).

\section{Anodonta Iamida.}

Anodonta tumida, Küster, Anod. (in : $2^{\circ}$ édit. de Chem. nilz), p. 48, pl.XI, f. 1-2, 1852 .

Je conserve à cette espèce le nom de tumida que lui a imposé Küster, bien qu'il existe une Anod. tumida, du Danube (près de Petronell), signalće, sans caractères, par Fitzinger en 1833 (Syst. Verz. Oesterr. Weichth., p. 121).

Je rapporte à cet Acéphale, la Picteliana var. rostrata de Brot (Naïad. Léman., pl. ViII, f. 2), que cet auteur indique du lac de Genève, aux environs de Villeneuve, où cette Anodonte vit en compagnie de la véritable Pictetiana. La tumida, décrite par Küster, a aussi été trouvée dans le lac de Genève, seulement entre Morges et Lausanne. 


\section{$-213-$}

Cette espèce est voisine, comme forme, de la Broti, dont elle diffère par sa plus grande convexité; par sa ré. gion postérieure à contours non anguleux, à rostre non troncatulé ; par ses sommets un peu moins antérieurs, etc.

\section{Anodonta Inmbert.}

Cet Acéphale est remarquable par une convexité très prononcée de l'arête dorsale, convexité qui se fait sentir presque sans atténuation jusqu'au rostre, où elle se termine assez brusquement.

L'Humberti a une forme allongée très obtuse dans une direction légèrement descendante à partir des sommets, avec une légère sinuosité à son bord palléal. Les valves, assez épaisses, sont fortement bâillantes en avant.

Long. $\max . . . \ldots \ldots \ldots \ldots \ldots . . .69$ mill.

Haut. $\max . . \ldots \ldots \ldots \ldots \ldots \ldots . . . .65$

Épaiss. max (à 31 des sommets; - à 47 du rostre; à 49 du bord antér.; à 23 de l'angle postéro-dorsal; à 3 de la base de la perpend.)..............

Long. de la crête ligamento-dorsale, des sommets à l'angle postéro-dorsal.....

Dist. de cet angle au rostre...........

Corde apico-rostrale............. 79 -

Haut. de la perpendiculaire......... 50 -

Dist. de cette perpend. au bord antér... 28 -

- du même point de cette perpend. au rostre......................

- enfin, de la base de la perpendiculaire à l'angle postéro-dorsal...... 


\section{$-214-$}

D'après ces mesures, l'on voit que le point de la plus grande épaisseur, assez voisin de l'angle postéro-dorsal et assez distant des sommets et de la base de la perpendiculaire, est plus rapproché du rostre que du:bord antérieur.

Bord supérieur un tant soit peu arqué jusqu'à l'angle postéro-dorsal, puis descendant sur le rostre par un contour légèrement convexe. Région antérieure assez grrande, bien arrondie, à peine décurrente à la base. Bord inférieur faiblement convexe-descendant, oflrant à 17 mill. de la perpendiculaire une faible sinuo:ité concave, et un peu plus loin, à 38 mill., une dilatation subarrondie pour de lid remonter par une courbe régulière vers la partie rostrale. Région postéricure, plus de deux fois plus longue que l'antérieure, allongće dans une direction descendante et terminée par un lurge rostre très obtus et arrondi, ayant une tendance à regarder en bas.

Sommets assi $z$ ventrus, médiocrement proéminents, à crochets émoùssés, et sillonnés par des rides serrées peu accentuées. Arête dursale très convexe, conservant sa convexité presque jusqu'au rostre, et présentant une inclinaison très rapide sur la crête dorsale, qui est peu développée et un tant soil peu comprimée vers l'angrle postéro-dorsal.

Stries médiocres, rugueuses, trè̀ grossières et feuilletées vers les contours. Epiderme d'un brun-marron, s'éclaircissant en rouge brique sur toute la région de l'arête dorsale. Intérieur d'une nacre blanchâtre avec des taches livides.

Ligament antéro-interne très épais. Ligament postérieur fort, saillant. Lunule tantôt pyriforme, tantôt triangulaire. Charnière aveć une éminence tuberculeusé allongée sur la région cardinale. 
Cette espèce, à laquelle j'attribue le nom d'Aloïs Humbert de Genève, auteur d'un grand nombre de mémoires malacologiques, vit dans l'Aa, au sortir du lae de Baldegg. Brot a envoyé à notre regretté Deshayes, cette Anodonte sous l'appellation erronée d'anatina var. decurvala.

\section{Anodonta Charpentieri.}

Anodonta Charpentieri. Küster, Anod. (in : Q édit. Chemnitz), p. 49, pl. XI, f. $3-4,1852$.

Celte bivalve, dédiće à notre ancien ami, feu Jean Charpentier, de Bex, habite le lac Morat, près de Faouy, en Suisse. Elle est suffisamment bien repré:entée dans l'ouvrage de Küster.

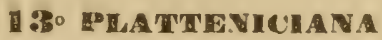

Toutes ces Anodontes sont spéciales au grand lac Balaton, véritable petite mer intérieure de la Hongrie, où elles ont été découvertes par notre ami le docteur Servain. Toutes possèdent une impression palléale frangée et sont teintées d'une nuance uniforme cornée plus ou moins foncée, s'éclaircissant sur la réşion ventrale, sauf l'aquatica, en partie olivâtre.

Chez ces espèces, à l'exception dı la Tissoti, l'arête dorsale, très bombée, est limitée supérieurement par un sillon plus ou moins accentué descendant des sommets au rostre. Les valves présentent un seul bâillement peu ouvert, sauf chez la Tissoti, où l'on en remarque deux considérables et l'aquatica, où ils sont peu prononcés. Les 
stries, généralement fines, émoussées, toujours régulières, même vers les contours, le sont moins chez les Balatonica et Tihanyca. La convexité accentuée sur la région de l'arête dorsale, est, dans la plupart des cas, presque médiane.

Ces Acéphales peuvent se répartir en deux séries : $1^{\circ}$ en coq. oblongue, à partie rostrale plus ou moins prononcée (Plaltenica, Balatonica et Tihanyca. Cette dernière est la plus rostrée); $2^{\circ}$ en coq. ovalaire, à rostre très obtusarrondi (Tissoti, Hydatina), ou, ovalaire-subarrondie (aquatica).

Chez les Anodontes de la première série, on constate, sur la région latérale de la charnière, la présence d'une lamelle. Chez celles de la deuxième, on ne remar que aucune éminence, sauf pourtant chez la Tissoti, où il y a un semblant de sillon lamelliforme.

\section{Anodonta Plattenica}

Anodonta Plattenica, Servain, Hist. malac. lac Balaton, 1881. (Sous presse.)

Espèce d'une forme oblongue un tant soit peu allongée dans une direction faiblement descendante, sans angles saillants, très haute ( 59 mill.) pour sa longueur (9ٌ mill.), pourvue d'une partie rostrale assez accentuée et offrant une convexité maximuin (36 mill.) également distante du rostre et du bord antérieur, convexité, du reste, bien régulière, sauf entre l'arète dorsale et la crête ligamentodorsale, où elle descend rapidement. Valves assez épaisses, malgré tout, trarsparentes, d'une teinte cornée-jaunâtre uniforme, s'éclaircissant sur la région ventrale ; en- 
fin, ne présentant qu'un seul bâillement antérieur, assez ouvert.

Bords du lac Balaton à Füredi, Fürdö et Palaznak.

\section{Anodonta Balatoniea.}

Anodonta Balatonica, Servain, Hist. malac. Balat., 1881. (Sous presse.)

Chez cette espèce, un peu moins ventrue et d'une teinte cornée plus foncée, la partie postérieure, moins large, est plus descendante; le rostre, plus allongé, est un tant soit peu subanguleux, avec une tendance à devenir subaigu ; l'arête dorsale descend sur la partie supéro-rostrale d'une façon plus rectiligne; les sommets, un peu plus antérieurs, sont un peu moins ventrus; la convexité maximum est plus rapprochée du bord antérieur ( 43 mill.) que du rostre ( 51 mill.); la plus grande hauteur ( 56 mill.) se trouve moins éloignée de la perpendiculaire, à 21 mill. seulement, tandis que, chez la Plattenica, elle s'écarle de 31 mill. en arrière de cette ligne. Il résulte de là que, la plus grande hauteur de la Balatonica étant plus rapprochée de la perpendiculaire, le bord inférieur n'offre pas à son extrémité de renflement convexe analogue à celui qui caractérise ce bord chez la Plattenica.

En somme, comme forme, la Balalonica, par suite du non renflement de l'extrémité de son bord inférieur, et de la hauteur moins grande de sa région postérieure, qui se termine en un rostre assez aigu, a une apparence assez trigonale. Ses valves, comme celles de la Plattenica, sont assez épaisses, et elles n'offrent qu'un seul bâillement antérieur, seulement moins ouvert.

Bords du iac Balaton (Servain). 


\section{Anodonta Tihanyca.}

Anodonta Tihanyca, Servain, Hist. malac. Balat., 1881. (Sous presse.)

Coq. caraclérisée par une partie antérieure bien ronde et très développée, et par une légion postérieure obliquement descendante, s'étranglant presque subitement à sa moitié postérieure, se prolongeant ensuite en un rostre un peu en forme de bec de corbin. Ce rostre, assez aigu, est légèrement troncatulé. La plus grande hauteur (う3 millimètres $1 / 2$ ), chez cette espèce, est très rapprochée (à 8 mill.) de la perpendiculaire. L'épaisseur maximum (33 mill.) est presque centrale; enfin, la convexité est moins régulièrement répartie que chez les deux espèces précédentes.

L'étranglement de la région postérieure donne à la Tihanyca un aspect tout à fait particulier; elle a l'air d'être tout en avant. Malgré ce prolongement rostral un peu insolite, cette espèce n'appartient point au groupe de Ia rostrata, comme on pourrait le penser. Elle rentre bien dans celui de la Plattenica, dont elle otfre tous les autres caractères. Ses valves, en efỉet, un peu moins épaisses que celles des précédentes, oflrent un épiderme semblable à celui des Plattenica et Balatonica. Le bâillement antérieur est également analogue.

Rives de la presqu'ile Tihany, dans le lac Balaton (Servain). 


\section{Anodonta Tissoti.}

Anodonta Tissoti, Servain, Hist. malac. Balat., 1.8\$1. (Sous presse.)

Espèce de forme ovale, sans angles saillants, à convexité médiocre, bien régulière, dont le point maximum (30 mill.) est à égale distance du rostre et du bord antérieur. Arête dorsale ni accentuée, ni bombée, comme chez les précédentes, mais nulle et confondue dans la convexité. Sommets excessivement oblus, presque comprimés. Vatres toujours d'une teinte cornée uniforme, plus claire sur la région ventrale, dilférant, en outre, des précédentes, en ce sens qu'elles offrent deux forts bâillements : $1^{\circ}$ un entre l'angle postéro-dorsal et le rostre; $2^{\circ}$ un aulre, très grand et très ouvert, s'étendant sur tout le contour du ligament antéro-interne à la partie rostrale.

Bords du lac Balaton, à Sio Fok et vers les bains de Füred (Servain).

\section{Anodonta Fydatina.}

Anodonta Hydatina, Servain, Hist. malac. Balat., 1881. (Sous presse.)

Petite Anodonte remarquable par sa forme ovalaire, très haute (48 mill.) pour sa longueur ( 74 mill.), à région postérieure bien rostrée. Le maximum de l'épaisseur (30 mill.), peu distant des sommets (22 mill.) et de l'angle postéro-dorsal ( 17 mill.), est plus rapproché du rostre 37 mill.) que du bord antérieur (40 mill.). Chez l'Hyda- 
tina, l'arête dorsale est très convexe, ainsi que toute la région ombonale; les sommets sont plus médians que ceux des espèces précédentes; la région postérieure ( 49 mill.) n'atteint pas deux fois l'ampleur de l'antérieure (27 mili.); les valves sont à peine bâillantes en avant; l'épiderme, enfin, d'un brun corné, plus clair sur la partie ventrale, est très brillant, lisse ou seulement sillonné de stries concentriqques très émoussées et bien régulières.

Lac Balaton, près Füred, Advari, etc. (Servain).

\section{Anodonta aquatica.}

Anodonta aquatica, Servain, Hist. malac. Balat., 1881. (Sous presse.)

Celte espèce, la plus petite des Platteniciana, est remarquable par son peu de longueur (6气 mill.) comparée à sa hauteur (46 mill.); aussi est-elle ovalaire-subarrondie. Ses valves, un tant soit peu bâillantes en avant et en arrière, sont minces, brillantes, d'une teinte olivâtre claire, sur la région de la crête dorsale où la coloration passe à un ton cornć-brunâtre. La convexité maximum, chez l'aquatica, est presque médiane; son arête dorsale est fortement bombće; enfin, sa crête dorsale, notablement comprimée; est relativement très grande et bien plus développée que celle de toutes les autres espèces de ce groupe. Rives du lac Balaton, à Sio Fok, à Füred, etc. (Servain).

\section{H 4 DEPRESTINA}

Les Depressiana sont d'assez grandes espèces (la Browni a 156 mill. de longueur sur 90 mill. de hauteur), remar- 
quables par leurs valves aplaties et par leurs sommets tout à fait comprimés. Elles ressemblent à de jeunes Anodontes, bien qu'elles soient adultes.

Chez les espèces de ce groupe, le rostre est largement obtus, plus ou moins bien arrondi; l'arête dorsale est nulle; la crête est comprimée; le ligament postérieur, presque entièrement recouvert; le bord supérieur, constamment subrectiligne jusqu'à l'angle postéro-dorsal, tandis que le bord palléal est toujours plus ou moins convexe.

Les valves sont généralement brillantes, sans bâillements (Sturmi), ou à un seul bâillement peu ourert, tantôt en avant (Browni, Lutetiana), tantôt en arrière (depressa).

\section{Anodonta depressa.}

Anodonta depressa, Schmidl, Conch. Krain, p. 27, 1848. (Anodonta glabra ${ }^{1}$, de Ziegler, teste Schmidı.)

Je rapporte sous ce nom, bien que je ne connaisse pas le lype de Schmidt, qui n’a été ni décrit ni figuré, une espèce d'Anodonte de Carniole, que j'ai reçu plusieurs fois sous cette appellation.

C'est une grande bivalve (long 115 mill.), très haute ( 68 mill.) pour sa taille, et rélativement épaisse (ép. max. 29 mill.). Sa région postérieure égale près de deux fois et demi la région antérieure. Ses sommets très obtus, tout à fait comprimés, sont à peine sillonnés de rides délicates. Son bord supérieur rectiligne jusqu'à l'angle postéro-

1. Xion, Anod. glabra de Villa, espèce du groupe des Westerlundiana. 
dorsal descend ensuile sur le rostre par un contour lígèrement concave, ce qui donne à la partic rostrale, qui est assez aizuë, une apparence lígèrement ascendante. Le bord inférieur, d'abort bien convexe jusqu'à la base de la perpendiculaire, se poursuit ensuite, sur quelques échantillons, d'une fingon un peu rectiligne, pour remonter vers son extrémité par une courhe accentuce. L'arète dorsale est nulle. La crête, assez développée, est comprimée vers l'angle postéro-dorsal, qui est très obtus. Les stries sont assez grossières et l'épiderme oflre une teinte verdatre, se fonçant vers les contours et passant au cendré.jaunâtre ver's la région ombonale.

La depressa habite dans les lacs de la Carniole et de la Carinthie, notamment dans ceux des environs de Klagenfurt.

\section{Anodonis Erowni.}

Cette grande (long. max. 15is, haut, max. 90 mill.) et magnifique Anodonte aplatie, à laquelle j'attribue le nom du cap. Thomas Brown, a été admirablement représentée par cet atuteur (pl. XIII), dans ses a Illustrations of the land and fresh water conchology of great Britain and Ireland, $184 \%$. $v$ Cette figure sullit amplement di la connaissance do cette espèce, que thomas Brown avait confondue (p. 101) avec l'anatina, qui n'a pas le moindre rapport avec elle.

Cet Acéphale vit aux environs de Prestwick carr, dans le comté de Northumberland, en Angleterre. 


\section{Anodowata sturni.}

Mytilus anatinus (pars), Sturm, Deutsch. fauna, $1^{\text {er }}$ fasc. 1803 (sans parination), pl. 1.

Gette espèce, également représentée sous l'appellation erronée d'analina, est une Anodonte très aplatie, de forme oblongue, à sommets comme écrasés. Elle vit en Bavière, notamment aux environs de Nuremberg, ainsi qu'en Allemagne, aux environs de Gassel, où C. Pfeiller (Nat. deutsch. moll., I, p. 113, pl. VI, f. 3, 1821), l'a signalée sous le nom d'intermedia de Lamarck. Jo l'ai reçue du Danemark, d’où clle m'a été adressée par Mörch, sous le nom d'Anod. Cellensis, var. B. intermedia 1. (Voir Mörck, Syn. moll. Daniac, p. 85, 1864.)

Je la connais, en France, des environs de Cirret, dans les Pyrénées-Orientales; du Rhône, près d’Arles; du canal du Midi, à Villefranche-Lauraguais, près de T'oulouse (Fagot); enfin, de Neuilly-sur-Saône, dans la Côte-d'Or (Locard). Les échantillons de Neuilly sont un peu plus bombés que ceux des autres localités.

\section{anodonta tuterians.}

Anodonta Lutetiana, Mabille, mss.

Long. $\max . \ldots \ldots \ldots \ldots \ldots \ldots \ldots . . . . .69$ mill.

IIaut. $\max . \ldots \ldots \ldots \ldots \ldots \ldots \ldots$ in -

Épaiss. max. (à 24 des sommets; à 32

1. L'Anod. inlermedia de Lamarcls est une forme différento dir groupe des Spengloriana. 
du rostre; à 44 du bord antérieur; à 28 de l'angle postéro-dorsal, à 34 de la base de la perpen 1.).............

Long. de la crête ligamento-dorsale, des sommets à l'angle postéro-dorsal....

Distance de cet angle au rostre........

Corde apico-rostrale..............

Hauteur de la perpendiculaire.........

Distance de cette perpend. au bord antér.

- du mème point de cette perpend. au rostre................

- enfin, de la base de la perpend. à l'angle postéro-dorsal.....

Coq. oblongue, à valves très légères, bien qu'assez épaisses, non bâillantes en arrière, offrant seulement un très faible bâillement en avant.

Bord supérieur presque rectiligne jusqu'à l'angle postéro-dorsal, puis descendant sur le rostre, tout en présentant une très faible convexité. Région antérieure développée, arrondie, un tant soit peu décurrente à sa base. Bord inférieur bien arqué-convexe, à peine descendant, remontant par une courbe bien ronde à son extrémité. Région postérieure oblongue-allongée (un peu plus de deux fois plus longue que l'antérieure), terminée par un large rostre très obtus-arrondi.

Sommets complètement comprimés-aplatis, non saillants (crochets très aigus) et sillonnés par de petites rides ondulées. Arête dorsale nulle, confondue dans la convexité. Crête dorsale assez comprimée.

Stries très fines (obsolètes sur la région ventrale), derenant plus fortes et plus espacées vers les contours et un tant soit peu feuilletées sur la crête dorsale. 
Épiderme brillant, d'un ton opale livido-olivâtre, passant au noir sur la région postérieure, qui est presque toujours encroûtée de calcaire. Intérieur d'une nuance bleuacée pâle, faiblement orangée vers les sommets.

Ligament antéro-interne très délié. Ligament postérieur mince, presqu'entièrement symphynoté. Lunule longue, subquadrangulaire. Charnière légèrement ondulée, à régions : cardinale nulle, latérale relativement épaisse.

Abondante dans la Bièvre à Arcueil, près Paris, (Mabille.).

\section{GO ROSTRATIANA}

Les différentes espèces de ce groupe sont des formes remarquables par le prolongement de leur partie postérieure, terminée par un rostre très accusé, tantôt horizontal ou descendant, tantôt ascendant. Cette partie postérieure est trois fois, ou même quatre fois, plus longue que l'antérieure. Les valves sont minces, assez fragiles, généralement bâillantes (chez quelques formes, les bâillements sont fort considérables); l'épiderme, ordinairement d'une teinte presque uniforme ochracée ou jaunacéc plus ou moins rougeâtre ou brunâtre, voire même quelquefois violacée, est la plupart du temps assez terne (presque toujours encrassée de calcaire) et sillonnée de stries grossic̀res, feuilletées vers les contours. Les sommets sont comprimés, non saillants; la crète dorsale assez amincie; l'arête, généralement bien bombée, est, soit rectiligne, soit concave.

Chez les Rostratiana, le bord supérieur, des sommets à l'angle postéro-dorsal, est toujours rectiligne ou faiblement arqué ; la région antérieure tris exiguë, est plus ou I'A. 1881. 
moins décurrente à la base; enfin, le bord inférieur est, presque dans tous les cas, faiblement convexe-descendant. Il résulte de ces caractères que la région postérieure s'allonge dans une direction oblique plus ou moins inclince, et, que les stries, en accusant la forme des contours, offrent une très grande excentricilé.

La convexité, chez les Anodontes de ce groupe, n'est pas régulièrement répartie; souvent, c'est la région ombonale qui paraît la plus saillante; souvent, c'est la région de l'arête dorsale. Les autres régions sont plus ou moins convexes ou comprimées, voire même aplaties, suivant les espèces. Il n'y a pas, en un mot, de convexité bien régulière, c'est-à-dire de convexité dont la courbe, des sommets au bord palléal ou du bord antérieur au postérieur, s'accuse d'une façon normalement arquée, comme celle que l'on remarque chez l'anserirostris, des groupe des Cygnœana, que certains auteurs veulent à toute force placer parmi les rostrata, parce qu'elle est pourvue d'une partie rostrale assez prononcée.

Cette anserirostris a tous les caractères d'une cygncea ou d'une oblonga, avec une arête dorsale un tant soit peu concave et par cela même légèrement remontante à son extrémité. Sa partie postérieure, non obliquement descendante, comme celle des Rostratiana, est presque horizontale; ses striations n'offrent pas le même dégré ni le même mode d'excentricité : sa convexité est régulière, etc. La figure 1 de la planche $\mathrm{XI}^{\mathrm{a}}$ des Anodontes de Kuster est excellente et rend bien les signes distinctifs de cette espèce. La figure 3 de la planche $\mathrm{X}$ du même ouvrage est bien inférieure à celle que je viens de citer.

Les Rostratiana se séparent aisément en deux séries :

$1^{\circ}$ En espèces possédant une arête dorsale descendant en 
liyne droite des sommets au rostre, et offrant une partie rostrale droite ou faiblement descendante, voire même recourbce (rostrata, diminuta, capitata, Sedakowi, Sebinensis et limpida);

$2^{\circ}$ En espèces pourvue d'une arête dorsale plus ou moins concave, avec une partie rostrale toujours ascendante (Blanci, helvetica, lacustrina).

Toutes ces Anodontes vivent dans les lacs, les étangs, les viviers à fond de gravier et n.on de vase.

\section{Anodonta rostrata.}

Anodonta rostrata, Kokeil, in : Rossmässler, Iconog. IV, 1836, p. 25, fig. 2841 .

Il faut rapporter à la rostrata :

$1^{\circ}$ La var. XI (rostrata) de la planche XVI (figure $1^{\text {ere }}$ seulement) des Illustrations conch. Great-Britain de Thomas Brown;

2० L'Anodonta elongata de Stentz, mss., in : Potiez et Michaud (Gall. moll. Douai, II p. 141, pl. LV, f. 1, 1844), forme de Carniole \& un tant soit peu moins large, peutêtre moins comprimée, de 100 mill. de long., 50 mill. de haut., et 30 mill. d'épaisseur, » au dire de Potiez et Michaud. Or, ces chiffres correspondent presque exactement à ceux de la mensuration de la rostrata type, figurée par Rossmässler.

Il convient au contraire de rejeter :

$1^{\circ}$ L'Anodonta rostrata de Held (in : Isis IV, 1836, p. 280)

1. La figure 737 de l'Iconographie représente la diminuta de Clessin. 
et de Held. in : Küster (Anod. in : $2^{\circ}$ édit. Chemnitz, p. 14, pl. IV, f. 2, 1852).

La même année que Rossmässler publiait, dans son Ico. nographie, la rostrata de Fiokieil, Held en publiait également une de Kokieil dans l'Isis, espèce que Küster reproduisait en 1852 dans son genre Anodonta. Or, cette rostrata éditée par Held, puis par Küster, n'est pas celle de Kiokeil publiée par Rossmässler. La rostrata (Kokeil) de Held et Küster est une forme bien voisine de l'oblonga de Millet, du groupe des Cygnoeana. Je conserve donc le no:n de rostrata à l'espèce représentée (fig. 284) par Rossmässler.

$2^{\circ}$ L'Anodonta rostrata de Drouët, représentée (pl. V, f. 2) dans les Naïades de France (olim., Études sur les Anodontes de l'Aube). Gelte rostrata est la Jourdheuili de Ray, espèce qui n'a pas le moindre rapport avec la rostrata de Rossmässler. le $S^{r}$ Drouët rapporte (Anod. Aube, $2^{\mathrm{e}}$ art., p. 1 ' et $7^{\circ}$ art., p. 17) à sa soi-disant rostrata, sans compter les latissima de Kokeil, confervigera de Schlütter, recurvirostris de Küster, parce que je ne les connais pas ${ }^{1}$, les depressa de Schmidt, anserirostris et Charpentieri de Küster, et enfin, la Pictetiana de Mortillet. Il est difficile de faire un plus complet amalgame. La Pictetiana comprend quatre formes de quatre groupes différents. La Charpenticri est une espèce particulière et distincte des quatre formes qui précèdent; il en est de même de l'anserirostris qui, malgré sa partie rostrale un peu accentuée, appartient par tous ses autres caractères au groupe des Cygnoeana. En somme, M. Drouët a confundu au moins 7 espèces de 6 groupes distincts.

$3^{\circ}$ L'Anodonta piscinalis var. rostrata de Mocquin-Tandon

1. Toutes ces Anodontes sont des espèces inédites. 
(Moll. France, II, p. 561, pl. XLVI, f. 5, 185๊ ) signalée de Saint-Julien, près de Troyes. La rostrata de Saint-Julien est la Jourdheuili de Ray, et la figure 5 (pl. XLVI), loin de représenter la soi-disant rostrata des environs de Troyes, est une mauvaise copie réduite de la figure 737 de l'Iconographie de Rossmässler, figure qui doit être rapportée à la diminuata (meliùs diminuta) de Clessin. Dans cetle histoire des mollusques de France, véritable honte malacolo. gique, tout y est faux et mal nommé. C'est le plus funeste ouvrage qui ait été publiẻ sur la faune de notre pays.

Etc., etc.

La rostrata a été très bien représentée (fig. 284) par Rossmässler. Cette espèce provient du Wörthsee près de Klagenfurth, en Carniole. Je l'ai reçue également de Carinthie des viviers du docteur Ressmann, à Mlalborgeth, où il existe une variété acutalis à rostre plus aigu.

Cette Anodonte est encore répandue dans plusieurs provinces de l'Allemagne, même en Suisse où elle vit dans le lac des Quatre-Cuntons, ainsi qu'en Angleterre où elle a été recueillie à Loch-Kettrine dans le Perthshire.

Je ne la connais pas en France.

\section{Anodonta diminuta.}

Anodonta mutabilis, var. diminu a ta, Clessin Anod. $2^{\circ}$ éd. Chemnitz), p, 236, pl. 87, f. 1, 1876.

Cette Anodonte, trouvée dans l'étang des Tilleuls (Lindenweiher) près d'Interessendorf, dans le Wurtemberg, ainsi qu'a Malborgeth, en Garinthie, est bien rendue dans le travail de Clessin. 
Il faut rapporter à cet acéphale, la figure $737^{4}$ de l'iconographie de Rossmässler ainsi que la figure 5 de la planche XLVI de Moquin-Tandon (Moll. France, 1835), qui n'est qu'une reproduction réduite de celle de Rossmässler, que cet auteur a donné, de mauvaise foi, comme le portrait de la rostrata de Saint-Julien, espèce spéciale et toute différente.

La diminuta est une forme excessivement allongée, terminée par un large rostre troncatulé. La partie postérieure, comme chez les Ressmanni et Nansoutyana du groupe des Cygnnœana, égale ou dépasse même quatre fois la longueur de l'antérieur. Les sommets sont écrasés; le bord inférieur présente ordinairement un léger renflement convexe, vers sa parlie postérieure, un peu en arrière du niveau d'une verticale tombant de l'angle postéro-dorsal, ce qui donne à cette espèce une légère apparence en forme de fer de lance. Le rostre est quelquefois recourbé à son extrémité inférieure.

\section{Anodonta capitata,}

Anodonta rostrata, var. major, Brusina, Moll. Dalm. p. 131, 1866.

Anodonta capitata, Küster, in : Glessin. Anod. $\left(2^{e}\right.$ édit. Chemnitz), p. 125, pl. XXXIX, f. 1 (seulement 2), 1876.

Cette très belle espèce, fort bien figurée dans l'ouvrage de Clessin, est abondante dans la Narenta, en Dalmatie.

1. D'après un échantillon provenant du Banat.

2. La figure 2 représente incontestablement une autre forme, qui m'est inconnue. 


\section{Anodonta Sedakowi.}

Anodonta Sedakowi, Siemaschko, Bemerk land und sussw. moll. Russl. - in : Bull. acad. imp. sc. St-Petersb. VII, no 159 (sept. 1848), p. 236.

Ce mollusque, dédié à M. Sedakow par Julian-Marc de Siémaschko, vit dans le lac des 0ies (Gussinoje osero) près Werkhnendinsk, en Russie.

La Sedakowi (long. 70 ; haut. max. au niveau de l'angle postéro-dorsal, 50 ; épaiss. 28 mill.) est une forme ovale, aplatie, bien qu'un peu ventrue; sa région antérieure (long. 20 mill) est arrondie; sa région postérieure (long. 70 mill.) se termine en un rostre allongé et comprimé; les sommets rapprochés du bord antérieur sont aplatis et rugueux; la crête dorsale comprimée; le ligament postérieur médiocrement saillant; le bord supérieur rectiligne un tant soit peu convexe; l'inférieur descendant. D'après Siémascko les bords (marg.superiore ascendente, inferiore horizontali) seraient l'un ascendant, l'autre horizontal. Ces différences d'appréciation entre l'auteur russe et moi, différences qui ne sont qu'apparentes, proviennent de ce que Siémascko fait prédominer l'extrémité de la crête dorsale, tandis que moi, je donne, au contraire, aux sommets la prééminence sur toute la partie supérieure.

Quant à l'Anodonta Middendorffi, décrite en 1848 par le même auteur (p. 237) à la suite de la Sedakowi, c'est une Pseudanodonte voisine comme forme de l'elongata de Hollande. Ce qui le prouve, c'est que Siémascko, auquel un nommé Furet, de Dieppe, avait envoyé vraisemblablement des elongata, a assigné pour patrie à sa Middendorffi la 
Russie (Orel) et la France. J'ai passé intentionnellement sous silence, au commencement de ce volume, dans ma notice monographique des Pseudanodontes, cette espèce russe, parce qu'elle m'est inconnue.

\section{Anodonta sebinensis.}

Anodonta Sebinensis, Adami, mss. 1878.

Cette belle Anodonte, qui vit dans le lac d'Iseo en Lombardie, a été représentée par Clessin (Anod. pl. LV, f. 1-2) et, dans les suites à Rossmässler (fig. 1150) sous le nom erroné d'Idrina de Spinelli. Ces figures sont exactes et suffisent amplement à la connaissance de la Sebinensis.

\section{Arotonta limpida.}

Anodonta limpida, Parreyss, mss. in : Brusina, Moll.Dalm.,

p. 131, 1866, et Clessin Anod. (2ْ édit. Chemnitz), p. 126, pl. XL, f. 1-2, 1876.)

Anodonta dalmatina, Kutschig, mss. (teste Clessin, 1876.)

Acéphale très commun dans les lacs et les flaques d'eau de la partie basse de la vallée de la Narenta, ainsi que dans le lac d'Imoski, en Dalmatie.

Je l'ai reçue de Biagio Klecak sous le nom de piscinalis, var. limpida.

Elle est bien représentée sur la planche XL (fig. 1-2) de l'ouvrage de Clessin. 


\section{Anodonta Blanel.}

Gette espèce, à laquelle j'attribue le nom de M. Hippolyte Blanc, de Portici, est une Anodonte très allongée, en forme de fer de lance, dont l'arête dorsale légèrement concave et remontante à son extrémité, donne à la partie postéro-rostrale une direction sensiblement ascendante. Les valves sont fortement bâillantes, en avant et en arrière.

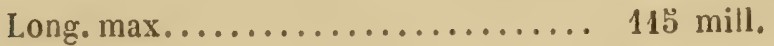

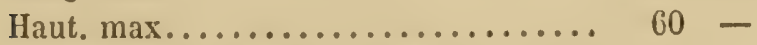

Épaiss. max. (à 29 des sommets, à 70 du rostre, à 45 du bord antér., à 35 de l'angle postéro-dorsal, à 30 de la base de la perpend.)................

Long. de la crête ligam.-dors. des sommets à l'angle postéro-dorsal...... 47 -

Dist. de cet angle au rostre.......... 53 -

Corde apico-rostrale............. 96 -

Haut. de la perpendiculaire......... 50 -

Dist. de la perpend. au bord antér.... 28 -

- du même point de cette perpend. au rostre................ 88 -

- enfin, de la base de la perpend. à l'angle postéro-dorsal....... 63 -

Bord supérieur légèrement convexe jusqu'à l'angle postéro-dorsal, puis neltement descendant, en prẻsentant un contour un tant soit peu concave. Région antérieure exiguë, arrondie et faiblement décurrente à sa base. Bord inférieur légèrement convexe, très descendant jusqu'à 35 mill. de la perpendiculaire où il forme une partie anguleuse, 
puis remontant d'une façon rectiligne, enfin, s'arrondissant en arrivant au rostre. Région postérieure très allongée (plus de trois fois plus longue que l'antérieure), terminée par un rostre arrondi, assez aigu.

Sommets écrasés, à peine proéminents (crochets excoriés), sillonnés par de fortes rides tremblotées. Arête dorsale bombée, surtout sur la région ventrale, offrant une direction légèrement concave, et allant en s'atténuant vers la partic rostrale légèrement remontante; enfin, pourvue de deux sillons presque effacés, néar,moins perceptibles, qui, des sommets, divergent jusqu'au rostre. Crête dorsale peu développée, assez comprimée.

Stries délicates sur la région ventrale, devenant ensuite grossières, très feuilletées vers les contours. Épiderme d'une teinte uniforme, se fonçant vers la périphérie et s'éclaircissant vers les sommets. Intérieur d'une nacre irisée bleuacée et orangée sous les sommets.

Ligament antéro-interne lamelleux, épais, absorbant toute la cardinale et se poursuivant à 150 mill, en arrière des crochets. Ijigament postérieur épais, assez saillant, aux trois quarts symphynoté. Lunule très allongée (long. 11 mill.). Charnière légèrement ondulée, très étroite, presque filiforme.

Cette Anodonte, que j'ai reçue sous le nom de piscinalis var. rostrata, habite l'étang de Saint-Paul, près de Thonon, en Savoie.

\section{Anodonta helvetica.}

Anodonta helvetica, Bourguignat, Malac. Quatre-Cantons, p. 59, pl. III, f. 1-2, 1862.

La figure que j'ai donnée sufnt ì la connaissance de 
cette espèce, remarquable par son arête dorsale légèrement concave, et par sa partie rostrale troncatulée, sensiblement remontante. L'helvetica est abondante dans le petit lac de Roth-Sée, près de Lucerne, en Suisse.

Mörch (Syn. Moll. Daniæ. p. 87, 1864) a signalé en Danemark, sous l'appellation d'Helvetica, une Anodonte qui ne parait pas posséder les caractères de celle de Suisse.

Cette espèce, à laquelle Mürch donne pour synonymie l'Anod. spuria d'Yoldi, mss., est une forme intermédiaire (d'après l'avis de Mörch) entre la ponderosa (fig. 282) et la rostrata (fig. 284) de Rossmässler. En tous cas, les signes distinctifs de cet Acéphale danois ne conviennent pas à mon helvetica. Ainsi, ce mollusque est : " subovalis, ventricosa, solida, postice late rostrata; area compressa, utrinque canaliculata; margo ligamentalis adscendens; rnargo postligamentalis leviter concavus. " Ce dernier caractère seul convient à l'Helvetica de Suisse; les autres ne peuvent lui être appliqués.

\section{Anodonta lacustrina,}

Anodonta mutabilis, var. 5, lacustrina. Clessin, Deutsch. Exc. Moll. fauna (4e fasc., 1877), p. 444, fig. 291.

Cet Acéphale, recueilli dans les grands lacs de la HauteBavière, est une petite forme écourtée (long. 78 mill.) très haute (haut. 51 mill.), terminée par une partie postérieure, présentant une arête concave légèrement ascendante et un large rostre obtus subtroncatulé. Ce qui caractérise surlout cette Anodonte est sa grande hauteur comparée à sa longueur. Il n'y a entre les deux mensurations qu'une différence de 27 mill. 
Clessin (loc. sup. cit.), d’après un système particulier, considère comme appartenant à deux espèces, l'une qu'il nomme mutablis, l'autre complanata, toutes les Anodontes allemandes. Je ne dirai rien de sa complanata, qui n'est pas la complanata type (fig. 6S) de Rossmässler, mais la Pseudanodonte que j'ai décrite, p. 38, sous l'appellation de Rossmässleri (fig. 283 de l'Iconographie). Je tiens seulement à exprimer ma pensée sur les figures des cinq variétés de sa mutabilis. Ainsi :

Sa mutabilis, var. $1^{\circ}$ cygncea (fig. 287) est l'Anod. pammegala, réduite de moitié.

Sa var. $2^{\circ}$ cellensis (fig. 288) me parait une cariosa à partic postérieure terminée par un rostre insolite.

Sa var. $3^{\circ}$ piscinalis (fig. 289) me scmble une furme bien voisine de l'opalina de Küster.

Sa var. $4^{\circ}$ anatina, telle qu'elle est représentée (tìg. 290), est le portrait de la Mörchiana du même auteur.

Enfin, sa var. $5^{\circ}$ lacustrina (fig. 291) est, à mon sens, une forme nouvelle el spéciale.

\section{JOURDREUILIANA}

Les Jourdheuiliana, au contraire des Rostratiana, sont des formes ovalaires, non oblongues-allongées, à convexité peu accentuée, bien régulière; convexité présentant une courbe, qui, des sommets au bord palléal, ou du bord antérieur au postérieur, diminue normalement sous la forme d'un arc parfait, bien qu'écrasé. La région postérieure se termine parfois par un rostre exigu, peu allongé, ordinairement subaigu; l'arête dorsale, par suite de la grande régularité de la convexilé, est nulle. Elle n'est 
guère perceptible que par des radiations, lorsqu'elles existent. Les sommets sont écrasés, non saillants; l'épiderme brillant, d'un assez beau coloris; les striations, enfin, sont fines et peu grossières.

Les Anodontes de ce groupe vivent dans les canaux ou les rivières vaseuses, aux eaux limpides, peu courantes.

\section{Anodonta \&ourdheuili.}

Anodonta Jourdheuili, Ray, mss.

C'est cette espèce des canaux du château des Coursà SaintJulien, à quelques kilomètres en amont de Troyes (Aube), qui a été décrite et figurée par le Sr Drouët (Naïad. France, art. 2, p. 14, pl. V, f. 2) comme le type de la rostrata, et cela, parce que cet auteur a rencontré un ou deux échantillons, où le rostre était assez prononcé. La fig. 2 (pl. V des Naïades de France) est fort bien f.ite; elle rend exactement la forme et les caractères de cette espèce, qui, chez le plus grand nombre d'échantillons, a un bec un peu moins prononcé.

Cette Anodonte, dédiée à M. Camille Jourdheuil, de Troyes, entomologiste distingué, a encore été recueillie, sans compter le châleau des Cours, dans l'Erdre, près de Nantes (Mabille).

\section{Anodonta Scaldiana,}

Anodonta Scaldiana, Dupuy, Moll. France (6॰ fasc.,185̃2). p. 613, pl. XIX, f. 12.

Cet Acéphale qu'Hécart (coq. Valenc., p. 6, 1833), avait 
prise pour l'anatina, a été avec raison distinguée par l'abbé Dupuy. Elle vit dans l'kscaut à Valenciennes.

On remarque dans les suites à Rossmässler, une Scaldiana (fig. 1960) qui n'est pas du tout la vraie Scaldiana représentée (pl. XIX, f. 12) dans l'ouvrage de l'abbé Dupuy.

L'auteur de ces suites affirme qu'il tient son espèce de l'abbé Dupuy. Eh bien! qu'est-ç que cela prouve? L'abbé Dupuy a bien envoyé, comme sa subponderosa, l'espèce nouvelle que Fagot, avec raison, a rapproché de la Castroi d'Espagne, sous l'appellation de Castropsis. J'ai bien reçu autrefois de Rossmässler, qui avait pourtant assez de coup d'œil, quelques-unes de ses espèces, qui étaient toute autre chose que ce qu'il avait publié. Cela prouve qu'il ne faut jamais accepter une espèce, même une espèce créée par un auteur, que sous bénéfice d'inventaire et sans l'avoir confrontée avec la description et la figure (type) originale.

Si l'auteur de ces suites avait agi ainsi, il aurait vu que l'échantillon que l'abbé Dupuy lui envoyait, sous le nom de Scaldiana, était une forme très différente de celle qui est représentée dans l'histoire des Mollusques de France.

Chez la scaldiana vraie la crête dorsale, nulle, est à peine comprimée; l'arête dorsale presque confondue dans la convexité régulière des valves, offre, néanmoins, deux pseudoéminences qui divergent vers le rostre; le bord inférieur, à son extrémité, n'est pas convexe-arrondi, mais rectiligne; les sommets, à peine proéminents, sont écrasés; la région antérieure est largement arrondie et non décurrente à la base; la région postérieure allongée, sans augmenter en hauteur au niveau de l'extrémité du ligament postérieur, se prolonge en s'atténuant insensiblement jusqu'à un rostre obtus, subarrondi et un peu subtroncatulé. 
Chez la Scaldiana des suites à Rossmässler, malgré que la description ait été exactement copiée dans l'ouvrage de l'abbé Dupuy, l'espèce représentée (fig. 1960) est une forme écourtée, pourvue d'une crête dorsale comprimée, élevée, à angle postéro-dorsal saillant et nettement coupé (cet angle est nul chez la vraie Scaldiana); l'arête dorsale est accentuée; les sommets sont proéminents; la région antérieure exiguë et fortement décurrente à la base; la région postérieure un peu allongée, augmente notablement en hauteur, par suite de la dilatation de la crête dorsale; le rostre est subaigu-arrondi, non largement obtus-subtroncatulé; le bord inférieur, au lieu d'être rectiligne, vers son extrémité, offre une courbe arrondie remontante vers le rostre, etc., tout, enfin, est dissemblable entre cette Scaldiana des suites à Rossmässler et la vraie de l'ouvrage de Dupuy.

$\mathrm{Si}$, enfin, l'on prend la mensuration exacte de ces deux figures, c'est-à-dire si l'on prend la mensuration en donnant aux sommets, comme de juste, la prédominance sur toute la partie supérieure, on obtient les chiffres suivants :

$$
\begin{array}{cc}
\text { Scaldiana } & \text { Scaldiana } \\
\text { de } & \text { des suites à } \\
\text { Dupuy. } & \text { Rossmässler. }
\end{array}
$$

Long. $\max . . . . . . . . . .96$ mill. $\quad 70$ mill.

Haut. max............ $52-45-$

Long. de la crête ligam.-dorsale des sommets à l'angle postéro-dorsal........ 27 - $25-$

Dist. de cet angle au rostre. $\quad 49-35$ -

Corde apico-rostrale...... $71-55-$

Haut. de la perpendiculaire. 51 - 42 - 
Dist. de la perpendicul. au bord antér........... 28 mill. 25 mill.

- du même point de cette perpend. au rostre...

- enfin, de la base de la perpend.à l'angle postérodorsal......... 55 - $47-$

Sans tenir comple des différences de taille, trois mesures frappent par leurs discordances.

$1^{\circ}$ La région postérieure de la vraie Scaldiana est plus de deux fois plus longue (61 mill.) que l'antérieure, qui n'a que 28 mill. Celle de la fausse Scaldiana n'atteint pas deux fois ( 45 mill.) celle de l'antéricure (25̆ mill.). Il résulte de là que la vraie (proportion gardée) a une région posté. ricure plus allongée que celle de la fausse scaldiana.

$2^{\circ}$ La crête dorsale, des sommets à l'angle postéro-dorsal, est presque aussi longue chez l'une (27 mill.) que chez l'autre (25 mill.). Mais, où les différences s'accentuent, c'est de l'angle postéro-dorsal au rostre. Chez la vraie Scaldiana, la distance atteint 49 mill.; chez la fausse, 35 mill. seulement.

$3^{\circ}$ Chez la scaldiana type, il y a une différence de 1 mill. entre la hauteur maximum et celle de la perpendiculaire; de plus, ces deux lignes de hauteur sont à peine distantes l'une de l'autre. Chez la fausse de l'auteur allemand, la hauteur maximum ( $40 \mathrm{~b}$ mill.) se trouve en arrière de 20 mill. de la perpendiculaire, qui ne dépasse pas 42 mill., il résulte de là que, chez la vraie, la région postérieure va toujours en diminuant de hauteur, à partir de la perpendiculaire, tandis que chez la fausse, celte région va en augmentant presque jusqu'à l'extrémité de 
la crête dorsale, pour s'atténuer ensuite subitement.

11 ressort de ces chiffres que ces deux Scaldiana, très différentes sous tous les rapports, ne peuvent être considérées comme une même espèce. La Scaldiana de l'auteur allemand, en effet, bien qu'elle ait été envoyée par l'abbé Dupuy, sous cette dénomination, est tout simplement la subarealis de notre ami P. Fagot de Villefranche, Anodonte abondante dans les eaux de France, du Nord au Midi.

\section{Anodonta Serbicn.}

Anodonta Serbica, Letourneux, in litt.

Espèce de forme ovalaire, à extrémité atténuée en un rostre assez aigu. Valves peu ventrues, paraissant même assez comprimées, relativement épaisses, bâillantes seulement en arrière entre l'angle postéro-dorsal et le rostre. Convexité peu accentuée, néanmoins bien régulière.

Long. $\max . \ldots \ldots \ldots \ldots \ldots \ldots \ldots . . . .61$ mill.

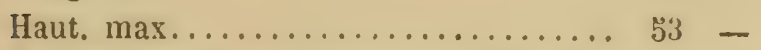

Épaiss. max. (à 28 des sommets, à 48 du rostre, à $38 \mathrm{du}$ bord antér., à 26 de l'angle post.-rlors., à 28 de la base de la perpendiculaire).............. 26 -

Long. de la crête ligam.-dors., des sommets à l'angle postéro-dorsal........... 31 -

Dist. de cet angle au rostre.......... 48 -

Corde apico-rostrale.............. 73 -

Haut. de la perpendiculaire......... 48 Mar. 1881 . 
Dist. de cette perpend. au bord antérieur. 23 mill.

- du même point de cette perpend. au rostre.................. $6 \ddot{8}$ -

- enfin, de la base de la perpend. à l'angle postéro-dorsal............ 53 -,

Bord supérieur rectiligne jusqu'à l'angle postéro-dorsal, puis descendant brusquement sur le rostre en oflrant un contour un tant soit peu concave. Région antérieure arrondie, légèrement décurrente à la base. Bord inférieur régulièrement arqué-convexe jusqu'au rostre, dans une direction un peu descendante. Région postérieure près de trois fois plus longue que l'antérieure, terminée par une partie rostrale inférieure, arrondie et assez aiguë.

Sommets écrasés, non proéminents (crochets aigus), sillonnés de rides délicates tremblotées. Arête dorsale confondue dans la convexité des valves, s'accusant seulement par la présence de deux radiations vertes divergentes. Crête dorsale peu développée, faiblement comprimée vers l'angle postéro-dorsal.

Stries fines, régulières, seulement plus forles et même feuilletées sur les contour's. Épiderme brillant, d'une belle teinte jaunacée-verdâtre, s'éclaircissant sur la région ventrale, passant parfois, comme chez les échantillons de Krapina, à une nuance rougeâtre vers les sommets, enfin, présentant de nombreuses radiations vertes seulement vers les contours et notamment sur la région postérieure. Intérieur d'une belle nacre bien irisée, d'une nuance bleuacie à reflets violacés.

Ligament antéro-interne fort, épais, s'étendant à 150 millimètres aussi bien en avant qu'en arrière des crochets. 
Ligament postérieur médiocre, presque entièrement symphynoté. Lunule grande, allongée.

La Serbica a été recueillie par notre ami le conseiller Letourneux, dans le Danube, aux environs de Belgrade (Serbie), ainsi que dans la rivière de Krapina-Tœplitz, en Croatie.

\section{Anodonta horolsi.}

Petile espèce très brillante, bien ovale, à convexité médiocre, parfaitement régulière. Valves assez épaisses, offrant deux bâillements : un fort petit, réduit à l'état de fente, à la partie inféro-antérieure, et, un autre très ouvert, du rostre à l'angle postéro-dorsal.

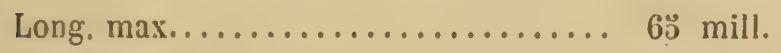

Haut $\max . . . \ldots \ldots \ldots \ldots \ldots \ldots . . .639-$

Épaiss. max. (à 16 des sommets, à 39 du rostre, à 27 du bord ant., à 19 de l'angle postéro-dorsal, à 23 de la base de la perpendiculaire).............. 19 -

Long. de la crête ligam.-dors., des sommets à l'angle postéro-dorsal....... 26 -

Dist. de cet angle au rostre........... 31 -

Corde apico-rostrale............. 54 -

Haut. de la perpendiculaire.......... 36 -

Dist. de cette perpend. au bord antér.... 19 -

- du même point de cette perp. au rostre. $45-$

- enfin, de la base de la perpend. à l'angle postéro dorsal.......... 39 -

Bord supérieur convexe jusqu'à l'angle postéro-dorsal, qui est fort obus et presque nul, puis descendant d'une 
façon rectiligne sur le rostre. Région antérieure bien ronde. Bord inférieur régulièrement convexe jusqu'au rostre. Région postérieure allongée, plus de deux fois plus longue que l'antérieur, s'atténuant en une partie rostrale obtuse-arrondie.

Sommets (toujours excoriés) aplatis, non saillants. Arête dorsale nulle. Crête dorsale à peine développée et faible . ment comprimée.

Stries prononcées, régulières, plus fortes et feuilletées vers les contours. Epiderme brillant, d'un jaune-noirâtre passant au vert à la partie supéro-postérieure. Intérieur d'une nacre bleuacée, plombée sous les sommets.

Ligament antéro-interne épais, absorbant toute la caldinale. Ligament postérieur médiocre, presque entièrement recouvert. Lunule grande, triangulaire. On remarque à l'extrémité du ligament interne, au commencement de la partie latérale de la charnière, une éminence tuberculeuse assez forte.

Cette Anodonte, que je dédie à notre regretté ami, Lorois de Broël, malacologiste et ancien préfet du Morbihan, vit dans le canal de Bretagne, à Saint-Congard (Morbihan). Le docteur Rambur a découvert cette espèce aux environs de Tours.

\section{H g ANATHNANA}

Les espèces de ce groupe sont remarquables par leurs valves oblongues, plus ou moins allongées, en forme de fer de lance. La région antérieure, toujours fort exiguë, est décurrente à la base. Les sommets très oblus, non proéminents, sont très rapprochés du bord antérieur. La région 
postérieure, généralement deux ou trois fois plus longue que l'antérieure, se développe en hauteur, ordinairement jusqua'au niveau d'une verticale tombant de l'extrémité du ligament postérieur. La partie rostrale est arrondie-obtuse, ou bien troncatulée.

Les Anatiniana vivent indifféremment dans les eaux paisibles ou courantes, on les trouve aussi bien dans les lacs, les canaux, que dans les ruisseaux et les rivières.

\section{Anodonta Krapinensis.}

Anodonta Krapinensis, Letourneux, mss.

Belle espèce oblongue, à rostre tronqué et à valves d'un beau coloris, bâillantes en arrièr et en avant.

Long. $\max . . . . . . . . . . . . . . . .93$ mill.

Haut. $\max . . . \ldots \ldots \ldots \ldots \ldots . . . .63-$

Épaiss. max. (à 28 des sommets, à 50 du rostre, à $43 \mathrm{du}$ bord antér., à 27 de l'angle postéro-dorsal, à 29 de la base de la perpendiculaire)........... $2 T$ -

Long. de la crête ligam.-dors., des sommets à l'angle postéro-dorsal.......: : 7 -

Dist. de cet angle au rostre.......... 43 -

Gorde apico-rostrale.............. 7 วั -

Haut. de la perpendiculaire......... 48 -

Dist. de cetle perpend. au bord antér.... 26 -

- du même poinl de cette perpend. au rostre.................. 68 -

- enfin, de la base de la perpend. à l'angle postéro-dorsal......... รั4 - 
On remarquera que l'épaisseur maximum est presque centrale, à quelques millimètres près. La convexité est régulière de tous côtés, sauf entre l'arête et la crête dorsale où elle descend assez brusquement. La plus grande hauteur (๖3 mill.) est à 26 mill. en arrière de la perpendiculaire.

Bord supérieur légèrement arqué jusqu'à l'angle postéro-dorsal, puis descendant en ligne droite de cet angle au rostre. Région antérieure arrondie, décurrente à la base. Bord inférieur convexe-descendant jusqu'au point de sa plus grande hauteur, puis légèrement remontant. Région postérieure allongće, dans une direction faiblement descendante, deux fois et demie plus longue que l'antérieure, augmentant sensiblement jusqu’à 26 mill. en arrière de la perpendiculaire, ce qui lui donne une apparence de fer de lance, enfin, se terminant par un rostre large et nettement troncatulé.

Sommets peu ventrus, non proéminents, comme obtus. écrasés, et sillonnés par de fines stries émoussées. Crochets aigus. Arête dorsale prononcée surtout vers la région ombonale, offrant deux sillons obsolètes, divergents et allant aboutir aux extrémités (supérieure et inférieure) du rostre. Crête dorsale assez développée et notablement comprimée, surtout vers l'angle postéro-dorsal.

Stries régulières, assez délicates sur la partie ventrale, devenant plus fortes vers les contours et feuilletées notamment à la partie postérieure. Épiderme brillant, d'un beau vert-jaunacé, passant au vert-noir de l'arête à la crête dorsale, ct au rouge brique vers les sommets. Inté. rieur d'une nacre irisée d'un blanc bleuacé.

Ligament antéro-interne épais, plus porté en avant qu’on arrière des crochets. Ligament postérieur allongé, 
peu saillant, presque entièrement recouvert. Lunule très allongèe (9 mill.)

Cette Anodonte vit dans la rivière de Krapina, en Croatie, où elle a été recueillie par notre ami le conseiller Letourneux.

\section{Anodionta Helnmopsis,}

Long. $\max . \ldots \ldots \ldots \ldots \ldots \ldots \ldots . . . . . . .68$ mill.

Haut. $\max . . . \ldots \ldots \ldots \ldots \ldots . . . . .51-$

Épaiss. max. (à 26 des sommels, à $56 \mathrm{du}$ rostre, à 42 du bord ant., à 26 de l'angle postéro-dorsal, à 29 de la base de la perpendiculaire)................. 30 -

Long. de la crête ligamento.dorsale, des sommets à l'angle postéro-dorsal.... 38 -

Dist. de cet angle au rostre......... 51 -

Corde apico-rostrale............. 81 -

Haut de la perpendiculaire.......... 43 -

Dist. de cette perpend. au bord antér... 24 --

- du même point de cette perp. au rostre.................... 74 -

- enfin, de la base de cette perpend. à l'angle postéro-dorsal......... fir. -

Cielte espèce, plus allongée et moins haute que la pré. cédente, puisqu'elle a $98 \mathrm{mill}$. de long, sur $51 \mathrm{mill}$. de hauteur, offre également une épaisseur maximum presquıe médiane entre les sommets, l'angle postéro-dorsal et la base de la perpendiculaire; seulement, chez celle-ci, cette épaisseur, par suite du prolongement plus grand de la région postérieure, se trouve plus rapprochée du bord an- 
lérieur. La convexité, chez l'cechmopsis, n’est pas régulière. Très accentuée sur la région de l'arête dorsale, elle descend vers le bord palléal d'une manière déclive, non bombée. Elle offre, de plus, sur sa région postérieure plusieurs zones divergentes de bombements inérgaux; enfin, entre I'arête et la crête dorsale, elle s'atténue plus brusquement que celle de la Krapinensis.

Comme aspect l'xchmopsis a tout à fait une apparence très allongée en forme de fer de lance. Son borả supérieur rectiligne, et, son inférieur, fort peu convexe, donnent à cette Anodonte un cachet différent de celui de la Krapinensis.

Chez l'xchmopsis, la ligne de la plus grande hauteur, à 31 mill. en arrière de la perpendiculaire, coïncide avec une verticale qui tomberait de l'extrémité du ligament postérieur. Chez la Krapinensis, cette ligne n'est qu’à 26 mill. De plus, chez l'xchmopsis, il y a une diffírence de 8 mill. entre la hauteur de la perpendiculaire et celle de la hauteur maximum, tandis qu'elle n'est que de 5 mill., entre les deux hauteurs, chez la Krapinensis. Il résulte de là que l'æchmopsis, bien qu'elle soit plus allongée, est moins haute que la Krapinensis.

Cơ. à valves assez minces, offrant deux bâillements, un en avant et un autre en arrière, entre le rostre et l'angle postéro-dorsal.

Bord superieur rectiligne jusqu'à l'extrémité du ligament, puis descendant sur le rostre en présentant un contour légèrement concave. Région antérieure peu léve loppée, arrondie, décurrunte à la base. Bord inférieur faiblement convexe-descendant jusqu'à 31 mill. en arrière de la perpendiculaire, puis remontant d'une façon rectiligne vers le rostre. Région pustérieure très allongée, plus 
de trois fois plus étendue que l'antérieure, allant en augmentant jusqu'au niveau d'une verticale tombant de l'extrémité du ligament postérieur, puis s'atténuant, pour se terminer par un large rostre troncatulé.

Sommets très obtus, assez ventrus, bien que peu proéminents, enfin, sillonnés par de petites rides tremblotées. Crochets aigus, écrasés. Arête dorsale très bombée vers la région ombonale, allant ensuite en s'atténuant régulièrement jusqu'au rostre, en présentant deux sillons très émoussés, divergents et se terminant aux extrémités (supérieure et inférieure) de la partie rostrale. Crête dorsale non saillante, mais bien comprimée.

Stries plus ou moins saillantes, suivant les échantillnns, généralement fines sur la région ventrale, et prononcées, même un peu feuilletíes, vers les contours. Épiderme brillant, d'une teinte cendrée-jaunacée ou fauve-rougeâtre, passant an ronge-brique vers les sommets et au rouge-noinäire sur la ré́gion postéro dorsale. Intérieur d'une nacre bleuacée-blanchâtre peu irisée, prenant un ton orangé sous les sommets.

Ligament antéro interue sans particularité. Ligament postérieur médiocre, allongé, presque entièrement recouvert. Lunule grande, subtriangulaire.

L'æchmopsis vit dans le lac du Bourget, du côté de Tresserve, en Savoie. Elle habite également aux environs de Krapina, en Croatie, où elle a été recueillie par le conseiller Letourneux.

Anodorata Filariana.

Long. $\max . . . \ldots \ldots \ldots \ldots \ldots . . . . .65$ mill.

Haut. $\max \ldots \ldots \ldots \ldots \ldots \ldots \ldots$ \& 4. 
Épais. max. (à 24 des sommets, à 50 du rostre, à 34 du bord antér., à 27 de l'angle postéro-dorsal, à 22 de la base de la perpeid..................

Long. de la crête ligramento dorsale des sommets à l'angle postéro-dorsal... 34 -

Distance de cet angle au rostre....... $42 \quad-$

Corde apico-rostrale............ 70 -

Hauteur de la perpendiculaire....... 43 -

Dist. de la perpend. au bord antér..... 24 -

- du même point de cette perpend. au rostre....................

- enfin de la base de la perpend. à l'angle postéro-dorsal............

Gette Anodonte, moins en forme de fer de lance que la précédente, ost oblongue dans une direction descenlante. Les valves offrent deux médiocres bâillements en avant et en arrière. A l'état jeune, la Pilariana a un aspect un peu ellipsoïde, et son arête dorsale est un tant soit peu concave.

La convexité, chez cette espèce, est régulière, sauf entre l'arête et la crête dorsale, où elle descend assez brusquement, la plus grande hauteur est à 26 mill. en arrière de la perpendiculaire.

Bord supérieur très faiblement convexe-descendant jus. qu’à l'angle postéro-dorsal, qui est très émoussé et non saillant, puis, à partir de cet angle, ofirrant un contour descendant un tant soit peu concave vers sa partie stipérieure. Région antérieure bien arrondie, peu décurrente à la base. Bord inférieur convexe-descendant, puis fablement remontant à son extrémité. Rímion postérieure, lé- 
passant $\in$ n longueur plus de deux fois et demie celle de l'antérieure, s'atténuant en une partie rostrale obtusearrondie.

Sommets très obtus, émoussés, non proéminents (toujour's excoriés), sillonnés par d'assez larges rides espacées, dont on aperçoit ies traces sur la nacre sous-épidermique. Arête dorsale assez accentuée, pourvue de deux zonules foncées, divergentes, et offrant supérieurement, entre elle et la crête, une atténuation assez brusque, qui paraît légèrement concave, par suite de la compression de la crête vers l'angle postéro-dorsal.

Stries régulières, assez. fortes, devenant grossières et feuilletées vers les contours. Épiderme d'un coloris brillant, d'un marron très funcé teinté çà et là de tons jaunacés ou verdâtres, passant vers les contours à une nuance noirâtre et, vers les sommets, à une autre rougeâtre plus claire. Intérieın d'nnn nacre bleuacée bien irisée.

Ligameis ...t ci.c-........e filiforme. Ligament postérieur médiocre, presque entièrement recouvert. Lunule triangulaire, très allongée.

Cette espèce, à laquelle j'attribue le nom du docteur Pilar, professeur de géologie à l'Université d'Agram, a été trouvée dans la rivière de Krapina et dans la Save à Agram, par le conseiller Letourneux.

\section{Anodonta Rayl.}

Anodonta Rayi, Durouy, Cat. Extram. Galliæ, test. $n^{\circ} 2$. 1849 , et Hist. Moll. France ( $6^{\circ}$ fasc. 1852), p. 614 , pl. XX, f. 22.

La Rayi est bien renrésentéc dans l'ouvrage de l'abbé 
Dupuy. C'est une Anodonte en forme de fer de lance, à partie antérieure exiguë, fortement décurrente à sa base. Sa région postérieure va toujours en augmentant en hauteur jusqu'au niveau de l'extrémité du ligament postérieur.

Cette espèce a été décourerte par notre ami Ray,à Troyes dans le ruisseau de la Bonde-Gendret, ruisseau actuellement recouvert par une voûte. Elle a encore été recueillie dans différents bras de la Seine à Troyes et à Nogent-surSeine, ainsi que dans la Nosle à Aix-en-Othe (Aube).

Je l'ai trouvée dans le lac des Quatre-Cantons près de Kussnacht, en Suisse.

\section{Anocionta danica.}

Anodonto dariica, Mörch, in litt.

C'est cet Acéphale que Mlörch (Syn. moll. Daniæ, p. 89, 1864) avait autrefois regardée comme l'anatina de Linnocus. Elle paraît très répandue dans les eaux du Danemark.

La Danica, bien que Mörch l'ait qualifiće d'ovala, est une Anodonte très allongée, moins haute que la Rayi, et également en forme de fer de lance. Ses sommets sont plus antérieurs; sa convexité est peu régulière, enfin, sa région antérieure est nlus réduile et plus écourtíe. 


\section{Anodonta anatina.}

Mytilus anatinus, Linnœus, Syst. nat., p. 706, no 219 , édit. X, 1758, - et Hanley, ipsa Linṇœi conchylia, p. 144, pl. II, f. 1 (optima), 1855 .

Anodonta anatina, Rossmässler, Iconogr. V et VI, 1837, fig. 417 (seulement).

L'anatina de Linnoeus est une petite Anodonte ressemblant à la Rayi, mais à région postérieure très écourtée, termince par une partie rostrale largement obluse. La figure donnée par Hanley, d'après le type linnéen, est excellente; celle de Rossmässler vaut beaucoup moins. Ces deux figures sont suffisantes pour la connaissance de cette espèce.

L'anatina paraît peu commune. On la rencontre en Suède, en Angleterre, en Allemagne, en Suisse et en France.

Je la connais, de l'Yvette, à Chevreuse, dans Seineet-Oise (Mabille); du canal de l'Ourcq à Bondy, près Paris (Mabille); de la Masle, à Amboise (Rambur); de l'Areyron, à Rhodez (Malafosse); du Menthon, dans l'Ain (Locard); des environs de Lons-le-Saulnier et de Saint-Amour dans le Jura; enfin, du lac de Neuchâtel, en Suisse.

Tous les auteurs admettent l'anatina de Nilson (Hist. Moll. Sueciæ, p. 114, 1822) comme l'espèce type de Linnœus, ce qui est complètement erroné, l'anatina de l'auteur suédois se compose de trois formes distinctes, comme 
on peut s'en convaincre en se reportani aud synonymies données par Nilson.

Lorsı̣u'on parcourt les ouvrages, on reconnaît qu'il y a. bien peu de malacologistes qui ont connu l'espèce linnéenne, ainsi :

Le Mytilus anatinus de Schröter (flussconch., p. 160, pl. I, fig. 2 et 3,1779 ) est formé de deux Anodontes différentes: Ia première (fig. 2) de Weimar, est l'intermedia de Lamarck; la seconde (fig. 3) de la Saale en Bavière, est une petite forme voisine de la minima de Millet.

Le Mytilus anatinus de Maton et Rackett (cat. test. gr. Brit. in : trans. Linn. soc. London, VIII, 1804, - et, traduction Chenu, 1845, p. 163, pl. XVI, tig. 1, et var. fig. 4) se rapporte également à deux espèces distinctes: $1^{\circ}$ la fig. 1 (pl. XVI) à la Cygnaca; $2^{\circ}$ la fig. 4 à la fallax de Colbeau.

Le Mytilus anatinus du révérend Sheppard, publié dans le tome XIII (1820), des transactions de la Société linnéenne de Londres (traduction Chenu, p. 270, pl. XXVI, fig. 1) est mon Anodonta gallica. C'est dans ce mémoire (On two new Brit. spec. of Mytilus) que Sheppard a décrit deux espèces, dont je parlerai plus tard, les incrassatus et macula.

L'Anotonta anatina de Draparnaud (Hist. Moll., pl. XII, f. 2,1805 ), telle qu'elle est figurée, ne ressemble à rien de ce que je connais. Elle me paraît une forme peu adulle du groupe des Cygnoeana. Celte figure, du reste, est fort médiocre.

L'anatina de l'abbé Dupuy (Hist. Moll. France, $6^{\circ}$ fasc., 1852, pl. XIX, f. 13) est la subarealis de Fagot.

L'anatina de Moquin-Tandon (Moll. France, II, 1855, p. こૅ58) est un amalgame insensé de toutes sortes de for- 
mes. Quant a la forme figurée sous l'appellation d'anatina (pl. XLV, f. 1 et 2), c'est une mauvaise reproduction de la scaldiana de Dupuy.

L'anatina de Brown (illust. conch. Gr. Brit. 1845, pl. XIII) est cette magnifique Browni, que j'ai dédiée i cet auteur.

L'anatina de C. Pfeiffer (Naturg. Deutsch. Moll., 1, 182I, p. 112, pl. VI, f. 2.) est une forme voisine de l'oblonga de Millet.

L'anatina de Rossmässler (iconogr. V et VI, 1837) comprend: $1^{\circ}$ (fig. 417) l'anatina de Linnœus; $2^{\circ}$ (fig. 418) une forme du Weser, vraisemblablement nouvelle et qui m'est inconnue; enfin, $3^{\circ}$ (fig. 419 et 420 ) des variétés de la subluxatu de Küster.

L'anatina de Glessin (Anod. 20 édit. Glıemnitz, p. 83, 1870்) s'appliqque à deux espèces : $1^{\circ}$ calle de la pl. XIV, f. 3-4, du Mecklembourg, est une forme subtrigone, très bombée et bien voisine de la parvula; $2^{\circ}$ celle de la pl. XXVI, f. 2, est la copie de cette anatina du Weser, représentée (fig. 418) par Rossmäisler, et qui m’est inconnue.

Dans un autre de ses ouvrages (Deutsch. exc. Moll. fauna, $4^{0}$ fasc. 1877 , (ig. 290), Clessin a donné comme anatina, sa Morchiana.

lite..., etc...

Il y a encore beaucoup d'autres anatina ligurées par les auteurs, mais j'aime micux les passer sous silence, parce que je n'en finirais point. Quant à celles des Naïades de l'Aube et du Lémun, do MINI. Brot et Drouët, j'ai dit ce que j'en pensais au commencement de ce mémoire. 


\section{Anodonta palngtris.}

Anodonta palustris, d'Orbigny, in : Ferussac. Art. Anod., in : Dict. class. Hist. nat., I, 1832, p. 397.

Anodonta alpestris, Charpentier, mss.

Anodonta tenella, Held, in : Kuster, g. Anod., $2^{\circ}$ édit., Chemnitz, p. 63, pl. IX, f. 5, 1852.

Ferussac en citant, dans le Dictionnaire classique d'Histoire naturelle, cette espèce d'Alcide d'Orbigny, n'en a pas donné les caractères. Il a seulement dit que cette Anodonte était celle publiée et figurée sous le nom de Mytilus anatinus, par Mendez de Costa (Brit. conch., p. 215, pl. XV, fig. 2, 1778). Or, lorsqu'on se reporte à l'ouvrage de da Costa, on reconnaît que cet anatinus est semblable comme forme et comme caractire à l'alpestris de Charpentier, espèce molheureusement manuscrite, qui n'est autre chose que la tenella de Held, décrite el figurée par Küster (§. Anod., p. 63, pl. IX, f. 5, 1852).

La palustris vit en Auvergue (Alc. d'Orbigny). Elle a élé citée de cette contrée par Bouillet (Moll. Auverg., p. 80, 1836). Je la connais, en outre de Chalon-sur-Saône (Fagot), du ruisseau de la Salle, dans Saône-et-Loire (Locard), du canal entre Loir-et-Cher, près de Tours (Rambur), du Maine, à Angers (Mabille), etc.

Küster l'indique, sous le nom de tenella, des environs de Wasserl)urg, en Bavière. Je l'ai recueillie dans le lac des Quatre-Cantons, près de Kussnacht, en Suisse. Je l'avais confondue autrefois, alors que je n'étais pas aussi versé que je le suis actuellement dans l'étude des Anodontes, avec l'anatina. 


\section{LUSTTANIANA}

Les Acéphales de ce groupe, bien que voisins des précédents, sont des espèces subtrigonales, à région antérieure très réduite, comme rostrée, ct à région postérieure largement dilatée-obtuse. Celte partie postérieure est relativement si développée en hauteur et l'antérieure est si exiguë, que ces Acéphales paraissent comme coniques.

Je ne connais que trois Lusitaniana, une du Portugal, une seconde de France, enfin, une troisième de Suisse.

\section{Amodonta lusitana.}

Anodonta lusitana, Morelet, Moll. Portugal, p. 103, pl. XII, f. $1,184 \%$.

Cette espèce est parfaitement figurée dans l'ouvrage de Morelet. Cetle figure suffit amplement pour la connaissance de cette Anodonte. Cette forme habite dans les affluents de la Guadiana, affluents qui descendent des hautes vallées des Algarves, entre Mertola et Castro-Verde.

\section{Anodonta BBourguignati.}

Anodonta Bourguignati, Mabille, in litt.

Je ne puis mieux définir cette espèce qu'en disant qu'elle est une miniature de la lusitana. Ce sont les mêmes contours, la même forme, à peu près le même aspect, et pour ainsi dire une semblable coloration. Seulement, chez la MAI 1881. 
Bourguignati, les sommets sont plus antérieurs, et il existe pas, chez elle, cette légère concavité qui limite la partie supérieure du rostre de la lusitana.

Long. $\max . . . . . . . . . . . . . . . .664$ mill.

Haut. max................. 40 -

Epaiss. max. (à 24 des sommets, à 30 du rostre, à $33 \mathrm{du}$ bord antér., à $22 \mathrm{de}$ l'angle postéro-dorsal, à 18 de la base de la perpend.)................

Long. de la crête ligam.-dors., des sommets à l'angle postéro-dorsal .......

Dist. de cet angle au rostre...........

Gorde apico-rostrale...............

Haut. de la perpendiculaire..........

Dist. de la perpend. au bord antér.....

- du même point de la perpend. au

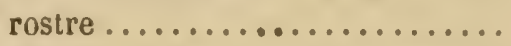

- enfin, de la base de la perpend. à l’angle postéro-dorsal.........

On remarquera : 10 que l'épaisseur maximum est plus rapprochée du rostre que du bord antérieur, et moins distante de la base de la perpendiculaire que des sommets et de l'angle postéro-dorsal; $2^{\circ}$ qu'il y a une différence de 7 mill. entre la plus grande hauteur et celle de la perpendiculaire ; $3^{\circ}$ enfin, que la hauteur maximum ( 40 mill.) est relativement énorme pour la longueur (64 mill.).

Coq. de forme trigonale, à valves minces, bâillantes: $1^{-}$dans tout le contour antérieur et palléal; $2^{\circ}$ et entre le rostre et l'angle postéro-dorsal.

Bord supérieur rectiligne, puis bien arqué-convexe, à 
partir de l'extrémité du ligament jusqu'au rostre. Région antérieure très exiguë, arrondie el décurrente à la base. Bord inférieur convexe-descendant, ensuite convexe-remontant à son extrémité. Région postérieure plus de deux fois plus longue que l'antérieure, allant en augmentant en hauteur jusqu'à 22 millimètres en arrière de la perpendiculaire, où elle atteint 40 mill., puis se terminant par une large partie rostrale arrondie, excessivement obtuse, sans rostre défini.

Sommets comprimés, comme aplatis, sillonnés de jolies rıdes rectilignes, inclinées de gauche à droite et coupant obliquement les petites lignes concentriques que l'on remarque entre l'intervalle des rides. Grochets très aigus. Arête dorsale nulle, confondue dans la convexité régulière des valves. Crête dorsale assez développée et comprimée vers l'angle postéro-dorsal, qui est très obtus et arrondi.

Stries fines, délicates, devenant grossières vers les con. tours. Épiderme presque toujours encrassé d'un limon rougeâtre, offrant, lorsqu'il est enlevé, un coloris d'un ton jaunacé vert d'eau, devenant plus clair sur la région ventrale et passant au rouge sur les sommets. Intérieur d'une nacre blanche bleuacée, bien irisée.

Ligament antéro-interue filiforme. Ligament postérieur médiocre, presque recouvert. Lunule très grande.

La Bourguignati, que notre ami Mabille a bien voulu me dédier, habite dans le canal de l'Ourcq, à Bondy, près de Paris.

\section{Anodonta ruvida.}

La ruvida diffère de la précédente par sa taille un peu moins longue et un tant soit peu plus haute; par ses som- 
mets encore plus en avant, et une régrion antérieure plus réduite, comme anguleuse. Chez la ruvida, le bord supérieur est convexe, au lieu d'être rectiligne; le contour, entre l'angle postéro-dorsal et le rostre, est légèrement concave; la partie rostrale est plus largement arrondie; enfin, la convexité maximum est peu distante des sommets et du bord antérieur. Chez la Bourguignali, à l'inverse de celle-ci, la convexité, plus centrale, est plus rapprochée du rostre et de la base de la perpendiculaire.

Long. $\max . . . \ldots \ldots \ldots \ldots \ldots . . . .61$ mill.

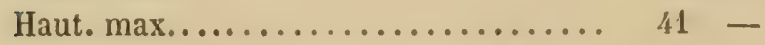

Epaiss. max. (à 15 des sommets, à 39 du rostre, à 25 du bord antérieur, à 21 de l'angle postéro-dorsal, à 24 de la base de la perpend.)................

Lung. de la crête ligam.-dors., des sommets à l'angle postéro-dorsal.......... 31 -

Dist. de cet angle au rostre.......... $32-$

Corde apico-rostrale............. 54 -

Haut. de la perpendiculaire.......... 34 -

Dist. de cette perpend, au bord antér... 16 -

- du même point de cette perpend.au rostre.................. 46 -

- enfin, de la base de la perpend.à l'angle postéro.dorsal........... 40 -

D'aprìs ces mensurations, on remarque qu'il n'y a qu'une différence de 1 mill. entre la longueur (31 mill.) de la crête dorsale et la distance ( $\$ 2$ milll.) de l'angle au rostre; tandıs que, chez la Bourguigrati, la diflérence est de 5 mill. (la crète dorsale de cette espèce n'a que 29 mill. contre 34 mili.. distance de l'angle au rostre. Il résulte de 
là que la crête de la ruvida est plus développée que celle de la Bourguignati. On remarque encore que la région postérieure a, chez ces deux espèces, une égale longueur de 46 millim., tandis que la région antérieure n'a que, 16 mill. chez la ruvida, lorsqu'elle atteint 19 mill. chez la Bourguignati.

Cioq. de forme obtusément subtrigonale, à valves mincEs, grossièrement striées, et offrant deux faibles bâillements, l'un antérieur, l'autre postérieur.

Bord supérieur arqué, ensuite légèrement concave, à partir de l'angle jusqu'au rostre. Région antéricure excessivement réduite, subarrondie avec une légère partie anguleuse vers le milieu de son contour, enfin, décurrente à sa base. Bord inférieur fortement descendant presque en ligne droite, puis s'arrondissant à 11 mill. de la perpendiculaire, pour remonter vers le rostre. Région postérieure près de trois fois plus longue que l’antérieure, très dilatée el largement termínée par une partie rostrale, bien arrondie, regardant en bas.

Sommets très antérieurs, un peu convexes, faiblement ventrus, non aplatis, sillonnés par de très fines petites rides concentriques. Grochets aigus. Arête dorsale confondue dans la convexité, qui n'est pas aussi régulière que la prícédente. Grète dorsale plus dilatée et très comprimée vers l'angle, qui est fort émoussé.

Stries grossières, comme rugueuses et feuilletées. Épiderme d'une nuance presque uniforme, jaunacée-verdâtre ou ocracće-brunâtre, s'éclaircissant vers les sommets. Intérieur d'une nacre blanche bien irisée.

Ligaments: antéro-interne mince, très antérieur; postérieur mćdiocre, assez recouvert. Lunule très allongée (9 mill.). 
La ruvida vit dans le lac de Zurich, près la ville du même nom, aiısi que dans le lac de Neuchâtel, en Suisse, où elle a été recueillie par M. G. Coutagne.

\section{I ๑० WESTERLUNDYANA}

Les Westerlundiana ont souvent été prises pour des anatina. Ce sont des espèces plus ou moins oblongues, non en forme de fer de lance; à région antérieure développée dans le sens de la hauteur; à crête dorsale atténuée, souvent presque nulle; à convexité règulière; enfin, à région postérieure allongée dans une direction descendante et terminée par une partie rostrale obtuse.

Ces Anodontes habitent indifféremment les lacs et les rivières.

\section{Anodonta glabra.}

Anodonta glabra 1, Villa, Disp. syst. conch., p. 40 (sans caractères), 1841.

Cette espèce signalée de Carinthie par Villa, m’a élé envoyée, sans indication de localités, par ce même auteur, il y a une vingtaine d'années, comme provenant de Lombardie. Je ne sais, en léfinitive, si cette Anodonte est commune à ces deux pays, ou, si elle est particulière à la Carinthie, ou spéciale à la lombardie. En tous cas, elle habite en France, d'où je la connais, de la Couze à Issoire, dans le Puy-de-Dôme, et de la Loire à Roanne (Fagot).

1. Il existe une Anodonta glabra de Ziégler, mss qui m'est inconnue. 
Les échantillons de ces localités sont identiques à l'individu que je tiens de la libéralité de Villa, individu qui devient le type de cette nouvelle Anodonte, puisque cette espèce n’a jamais été décrite.

Long. $\max . \ldots \ldots \ldots \ldots \ldots \ldots . . . . .65$ mill.

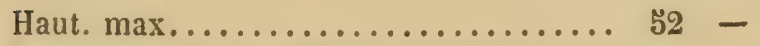

Épaiss. max. (à 24 des sommets, à 49 du rostre, à 38 du bord antér., à 21 1/2 de l'angle postéro-dorsal, à 31 de la base de la perpend.)................

Long. de la crête ligamento-dorsale, des sommets à l'angle postéro-dorsal..... 34 -

Dist. de cet angle au rostre........... 45 -

Corde apico-rostrale............. 72 -

Haut. de la perpendiculaire.......... 48 -

Dist. de cette perpend. au bord antérieur. 25 -

- du même point de cette perpend. au rostre................... 60 -

— enfin,de la base de la perpend. à l'angle postéro.dorsal.............. 引1 -

Coq. de forme oblongue dans une direction légèrement descendante, à valves assez minces, très brillantes, d'un beau coloris, à peine bâillantes, en avant et cu arrière.

Bord supérieur faiblement arqué, puis inégalement convexe-descendant à partir de l'extrémité du ligament postérieur. Région antérieure développée, bien ronde et légèrement décurrente à la base. Bord inférieur un tant soit peu convexe-descendant, ensuite parfaitement arrondi à son extrémité en remontant vers le rostre. Révẹing postérieure plus de deux fois plus longue que l'antérieure augmentant en hauteur jusqu'à 26 mill. $1 / 2$ en arrière de 


\section{$-261-$}

la perpendiculaire, et allant ensuite en s'atténuant, surtout dans son contour supérieur, pour se terminer en un rostre arrondi, aux trois quarts inférieur.

Sommets légèrement ventrus, assez convexes bien qu'à peine proéminents, sillonnés par des rides tremblotées. Crochets aigus. Arête dorsale bien accusée, : ensible seulement vers les sommets, et ornée de deux radiations foncées, divergentes jusqu'au rostre. Crête dorsale peu développée, comprimée vers l'angle postéro-dorsal, qui est arrondi-descendant et très émoussé.

Stries assez fines sur la convexité, puis devenant peu à peu plus fortes vers les contours. Épiderme très brillant, d'une coloraiion foncée d'un rouge-olivâtre passant au noir-olivàtre vers les contours et à la partie antérieure; enfin, offrant sur la région de l'arête dorsale des radiations foncées. Intérieur d'une belle nacre irisée de nuance blanche bleuacée, ou orangée sous les sommets.

Ligaments : antéro-interne occupant toute l'épaisseur de la cardinale; postérieur médiocre, presque entièrement recouverl. Lunule triangulaire. Charnière mince, légèrement ondulie sous les crochets.

J'ajouterai encore que, chez la glabra, la convexité bien régulière offre son maximum à peu de distance des sommets et de l'angl's postéro-dorsal.

\section{Anodonta Idrina.}

Anodonta Idrina, Spinelli. cat. Moll. prov. Bresciana, p. 19, f. 1-16, 1851 el (ze édil., 185̈6) p. 48, f. 1-16.

Très abondante dans le lac d'Idro, en Lombardie, où celte espice vil dans les endroits où le fond est sableux. 
Cette Anodonte est bien représentée dans le travail de Spinelli.

L'Idrina, figurée par Clessin (Anod, in : $2^{\bullet}$ édit. Chemnitz, pl. LV, fig. 1-2., 1876), est l'Anodenta Sebinensis d'Adami. Dans son texte (p. 168) Clessin, comme je l'ai dit plus haut, a confoudu, suus le nom d'Idrina, un grand nombre d'espèces différentes.

L’auteur des suites à Rossmässler (pl. CXX) a donné sous l'appellation d'Idrina (fig. 1156 à 1159) une série de formes distinctes. Parmi ces figures, celle qui se rapproche le plus de celle de Spinelli est la fig. 1157. J'ai exprimé (p.194) mon opinion sur chacune des formes représentées.

\section{Anodonta sublexata.}

Anodonta subluxata, Küster, Anod. in : éd., Chemnitz, p. 52, pl. XIII, f. 1-2 (seulement 1), 1832.

Je crois qu'il convient de rapporter à cette espèce !es figures 419 et 420 de l'Tconographie de Rossmässler, inscrites sous le nom erroné d'anatina. La furme représentée 419 provient d'un ruisseau, près de Chemnitz, en Saxe; celle 420 , d'un affluent de l'Elbe, près de Magdebourg, dans la Saxe prussienne.

Quant au type de Küster, il a été trouvé dans l'Altmühl, non loin de Ratisbonne, en Bavière.

1. La fig. 3 ne me parait pas ètre une subluxata. 


\section{Anodonta nycterina.}

Anodonta nycterina, Bourguignat (voir ci-dessus p. 104).

C'est cette forme qui a été présentée par le $\mathrm{S}^{r}$ Drouët, comme l'anatina type de Linuœus (Étud. Naïades France, pl. IV, fig. 1.)

La nycterina vit dans la Barse et dans divers ruisseaux aux environs de Troyes, dans le département de l'Aube.

Je connais encore cette Anodonte, de la Canne à SaintSaulge, dans la Nièvre, d'où je l'ai reçue sous le nom de variabilis; du lac des Quatre-Cantons, près de Kussnacht, en Suisse.

Il existe dans le Menthon (Ain) une variété à région antérieure un peu plus rétrécie (Locard).

\section{Amodonta Westeriundi.}

Anodonta Westerlundi, Fagot, in litt.

Petite espèce, de forme oblongue dans une direction descendante, caractérisée : par un ligament postérieur très court, terminé par une longue lunule; par une crête dorsale conme atrophiéc, sans angle postéro-dorsal défuni; par une convexité médiocre des plus régulières, etc.

Long $\max . \ldots \ldots \ldots \ldots \ldots \ldots \ldots .66$ mill.

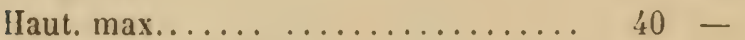

Epaiss. max. (à 18 dles sommels, à 40 du rostre, à 28 du bor $\mathrm{d}$ anlír., à 20 de 
l'angle postéro-dorsal, et du la base de la perpend.)...............

Long. de la crête liguam. dors., des sommets à l'angle postéro-dorsal 1 ......

Dist. de cet angle au rostre..........

Corde apico-rostrale..............

Haut. de la perpendiculaire........ 36 1/2

Dist. de cette perpend. au bord antér...

— du même point de cette perpend, au rostre................... 46 -

- enfin, de la buse de la perpend. à l'angle postéro-dorsal.........

21 mill.

$27-$

$33-$

50 -

$20-$

$38-$

Coq. à valves relativement épaisses, d'une teinte très foncée, assez grossièrement striées, båiilantes en avant et en arrière.

Bord supérieur légèrement arqué, jusqu'à l'extrémité du ligament, puis fortement convexe-descendant jusqu'au rostre. Région antérieure bien ronde. Bord inférieur régulièrement et faiblement convexe dans une direction un peu descendante. Région postérieure plus de deux fois plus longue que l'antérieure, augmentant en taille (de 3 mill. 1/2) jusqu'au niveau de l'extrémité du ligament, puis allant en s'alténuant jusqu'à un rostre assez obtus et arrondi.

Sommets déprimés, non saillants, malgré tout régulièrement convexes, presque toujours excoriés, et lorsqu'ils ne le sont pas, présentant de petites rides tremblotées très délicates. Crochets émoussés, comme obtus. Arête 
dorsale nulle, confondue dans la convexité. Crête dorsale également nulle.

Stries assez fortes, surtout grossières vers les contours et parfois feuilletées. Épiderme très foncé, d'un aspect terne, souvent encrassé, offrant tantôt un coloris uniforme d'une nuance terreuse, passant à un jaune orangébrique vers les sommets; tantôt une teinte noire-olivâtre vers les contours, s'éclaircissant sur la région ventrale en un ton rouge-brique. Intérieur d'une nacre blanchâtre peu irisée, prenant quelquefois un aspect bleuacé.

Ligaments: antéro-interne très épais, occupant tuute l'épaisseur de la cardinale; postérieur très court, saillant, terminé par une longue lunule.

Ruisseau de l'Yrette à Orsay, près de Paris (Coutagne). Gette espèce vit également à Shäne, en Suède. C'est l'Anodonta anatina, var. rostrata, envoyée de cette localité par le $D^{r}$ Westerlund.

\section{Anodonta Ogerieni.}

Cette Anodonte, à peu près de même taille que la précédente, diffère de la Westerlundi, en ce sens, que cette coquille est oblongue dans une direction moins descindante. Sa région postérieure, en outre, augmente moins jusqu'au niveau de l'exırémité du ligament, et son rosıre est plus largement obtus. Le bord inférieur est rectilignedescendant et non convexe. Le contour, des $\leqslant 0$ umets au rostre, offre une courbe arrondie d'une grande rigularitś (celui de la Westerlundi est d'abord arqué, puis, à partir de l'extrémité du ligament, où il éprouve un rectrait à l'endroit de la lunule, il descend sous une très forte inclinaison, tout en restait convexe). La crête dorsale, bien que 
médiocre, n'est pas atrophiće, comme celle de la précédente, elle est mẻme légèrement comprimée. La convexité est un peu plus supérieure; enfin, le ligament postérieur est moins court.

\begin{tabular}{|c|c|c|}
\hline $\mathrm{ax}$ & & nill. \\
\hline Haut. max.... & 31 & $1 / 2$ \\
\hline $\begin{array}{l}\text { Epaiss, max. (a } 15 \text { des sommets; à } 39 \text { du } \\
\text { rostre; à } 28 \text { du bord antér.; à } 19 \text { de } \\
\text { l'angle postéro-dorsal; à } 23 \text { de la base } \\
\text { de la perpendiculaire)............. }\end{array}$ & 21 & - \\
\hline $\begin{array}{l}\text { Long. de la crête ligam.-dorsale, des som- } \\
\text { mets à l'angle postéro-dorsal......... }\end{array}$ & 28 & - \\
\hline Distance de cet angle au rostre......... & 32 & - \\
\hline Corde apico-rostrale............... & 5 & - \\
\hline Haut. de la perpendiculaire........... & 35 & - \\
\hline $\begin{array}{l}\text { Dist. de cette perpend. au bord antér... } \\
\text { — du même point de cette perpend. au }\end{array}$ & 19 & - \\
\hline - enfin, de la base de la perpend. à l'an. & 47 & \\
\hline gle postéro-dorsal............ & 39 & \\
\hline
\end{tabular}

Coq. ì valves médiocrement épaisses, oflrant deux bâillements peu ouverts, l'un en avant, l'autre en arrière.

Bord supérieur presque rectiligne au niveau des sommets, puis présentant une courbe bien arrondie jusqu'au rostre. Région antérieure exactement ronde. Bord inférieur rectiligne-descendant, brièvement recourbé-remontant à son extrẻmité. Région postérieure deux fois et demi plus longue que l'antćrieure, augmentant insensiblement jusqu'au niveau de l'extrémité du ligament postérieur, s'atténuant sous la forme d'une large partie rostrale. 
Sommets comprimés, comme aplatis, hicin que régulièrement convexes et ne donnant lieu à aucune saillie, sillonnés de fortes rides espacées, tremblotées. Crochets faiblement aigus. Arête dorsale peu accusée, confondue dans la convexité des valves, ordinairement ornée de deux radiations vertes divergentes. Crête médiocre, légèrement comprimée, sans angle prononcé.

Stries régulières, plus ou moins délicates, un peu plus fortes vers les contours. Épiderme brillant, d'un beau coloris, tantôt d'une nuance uniforme olivâtre avec quelques radiations plus foncées, tantôt d'une teinte jaunacéeverdâtre, passant au rouge vers les sommets, et surchargée sur la région postérieure de radiations bien vertes. Intérieurd'une nacre blanche-bleuacée, peu irisée.

Ligaments : antéro-interne filiforme; postéricur peu allongé, à moitié recouvert. Lunule longue, subtriangulaire.

Cette espèce, à laquelle j'attribue le nom du frère Ogé rien, ancien directeur des Écoles chrétiennes de Lons leSaulnier, et auteur d'une bonne faune du Jura, habite aux environs de Marboz et dans le Suran, près Gigny, dans I Jura.

\section{PQD ACALUIANA}

Les Anodontes de ce groupe sont de formes allongée:, à bords (supérieur et inférieur) presque parallèles. Les valves, jamais lisses et brillantes, d'une teinte sombre, et sillonnées par des stries grossières, souvent rugueuses, sont généralement excoriées ou fortement encrassées. Les sommets (sauf chez la Potiezi) sont peu convexes, souvent 
aplatis et comprimés. La région postérieure très allongrée, augmente fort peu en hauteur.

Les Acalliana vivent dans les eaux peu courantes, à fond de graviers.

\section{Anodonta Ervica.}

Long. $\max . . \ldots \ldots \ldots \ldots \ldots \ldots \ldots \ldots . . .65$ mill.

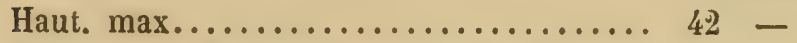

Épais. max. (à 25 des sommets, à 4 so du rostre, à 41 du bord antér., à 23 de l'angle postéro-dorsal, à $271 / 2$ de la base de la perpend.)..................... 29 -

Long. de la crête ligam.-dors., des sommets à l'angle postéro-dorsal............ 3ร̆ -

Distance de cet angle au rostre......... 37 -

Corde apico-rostrale............... 70 -

Haut. de la perpendiculaire.......... $401 / 2$

Dist. de cette perpend. au bord antér..... 20ั -

- du même point de cette perpend. au rostre..................... 60 1/?

- enfin, de la base de la perpend. à l'angle postéro-dorsal............. 50 -

La convexité, presque centrale à quelques millimètres près, est régulière, sauf entre l'arête et la crête dorsale, où elle descend rapidement. Les valves, assez épaisses, fortement bâillantes en arrière et surtout en avant, sont grossières comme rugueuses, avec un épiderme d'une teinte uniforme très foncé, mais le plus souvent profondément excorié.

Coq. d'une forme oblongue-allongée dans une direction 
presque horizontale, malgré tout un tain suit peu inclinée, et possédant des bords (supérieur et inférieur) presque parallèles.

Bord supérieur légèrement convexe, puis fortement descendant, à partir de l'angle postéro-dorsal. Région antérieure bien ronde. Bord inférieur faiblement subrectiligne, un tant soit peu rentrant à sa partie moyenne, ensuite très peu remontant à son extrémilé. Région postérieure, près de deux fois et demie plus longue que l'antérieure, allant à peine en augmentant jusqu'au niveau de l'extrémité du ligament postérieur, puis s'atténuant d'une façon brusque, surtout supérieurement à partir de l'angle, pour se terminer par un rostre obtus et inférieur.

Sommets (toujours profondément excoriés) subventrus et faiblement convexes. Arête très obtuse, émoussée, peu définie, malgré tout, bien accusée par la convexité prononcée de cette partie, par suite de l'inclinaison rapide du test sur la crête dorsale, qui est presque nulle et à peine comprimée.

Stries grossières, rugueuses, comme imbriquées. Ėpiderme assez terne, foncé, d'une teinte uniforme d'un marron-feuille-morte; passant vers l'extrémité rostrale à une nuance jaune, puis verte très prononcée. Intérieur d'une nacre sans éclat, blanchâtre, avec des taches jaunacées livides.

Ligaments : antéro-interne épais, très allongé en avant sur le contour antérieur; postérieur court, très puissant et fort saillant. Lunule triangulaire. Charnière ondulée, sans éminences bien accusées. Impressions profondes.

La rivière de l'Erve, au-dessous du château de Thevalles, près Chéméré-le-lioy, dans la Mayenne, où elle est abondante. 


\section{Anodonta Carisiana}

Anodonta Carisiana, Mabille, in litt.

Cette espèce, plus petite que la précédente, d'une forme moins allongée, offre une direction descendante accusée; ses valves minces, légères, sont très bâillantes en avant et en arrière; ses sommets non ventrus, sont comme écrasés; sa convexité n'est pas régulière.

Long. max................ 70 mill.

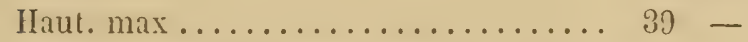

Épaiss. max. (à 25 des sommets, à 36 du rostre, à 32 du bord antér., à 20 de l'angle postéro-dorsal, et de la base de la perpendiculaire.)............

Long. de la crête ligam.-dors., des somm. à l'angle postéro-dorsal.......... 28 -

Dist. de cet angle au rostre......... 35 -

Corde apico-rostrale............. ॐ99 -

Haut. de la perpendiculaire......... 35 -

Dist. de la perpend. au bord antér..... Is -

- du même point de cette perpend. au rostre .............. छl -

- enfin, de la base de la perpend. à l'angle postéro-dorsal ....... 40 -

Bord supérieur légèrement arqué, puis descendan d'une façon presque rectiligne sur le rostre à partir de l'angle. Région antérieure très courle, bien ronde. Bora' inférieur régulièrement et très faiblement convexe dans MAI. 1881. 
toute son étendue. Région postérieure, près de trois fois plus longue que l'antérieure, allant en augmentant fort peu en hauteur (de 4 mill.) jusqu'à 22 mill. en arrière de la perpendiculaire, et s'atténuant ensuite sous la forme d'un rostre arrondi, moins obtus et moins inférieur que celui de l'Ervica.

Sommets écrasés, non ventrus ni saillants, toujours excoriés, néanmoins laissant voir sur la couche sous-épidermique le relief de fortes rides espacées, régulières, non tremblotées, mais droites, s'arrêtant brusquement sur la partie où commence l'arête dorsale. Cette arête très obtuse est fortement saillante dans toute son étendue. Grête peu développée et médiocrement comprimée, sans angle saillant à son extrémité.

Stries fortes, çà et là saillantes et rugueuses, enfin, comme imbriquées vers les contours postérieurs. Épiderme terne d'une teinte foncée uniforme d'un cendrénoir-olivâtre. Intérieur d'une nacre bien irisée à reflets bleuâtres.

Ligaments : antéro-interne très en avant ; postérieur médiocre. Lunule allongée. Charnière ondulée, avec une petite éminence tuberculeuse sous les crochets.

Ganal du Gher, à Tours (Mabille).

\section{Anodonta Potiezi.}

Anodonta coarctata 1, Potiez et Michaud, Moll. gal. Douai, II, 1844, p. 142, pl. LV, fig. 2.

Cet Acéphale a été considéré à tort comme semblable à

1. Non, An. coarctata, Anton (Verz. conch., p. 16, n० 583, 1839), espèce différente du Mexique. 
la parvula, espèce écourtée, obtusément subirigone, à crête dorsale comprimée et assez accentuée.

La Potiezi (coarctata de Potiez et Michaud1) est une forme ovale-allongée; à sommets saillants, gros, trèz obtus; à bord inférieur sinué à la partie médiane. D'après la gravure, la région postérieure augmente peu en hauteur; la partie rostrale parait arrondie-subanguleuse; les stries semblent grossières; les bords (supérieur et inférieur) sont un tant soit peu parallèles; sa taille atteint 25 à 30 en longueur, 15 à 20 en hauteur, et 10 à 12 en épaisseur.

Je ne connais pas cette Anodonte, qui est signalée des eaux courantes de la Franche-Comté.

\section{Anodonta Klecaki.}

Anodonta Klecaki, Drouët, in : Journ. conch. 18s1, p. 28.

Primilivement confondue avec la parvula, elle a été, avec raison, distinguée dernièrement de cette espèce et dédiée au malacologiste Dalmate Biagio Klecak.

Gette Anodonte est abondante près d'Obbrovazzo, dans la Krupa, affluent de la Zermanja, en Dalmatie.

\section{Anodonta exulcerata.}

Anodonta exulcerata, Villa, in : C. Porro, Malac. prov. comasca, p. III, pl. 2, f. 12, 1838.

La figure donnée par Porro représente bien les contours de cette espèce allongée, peu ventrue, à bords

1. Non coarctata des autres auteurs. 
(supérieur et inférieur) presque parallè'es. J'en possède, des environs de Milan, des échantillons tout à fait semblables.

Clessin (Anod. in : $2^{\circ}$ édit. Chemnitz, p. 127, pl. XL, f. 3-4, 1876) a décrit et fait figurer comme l'exulcerata type de Villa, une Anodonte toute différente, à crête dorsale accentuée; à bords non parrallèles; à valves très bombées, qui me paraît être une forme bien ventrue de la Benacensis. Clessin donne pour patrie à son exulcerata, à laquelle il ratlache l'Anod. gibba de Kutschig, la Dalmatie et le nord de l'Italie. Il est plus que probable que cet auteur a confondu deux espèces : la gibba ${ }^{1}$ de Kutschigg, espèce inédite, qui m'est inconnue, et, la Benacensis de Lombardie qu'il a prise pour l'exulcerata, bien que page 168 , il ait considéré la vraie Benacensis comme une variété de l'Idrina.

L'exulcerata de Villa vit dans les petits lacs d'Oggiono, d'Alserio et de Pusiano près de Brianza, en Lumbardie.

\section{Anodonta acallia.}

Anodonta acallia, J. Ray, in litt.

Cette jolie petite Anodonte est bien rendue dans les suiles à Rossmassler (fig. 165̃9) sous le nom erroné de coarctata.

L'auteur dit qu'il a reçu son espèce de l'abbé Dupuy, comme la véritable parvula de Drouët. Cette nouvelle erreur prouve derechef qu'il ne faut accepter aucune détermination sans vérification. Si cet auteur avait pris

1. Non, gibba de Held, qui est ma cyrtoptychia (voir ci-dessus page 136, nec, gibba de Benson, $185 \tilde{\text { J }}$, espèce d'Asie). 
cette précaution, il aurait vu : $1^{\circ}$ que la parvula, telle qu'elle est figurée (Etud. Naïad. France, pl. IV, f. 2), est une petite espèce écourtcee, subtriangulaire; $2^{\circ}$ que la coarctata de Potiez et Michaud représentée (Moll. gal. Douai, pl. LV, f. 2) est une Anodonte ventrue, plus haute, moins allongée, à bord inférieur sinué, à sommets gros, obtus et saillants.

L'honorable abbé Dupuy, qui a envoyé à l'auteur dıs suites à Rossmassler, cette parvula des environs de Troyes, Ia tenait du sieur Drouët, qui, à cette époque, appelait parvula, tout ce qui était petit, sans s'inquiéter si ses échantillons étaient allongés, aplatis, ventrus, écourtés ou subtriangulaires.

Ce qui prouve l'amalgame des formes, est la description même donnée par cet auteur, description qui ne concorde pas très bien avec les caractères de la parvula qu'il a fait figurer. Je dis, ne concorde pas très bien, car il est difficile desaisir la pensée d'un auteur qui prend le devant pour le derrière, ou le derrière pour le devant, en un mot, qui ne sait où se trouvent les côtés antérieur et postérieur. Actuellement, ce même auteur, par un motif incompréhensible, appelle, dans les descriptions qu'il vient de publier sous le titre d'Unionido nouveaux (Journ. Conch., 1879 et 1881), appelle, dis-je, concave, ce qui est convexe, et convexe, ce qui est concave. G'est tout simplement insensé! On vou. drait à plaisir jeter une obscurité profonde sur les espèces d'Anodontes, déjà si difficiles à distinguer, qu'on ne saurait mieux faire. C'est le comble de l'aberration!

Pour en revenir à cette coarctata que l'abbé Dupuy a envoyée à l'auteur des suites à Rossmassler, coarctata qui a été prise par l'auteur allemand comme "pain béni », je ferai remarquer que l'abbé Dupuy (Hist. moll., pl. XX, 
f. 21) a représenté une coarctata troyenne, non seulement différente de celle qu'il a envoyée, mais encore dissemblable du type figuré par le très savant sieur Drouët. Que l'on compare les figures, on verra si je ne dis pas la vérité. En somme, avec le système de la vieille école, qui consiste à tout réunir, on arrive à ne plus savoir ce qu'est le type d'une espèce. Pour elle, tout ce qui est petit est naturellement parvula, tout ce qui vit dans l'eau est fluviatilis (ex. ancylus fluviatilis), tout ce qui habite les fontaines est fortinalis (ex. cyclas (Pisidium) fontinalis), et, ainsi de suite.

Pour moi, qui m'honore de ne pas appartenir à cette école d'ignorance, je n'admettrai jamais qu'une espèce puisse être ou allongée, à bords (supérieur et inférieur) parallèles, à sommets écrasés, comme la coarctata représentée (fig. 1659) dans les suites à Rossmassler; ou subtrigone-descendante, à stries ellipsoïdes, etc., comme cette autre coarclata figurée ( $\mathrm{pl}$. XX, f. 21) dans l'ouvrage de l'abbé Dupuy; ou enfin, écourtée-subtrigone, à bords (supérieur et inférieur) divergents, à région postérieure très dilatée et très obtuse, comme cette coarctata, métamorphosée en parvula, dans les Études sur les Nä̈ades de France (pl. IV, f. 2).

La première est l'acallia de J. Ray,

La seconde la podica d'Émile Pillot,

La troisième reste la parvula de Drouët.

Aucunes ne sont la coarclata de Potiez et Michaud, coarctala qui doit prendre dorénavant le nom de Potiezi, pour éviter le double emploi des noms.

Je passe maintenant aux caractères de l'acallia.

Long. max..................

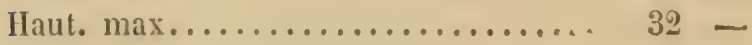


Épaiss. max (à 18 des sommets; à 31 du rostre; à $: 8 \mathrm{du}$ bord antér.; à $18 \mathrm{de}$ l'angle postéro-dorsal; à 17 de la base de la perpend.)...............

Long. de la crête ligam.-dors., des sommets à l'angle postéro-dorsal........

Distance de cet angle au rostre........

Corde apico-rostrale................

Haut. de la perpendiculaire..........

Dist. de cette perpend, au bord antér...

- du même point de cette perpend. au

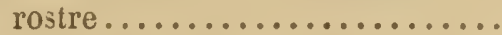

16 mill.

$27-$

27 -

11 -

$28-$

$131 / 2$

$421 / 2$

- enfin, de la base de la perpend. à l'angle postéro-dorsal.......... 33 -

Je ferai remarquer que l'angle postéro-dorsal est à égale distance des sommets et du rostre, puisqu'il y a 27 mill. d'un côté comme de l'autre; que le point maximum de la convexité, point pour ainsi dire central (à 3 mill. près), est aussi distant des sommets que de l'angle postéro-dorsal, ainsi que de la base de la perpendiculaire à un millimètre près.

L'acallia de J. Ray est une petite Anodonte assez com. primée, très peu convexe, de forme allongée, à bords (supérieur eí inférieur) presque parallèles. Ses valves minces, légères, fragiles, assez grossièrement striées, d'une teinte uniforme assez claire, offrent deux bâillements : un très ouvert, depuis le commencement supérieur de la région antérieure jusqu'à la partie rostrale; l'autre beaucoup plus étroit, du rostre à l'angle postéro-dorsal.

Bord supérieur rectiligne, puis, à partir de l'angle, convexe-descendant jusqu'au rostre. Région antérieure 
exiguë, bien arrondie. Bord inférieur subrectiligne, dans une direction faiblement descendante et un peu convexeremontant à son extrémité, Région postérieure, plus de trois fois plus développée que l'antérieure, allongée en augmentant en hauleur jusqu'au niveau de l'extrémité du ligament, puis s'atténuant (surtout supéric urement), pour' se terminer par une partie rostrale inférieure, comprimée, tris obluse et arrondie.

Sommets aplatis, à peine converes, comme écrasés, sillonnés par de petites rides régulièrement concentriques. Crochets aigus. Arête dorsale nulle, confondue dans la convexité. Crête médiocre, un peu comprimée vers l'angle.

Stries relativement fortes; régulières sur la région ventrale, devenant grossières et même feuilletécs vers le contour inféro-antérieur et imbriquées vers le bord postérieur. Épiderme assez terne (presque toujours encrassé), d'une teinte uniforme d'un ton oliveâtre pâle, ou cendréjaunacé, s'éclaircissant vers les sommets. Intérieur d'une nacre bleuacéc bien irisée.

Ligaments : antéro-interne très antérieur, relativement robuste; postérieur allongé, assez saillant, d'une belle teinte marron. Lunule étroite, très allongée.

L'acallia vit dans la Laignes aux Riceys (département de l'Aube). Je ne la connais pas des environs de Troyes.

\section{Anodonts illora.}

Anodonta illota, J. Ray, in litt.

Cette espèce, qui vit également dans la Laignes aux Riceys (Aube), est une forme que J. Ray de Troyes a eu raison de distinguer. 
Cette forme est caractérisée par un test moins allongé; par une région postérieure plus écourtée, plus descendante, terminée par une partie rostrale inférieure, regardant en bas et moins obtuse; par une région antérieure plus développée, par cela même que les sommels sont plus médians; surtout par sa crête dorsale plus courte, dont l'angle postéro-dorsal est saillant et carrément coupé. Chez cetle espèce, le contour, entre l'angle et le rostre, en descendant subitement d'une façon rectiligne, donne à cette coquille une apparence tronquée très accentuéc. Chez elle encore, les bords (supérieur et inférieur) sont moins parallèles.

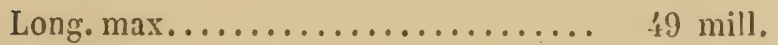

Haut. $\max . . . . . . . . . . . . . . . . . .30$ -

Épaiss. max. (à 13 des sommets, à 28 du rostre, à $23 \mathrm{du}$ bord antér., à $14 \mathrm{de}$ l'angle postéro-dorsal, à 17 de la base de la perpend.).............. 15 -

Long. de la crête ligam.-dors., des sommets à l'angle postéro-dorsal...... 20̈ -

Dist. de cet angle au rostre......... 26 -

Ciorde apico-rostrale............. $40-$

Haut. de la perpendiculaire......... 26 -

Dist. de la perpend, au bord antér..... 15 -

- du même point de celte perpend. au rostre................ 34 -

- enfin, de la base de la perpend. à l'angle postéro-dorsal....... 30 -

Bord supérieur presque rectiligne, très faiblement incliné jusqu'à l'angle, ensuite descendant en ligne droite 
(sous un angle de $60^{\circ}$ ) sur le rostre. Région antérieure arrondie, relativement développée. Bord inférieur bien descendant, faiblement arqué. Région postérieure un peu plus de deux fois (et non plus de trois fois, comme celle de l'acallia) plus longue que l'antérieure, augmentant en hauteur' jusqu'au niveau de l'extrémité du ligament, ensuite s'atténuant subitement à partir de l'angle postérodorsal, pour se terminer par un rostre tout à fait inférieur arrondi et peu obtus.

Sommets écrasés, aplatis (plus distants du bord antérieur que ceux de l'acallia), et sillonnés de petites rides très tremblotées. Grochets très aigus. Arête dorsale nulle. Crête plus élevée et plus comprimée à l'angle postérodorsal cue celle de la précédente.

Stries, coloration et nacre à peu près semblables à celles de l'acallia.

Ligament ¿postérieur court. Lunule triangulaire, non allongée.

\section{A MEATEANA}

Les Arealiana, dont la plupart ont été prises, ainsi que les Westerlundiana, pour des anatina, sont des espèces oblongues, allongées, à crête dorsale plus ou moins développée et comprimée, suivant les espèces. L'angle postéro. dorsal, à l'exception de quelques-unes, est nettement coupé et fortement anguleux. En somme, ces Anodontes sont relativement très hautes pour leur longueur. Elles vivent indifféremment dans les lacs et les rivières à fond vaseux. 


\section{Anodonta arealis.}

Anodonta arealis, Kruster, Anod. in : $2^{\circ}$ édit. Chemnitz, p. 47, pl. IX, f. 2-4, 1852 .

Anodonta emeria, Bourguignat, mss. olim.

Espèce très bien représentée dans le travail de Kuster.

L'arealis vit dans le lac de Morat, en Suisse, non loin de Faouy. Je l'ai recueillie bien typique dans le lac du Bourget, en Savoie. Je la connais encore de la Masle à Amboise (Mabille).

\section{Anodonta subarealis.}

Anodonta subarealis, Fagot, in litt.

G'est l'Anodonta anatina de l'abbé Dupuy (Hist. moll. France, pl. XIX, f. 13). La figure donnée par Dupuy est excellente et suffit pour la connaissance de cette espèce. On n'a, en effet, qu'à comparer cette figure avec celles de l'arealis de Küster (pl. IX, f. 2-4), ou de l'anatina (fig. 417 de l'iconographie de Rossmassler) pour voir de suite les grandes différences qui existent entre ces Anodontes.

C'est encore l'Acéphale représentẻ (fig. 1960 dans les suites à Rossmassler) sous le nom erroné d'Anod. Scaldiana. La Scaldiana, en effet, comme je l'ai dit plus haut, (pages 238-241) est bien dissemblable.

La subarealis vit çà et là dans les rivières de France. Je la connais de l'Escaut à Valenciennes, de la Seine, et, notamment de divers cours d'eau des environs de Dax, 
dans les Landes. Les échantillons 'de cette dernière localité me sont parvenus tantôt sous le nom de piscinalis, tantôt sous celui de Moulinsiana.

Je suis assez tenté de rapprocher de la subarealis, toute. fois à titre de variété, une petite forme de Castelgoffredo, en Italie, forme qui m'a été adressée, sous l'appellation de piscinalis par M. Raimondo del Prete.

Chez cette Anodonte, la crête, faiblement développée, est peu comprimée; la convexitó un peu plus accentuée parait plus bombée, notamment sur la région de l'arête.

\section{Anodonta Kusteri.}

J'inscris sous ce nom une forme du groupe des Arealiana, que Küster (Anod. in : $2^{\circ}$ édit. Chemnitz, pl XIs, fig. 3 sculement ${ }^{1}$ ) a représenté sous le nom de ventricosa.

Cette ventricosa de Küster, qui ne ressemble en rien à celle de C. Pfeiffer, se distingue de l'arealis par sa forme moins haute (4't mill, contre כૅ mill. chez l'arealis), par conséquent ayant une apparence plus allongée; par sa crète moins longue (long. 29 mill.; velle de l'arealis a 35 mill.), moins développée, moins comprimée, avec un angle postéro-dorsal non aussi aigu ni aussi carrément coupé ; par son arête pourvue da deux zones vertes divergentes, descendant sur le rostre sous une inclinaison plus rectiligne (celle de l'arealis possède deux sillons étroits, divergents, un tant soit peu saillants et moins rectilignes); par sa région antérieure mieux arrondie et moins décurrente à la base; par son bord inférieur peu arqué, à peine

1. La fig. 5 doit être altribuée à une autre forme du groupe de la Glyca. 
remontant à son extrémité (celui de l'arealis, bien arqué, est fortement convexe-remontant vers la partie rostrale); par sa région postérieure plus allongée, moins haute terminée par un rostre plus inférieur, etc...

La Kusteri habite les cours d'eau du sud de Wurtemberg.

\section{Anodonta Morchiana.}

Anodonta mutabilis, var. Mörchiana, Clessin, Anod. in : 2 édit. Chemnitz, p. 238, 1876, et Anodonta Morchiana (même ouvrage) explic. des pl., p. 280, pl. LXXVII, f. $1-2$.

Espèce bien rendue dans le travail de Clessin. Cet auteur signale cet Acéphale du Danemark, des environs de Glimsö, dans l'île Seeland.

Mörch m’a envoyé, de Tüssủ et d'une autre localité dont je n'ai pu déchiffrer le nom, celte même Anodonte, sous l'appellation de macula.

Je la connais encore de Chalon-sur-Saône (Fagot).

\section{Anodonta maculata.}

Mytilus macula, Sheppard, on two new Brit. spec. of Mytilus, in : Linn. trans. XIII, 1820, p. $83, \mathrm{pl}, \mathrm{V}, \mathrm{f} .6$, et (traduct. Chenu), p. 270, pl. XIII, f. 3, 1845.

Petite espèce, très voisine de la Mörchiana, en différant notamment par sa crête plus développée, plus comprimée; par son angle postéro-dorsal presque aigu et car- 
rúment coupé (l'angle de la Mörchiana, très obtus, est à peu près nul); par son test un peu moins convexe, etc.

Abondante dans les canaux des jardins de Campsey-ash, en Angleterre, et, dans le Morin, à Esbly, dans Seine-etMarne (Mabille). Je l'ai reçue de Mőrch, du Danemark, sous son vrai nom de macula (mieux maculata).

Le nom de macula lui a été donné, parce que tous les échantillons de Campsey-ash étaient caractérisés par une large tache pourprée s'étendant sur les deux tiers de la surface externe, tache, ajoute Sheppard, qui pouvait être effacée par le frottement.

Les échantillons du Danemark sont sans tache. Malgré ce signe distinctif, qui n'en n'est pas un, puisqu'il peut s'effacer, je crois que cette Anodonte mérite d'être conservée.

Je renvoie pour la connaissance de cette espèce à la figure donnée par Sheppard.

\section{Anodonta Arelatensis,}

Anodonta Arelatensis, Jacquemin, guide du voy. dans Arles, p. 124, 1835, et, Dupuy, Hist. moll. France (6॰ fasc. 1852), p. 611, pl. XIX, f. 14.

L'abbé Dupuy rapporte à l'Arelatensis, l'Anodonta ovalis de Requien, coquille inédite, citée par Potiez et Michaud (Moll. gal. Douai, Il, p. 145, 1844). Je ne sais si ce rapprochement est juste, n'ayant jamais ru cette espèce, dont il n'existe aucune description.

L'Arelatensis habite l'étang de Meyranne, dans la Crau, au-dessous d'Arles, où elle a été découverte par notre ami feu Faysse. Je l'ai recueillie également près d'Arles dans 
le canal de Bouc. La figure donnée par Dupuy est bonne et rend bien l'aspect de cet Acéphale.

\section{Anodonta Benacensis.}

Anodonta Benacensis, Villa, in : Bull. malac. ital., 1871, p. 94 (sans descriptions).

Cette espèce a été représentée par Clessin (Anod. in : $2^{\circ}$ édit. Chemnitz, pl. XL, f. 3-4), sous le nom erroné d'exulcerata. L'échantillon figuré, bien que ventr'u, rend parfaitement la forme et l'aspect de cette coquille.

Elle vit, en Lombardie, dans les cours d'eau des environs de Bergame.

\section{Anodonta Clessini.}

Cette Anodonte, à laquelle j'attribue le nom de Clessin, a été figurée sous l'appellation d'anatina par cet auteur (Anod. in : $2^{\circ}$ édit. Chemnitz, pl .XIV, fig. 3 et $4^{1}$ ). Elle provient des environs de Krakow, dans le Mecklembours. Elle vit également dans le lac de Genève, d'où Brot l'a adressée au professeur Deshayes, sous le nom d'anatina.

Cette espèce, très caractérisée, est si bien reproduite, qu'il est inutile de donner ses signes distinctifs. Elle pourra toujours être distinguée et reconnue au moyen des figures que je viens de signaler.

1. Les analina représentées pl. XXVI, f. 1-2, sont des formes très différentes et de l'anatina (type) et de celle-ci. 


\section{Anodonta pàruila.}

Anodonta parvula, Drouët, Etud. Anod. de l'Aube (art. 2) p. 9, pl. IV, f. 2, 1852 (sous le titre d'Études sur les Nayades de la France).

C'est à tort que l'auteur de cette espèce a rapporté sa parvula à la coarclata de Potiez el Michaud. Cette coarctala, ainsi que je l'ai dit ci-dessus (page $\dot{2} 74$ ), est un Acéphale diflérent de celui-ci.

La figure 2 de la planche IV des Naïades de France rend très exactement le port et l'aspect de cette espèce écourtée, subtrigonale, à région postérieure très dilatée en hauteur et terminée par une parlie rostrale excessivement obtuse. Cette figure, si l'on veut bien l'étudier, suffit amplement à la connaissance de la parvula. Je n'en dirai pas aulant de la description qui n'est pas exacte.

Le type provient de l'Ourse à Bar-sur Seine, ainsi que de divers cours d'eau (dérivations de la Seine) en amont de la ville de Troyes.

Je connais encore la parvula des localités suivantes:

Canal de l'Ourcy à Bondy et à Neaux; ruisseau à Brunoy, et, l'Orge à Juvizy, dans Seine-et-Oise (Mabille); ruisseau de Longpoint, dans l'Aisne (Mabille); la Nonge it Laigé, dans Saône-et-Loire (Locard); Ia Masle à Amboise, le caral de Loir et-Cher, près de Tours (Rambur); le Maine à Angers (Servain), etc.

\section{Anodonta pocalica.}

Anodonta podica, E. Pillot, in litt.

C'est cette espèce qui a été figurée par l'abbé Dupuy 
(Hist. moll. France, pl. XX, f. 21) sous le nom de coarctata. Celte figure rend très bien la physionomie de cette Anodonte.

La peedica est une petite forme oblongue dans une direction descendante, à crête peu prononcée, et, dont l'angle postéro-dorsal est si émoussé et si obtus, qu'il est presque nul. La région postérieure, très allongée, se termine par un rostre inférieur obtus-arrondi. Le bord supé. rieur offre une cuurbe presque parfaite jusqu'au rostre et l'inférieur est médiocrement arqué-descendant. Chez celte espèce, les stries, délicates, sont sensiblement ellipsoïdes.

La podica, qui a été distinguée avec raison par Emile Pillot, habite dans la rivière de la Vacherie, près de Truyes (Aube).

\section{Anodonta codiella.}

La codiella est la plus pelite Anodonte que je connaisse. Elle ressemble à une petite boule, ou à un noyau de pêche de taille médiocre. Elle n'a que 36 mill. de longueur maximum. La poedica atteint 49 ; la parvula 48 à 522. Elle est remarquable par sa convexité ( 15 mill.) relativement forte pour sa grandeur, convexité très régulière, dont le point maximum est juste central. Sa hauteur $(26$ 1/2) est également très grande par rapport à sa taille.

Long. $\max . \ldots \ldots \ldots \ldots \ldots \ldots$

Haut. $\max \ldots \ldots \ldots \ldots \ldots \ldots \ldots$

Épaiss. max. (à 13 des sommets; à 18 du rostre et du bord antérieur, enfin, HAT. 1881. 
à 12 de l'angle postéro-dor'sal et de la base de la perpendiculaire)........ Long. de la crête ligam. dors., des sommets à l'angle pustéro-dorsal...... 17 -

Dist. de cet angle au rostre......... 18 -

Corde apico-rostrale............. 31 -

Hauteur de la perpendiculaire....... 23 -

Dist. de la perpendicul. au bord antér. 11 -

- du même point de cette perpend. au rostre.................

- enfin, de la base de la perpend. à l'angle postéro-dorsal...... $24 \quad-$

Bord supérieur faiblement arqué, puis, à partir de l'angle, descendant presque à pic, tout en présentant un contour légèrement courbe jusqu'au rostre. Région antérieure exiguë, arrondie, décurrente à la base. Bord inférieur convexe-descendant, remontant à peine à son extrémité. Région postérieure, un peu plus de deux fois plus longue que l'antérieure, allant en augmentant jusqu'au niveau de l'extrémité du ligament, puis s'atténuant subitement, surtout supérieurement, pour se terminer par une large partie arrondie excessivement obtuse, présentant à sa base un contour rostro-anguleux très émoussé.

Sommets à peine saillants, un tant soit peu bombés, sillonnés par de belles rides régulières, espacées, très faiblement tremblotées. Arîte confondue dans la convexité. Crête médiocre, obtuse, non comprimée, sans angle saillant.

Stries fines et régulières sur la région ventrale, devenant plus fortes vers les contours, et, comme imbriquées 
vers le bord postérieur. Epiderme sombre et terne, d'une teinte uniforme opale-noirâtre, s'éclaircissant sur les sommets, qui sont ordinairement excoriés. Intérieur d'une nacre terne bleuacée.

Ligaments: antéro-interne robuste, occupant toute l'épaisseur de la cardinale ; postérieur saillant, marron, relativement fort. Lunule filiforme. Impressions bien spathuliformes.

Cette charmante petite Anodonte habite dans la rivière de la Vacherie, près de Troyes (Aube), où je l'ai recueillie.

\section{ABRHEVIATIANA}

Les Abbreviatiana sont des Anodontes d'une forme oblongue dans une direction descendante, parfois très exagérée, comme chez l'eunotaia, caractérisées, en outre, par une crête dorsale et un angle postéro-dorsal nuls, comme atrophiés. Les valves d'une teinte sombre uniforme, sont chargées de stries rugueuses, sauf chez une espèce, et, la plupart du temps, sont excoriées ou encrassées d'un limon très tenace.

Ces Acéphales vivent dans les lacs ou les cours d'eau à fond de graviers.

\section{Anodonta Racketti.}

Cette Anodonte a été parfaitement représentée:

$1^{\circ}$ par W. G. Maton et Th. Rackett (a Desc. cat. of the Brit. test. in: Linn. trans. VIII, 1807, pl, III, f. 3 - et (traduct. Chenu), vol, des trans. de la Soc. linn. de Londres, pl. XVI, f. 3) sous le nom de Mytilus Cygnceus, Var. 
- par Th. Brown (lllust. land and freshw. Conch. great Brit. and Ireland, pl. XV, f. 1 (seulement), 1845), sous l'appellation d'Anodon ponderosa, var.

Cette singulière espèce, qui vit en Angleterre et en Éco se (notamment à Loch-end près d'Édimbourg), a été recueillie par M. Paul Fagot dans le canal du Midi à Villefranche-Lauraguais. Les échantillons du canal du Mili sont identiques à ceux de la Grande-Bretagne.

\section{Anodonta Carotae.}

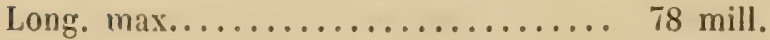

IIaut. $\max . \ldots \ldots \ldots \ldots \ldots \ldots \ldots \ldots$ \& $441 / 2$

Épaiss. max. (à 29 des sommets, à 38 du rostre, à 46 du bord antérieur, à 15 de l'angle postéro-dorsal, à 30 de la base de la perpend.).................

Long. de la crête ligamento-dorsale, des sommets à l'angle postéro-dorsal ..... 3̋ -

Dist. de cet ang'e au rostre........... 39 -

Corde apico-rostrale.............. 68 -

Haut. de la perpendiculaire........ \& 41 1/2

Dist. de cette perpend. au bord antérieur. 23 -

- du même point de cette perpend. au rostre.................. 57 -

- enfin, de la base de la perpend. à l'angle postéro-dersal........ 4 š -

D'après ces mesures, on remarque que la convexilé maximum n'est qu'à 15 mill. de l'angle postéro-dorsal, et, qu'elle est plus rapprochće du rostre que du bord antérieur. 
Cette espèce remarquable par son contour supérieur offrant une courbe parfaite des sommets au rostre; par sa crête nulle; par son angle postéro-dorsal complètement atrophié; par son arête présentant une courbe semblable à celle du contour supérieur, etc... Les valves, à peine bâillantes en avant et 'en arrière, rugueuses, d'un aspect terne et d'une teinte foncée, sont comprimées en avant, et notamment, vers le bord palléal, où la coquille éproave une légère contraction, qui donne lieu à une faible sinuosité.

Bord supérieur offrant une courbe parfaite dans toute son étendue. Région antérieure arrondie, assez largement développée. Bord inférieur un tant soit peu rectilignedescendant, avec une légère sinuosité en arrière de la perpendiculaire. Région postérieure allongée dans une direction descendante, près de deux fois et demie plus longue que l'antérieure, et, ayant, de plus, une certaine tendance à se recourber, enfin, s'atténuant en un rostre obtus, un tant soit peu troncatulé, inférieur et regardant en bas.

Sommets convexes, très obtus, non proéminents, toujours fortement excoriés, et, lorsqu'ils le sont un peu moins, laissant voir les traces de rides robustes et espacées. Arête dorsale arquée comme le contour du bord supérieur, de plus bien convexe (convexité s'atténuant brusquement sur la crête qui est nulle et atrophiée).

Stries grossières, rugueuses, çì et lì saillantes, enfin feuilletées vers les contours. Epiderme terne d'un marron noirâtre presque uniforme, encrassé sur todte la région postérieure et fortement rongé vers les sommets. Intérieur d'une nacre peu brillante d'une nuance blanche-bleuacée, avec de larges taches livides-jaunacées.

Ligaments: antéro-interne fort, épais, se poursuivant 
sous l'autre ligament jusquàa 17 mill. en arrière des crochets pour se terminer par une lunule interne; postérieur robuste et saillant. Lunule courte, triangulaire. Charnière ondulée avec plusieurs saillies et deux ou trois dépressions. Impressions profondes, irrégulières.

Cette Anodonte vit en Lombardie, d'où elle m'a été adressée sous l'appellation fautive d'idrina, sans indication précise de localité. D’après l'aspect de son épiderme, la Carote doit habiter des eaux courantes à fond de graviers, probablement la décharge d'un de ces lacs si nombreux dans cette partie de l'Italie.

\section{Anodonta Bunotain.}

Espèce très caractérisée par sa forte direction descendante. Le point central du contour rostral forme un angle de $60^{\circ}$ avec le plan de la ligne des sommets et un de $30^{\circ}$ avec la verticale de la perpendiculaire.

Long. $\max . \ldots \ldots \ldots \ldots \ldots \ldots \ldots$

68 mill.

Haut. max......................
Épaiss. $\max$. (d 19 des sommets et de l'angle postéro-dorsal; à 41 du rostre, à 31 du bord antér., à 28 de la perpendiculaire. $). . . . . . . . . . . . . .$.

Long. de la crête ligamento-dorsale, des sommets à l'angle postéro-dorsal.....

Dist. de cet angle au rostre...........

Corde apico-rostrale............. 60 -

Haut. de la perpendiculaire.......... 42 -

Dist. de la perpend. au bord antér... 21 - 
Dist. du même point de cette perpend. au rostre................. 47 mill.

- enfin, de la base de la perpendiculaire à l’angle postéro-dorsal.... $40 \quad-$

Chez cette Anodonte, la convexité, qui est très régulière, est rapprochée des sommets et de l'angle postérodorsal; la crête n'est pas atrophiée comme chez la précédente; l'angle est accusé; les sommets, non convexes, sont comme écrasés; le rostre regarde tout à fait en bas, par suite de la grande inclinaison descendante de la région postérieure.

Coq. à test lisse, moins rugueux, plus brillant et à valves un peu plus fortement bâillantes que celles de la Carota.

Bord supérieur arqué jusqu'à l'angle, puis descendant à pic sur le rostre d'une façon rectiligne. Région antérieure arrondie, excessivement décurrente à la base. Bord inférieur très descendant, tout en étant très faiblement arqué. Région postérieure très inclinée-descendante, un peu plus de deux fois plus longue que l'antérieure, augmentant plus en hauteur, et, s'atténuant en un rostre subarrondi tout à fait inférieur et regardant en bas.

Sommets aplatis, tournés en avant, sillonnés par de petites rides peu prononcées. Crochets aigus. Arête dorsale confondue dans la convexité. Crête à peine accusće, faiblement comprimée vers l'angle, qui est obtus, bien qu'assez saillant.

Stries généralement fines et régulières, plus fortes seulement ver's les contours. Épiderme peu brillant, d'une teinte uniforme d'un brun-oliveûtre, s'éclaircissant vers les sommets en une nuance jaune-rougeâtre. Intérieur d'une nacre bleuacée peu irisée. 
Ligaments: antéro-interne épais, se poursuivant sous l'autre ligament, jusqu'à 1 ว̆ mill. en arrière des crochets et terminé également par une lunule; postérieur robuste, saillant. Lunule triangulaire, charnière peu ondulée, avec une légère éminence lamelliforme à la cardinale.

J'ai recueilli cette Anodonte dans un petit cours d'eau aux alentours de Troyes (dube). Elle vit également dans le lac des Quatre-Cantons, près de Kussnacht, en Suisse.

\section{Anodonta abbreviata.}

Anodonta anatina typica, Brot, nayades du Léman, pl.V. f. 2 , et Anondonta anatina, var. abbreviata, Brot (même ouvrage), pl. VI, f. 2, 1867.

Cet Acéphale, très bien figuré par Brot, „a été recueilli dans le lac de Genève, près de Pully, aux environs de Vevey, enfin, aux Pâquis dans une anse un peu abritée des vagues, présentant un fond peu vaseux mélangé de cailloux. - Je le connais encore du lac d'Annecy, en Savoie.

\section{Anodonta illuviosa.}

Anodonta anatina monstrosa, Brot, nayades du Léman, pl. VII, f. 2, 1867.

Lac de Genève, près de la ville du même nom, aux Pâquis, à l'entrée du fossé vert (Brot).

Je possède des ćchantillons complètement identiques à ceux de Genève, du lac du Quatre-Cantons, entre Lucerne et Kussnacht. J'en ai reçu également de semblables du 
ruisseau de la Salle, dans Saône-et-Loire (Locard), et de l'Yvette à Orsay, près Paris (Coutagne).

\section{Anodonta fallax.}

Anodonta fallax, Colbeau, moll. Belg. in : ann. soc. Belg. III, p. 107, pl. III, f. 3, 1868.

Cette espèce a encore été figurée:

$1^{\circ}$ Sous le num de Mytilus anatinus var. fig. 4., par Maton et Rackett (Desc. cat. Britr test. in: Linn. trans. VIII, 1807, - et (traduction Chenu) pl. XVI, f. 4, 1845), d'Angleterre, sans indication de localité;

$2^{\circ}$ Sous l'appellation d'A nodonta oviformis, par Clessin (Anod. in: $2^{e}$ édit. Chemnitz, p. 88, pl. XXVI, f. 5, 1876); du lac de Constance, en Suisse.

La fallax vit dans les cours d'eau d'Angleterre, de Belgique (Colbeau n'indique pas de nom de localité), et, dans les lacs de la Suisse (tels que le lac de Constance (Clessin) et celui des Quatre-Cantons, où je l'ai recueillie entre Lucerne et Kussnacht.

Je ne la connais pas de France.

\section{Anodonta pssmmita.}

Anodonta psammita, Bourguignat, Malac. Quatre-Cantons, p. $\ddot{8} 8, \mathrm{pl}$. IV, f. 1-3, 1862 .

Jolie espèce que j’ai trouvée dans le lac des Quatre-Cantons, près de Bergischwyl.

Je n'ai pas signalé les signes distinctifs des Racketti, 
abbreviata, illuviosa fallax et psammita, parce que le figures que j'ai citées pour chacune de ces espèces sont excellentes et rendent bien l'aspect et les caractères de ces Acéphales. J'ajouterai, en outre, que s'il me fallait décrire toutes les Anodontes que je mentionne, je ne finirais point.

C'est dans ce groupe, à mon sens, que devrait prendre place la vraie leprosa (Parreyss) du Piémont, qui m'est imparfaitement connue, espèce, dont les auteurs ont dénaturé les caractères en voulant la faire entrer dans la série des Idrina.

\section{PSO BRIANDIANA}

Les Anodontes de ce groupe, loin d'être oblongues comme les Abbreviatiana, sont des espèces subarrondies ou subtrigonales excessivement hautes, par rapport à leur longueur, caractérisées, en outre, par une région postérieure relativement très courte, dont l'extrémité, à partir de l'angle postéro-dorsal jusqu'au rostre, semble comme tronquée, sauf chez la callosa, par suite de la direction presque aplomb du contour, La crête dorsale n'est pas atrophiée, comme chez les Abbreviatiana.

Les Briandiana habitent, comme les précédentes, les cours d'e: u à fond de graviers, ou, les lacs dans les endroits sableux non vaseux.

\section{Anodonta Callosa.}

Anodonta Callosa, Held, in: Kïster, Anod. in : $2^{\circ}$ édit. Chemnitz, p. 36, pl. IX, f. 1, 1852 .

La callosa, espèce très caractérisée, est bien représentée 
par Küster. Elle a été recueillie dans la haute Bavière, dans le lac de Chiem. Notre ami le docteur Servain l'a trouvée assez abondamment sur les bords du lac Balaton, en Hongrie.

Je l'ai reçue de Mörch, sous l'appellation fautive d'Anodonta macula, var. 1, de Viborgsö (Danemark).

\section{Anodonta Priandiana.}

Anodonta Briandiana, Servain, Malac. Balaton (sous presse), 1881.

Cette belle espèce, que le $\mathrm{D}^{\mathrm{r}}$ Servain a recueilli au-dessous de Siö-Fok, sur les bords du lac Balaton, en Hongrie, est remarquable par sa grande hauteur (61 mill.), comparée à sa plus grande longueur ( 80 mill.). Ses valves bâillantes en avant et en arrière, mais surtout en avant, ont une forme absolument subtriangulaire; sa crète est comprimée et son ligament entièrement recouver t.

Chez la Briandiana, la région postérieure n'atteint pas deux fois le développement de l'antérieure; son contour, entre l'angle postéro-dorsal et le rostre, descend presque à pic sur une partie rostrale inférieure excessivement obtuse.

\section{Amodonta Fenoun.}

Anodonta Renoufi, Servain, Malac. Balaton (sous presse), 1881.

La Renoufi, également récoltée sur les bords du lac Balaton, est une forme moins allongée (72 mill.) et relati- 
vement plus haute ( 37 mill.) que la précédente. Elle offre un contour subtrigonal, dont les trois angles sont encore plus obtus que ceux de la Briandiana, notamment l'angle postéro-dorsal. Si la région antérieure n'était pas décurrente à sa base, celte espèce pourrait presque se rapprocher de la forme circulaire. Chez elle, la convexité maximum est à égale distance du bord antérieur et du rostre. Les valves, assez épaisses (celles de la précédente sont minces), d'une teinte uniforme d'un corné-marron excessivement claire sur les régions ventrale et ombonale, n'ofirent qu'un seul bâillement antérieur. Le ligament, comme chez la Briandiana, est recouvert.

\section{Anodonta Truncata.}

Anodonta Truncata, Parreyss, mss. in : Brusina, Contrib. moll. Dalm., p. 131 (sans descript.), 1866.

Espèce à valves non bâillantes, à peu près de même forme et de mêtue contour que celles des Briandiana et Renoufi, mais d'une taille bien inférieure. Chez quelques échantillons la région postérieure paraît malade et la troncature semble comme une conséquence de cet état maladif. Dans ce cas, la convexité maximum est très rapprochée du bord postérieur. Mais, chez les individus nor. maux, le point maximum est presque central.

Long. $\max . . \ldots \ldots \ldots \ldots \ldots \ldots . . . . . .53$ mill.

Haut. $\max . \ldots \ldots \ldots \ldots \ldots \ldots \ldots, \quad 40$.

Epaiss. max. (i $18 \mathrm{l} / 2$ des sommets, de l'angle postéro-dorsal et de la base de 
Ia perpend., à $28 \mathrm{du}$ rostre, à $26 \mathrm{du}$ bord antérieur)................

Long. de la crête ligam.-dorsale, des sommets à l'angle postéro-dorsal........

Distance de cet angle au rostre........ $32 \quad-$

Corde apico-rostrale.............. 46 -

Haut. de la perpendiculaire.......... 330

Dist. de cette perpend. au bord antér... 20 -

- du même point de cette perpend. au rostre.....................

- enfin, de la base de la perpend. à l'angle postéro-dorsal.............

Bord supérieur très faiblement arqué jusqu'à l'extrémité du ligament, puis formant, à partir de ce point, une courbe tombant presque à pic sur le rostre. Région antérieure arrondie, décurrente à la base. Bord inférieur légèrement rectiligne et assez descendan jusqu'à son extrémité. Région postérieure fort ćcourtée, un peu plus d'une fois et demie plus longue que l'antéricure, augmentant en hauteur de 5 mill. (par suite de la direction descendante du bord inférjeur) jusqu'au niveau de l'extrémité du ligament, puis à partir de là, se tronquant presque subitement et se terminant par une partie rostrale exiguë, tout à fait inférieure.

Sommets convexes, non saillants ni ventrus, sillonnés par des rides élégantes. Crochets très aigus. Arête dorsale ordinairement confondue dans la convexité, d'autre fois excessivement tuméfiée. Crête médiocre (quelquefois nulle), à peine comprimće.

Stries grossières, çà et là plus ou moins saillantes, devenant sur toute la rígion postérieure, des sommets au 
rostre, rugrueuses et comme imbriquées. Épiderme assez terne, d'une teinte uniforme tantôt d'un brun olivacé avec des zones alternatives plus claires et plus foncées, tantôt d'un ton cendré-violacé passant vers le bord palléal à une nuance opaline. Intérieur àune nacre bleuacée peu irisée.

Ligaments : antéro-interne filiforme; postérieur court, robuste et proéminent. Lunule allongée.

La Truncata vit en Dalmatie, dans la Kerka près de Visovac, où elle est abondante.

\section{Anodonta Colloba.}

Petite Anodonte comprimée, à valves minces, à peine bâillantes en avant et en arrière, possédant à peu près les mêmes contours que les précédentes, seulement dans des proportions plus réduites, par suite de sa petite taille.

Long. $\max . . . \ldots \ldots \ldots \ldots \ldots . . . . . . .68$ mill.

Haut. $\max . . . . . . . . . . . . . . . . .635$ -

Epaiss. max. (à 16 du sommet, à 24 du rostre, à 26 du bord antérieur, à 14 de l'angle postéro-rostral, à 19 de la base de la perpend.)................

Lung. de la crête ligam.-dors., des sommets à l'angle postéro-dorsal.......... 23 -

Dist. de cet angle au rostre.......... 24 -

Corde apico-rostrale.............. $\$ 1$ -

Haut. de la perpendiculaire......... 32 -

Dist. de celte perpend, au bord antér... 17 -

- du même point de cette perpend.au rostre..................... 
Dist. enfin, de la base de la perpend. à

l'angle postéro-dorsal....... 30 mill.

Bord supérieur arqué jusqu'à l'extrémité du lignament, puis éprouvant à l'endroit de la lunule une dépression très prononcée; enfin, à partir de l'angle, descendant sur le rostre d'une façon presque rectiligne. Région antérieure arrondie, décurrente à la base. Bord inférieur rectiligne-descendant. Région postérieure un peu plus d'une fois et demie plus longue que l'antérieure, augmentant en hauteur jusqu'au niveau du milieu (et non de l'extrémité) du ligament, puis s'atténuant brusquement, pour se terminer par un rostre inférieur arrondi très obtus.

Sommets aplatis, comme écrasés, non proéminents, toujours excoriés. Arête presque nulle. Crête très médiocre, avec l'angle postéro-dorsal très émoussé.

Stries grossières, çà et là rugueuses, imbriquées vers le bord postérieur. Épiderme recnuvert d'un enduit noir foncé, très tenace, et, lorsqu'il est enlevé, offrant une teinte uniforme d'un ton olivâtre. Intérieur d'une nacre bleuâtre avec de larges taches jaunacées livides.

Ligaments : antéro-interne filiforme; postérieur court, saillant, aux trois quarts recouvert. Lunule allongée, subtriangulaire. Charnière avec une saillie lamelliforme en dessous des crochets.

La colloba, habite dans la Canne près de Saint-Saulge (Nièvre), d'où je l'ai reçue sous le nom d'anatina.

\section{Ho AVONIANA}

Ces Acéphales sont comprimés, d'une forme obtusément tantôt subquadrangulaire (incrassata, Avonica), tantôt 
subtrigonale (Dosori). Les sommets sont cumme écrasés et aplatis; enfin, leurs valves sont pesantes et épaisses, comme celles des Ponderosiana.

Les Avoniana vivent dans les eaux peu courantes, limpides, à fond généralement vaseux.

\section{Anodonta incrassata.}

Mytilus incrassatus, Sheppard, on two new Brit. spec. of Mytilus, in : Linn. trans. XIII, p. \&ö, pl, V, f. 4, 1820, et (traduct. Chenu), p. 270, pl. XXVII, f. 2, 1895.

Cette espèce, que tous les auteurs ont confondue bien à torl avec la ponderosa, dont elle est tnut à fait dissemblable, est Irìs bicn représentée dans le travail de Sheppard, ainsi que sur les planches de la Iraduction du $\mathrm{D}^{\mathrm{r}}$ Chenu.

Elle vit dans la rivière de Trent, à Holme près de Newark, dans le comté de Nottingham en Angleterre. Je. ne la connais pas ailleurs.

\section{Anodonta sonica.}

Mylilus Aronensis, Montagu, Test. Brit., p.172, 1803.

Cette Anodonte a été parfaitement figurée sous le nom d'Anodon Avonensis, var. 7, par Th. Brown, dans ses illustrations conchỵliologiques de la Grande-Bretagne (pl. XVIII, f. 3, 1845). Je renvoie à cette figure pour la connaissance de cette espèce.

Ioptagu signale l'Avonensis (mieux Avonica, puisque l'Avon est une rivière) de l'Avon près Lackham dans le Wiltshire. Brown la mentionne encore près de Tisbury, à 
Burton sur le Trent, à Steelon, à Halifax dans le canal, enfin, près de Sowerby-Bridge dans le Yorkshire.

En France, je la connais typique du Morin à Esbly dans Seine-at-Marne (Mabille), et, du canal de Loir-et-Cher (Rambur).

\section{Anodonta Destiri.}

Anodonta Desori, Coutagne, in litt.

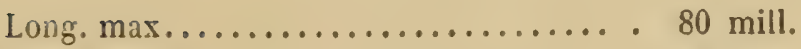

Haut. $\max . . . \ldots \ldots \ldots \ldots \ldots \ldots . . . . .658$ -

Épais. max. (à 22 des sommets, à 50 du rostre, à 32 du bord antér., à 30 de l'angle postéro-dorsal, à 28 de la base de la perpend.)..................... 20 -

Long. de la crête ligam.-dors., des sommets à l'angle postéro-dorsal............ 37 -

Distance de cet angle au rostre......... 44 -

Corde apico-rostrale............... 70 -

Haut. de la perpendiculaire........... 48 -

Dist. de celle perpend. au bord antér.... 24 -

- du même point de ce!te perpend. au rostre..................... 56 -

- enfin, de la base de la perpend. à l'angle postéro-dorsal............. 54 -

Coq. de forme obtusement subtrigone, relativement très aplatie ( 20 mill.) pour sa taille, puisque sa convexité n'est que le quart de la longueur, à válves épaisses, solides médiocrement bâillantes en avant et en arrière.

Bord supérieur rectiligne jusqu'à l'extrémité du ligaMAI 1881. 
ment, puis d'abord faiblement incliné jusqu'à l'anorle, enfin, descendant presque à pic et en ligne droite jusqu'au rostre. Région antérieure arrondie, décurrente à la base. Bord inférieur convexe-descendant s'arrondissant à son extrémité. Région postérieurs un peu plus de deux fois plus longue que l'antérieure, allant en augmentant fortement (de 10 mill.) jusqu'au niveau de l'extrémité du ligament, par suite du développement du bord inférieur, ensuile allant en s'atténuant brusquement, pour se terminer inférieurement par une partie rostrale infúrieure arrondie très obtuse.

Sommets aplatis, écrasés, non convexes, sillonnés de rides concentriques tremblotées. Crochets aigus. Arête dorsale non saillante ni convexe, confondue dans la convexité, néanmoins limitée supérieurement par un léger sillon descendant obliquement des sommets à la partie supérieure du rostre, qu'il limite. Crête développée, comprimée.

Stries fortes, irrégulières, çà et là plus saillantes, un peu feuilletées et imbriquées vers les contours. Épilerme brillant, d'un ton gris-olivacé en avant et d'une nuance rouge-brique en arrière. Intérieur d'une nacre peu irisée d'un blanc-bletracé.

Ligaments : antéro.interne filiforme; postérieur fort robuste, proéminent, terminé par une longue lunule.

Cette Anodonte dédiée au savant paléontologriste Desor, vit dans le lac de Neuchâtel, près de la ville du même nom, en Suisse (Goutagne). 


\section{SPENGLEIRANA}

Les Acéphales de ce groupe, bien que formant une série naturelle, sont des Anodontes difficiles à bien caractériser, parce qu'elles unt certaines apparences de piscinales sans en avoir les vrais signes distinctifs. Chez ces Anodontes, toutes d'une coloration sombre (sauf la tricassina), les valves sont relativement assez épaisses; l'arête dorsale, dans la plupart des espèces, très bombée, s'étend dans une direction rectiligne, parfois un peu arquée, en tous cas, jamais concave comme chez les véritables piscinales ; la région postérieure, médiocrement allongée, s'atténue en un rostre très obtus, non prolongé et ne présentant jamais une tendance ascendante; le contour postérieur, entre l'angle postéro-dorsal et le rostre, à l'exception de celui de la minima, descend d'une façon brusque sur la partie rostrale, etc. - Les figures de la trasymenica (iconogr. fig. 1958) et de l'intermelia (Encycl. méth., pl. 201, f. 2), donnent une idée exacte de la forme et de l'aspect des espèces de ce groupe.

Les Spengleriana vivent dans les eaux claires, à fond vaseux, des lacs et des rivières.

\section{Mnodonta trasymenica.}

Cette Anodonte, primitivement prise pour une piscinalis par quelques malacologistes italiens, a été bien représentée (fig. 1958) sous l'appellation d'Anodonta idrina, var. trasymenica (Iconogr. VII, band, p. 33̈) par l'auteur des suites à Rossmässler. 
Celte espèce paraît ábondante dans le lac de Pérouse, en Italie.

\section{Anodonta meretrix.}

La meretrix vit avec la précédente, arec laquelle elle a été confondue sous les noms erronés de piscinalis ou d'idrina.

Elle differe de la trasymenica par sa taille constamment moindre; par sa région postéricure plus écourtée, dont la partie rostrale (qui est inférieure) regarde en bas: par son contour, de l'angle postéro-dorsal au rostre, descendant plus rapidement; par son bord inférieur sinué à sa partie médiane; par sa région antérieure relativement plus grande et plus développée, puisqqu'elle atteint 37 mill., comme celle de la trasymenica; par sa convexité plus rapprochće du rostre (à 36 seulement, tandis que celle de la trasymenica est à 44 mill.); par son arête dorsale plus hombée, offrant une descente plus rapide sur la crête, qui est moins comprimée; par ses sommels plus médians, etc...

Voici la mensuration comparative des deux espèces :

$$
\begin{array}{cc}
\text { mere- } & \text { trasy- } \\
\text { trix. } & \text { menica }
\end{array}
$$

Long. max............. 92 mill. 100 mill.

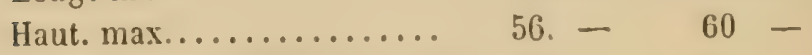

Epaiss. max. (à 36 des sommets, et du rostre, à 530 du bord antérieur, à 26 de l'angle postéro-dorsal, à 27 de la base de la perpend. Épaiss. max. de la trasymenica (à $\mathbf{3} 7$ des sommets, à $\mathbf{4} 4$ du

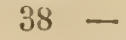


rostre, $\grave{3} 56 \mathrm{du}$ bord ant., à 30 1/2 de l'angle postérodorsal, et de la base de la perpendiculaire).......... 40 mill.

Long. de la crête ligamento. dorsale, des sommets à l'an. gle postéro-dorsal........ Dist. de cet angle au rostre. Corde apico-rostrule...... Haut. de la perpendiculaire. Dist. de cette perpend. au bord antér.............

- du même point de la perp . au rostre........

- enfin, de la base de la perpend. à l'angle postérodorsal.............

On remarquera: $1^{\circ}$ que la région antérieure des deux espèces ont la même mensuration, tandis que la région pustérieure de la meretrix a 10 mill. de moins que celle de la transymenica; $2^{\circ}$ que la convexité maximum de la meretrix, à égale distance des sommets et du rostre, est bien plus rapprochée de l'extrémité rostrale que du bord antérieur, lorsque chez la trasymenica, ce mẻme maximum est moins distant des sommets que du rostre. Ces différences, dans la longueur de la région postérieure, dans la position dilférente du maximum de convexilé, donuent à ces espèces une apparence très dissemblable l'une de l'autre.

Coq. brillante, ordinairement d'une teinte sombre, à valves présentant deux bâillements très peu ouverts (ceux de la trasymenica sont plus dilatés). 
Bord supérieur rectiligne jusqu'à l'angle postéro-dorsal, puis descendant presque brusquement en ligne droite sur le rostre. Région antérieure largement développée, arrondie-oblongue. Bord inférieur convexe, légèrement sinuéconcave vers la partie moyenne. Région postérieure écourtée dans une direction obliquement descendante (celle de la trasymenica est oblongue dans une direction plus horizontale), une fois et demie plus longue que l'antérieure, paraissant comme tronquée à partir de l'angle postéro-dorsal, et se terminant par un rostre obtus, arrondi (non troncatulé), et de plus presque tout à fait inférieur.

Sommets peu convexes, médiocrement ventrus, sillonnés de rides concentriques, robustes, légèrement ondulées. Grochets très aigus, ordinairement rongés. Arête dorsale très bombée, s'accusant par deux radiations brunes, divergentes (dont la supérieure un tant soit peu saillante), et, ofirant, en outre, supérieurement une direction descendante très rapide sur une crête dorsale médiocre et peu comprimée.

Stries fines, devenant de plus en plus saillantes vers les contours, où elles se montrent souvent rugueuses et feuilletées. Épiderme très brillant, d'une teinte noir-olivâtre, s'éclaircissant vers les sommets, avec une multitude de petites radiations indécises d'un ton plus foncé. Quelquefois l'épiderme est d'un ton jaune-verdâtre (comme celui de la trasymenica). Cette variété parait rare. Intérieur d'une belle nacre irisée de nuance bleuacée.

Ligaments : antéro-interne médiocre, très antérieur; posterieur peu saillant, assez court et presque entièrement recouvert. Lunule petite, triangulaire. 


\section{Anodonta intermedia}

Anodonta intermedia, Lamarck, Anim. s. vert. VI, $1^{\text {er }}$ part., 1819, p. 86.

Personne jusqu'à présent n'a su comprendre l'intermedia de Lamarck, et celı, parce que sous ce norn, Lamarck a réuni deux formes essentiellement différenles.

Je cite la description de Lamarck.

«AN. INTERMEDIA : testa ovata, subradiata, postice ${ }^{1}$ brevi rotundata; pube elevata, compresso-carinata; natibus retusis. - Schroter, flussconch. pl. 1, t. 2, et Encycl. méth., pl. 201, f. 2. Habite en France dans la Loire. Cabinet de M. Dufresne. - VAR, B. testa minore; radiis nullis. Schroter, flussconch., pl.1, f. 3. La variété B. se trouve dans la Seine (mon cabinet)., etc... $D$

Il y a donc une intermedia type de la Loire que Lamarck n'a jamais possédé, qui appartenait à un M. Dufresne. Cet échantillon, qui a passé en Angleterre depuis fort longtemps, avec la collection Dufresne, se trouve actellement je ne sais où.

Cette inlermedia TYPE, que personne ne connaît, est caractérisée, dit Lamarck, par une « pube elevata, compressocarinata, « c'est-à-dire par ce que je désigne maintenant par une crête dorsale élevée, comprimée, par cela même carénée (ce caractère ne convient nullement à l'oblonga ni à la cygncea); le test est a ovata » et " subradiata » (autre caractère qui ne correspoud pas avec la forme oblongueallongée des espèces de tout le groupe des cygnœa). A ces

1. Pro antice. 
caractères bien tranchẻs, nettement exprimés, Lamarck ajoute que son intermedia ressemble aux finures de la pl.201 (fig. 9) de l'Encyclopédie méthadique et de pl. I (fig. 2) des Flussconchylien de Schroter. Or, lorsqu'ou examine ces figures, on voit qu'elles sont parfaitement identiques l'une à l'autre; de plus, qu'elles sont très bien exécutées. D'après ces figures, dont les contours et l'aspect $\mathrm{c}$. drent bien avec les signej distinctifs assignés par Lamarck à son espèce, on reconnaît une Anodonte du groupe des Spengleriana, groupe ayant déjà une certaine tendance à se rapprocher de celui des Piscinales.

La seconde espèce, désignée var. B. lesta minore, vadiis nullis, trouvée dans la Seine (j'ai su qu'elle provenait d'un vieux bras de la Scine à Javel, aujourd'hui comblé), est représentée dans Schroter à la figure 3 de la planche pre. mière de ses Flussconchylien. Cette figure, très bien faite, donne l'aspect d'une petite Anodonte voisine de la minima de Nillet, espèce appartenant, à mon sens, au groupe des Spengleriana, au même titre que l'intermedia. Lamarck nes'élait done pas trompé sur l'ensemble des caractères des deux formes qu'il avaient réunies sous la même appellation.

Or, au sujet de cette variété (que les auteurs de nos jours ont confondue, sans réflexion, avec le type intermedia de la Loire), il s'est élevé un débat.

Dans la collection de Lamarck ${ }^{1}$, Joannis, qui tenait beaucoup à savoir ce que pouvait être l'intermedia, a reconnu (Etud. Naïod. in : Ann. soc. linn. Maine-etLoire 1859, p. 272) :

1. Gette collection, appartenant jadis à M. Delessert, se trouvo actuellement à Genève. 
$1^{\circ}$ Que le type de la Loire n'existait pas;

$2^{\circ}$ Que le type de la variété B. de la Seine, collé sur un carton portant encore le $n^{\circ}$ d'ordre de la main de Lamarck, était une véritable oblonga de Millet. " On se demande, ajoute Joannis, comment l'exemplaire du cabinet Lamarck a pu ressembler à la figure qui est dans l'Encyclopédie ', si différente pour la forme de l'exemplaire du cabinet de Lamarck, aujourd'hui chez M. Delessert. A cela, il y a une réponse qui accordera tout le monde. C'est qu'à l'époque où Lamarck à fait son travail sur les mollusques, il était devenu presque complètement aveugle et ne jugeait guè: $\mathrm{E}$ les formes que par le toucher, il est donc probable qu'il y a eu quelques erreurs commises par Lamarck luimême. »)

Eh bien, non! s'il y a eu erreur, elle n'a pas été commise par Lamarck, mais par le $D^{r}$ Chenu, que j'ai vu, de mes yeux vu, en 1852 et 1853 , dans le but de nettoyer les échantillons de Lamarck, les décoller tous pour les passer à l'eau; en la recollant, ce brave docteur se sera trompé de carton, de là l'erreur attribuée à Lamarck et relevée par Joannis.

A l'époque où ce savant professeur a composé le texte de ses Anodontes (j'ai su par des notes conservées au muséum, que c'était en 1817), cet illustre zoologiste n'avait pas encore perdu la vue. Ce qui le prouve, c'est LE N ${ }^{\circ} D^{\prime}$ ORDRE DE LA MaIN de Lamarck, $\mathrm{N}^{0}$ vU par Joannis. Il voyait donc, lorsqu'il a nommé celte espèce. Il ne perdit la vue qu'en 1819, au moment ou il publiait les dernières feuilles de la première partie du tome VI de ses « Animaux sans

1. Je ferai remarquer que Lamarck n'a ' jamais dit que sa var. B ressemblait à une figure quelconque de l'Encyclopédie. 
vertèbres ". Lamarck, lui-même, nous l'apprend dans son introduction. « A la suite d'une ophtalmie desplus violentes, des cataractes, s'étant manifestées sur mes yeux, parvinrentà me réduire à une cécité complète. J'étais alors à peu près au milieu du $\mathrm{VL}^{e}$ volume de cet ouvrage », et plus loin il avoue que dans ce volume il n'y a que les espèces de deux genres, dont il n'a pas pu vérifier, de ses propres yeux, les caractères et les synonymies, « les Oscabrions et les Patelles, dit-il, ont eu à supporter l'imperfection de mes moyens. ")

Lamarck n'a donc pu comettre cette bévue dont l'accuse Joannis. Le $\mathrm{D}^{\mathrm{r}}$ Chenu seul en est capable.

D'un autre côté, M. Drouët prétend avoir vu dans la collection de mon regretté ami Deshayes une Anodonta oblonga étiquetée intermedia; de là, sans vérifier ni étudier le texte des Animaux sans vertèbres, il affirme subitement que l'espèce de Lamarck est une oblonga. Eh bien! en admettant qu'une oblonga ait été étiquetée intermedia dans la collection de Deshayes, qu'est-ce que cela prouve ! cela prouve simplement qu'il y avait des espèces (ou du moins une) mal nommées dans cette collection, et, pas autre chose.

Lorsquon parcourt les ouvrages malacologiques, on voit surgir, à ce sujet, un assez grand nombre d'opinions diverses.

Moquin-Tandon (Moll. France, II, p. 55็7), qui a subi pour les Naïades, l'influence de M. Drouët, regarde l'intermedia comme une oblonga.

L'abbé Dupuy (Moll. France, p. 603 et 604) la rapporte à $s a$ cellensis.

Rossmässler (lconogr.1, 1835, p. 111), présume que cette espèce est une Cygncea non adulte. 
Brown (illust. pl. XVII, f. 1, 2), représente, comme elle, une forme voisine de ma Sturmi, du groupe des Depressiana.

Clessin et Küster restent muets sur cette espèce.

Quant à C. Pfeiffer, on trouve dans son travail deux intermedia différentes :

$1^{\circ}$ (Naturg. Moll. 1, 1821, p. 113, pl. VI, f. 3), une forme analogue à celle de l'ouvrage de Brown, voisine de ma Sturmi, du groupe des Depressiana.

$2^{\circ}$ Et $(11,1825$, pl. V.) une autre forme oblongue (qui m'est inconnue), que je crois voisine, bien que différente, de la fragillima de Clessin, du groupe des Ventricosiana, etc., etc.

En résumé :

Deux espèces d'intermedia dans Lamarck, une première de la Loire (c'est celle-là qui est le type), bien caractérisée et parfaitement figrurée dans les planches de l'Encyclopédie et de Schröter; puis, une seconde de la Seine, bien plus petite, voisine de la minima et très dissemblable de celle de la Loire.

J'ajouterai, enfin, qne ces deux espèces n'ont aucuns rapports avec les soi-disants intermedia des auteurs, qui ne les ont pas connues, parce qu'ils n'ont pas pris la peine d'étudier le texte des Animaux sans vertèbres.

Je renvoie pour la connaissance de la vRaIE intermedia, aux figures citées par le savant Lamarck. Elles sont excellentes et elles rendent bien l'aspect et les caractères de cette Anodonte.

Je possède des échantillons complètement semblables au type de Lamarsk. Ils ont été recueillis dans la Loire, à Nantes et à Tours, par MII. Rambur et Mabille. J'ai reçu également cette espèce des environs de Manchester, en 


\section{$-316-$}

Angleterre, où elle a été pêchée dans l'Irwell, ainsi que des rivières autour d'Andrinople, en Rumélie. Je sais, enfin, qu'elle vit dans les cours d'eau de la Bavière.

\section{Anodonta sedentaria.}

Anodonta sedentaria, Mabille, in litt.

Cette espèce est remarquable parsa convexité maximum fort peu distanie des sommets. Cette convexité est fort régulière, bien que très portée sur la région ombonale.

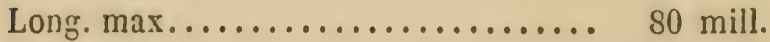

Haut. $\max . . . \ldots \ldots \ldots \ldots \ldots$. $471 / 2$

Epaiss. max. (à 13 des sommets, à 48 du rostre, à 34 du bord antér., à 27 de l'angle postéro-dorsal, à 34 de la base de la perpend.).................

Long. de la crête ligam.-dors., des sommets à l'angle postéro-dorsal ....... Dist. de cet angle au rostre...........

Corde apico-rostrale............ 60 -

Haut. de la perpendiculaire......... 46 -

Dist. de la perpend, au bord antér.....

- du même point de la perpend. au rostre ....................

- enfin, de la base de la perpend. à l’angle postéro-dorsal........ 50 -

Bord supérieur rectiligne jusqu'à l'angle postéro-dorsal, puis offrant un contour légèrement concave en descendant sur le rostre. Rígion antérieure grande, arrondie, un 
tant soit peu décurrente ver ja la base. Bord inférieur exactement convexe dans toute son étendue. Région postérieure près de deux fois plus longue (moins 3 mill.) que l'antérieure, augmentant fort peu en hauteur jusqu'au niveau d'une verticale tombant du milieu du ligament postérieur, et, allant ensuite, en s'atténuant en une partie rostrale arrondie, non descendante, ni ascendante, mais horizontale.

Sommets convexes, bien que non proéminents, sillonnés par des rides élégantes. Arète dorsale confondue dans la convexité, s'accusant sculement par une radiation plus foncée, qui souvent fait défaut. Crête peu dilatée, comprimée.

Stries assez fortes, egà et là plus saillantes, surtout très feuilletées vers la rẻgion des contours. Épiderme assez terne, d'une teinte sombre-noirâtre, avec quelques zones d'une nuance olivâtre foncée; enfin, passant sur la région des sommets à un ton rouge-brique. Intérieur d'une nacre d'un blanc-mat.

Ligaments : antéro interne très antérieur, robuste; postérieur saillant, peu allongé et à moitié recouvart. Lunule trìs grande (11 mill.).

La sedentaria vit dans la Loire et dans l'Er」re, plès de Nantes (IIabille), ainsi que dans la Masle près d'Amboise (Rambur).

Je l'ai reçue de Mörch, sous l'appellation d'inornata, variété, comme provenant d'Hofmansqrave, en Danemark.

\section{Anodouta spengleri.}

Cette Anodonte, plus oblongue et moins haute que la précédente, a une convexité maximum presque centrale. 
La longueur de la crête, des sommets à l'angle postérodorsal, est égale à celle de cet angle au rostre, ce qui se remarque rarement. Ordinairement la distance de l'angle au rostre dépasse toujours celle de l'angle aux sommets, sauf chez une certaine série des Cygnoeana, comme chez les Nansoutyana, Moulinsiana, etc., où la distance des sommets à l'angle l'emporte sur celle de l'angle au rostre.

Les valves de la Spengleri, assez épaisses, sont à peine bâillantes.

Long. $\max . \ldots \ldots \ldots \ldots \ldots \ldots \ldots$........ 8 mill.

Haut. $\max . . . \ldots \ldots \ldots \ldots \ldots . . . .46$ -

Épaiss. max. (à 23 des sommets, à 45 du rostre, à $40 \mathrm{du}$ bord antér., à $27 \mathrm{de}$ l'angle postéro-dorsal, à 25 de la base de la perpend.).................

Long. de la crête ligamento-dorsale, des sommets à l'angle postéro-dorsal..... 35 -

Dist. de cet angle au rostre.......... 35 -

Corde apico-rostrale.............. 64 -

Haut. de la perpendiculaire.......... 42 -

Dist. de cette perpend. au bord antérieur. 27 -

- du même point de cette perpend. au rostre.................. 58 -

— enfin, de la base de la perpend. à l'angle postéro-dorsal.............. 50 -

Bord supérieur rectiligne jusqu'ì l'angle, puis descendant en ligne droite sur le rostre d'une façon moins inclinée que celui de la sedentaria. Région antérieure bien arrondie, moins développée en hauteur que celle de la précédente. Bord inférieur convexe (mais à un degré 
moindre que celui de la sedentaria) dans toute son étendue, avec un sentiment de convexité un peu plus prononcé vers son extrémité. Région postérieure allongée, un peu plus de deux fois plus longue que l'antérieure, augmentant en hauteur de 4 millimètres, au niveau d'une verticale tombant de l'extrémité du ligament; enfin, s'atténuant en une partie rostrale excessivement obtuse-arrondie, un tout soit peu inférieure.

Sommets convexes, non proéminents (toujours excoriés). Arête dorsale confondue dans la convexité, pourvue d'un semblant de sillon à sa partie supérieure. Crête peu comprimée.

Stries ordinaires, çà et là plus saillantes, très feuilletées vers les contours. Épiderme d'un beau vert d'eau, passant vers les sommets à une teinte marron-brunâtre plus ou moins foncée. Intérieur d'une nacre blanche-bleuacée peu irisée.

Ligaments : antéro-interne très robuste et très antérieur; postérieur plus allongé que celui de la sedentaria, à moitié recouvert et terminé par une lunule nettement triangulaire. Charnière légèrement ondulée.

Cette Anodonte, dédiée au $\mathrm{D}^{\mathrm{r}}$ Ludwig Spengler d'Ems, vit dans la Lahn, entre Ems et Dossenau (ancien grandduché de Nassau), en Allemagne. En France, elle a été trouvée dans la Loire à Villeret (Locard), et, dans la Rance à Dinan près Saint-Malo (Mabille).

Les échantillons de la Rance constituent une variété major (long. 102, haut. max. 58, épaiss. 37 millim.), à valves assez épaisses, d'une nacre intérieure blanche et d'un épiderme d'un brun marron uniforme. 


\section{Anodonta Preimeri.}

Cette espèce, qque j'inscris sous ce nom, en l'honneur du malacologiste C. Pfeifler, est l'Acéphale que cet auteur a fait représenter (nat. Deutsch. Moll. 11, 1825, pl. III, f. 1-3 seulement) comme une ventricosa non adulle. Cette Anoconte (roir ce que j'ai dit ci-dessus, page 120), n'a aucun rapport avec la vraie rentricosa figurée planche III (fig. f). Elle constitue une forme spéciale du groupe des Spengleriana.

La Pfeifferi vit aux environs de Cassel, dans l'ancienne Hesse électorale et, au sud du IIanovre, dans les rivières de la petite principauté de Schaumbourg-Lippe.

\section{Anodonta Servaini.}

Petite Anodonte subobovale, ou plutôt de forme subqua. drangulaire, par suite de la grande dilatation en hauteur de sa région postérieure, et de la réduction de sa région antérieure. Cette espèce ressemble un peu, en écourté, à la Lusitana du Portugal, tout en possédant un certain air de rapprochement avec les $P$ iscinalis. Sa convexité a son maximum presque à égale distance des sommets, de l'angle et de la base de la perpendiculaire. Ses valves d'une couleur sombre, assez minces, sont très bâillantes en arrière, entre l'angle et le rostre, et, dans toute l'étendue de ses contours antérieur et palléal.

Long $\max . \ldots \ldots \ldots \ldots \ldots \ldots \ldots . .6 \%$ mill.

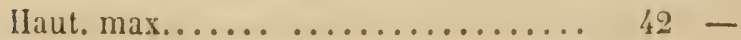

Epaiss, max. (à 20 des sommels, à 37 du 
rostre, à 30 du bord antér., à 21 de l'angle postéro-dorsal, à 22 du la base de la perpend.)................

Long. de la crête ligam.-dors., des sommets à l'angle postéro-dorsal...... 26 -

Dist. de cet angle au rostre......... 30̈ -

Corde apico-rostrale............. 55 -

Haut. de la perpendiculaire......... 37 -

Dist. de cette perpend. au bord antér... 21 -

- du même point de cette perpend. au rostre.....................

- enfin, de la base de la perpend. à l'angle postéro-dorsal.........

24 mill.

$47-$

$42-$

Bord supérieur rectiligne, puis, à partir de l'angle, descendant obliquement en ligne droite sur le rostre. Région antérieure fort peu dilatée en hauteur et fortement décurrente à la base. Bord inférieur descendant d'une façon presque rectiligne jusqu'à 10 mill. en arrière de la perpendiculaire, puis formant ventre, et remontant ensuite vers le rostre. Région postérieure, plus de deux fois plus longue que l'antérieure, allant en se dilatant en hauteur jusqu'au niveau de l'extrémité du ligament, enfin s'atténuant, ensuite, pour se terminer par une partie rostrale très obtuse.

Sommets convexes, non proéminents (toujours excoriés). Arête dorsale accusée seulement par une large radiation plus verte. Crête peu développée, assez comprimée vers l'angle.

Stries régulières, assez fines, très feuilletées vers les contours. Épiderme d'une teinte sombre verdâtre, plus foncée sur les régions dorsales de l'arête et de la crête, et vas 1881. 
s'éclaircissant vers les sommets. Intérieur d'une belle nacre blanche bleuacée.

Ligaments: antéro-interne filiforme; postérieur, médiocre, à moitié recouvert, terminé par une longue lunule allongée. Région cardinale de la charnière nulle. Région latérale relativement très épaisse.

Cette Anodonte, que je dédie à notre ami le docteur G. Servain, habite la Lahn, au-dessus d'Ems, dans l'ancien duché de Nassau, en Allemagne.

\section{Anodonta Hazayana.}

Anodonta Hazayana, Servain, Hist. mal. Balaton (s. p.), 1881.

Petite espèce (long. 69, haut. 41, ép. 26) d'une forme oblongue peu allongée, remarquable par la grande convexité de son arête dorsale. Chez cette espèce, le maximuin de la convexité, peu éloigné (16 mill.) de l'angle postérodorsal, est à égale distance (3๖ mill.) du rostre et du bord antérieur. Les bords supérieur et inférieur, recto-descendants, sont parallèles sur une longueur de près de 30 millimètres.

Bords du lac Balaton, en Hongrie (Servain).

\section{Anodonta minima.}

Anodonta minima, Millet, desc. deux nouv. esp. Anod. in : Mém. soc. agric. sc. d'Angers. 1 (3 livr. 1833), p. 241, pl. XII, f. 2.

Jolie petite Anodonte, bien caractérisée, des ruisseaux 
qui se jettent dans l'Oudon, arrondissement de Segré, dans le Maine-et-Loire (Millet).

Je la connais du Maine à Angers (Servain), du canal d'Ille-et-Vilaine, près de Rennes (Nlabille), du canal entre Loir-et-Cher (Rambur), de la Masle à Amboise (Mabille), du Ternin à Autun et de l'Yvette à Orsay, près Paris. (Coutagne.)

La variété B de l'Anodonta intermedia de Lamarck, dont la représentation se trouve dans les Flussconchylien de Schröter (pl. 1, fig. 3), est une forme très voisine de la minima.

\section{Anodonta Iricassina.}

Anodonta tricassina, E. Pillot, in litt.

Petite Anodonte voisine de la minima, mais en différant par ses valves plus minces, non bâillantes (celles de la minima sont plus fortement entrebâillées en avant et en arrière au-dessus du rostre); par ses striations plus délicates, moins feuilletées vers les contours; par son épiderme clair d'un cendré-carnéolé, passant à une nuance verdâtre radiée entre l'angle postéro-dorsal et le rostre; par sa convexité beaucoup moins forte (la minima a ordinairement 19 à 20 d'épaisseur ; la tricassina n'a que 14 à 15 mill., ce qui fait paraitre ses valves presque aplaties), par le maximt'in de la convexité plus rapproché des som. mets (celui de la minima, à peu près central, est plus distant des sommets); par sa crête dorsale plus comprimée, pourvue d'un angle postéro-dorsal taillé carrément et non émoussé comme celui de la minima; par son bord infé- 


\section{$-324-$}

rieur plus convexe; par son rostre plus obtus, plus amplement arrondi; par sa région postérieure plus dilatée en hauteur et moins développée en longueur; enfin, par sa forme non oblongue, mais ovalaire, etc.

Voici la mensuration de la tricassina :

Long. $\max . \ldots \ldots \ldots \ldots \ldots \ldots \ldots$. 2 2 mill.

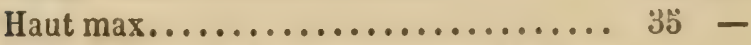

Épaiss. max. (à 11 des sommets, à 32 du rostre, à 20 du bord ant., à 18 de l'angle postéro-dorsal, à 20 de la base de la perpendiculaire).............. 15 -

Long. de la crête ligam.-dors., des sommets à l'angle postéro-dorsal........ 19 -

Dist. de cet angle au rostre........... 28 -

Corde apico-rostrale............. 41 -

Haut. de la perpendiculaire.......... 31 -

Dist. de cette perpend. au bord antér.... 16 -

- du même point de cette perp. au rostre. 35 -

- enfin, de la base de la perpend. à

l'angle postéro dorsal......... 35 -

La tricassina a été recueillie aux alentours de Troyes (Aube), dans divers bras de la Seine, notamment dans celui de Groncels, vers le déversoir. (Pillot.)

\section{PICARDIANA}

Les Picardiana sont des Anodontes intermédiaires entre les Spengleriana et les Piscinaliana, de forme ovoide, à convexilé bien régulière normalement répartie, à arête dor- 
sale peu prononcée, ou nulle, recto-descendante sur une partie rostrale inférieure, jamais ascendante. Ces Anodontes vivent dans les mêmes conditions que les Spengleriana.

\section{Anodonta Plerraí.}

\begin{tabular}{|c|c|c|}
\hline & & \\
\hline Haut. $\max . . .$. & 48 & - \\
\hline $\begin{array}{l}\text { pais. max. (à } 24 \text { des sommets, à } 40 \text { du } \\
\text { rostre, à } 36 \text { du bord antér., à } 24 \text { de } \\
\text { l'angle póstéro-dorsal, à } 26 \text { de la base } \\
\text { de la perpend.).................. }\end{array}$ & 28 & - \\
\hline $\begin{array}{l}\text { Long. de la crête ligamento-dorsale des } \\
\text { sommets à l'angle postéro-dorsal... }\end{array}$ & 31 & - \\
\hline rostre.......... & 38 & - \\
\hline Cord & 3 & - \\
\hline ...... & 45 & - \\
\hline $\begin{array}{l}\text { antér...... } \\
\text { perpend. au }\end{array}$ & 24 & - \\
\hline$\cdots$ & & \\
\hline
\end{tabular}

Jolie Anodonte, à convexité bien régulière, presque cen. trale; à valves relativement épaisses, brillantes, pourvues d'un seul iâillement entre le rostre et l'angle postérodorsal.

Bord supérieur rectiligne jusqu'à l'extrémité du ligament, puis subissant une légère inflexion au niveau de la lunule, enfin, descendant obliquement sur le rostre à partir de l'angle postéro.rostral, qui est fort obtus. Région antérieure assez ample, bien arrondie, néanmoins un tant 
soit peu décurrente à la base. Bord inférieur régulièrement convexe jusqu'au rostre. Région postérieure un peu plus de deux fois plus longue que l'antérieure, augmentant fort peu en hauteur jusqu'au niveau de l'extrémité du ligament, puis s'atténuant en une partie rostrale inférieure, obtuse-arrondie.

Sommets non proéminents, bien que convexes-ventrus, sillonnés par de très fines stries tremblotées. Crochets très aigus. Arête dorsale confondue dans la ventrosité des valves, accusée seulement par deux radiations vertes plus larges. Crête dorsale très médiocre, à peine comprimée.

Stries délicates, un peu saillantes et feuilletées seulement vers la circonférence. Epiderme brillant, d'un cendré-verdâtre ou d'un vert-jaunacé s'éclaircissant vers les sommets et se fonçant vers les contours, orné, en outre, sur la région postérieure, de quelques radiations vertes, plus larges sur l'arête dorsale, et ensuite, si pressées les unes contre les autres, entre cette arête et le contour de la crête dorsale, qu'elles ne paraissent ne faire plus qu'une seule bande. Intérieur d'une nacre blanchâtre assez mate.

Ligaments : antéro-interne épais, occupant toute l'épaisseur de la cardinale; postérieur robuste, presque entièrement recouvert. Lunule longue de 10 mill. Impressions antérieure profonde; postérieure superficielle.

Cette espèce, dédiée à M. C. Picard, auteur de l'Histoire des Mollusques du département de la Somme, vit dans l'Escaut, à Valenciennes, d'où elle m'a été adressće sous le nom fautif de Scaldiana. 


\section{Anodonta Journei.}

Anodonta Journei, Ray, in litt.

La Journei, plus allongée que la Picardi, a une convexité relativement moins prononcée; sa partie rostrale, moins obtuse, est plus développée; son bord supérieur est exactement rectiligne; sa région antérieure, sensiblement plus grande, est plus décurrente à la base; ses sommets sont moins ventrus; ses striations, moins délicates, sont çà et là saillantes et surtout plus feuilletées vers la circonférence son épiderme est moins brillante; enfin, ses valves, pourvues d'un bâillement plus considérable entre le rostre et l'angle postéro-dorsal, offrent, en outre, un autre bâillement, réduit à l'état de fente, à la partie inféro-antérieure.

Long. $\max \ldots \ldots \ldots \ldots \ldots \ldots \ldots \ldots, 8$ mill.

Haut. $\max . . . \ldots \ldots \ldots \ldots \ldots \ldots . . . .68$.

Épaiss. max. (à 22 des sommets, à 47 du rostre, à 38 du bord antér., à 28 de l'angle postéro-dorsal, à 27 de la base de la perpendiculaire)............. 28 -

Long. de la crête ligam.-dors., des sommets à l'angle postéro-dorsal....... 33 -

Dist. de cet angle au rostre........... 38 -

Corde apico-rostrale.............. 6ə -

Haut. de la perpendieulaire.......... 45 -

Dist. de cette perpend. au bord antér.... 28 - 
Dist. du même point de celte perpend. au rostre.................. 57 mill.

- enfin, de la base de la perpend. à l'angle postéro-dorsal......... 52 -

Bord supérieur commençant brusquement à 14 mill. en avant des sommets, où, il forme un angle bien accentué en haut du contour antérieır, puis se poursuivant d'une façon nettement rectiligne jusqu'à l'angle postéro. dorsal, pour de là descenảre obliquement en ligne droite sur le rostre. Région antérieure grande, bien arrondie, décurrente à la base. Bord inférieur régulièrement convexe dans toute son étendue, seulement à un degré moindre que celui de la $\boldsymbol{P}$ icardi. Région postérieure deux fois plus longue (à 1 mill. près) que l'antérieure, augmentant fort peu en hauteur, jusqu'à l'extrémité du ligament, puis s'atténuant en un rostre inférieur formant un peu bec, subarrondi ou bien un tant soit peu subtroncatulé.

Sommets peu convexes, non proéminents, sillonnés de très fines striations tremblotées. Grochets aigus, saillants. Arête dorsale confondue dans la convexité, accusée seulement par une large radiation noir-olivâtre ou vertes'étendant jusqqu'au ligament. Grête médiocre, peu comprimée, néanmoins un peu plus que celle de la Picardi.

Stries irrégulières, çà et là délicates, puis saillantes. quelquefois grossières, devenant plus fortes et feuilletées vers la circonférence. Epiderme ì peu près du même coloris que celui de la Picardi, seulement moins brillant. Intérieur d'une nacre blanchâtre, àssez irisée.

Ligaments: antéro-interne filiforme; postérieur robuste, presque entièrement symphynoté. Lunule très développée. 
Cette Anodonte, que le savant zoologiste J. Ray, de Troyes, a dédiée à H. Camille Journé, entomologiste troyen, a été recueillie dans l'Aube, à Bar-sur-Aube.

Notre ami J. Mabille a rencontré cette même espèce dans la Nonette à Chaalis près d'Ermenonville(0ise). Les échan. tillons de cette localité, bien semblables à ceux de l'Aube, peuvent constituer une variété major (long. 108, haut. max. 62, haut perpend. 59, épaisseur max. 39 mill.).

\section{Anodonta Dubreili.}

Anodonta Dubreili, Servain, Hist. malac. Balaton (s. pr.), 1881.

Cette belle espèce (long. 92, haut. max. 5\$, haut. perpend. 56, ćpaiss. max, 36 mill.), d'une teinte uniforme couleur noisette, est relativement très haute pour sa taille. Sa région antérieure, exactement sphérique, est largement développée; sa région postérieure pas tout à fait deux fois plus longue que l'antérieure, se termine par une partie rostrale inférieure non obtuse, mais d'un contour arrondi assez aigu; la convexité bien régulière, sauf entre l'arête et la crête dorsale où elle descend assez brusquement, est presque centrale, à quelques millimètres près; l'arête dorsale est limitée supérieurement par un sillon accentué, d'une nuance plus foncée; l'angle postéro-dorsal est très en contre-bas du ligament par suite de la direction obliquedescendante de la lunule; les valves, bien brillantes, déli. catement striolées, sauf vers la circonférerce, sont pourvues de deux bâillements, dont l'un très peu ouvert s'étend sur toute l'étendue des contours antérieur et inférieur, et, 
l'autre, plus ouvert, se montre entre le rostre et l'angle postéro-dorsal.

Lac Balaton, en Hongrie. (Servain.)

\section{Anodonta Monterosari.}

La Monterosati est une forme oblongue ovoïde, à convexité d'une grande régularité. Ses bords supérieur et inférieur sont à peu près aussi bien arqués l'un que l'autre, et ses valves, assez épaisses, offrent un énorme bâillement qui s'étend dans toute l'étendue du contour depuis le commencement antéro-supérieur jusqu'au rostre.

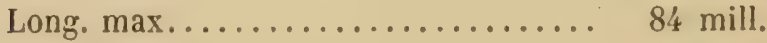

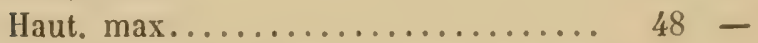

Épaiss. max. (à 27 des sommets, à 40 du rostre, à 44 du bord antér., à 23 de l'angle postéro-dorsal, à 28 de la base de la perpendiculaire.)............

Long. de la crête ligamento-dorsale, des sommets à l'angle postéı o-dorsal.....

Dist. de cet angle au rostre......... 40 -.

Corde apico-rostrale............ 65 -

Haut. de la perpendiculaire......... 47 -

Dist. de la perpend. au bord antér... 29 -

- du même point de cette perpend. au rostre............... $56 \quad-$

- enfin, de la base de la perpend. à l'angle postéro-dorsal....... 50 -

Bord supérieur offrant une convexité presque parfaite dans toute son étendue, depuis son extrémité antérieure 
jusqu'au rostre, sauf à l'endroit de l'angle postéro-dorsal, où, il montre une légère partie anguleuse. Région antérieure de forme ovoïde: Bord inférieur régulièrement convexe dan; tout son parcours. Région postérieure pas tout à fait (moins 2 millimètres) deux fois plus longue que l'antérieure, augmentant à peine en hauteur, s'alté. nuant à partir du niveau de l'angle postéro-dorsal en un rostre très obtus-arrondi, un peu moins inférieur que celui des autres espèces de ce groupe.

Sommets convexes, peu proéminents, toujours excoriés, malgré tout, laissant voir sur la nacre sous-épidermique de furts sillons concentriques bien espacés. Crochets aigus. Arête nulle, confondue dans la convexité. Crête très réduite, peu comprimée.

Stries tantôt délicates, tantôt saillantes, devenant plus grossières vers la circonférence. Épiderme recouvert d'un enduit noirâtre très tenace, et, lorsqu'il est enlevé, montrant une sulface brillante d'un ton jaunacé-oliveâtre s'éclaircissant vers les sommets. Intérieur d'une nacre blanche assez bien irisée.

Ligaments : antéro-interne très épais au niveau des crochets; postérieur robuste, saillant et presque entièrement symphynoté. Lunule étroite, allongée. Impressions profondes : l'antérieure, oblongue; la postérieure, en forme de palette.

Ciette espèco, que je dédie à notre ami le marquis de Monterosato de Palerme, habite le lac de Pérouse, où elle a été recueillie, à une heure de distance environ, au no: $d$ de Passignano. 


\section{Anodonta Maritzana.}

Jolie petite Anodonte, d'une forme ovoille plus allongée que toutes celles de ce groupe, d'une convexité également moins forte, et, caractérisée, sans compter un très faible entrebâillement entre le rostre et l'angle postéro-dorsal, par un autre considérable, s'étendant sur tout le parcours antérieur et palléal du sommet au rostre.

\begin{tabular}{|c|c|c|}
\hline & & \\
\hline Iaut. $\max . . . .$. & & \\
\hline 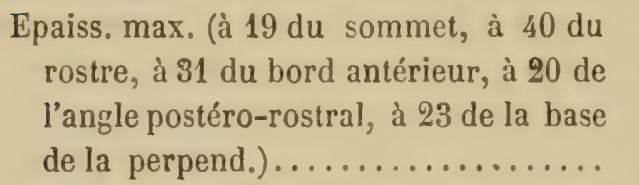 & & \\
\hline $\begin{array}{l}\text { Lung. de la crête ligam.-dors., des sommets } \\
\text { à l'angle postéro-dorsal............ }\end{array}$ & & \\
\hline 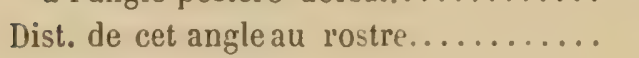 & & \\
\hline$\ldots . . . \ldots \ldots \ldots$ & & \\
\hline$\ldots \ldots \ldots$ & 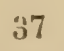 & \\
\hline $\begin{array}{l}\text { Dist. de la perpend. au bord antér..... } \\
\text { - du même point de cette perpend.au }\end{array}$ & 20 & \\
\hline $\begin{array}{r}\text { rostre.. } \\
\text { - enfin, de }\end{array}$ & & \\
\hline
\end{tabular}

Bord supérieur faiblement arqué jusqu'à l'angle, puis recto-descendant sur le rostre. Région antérieure bien ronde. Bord inférieur régulièrement convexe dans toute son étendue. Région postérieure deux fois et demie plus 
longue que l'antérieure, augmentant fort peu en hauteur jusqu'd 22 millimètres en arrière de la perpendiculaire, puis allant en s'atténuant en un rostre obtus-arrondi, un peu inférieur.

Sommets convexes, à peine proéminents, sillonnés par des rides irrégulières, très saillantes, ressemblant à des rugosités. Grochets aigus. Arète presque nulle, seulement indiquée par une coloration plus foncée. Crête fort médiocre, peu comprimée.

Stries très délicates, saillantes et feuilletées seulement vers la circonférence. Épiderme brillant, d'une belle teinte jaune devenant cendrée sur la région ombonale et noir-verdâtre entre l'arête et la crête. Intérieur d'une belle nacre blanchâtre irisée de toutes les couleurs de l'arc-en-ciel.

Ligaments : antéro-interne, filiforme; postérieur, allongé, médiocre. Lunule triangulaire. Région latérale de la charnière très épaisse. Région cardinale nulle.

Cette anodonte vit dans les eaux de la Maritzi, près d'Andrinople, en Rumélie.

\section{จ}

Les Acéphales que je réunis dans ce groupe, sont tous caractérisés à un degré plus ou moins prononcé, suivant les espèces, par un mode de convexité se développant sur la région postérieure, sous une apparence cintréeconcave, et, par une partie rostrale plus ou moins allongée sous une tendance remontante.

Ghez les Piscinaliana, le maximum de convexité se trouve ordinairement porté sur la région ombonale. 
Les :spèces de ce groupe vivent dans les lacs, les fleuves ou les rivières à fond vaseux.

\section{Anodonta piscelnalis.}

Anodonta piscinalis (pars), Nilsson, Moll. Sueciœ, p. 116, 1822, et, Rossmässler, Iconogr. IV, p. 25, fig. 281, 1836 (non, piscinalis, fig. 416, qui doit être rapportée à la Dantessantyi de Ray).

Cette piscinalis est encore une de ces espèces inconnues, à laquelle on a rapporté des quantités de formes très dissemblables.

Nilsson, l'auteur de l'espèce, a donné à sa piscinalis les caractères suivants :

\& Testa elliptico-ovata, ventricosa, subradiata; anterius rotundata, posterius producta, angulata ; pube compressa, aurita; natibus prominulis, coindutis, ab extremitate remotis; umbonibus tumidis; Diam. transv. 105, long. 60 mill, $\triangleright$ et, cet auteur cite comme synonyme de son Anodonte :

$1^{\circ}$ Le Mytilus cygneus de Schröter (flussconch., p. 150, pl. 3, f. 1). "Quin, dit Nilsson (p. 117), Rev. Schröter, nostram speciem descripserit et delineaverit nulli omnino dubitamus ";

$2^{\circ}$ L'Anodonta anatina de Draparnaud (IIoll. France, pl. XII, f. 2), et il ajoute : "figura Draparnaldi citata, sub nomine Anodonlce anatine, formam nostrœ speciei egregie exprimit, sed minor est nostris speciminibus v;

$3^{\circ}$ L'Anodonta trapesialis de Lamarck (An. s. vert. VI, première partie, p. 87? Chemnitz, Conch., t. 86, f. 762 ? 
- Cette dernière synonymie est indiquée avec doute. Nilsson avoue, du reste (p. 117), an vero Anod trapesialis ad quam illustrandam Schroteri adfertur, cum figura Chemnitzii ibidem allata dubia nobis videtur, et ex encycloped. citatas icones consulere non possumus, cum liber in nulla bibliotheca, nec publica nec privata, apud nos exstat $).$

Nilsson a eu raison de considérer avec doute la trapesialis comme semblable à son espèce, attendu que cette Anodonte du Brésil ra pas le moindre rapport avec la piscinalis d'Europe, comme on peut s'en convaincre en étudiant la figure $1 A$ et $B$ de la planche 205 de l'Encyclopédie, ou celle que Kuster (Anod., p. 31, pl. VIII, f. 4, 1852) a donné de cet Acéphale.

En éliminant cette synonymie, qui n'a pas sa raison d'être, il en reste deux autres : celle de-Schröter et celle de Draparnaud.

Or, lorsqu'on examine la figure 1 de la planche 3 de Schröter, on reconnaît une forme ovalaire presque subquadrangulaire, très haute de taille en avant et en arrière, à région postérieure plus ample, plus dilatée que l'antérieure, se terminant par une partie rostrale excessivement écourtée, et n'ayant aucun rapport de contour et d'aspect avec la piscinalis représentée (fig. 281) par Rossmässler, d'après des échantillons de Nilsson, échantillons bien rostrés et à région postérieure bien allongée.

Je ferai, en outre, remarquer qu'un des caractères de la piscinalis est d'av ir les sommets antérieurs, tandis que ceux de l'espèce figurée par Schröler, espèce qu'il convient de rapporter à mon exocha du Rhône, sont assez médians.

Quani à cetle autre Anodonte, représentée (pl. XII, f. 2) 
dans l'ouvrage de Draparnaud, sous le i:on d'anatina, Anodonte qque Nilsson considère comme le type, en plus petit, de sa piscinalis; j'avoue n'y plus rien comprendre : $1^{\circ}$ parce que cetle forme, qui ne me paraît pas adulte, ne possède pas les caractères des piscinales; $2^{\circ}$ parce qu'elle ne ressemble aucunement à cet échantillon représenté (pl. 3, fig. 1) par Schroter, échantillon que Nilsson regarde également comme identique à sa piscinalis. Je prie instamment les malacologisies de faire la comparaison.

En somme, sous le nom de piscinalis, il y a au moins trois formes distinctes :

$1^{\circ}$ La piscinalis de la description qui possède une région postérieure allongée (producta) et rostrée;

$2 \cdot$ La soi-disant piscinalis des flussconchyliens de Schröter, à région postérieure plus dilatée en hauteur que la région antérieure, et à rostre très écourté;

$3^{\circ}$ Cette autre soi-disant anatina de l'ouvrage de Draparnaud, qui n'appartient point au groupe des Piscinaliana.

Pour la trapesialis de Lamarck, rapprochée aussi de l'espèce suédoise, il n'en faut pas parler. L'erreur est trop évidente.

En résumé, si l'on avait que le travail de Nilsson, on serait bien embarrassé de savoir ce que peut être la piscinalis. Heureusement qu'on a l'inconographie de Rossmässler.

Je considère Rossmässler comme le véritable créateur de cette espèce, parce que la figure qu'il a donnée de cette espèce (fig. 281), d'après des échantillons envoyés par Nilsson, cadre bien, pour les caractères, avec ceux que l'auteur suédois a attribué à son Acéphale dans la description que j'ai citée. 
La piscinalis de la figure 281 est une forme à région postérieure allongée et nettement rostrée, à rostre un tant soit peu ascendant. Chez cette espèce, le bord supérieur, presque rectiligne jusqu'à l'angle postéro-dorsal, descend ensuite sur le rostre par un contour concave; le bord inférieur est régulièrement très convexe; l'arête dorsale se développe sous une direction un tant soit peu cintrée-concave; la crête est ample, assez comprimée, avec un angle carrément coupé. Le seul caractìre, qui laisse à désirer dans cette figure 281, est celui de la ventrosité de la région ombonale, ventrosité qui n'est pas suflisamment accusée. Chez la vraie piscinalis, les umbones sont toujours très renflés et la convexité des valves est reportée vers eux. C'est le défaut de cette figure qui, par le manque d'ombre, paraît représenter une espèce presque aplatie.

Voici la mensuration de la piscinalis, d'après un échantillon du Danube, échantillon tout à fait semblable à la figure 281 de Rossmässler, sauf une taille un tant soit peu plus forte.

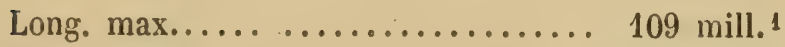

Haut. $\max . . . \ldots \ldots \ldots . . . . . .6 .65-$

Épaiss. max (à 32 des sommets, à š9 du rostre, à 50 du bord antér., à 32 de l'angle postéro-dorsal, à 36 de la base de la perpend.)................

Long. de la crête ligam.-dors., des sommets à l'angle postéro-dorsal.......

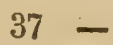

$38-$

1. 105 mill, d'après Nilsson; la figure 281, de Rossmässler, a 103 mill.

MAI. 1881. 
Distance de cet angle au rostre...... 5 51 mill.

Corde apico-rostrale............ 80 -

Haut. de la perpendiculaire......... 64 -

Dist. de cette perpend, au bord antér... 30 1/2

- du même point de cette perpend. au rostre................. 75 -

- enfin, de la base de la perpend. à l'angle postéro-dorsal........... 67 -

D'après ces mensurations, on remarque : $1^{\circ}$ que la convexité est moins distante des sommets et de l'angle postéro-dorsal que de la base de la perpendiculaire; $2^{\circ}$ que la région antérieure est plus de deux fois plus développée que l'antérieure; $3^{\circ}$ que la longueur de la crête est courte en comparaison de celle de l'angle au rostre (38 contre 51 mill.), ce qui dénote une région postérieure allongée; $4^{\circ}$ que la convexité ( 37 mill.) est relativement très forte, puisqu'elle atteint presque le tiers de la longueur et plus de moitié de la hauteur; $5^{\circ}$ enfin, que la région postérieure augmente seulement de un millimètre en hauteur.

Celte Anodonte vit en Suède et en Allemagne. Je la possède de différentes localités, notamment du Danube. Je ne la connais pas dans notre pays, bien qu'elle y ait été indi. quée nombre de fois.

Les auteurs, du reste, ont eu, chacun, en vue une forme dilférente, comme l'on peut s'en convaincre en comparant soit leurs descriptions, soit les figures qu'ils ont donné de cette espèce, avec celle (281) de l'Iconographie.

Ainsi : la piscinalis (fiğ. 416) du même ouvrage de Rossmäisler, est une forme très distincte que je rapporte à la Dantessantyi de Jules Ray; 
Celle de 11. Drouët (Naïad. France, pl. V, f. 1) est la subrhombea, de Brown;

Celle de Brot (Naïad. Léman, pl. V, f. 1) est l'opalina de Kuster;

Celle de Gassies (Moll. agenais, pl. IV, f. 1, 1849) rappelle la Dupuyi de Ray;

Etc., etc.

Enfin, celle des Suites à Rossmässler (fig. 19:09) est une forme qui n'appartient même pas au groupe des Piscinaliana, mais à celui des Arealiana, et qui me parait voisine de la Kusteri (voir page 284).

\section{Anodonta opalina.}

Anodonta opalina Küster, Anod. (2édit., Chemnitz), p. 60, pl. XVI, fol. 1.2, 1852).

Anodonta radiata, var. A. A. Mörch, Syn. Moll. Danice, p. $86,1864$.

Anodonta piscinalis, Brot, Nayacies Léman, p. 47, pl. V, f. 1, 1867.

Küster signale le type de l'opalina dans un étang, près d'Erlangen, en Bavière.

Brot indique, en Suisse, cette Anodonte sous le nom de Piscinalis, à Fernex, près de la tuilerie Grobet, dans des mares formées par d'anciennes excavations faites pour l'exploitation de la terre à pot.

Mörch, sous l'appellation de radiala, me l'a envoyée du Danemark, sans indication de localité.

Je l'ai reçue de Pologne, sous le nom d'anatina, comme provenant de la Vistule; enfin, notre ami le conseiller 
Letourneux a recueilli de fort beaux exemplaires de l'opalina, dans le Danube, à Belgrade (Serbie) et à Giurgewo (Valachie).

Je renvoie pour la connaissance de cette espèce aux excellentes et très exactes figures données par Brot et par Küster.

\section{Anodonta Savica.}

Anodonta Savensis, Drouël, in : Journ. Conch., p. 28, 1881.

Cet Acéphale est, à ce qu'il paraît, abondant dans la Save et ses affluents, notamment à Usce, dans le marais de Grabovac et dans le Kamicak, près de Schabatz, en Serbie.

J'ai modifié l'appellation imposée à cette espèce, attendu que la désinence ensis ne convient pas à un nom de fleuve ou de rivière.

\section{Anodonta Kickxi.}

Anodonta Kickxi (excl, var.) Colbeau, exc. mal. Belg. in : ann. soc. malac. Belg. I, p. 60, 1865, et Moll. Belg. in : ann. soc. malac. Belg. III, 1868, p. 107, pl. III, f.1, et Clessin, Anod. (2०édit. Chemnilz), p. 228. pl. LXX, f. 4, 1876.

Colbeau rapporte à cette espèce, qui est fort remarquable : $1^{\circ}$ l'Anod. ponderosa de Kickx (syn. moll. Brab. 1830 , p. 81) ; $2^{\circ}$ l'Anod. subponderosa de Colbeau (matér. 
faune malac. Belg, 1859, p. 12), et de Malzine (essai faune malac. Belg. 1867, p. 35); $3^{\circ}$ l'Anod. cygnea, var. de Lecomte, in: ann. soc. malac. de Belgique.

La Kickxi paraît répandue dans un assez grand nombre de localités belges, notamment dans les étangs de Perck, près de Louvain.

\section{Anodonta ilysoeca.}

Espèce ovalaire dans une direction descendante, caractérisée par une convexité postérieure légèrement cintréeconcave, à valves lisses, bien brillantes seulement sur la région ventrale, et à stries très feuilletées sur tout le reste de leur étendue, n'offrant, en outre, qu'un seul entre-bâlllement assez ouvert entre le rostre et l'angle postérodorsal.

Long. $\max . \ldots \ldots \ldots \ldots \ldots \ldots \ldots . \ldots . \ldots . \ldots$ mill.

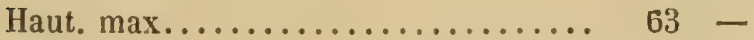

Épaiss. max. (à 28 des sommers, à 60 du rostre, à $38 \mathrm{du}$ bord antér., à 34 de l'angle postéro-dorsal, à 31 de la base de la perpend.).................

Long. de la crête ligam.-dors., des sommets à l'angle postéro-dorsal...... 38 -

Dist. de cet angle au rostre......... 46 -

Corde apico-rostrale............. 79 -

Haut. de la perpendiculaire......... 58 -

Dist. de la perpend. au bord antér..... 31 -

- du même point de cette perpend. au rostre..................

- enfin, de la base de la perpend. à l'angle postéro-dorsal...... 60 - 
Bord supérieur convexe jusqu’à l'extrémité du ligament, puis subiscant une inflexion à l'endroit de la lunule, enfin descendant sur le rostre dans une direction oblique, presque rectiligne, malgré tout un tant soit peu concave. Région antérieure arrondie, décurrente à la base. Bord inférieur convexe-descendant jusqu'd 294 mill. en arrière de la perpendiculaire (point où la convexité est la plus accentuée), puis, remontant par une courbe régulière vers le rostre. Région postérieure plus de deux fois plus longue que l'antérieure, augmentant en hauteur (par suite de la grande convexité du bord palléal) jusqu'au niveau de l'extrémité du ligament, enfin, allant en s'atténuant en un rostre obtus, arrondi, ayant une tendance ascendante.

Sommets ventrus, gonflés, bien que non proéminents, sillonnés seulement par quelques striations excessirement fines et serrées. Crochets très aigus, saillants. Arête dorsale faiblement concave, confondue dans la convexité qui, en cet endroit, s'abaisse en pente régulière sur la crête, enfin, limitée supérieurement par un léger sillon venant aboutir à la partie supérieure du rostre. Grête très courte, peu développée, assez comprimée.

Stries trè̀ fines sur les régions ventrale et ombonale qui paraissent presque lisses, devenant ensuite, sur une large étendue de la circonférence, saillantes et très feuilletées. Epiderme brillant, d'une belle teinte jaune-verdâtre faiblement radiée, passant au cendré violacé vers les sommets. Intérieur d'une belle n:.cre blanche bleuacée très irriséte.

Ligaments : antéro-interne médiocre, très allongé ; postérieur court, très saillant et tout à fait symphynoté. Lunule fort longue (12 mill.), subtriangulaire. Région latérale de la charnière très épaisse. Région cardinale nulle. 
Cette Anodonte a été recueillie dans le Danube, à Giurgewo Valachie) par le conseiller Istourneux.

\section{Anodonta scaphidella.}

Anodonta scaphidella, Letourneux, in litt.

Acéphale de forme plus oblongue dans une direction faiblement descendante, remarquable par sa partie rostrale, inférieure et en forme de bec. Chez, cette espèce, les valves offrent deux entre-bâillements considérables, l'un sur toute l'étendue des contours antérieur et inférieur; l'autre entre le rostre et l'angle postíro-dorsal.

Long. $\max . \ldots \ldots \ldots \ldots \ldots \ldots \ldots . . .62$ mill.

Haut. $\max . . . \ldots \ldots \ldots \ldots \ldots \ldots$. 55

Épaiss. max. (à 22 des somıets, à 57 du rostre, à $36 \mathrm{du}$ bord antér., à 28 de l'angle postéro-dorsal, à 3.1 de la base de la perpendiculaire).......... 33 -

Long. de la crête ligam.-dors., des summ.

à l'angle postéro-dorsal.......... 32 -

Dist. de cet angle au rostre......... 47 -

Corde apico-rostrale............. 74 -

Haut. de la perpendiculaire......... 51 -

Dist. de la perpend. au bord antér..... 28 -

- du même point de cette perpend. au rostre .............. 66 -

- enfin, de la base de la perpenıl. à l’angle postéro-dorsal....... วั4 - 
Je ferai remarquer la grande différence (15 mill.) qui existe entre la longueur de la crête (32 mill.) et celle de l'angle au rostre (47 mill.), lorsque, chez l'espèce précédente, qui est pourtant d'une taille plus forte, la différence n'atteint que 8 mill.. Ce grand écart, entre les deux longueurs de la scaphidella, provient du fort allongement de la partie rostrale.

Bord supérieur très faiblement arqué jusqu'à l'angle, puis descendant sur le rostre par un contour nettement concave. Région antérieure arrondie, moins décurrente à la base que celle de l'espèce précédente. Bord inférieur exactement convexe dans toute son étendue. Région postérieure plus de deux fois plus longue que l'antérieure, augmentant jusqu'à 23 mill. en arrière de la perpendiculaire, puis s'atténuant, surtout supérieurement, pour se terminer par un rostre allungé, inférieur, en forme de bec arrondi, et bien moins obtus que celui de l'ilysœca.

Sommets ventrus, peu gonflés, non proéminents, sillonnés par des rides concentriques régulières, fortes et bien espacées. Crochets aigus, ordinairement émoussés. Arête confondue dans la convexilé, qui, en cet endroit, comme chez l'ilysøeca, descend en pente régulière sur la crête, qui est peu développée et à peine comprimée.

Stries assez irrégulières, çà et là saillantes, légèrement fuluilletées vers la circonférence. Epiderme toujours sali d'un enduit terne et verdâtre très tenace, et lorsqu'ıl est enlevé, laissant voir un coloris d'une teinte marron, uniforme avec quelques nuances jaunacées ou olivâtres, enfin, s'éclaircissant'sur les sommets en un ton marron-rougeâtre. Intérieur d'une belle nacre bleuâtre parfaitement irisée.

Ligaments : antéro-interne très antérieur, assez fort 
postérieur volumineux, saillant et symphynoté. Lunule allongée. Charnière latérale épaisse.

Cette Anodonte a été recueillie par le conseiller Letourneux dan la rivière de Krapina-Tœplitz, en Croatie.

\section{Anodonta resima.}

La convexité, chez cette espèce, très portée sur la région ombonale, s'atténue sur la région postérieure sous une tendance cintrée-concave, plus accentuée que chez les Anodontes précédentes. Les valves, assez minces, outre le bâillement postérieur entre l'angle et le rostre, sont fortement entre-bâillées dans toute l'étendue des contours antérieur et palléal 1 .

Long. $\operatorname{mar} . \ldots \ldots \ldots \ldots \ldots \ldots . . . \ldots 4$ mill.

Haut. max.................. 64 -

Épaiss. max. (à 27 des sommets, à $64 \mathrm{du}$ rostre, à 38 du bord antér. et de l'angle post.-fors., à 39 de la base de la perpendiculaire)............... 39 -

Long. de la crête ligam.-dors., des sommets

à l'angle postéro-dorsal.......... 37 -

Di it. de cet angle au rostre.......... 48 1/2

Corde apico-rostrale.............. 81 -

Haut. de la perpendiculaire.......... 60 1/2

Dist. de la perpendicul. au bord antér... 32 1/2

1. Voir pour les contours de la resima, la planche (à l'introduction) de la mensuration des Acéphales. 
Dist. du mème point de la perpend. au rostre................. 71 mill.

- enfin, de la base de la perpend. à

l'angle postéro-dorsal........ 67 1/2

Bord supérieur rectiligne, puis recto-infléchi à l'endroit de la lunule, enfin, descendant sur le rostre par un contour, d'abord concave, ensuite convexe. Région antérieure arrondie, décurrente à la base. Bord inférieur très arqué, présentant sa plus grande convexité à 27 mill. en arrière de la perpendiculaire, puis remontant par une courbe régulière vers le rostre. Région postérieure plus de deux fois plus longue que l'antérieure, angmentant, par suite de la grande convexité du bord inférieur, de 4 mill. en hauteur jusqu'au niveau de l'extrémité du ligament, pour s'atténuer ensuite en une partie rostrale subarrondie, légèrement ascendante.

Sommets ventrus-gonflés, un peu proéminents, sillonnés par de très fines petites rides tremblotées concentriques. Crochets émoussés. Arête dorsale cintrée-concave, non saillante, confondue dans la convexité, qui, de la région ventrale descend en pente régulière sur la crète. Cette crête, assez développée, est comprimée vers l’angle.

Stries excessivement fines et délicates (si délicates mème que la surface parait liss'), devenant saillantes et feuilletées que vers la circonférence. Epiderme presque lisse, très brillant, d'use belle teinte jaune-verdâtre (avec quelques radiations étroites), s'éclaircissant vers les sommets, et se fonçant vers les contours. Intérieur d'une belle nacre irisée bleuâtre.

Ligaments : antéro-interne tout à fait antérieur, ne se prolongeant que de ă mill. en arrière des crochets; posté- 
rieur allongé, entièrement symphynoté. Charnière latérale puissante; charnière cardinale nulle.

Celte belle Anodonte vit dans le Danube aux environs de Giurgewo, en Valachie (Letourneux).

\section{Anodonta falcata.}

Anodonta falcata, Drouët, in : Journ. Conch. 1879, p. 332.

Cet Acéphale habite le Dniéper, à Word-Kodnieprowsk. Il est caractérisé par une région postérieure tellement cintrée-ascendante que l'angle supérieur du rostre atteint le niveau de l'horizon des sommets.

Middendorff (Moll. Sibir, p. 396, 1851), sous l'appellation d'Anod. ventricosa, Var. rostrata, mentionne de la Bessarabie une forme « margine basali jam a medio latitudinis in rustrum surgente » qui pourrait bien n'être autre chose que la falcata.

\section{Anodonta Aristidis .}

Cette Anodonte, que je me fais un plaisir de dédier à notre ami le conseiller Aristide Letourneux, est une forme ovalaire dans une direction légèrement descendante, à convexité, moins gonflée sur la région ombonale, parais. sant sur la région postérieure fort peu cintrée-concave.

Les valves sont fortement entre-bâillées en arrière entre l'angle et le rostre, et en avant, sur toute l'étendue du contour des sommets au ros're. 
Long. $\max . . . \ldots \ldots \ldots \ldots \ldots . . .68$ mill.

Haut. $\max . . . \ldots \ldots \ldots \ldots \ldots .61$. 61 .

Épaiss. max. (à 30 des sommets, à 55 du rostre, à 41 du bord antírieur, à 35 de l'angle postéro-dorsal, à 31 de la base de la perpend.)..............................

Long. de la crête ligamento-dorsale, des sommets à l'angle postéro-dorsal.... 38 -

Dist. de cet angle au rostre.......... 47 -

Corde apico-rostrale.............. 78 -

Haut. de la perpendiculaire......... 60 -

Dist. de cette perpend. au bord antér... 33 -

- du même point de cette perpend. au rostre................... 65 -

- enfin, de la base de la perpend. à l’angle postéro-dorsal......... 61 -

Bord supérieur légèrement arqué, puis subrectiligneincliné des sommets à l'angle, enfin, descendant sur le rostre, à partir de l'angle, dans une direction subrectoconcave. Région antérieure largement arrondie, à peine décurrente à la base. Bord inférieur bien arqué-convexe dans toute son étendue. Région postérieure à peine deux fois plus longue (moins un mill.) que l'antérieure, n'augmentant presque pas en hauteur et s'atténuant, surtout supérieurement, en une partie rostrale inférieure insensiblement ascendante.

Sommets peu ventrus, non proéminents, fort peu silonnés par de petites rides irrégulières. Crochets très aigus. Arête dorsale très faiblement cintrée-concave, confoudue dans la convexité, accusée seulement par une coloration d'un noir-olivâtre, et, sur son extrémité, par un 
sillon assez prononcé. Grête bien développée, subcomprimée.

Stries délicates, très régulières sur toute la région ventrale, devenant plus saillantes vers la circonférence, et feuilletées notamment sur toute la région supéro-postérieure. Epiderme brillant, d'un jaune-paille avec des tons cendrés et rougeâtres vers les sommets, présentant ensuite sur toute la partie supéro-postérieure, de l'arête à la crête, une teinte noir-olivâtre terne, par suite du grand feuilletage des striations. Intérieur d'une nacre blanchebleuacée.

Ligaments : antéro-interne très antérieur, absorbant toute la cardinale qui devient nulle; postérieur médiocre, entièrement symphynoté. Lunule grande, subtriangulaire.

L'A ristidis a été recueillie dans le Danube, à Giưrgewo Valachie), à Belgrade (Serbie), dans le Lom, à Rustschuck, (Bulgarie); ainsi que dans la Save près d'Agram, en Croatie. (Letourneux.)

\section{Anodonta Ramburi.}

Anodonta Ramburi, Mabille, in litt.

Cette espèce, d'une forme plus allongée que la précédente, à bord supéricur rectiligne, à rostre subtroncatulé et plus inférieur, à sommets plus ventrus, possède des valves épaisses, assez pesantes, n'offrant qu'un seul entre-bâillement médiocre entre l'angle et le rostre.

Long. $\max \ldots \ldots \ldots \ldots \ldots \ldots \ldots \ldots$ mill.

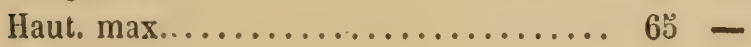


Épaiss، max. (à 29 des sommets, à 62 du rostre, à $48 \mathrm{du}$ bord antér., à 37 de l'angle postéro-dorsal et de la base de la perpendiculaire).............. 37 mill.

Long. de la crête ligam.-dors., des sommets à l'angle postéro-dorsal....... 42 -

Dist. de cet angle au rostre.......... 50 -

Corde apico-rostrale.............. 85 -

Hauteur de la perpendiculaire...... . $64-$

Distance de celte perpend. au bord antér.. 38 -

- du même point de cette perpend.

au rostre............... 71 -

- enfin, de la base de la perpend. à l'angle postéro-dorsal............ 68 -

Bord supéricur très long, rectiligne jusqu'à l'angle, puis brusquement descendant sur le rostre dans une direction faiblement cuncave. Région antérieure bien sphérique, faiblement décurrente à la base. Bord inférieur rígulièrement arqué. Région postérieure n'égalant pas deux fois l'étendue de l'antérieure, augmentant à peine jusqu'à 22 mill. en arrière de la perpendiculaire, puis s'atténuant à partir de l'angle, en une partie rostrale inféricure subtroncatulée.

Sommets ass $\bumpeq Z$ ventrus, légèrement proéminents, sillonnés par de trìs fines rides concentriques tremblo:ées. Crochets émoussés. Arête un tant soit peu cintrée, confondue dans la convexité, accusée seulement par un coloris plus foncé. Crête peu développée, légèrement comprimée vers l'angle.

Stries régulières, assez fortes, plus saillantes vers les contours, un peu feuilletées vers la partie postérieure. 
Epiderme brillant, d'une teinte verdâtre jaunacée, faiblement cendrée sur la région ombonale, passant au vert noirâtre sur la partie comprise entre l'arête et la crête. Intérieur d'une nacre blanche mate.

Ligaments : antéro-interne puissant, très antérieur, feuilleté, occupant toute la cardinale; postérieur robuste, saillant et symphynoté. Charnière latérale assez épaisse. Impressions en forme de palette.

Cette Anodonte habite la Nonette, à Chaalis près d'Elmenonville, dans l'Oise. (Mabille.)

\section{Anodonta Spiridionis.}

Anodonta Spiridionis, Lelourneux, in litt.

Cette espèce, obtuséinent subtriangulaire, est caractérisće par une région postérieure écourtée, se dilatant beaucoup en hauteur par suite de la forte convexité du bord palléal; par une partie rostrale, inférieure, excessivement obtusearrondie; par des valves minces, brillantes, pourvues de deux forts entre-bâillements, dont l'un postérieur s'étend entre l'angle et le rostre, sur toute l'étendue des contours antérieur et palléal.

Long. $\max . . . \ldots \ldots \ldots \ldots \ldots \ldots . . . .69$ mill.

Haut. $\max . . . \ldots \ldots \ldots \ldots \ldots . .61 \quad 61$ -

Epaiss. max. (à 22 des sommets, à 51 du rostre, à $46 \mathrm{du}$ bord antér., à 27 de l'angle postéro-dorsal, à 40 de la base de la perpend.).............. $36 \quad$

Long. de la crête ligam.-dors., des sommets à l'angle postéro-dorsal ...... 34 - 
Dist. de cet angle au rostre.......... 50 mill:

Corde apico-rostrale............. 74 -

Haut. de la perpendiculaire......... 37 -

Dist. de la perpend. au bord antér.... 35 -

- du même point de la perpend. au rostre.................. 61 -

- enfin, de la base de la perpend. à l’angle postéro-dorsal........ 63 -

D'après ces mesures, on remarque que le maximum de la convexité, rapproché des sommets et du bord supérieur, est presque à égale distance (à 5 mill. près) des extrémités antérieure et postérieure.

Bord supérieur allongé, bien rectiligne jusqu'à l’angle, puis descendant subitement sur le rostre par un contour droit, un tant soit peu concave. Région antérieure bien développée, légèrement anguleuse à son contour antérosupérieur, puis bien arrondie, enfin, décurrente à la base. Bord inférieur arqué-descendant jusqu'à 27 mill. en arrière de la perpendiculaire, ensuite remontant par une courbe bien arrondie vers la partie rostrale. Région postérieure écourtée, un peu plus d'une fois et demie plus longue que l'antérieure, augmentant en hauteur presque jusqu'au niveau de l'extrémité du ligament, puis à partir de l'angle s'atténuant brusquement pour se terminer par une large partie rostrale inférieure, arrondie, très obtuse et un tant soit peu ascendante.

Sommets convexes, non ventrus ni proéminents, couverts de très petites rides concentriques légèrement tremblotées. Crochets très aigus. Arête un tant soit peu cintrée, peu accusée, offrant, cependant, à son extrémité supérieure une trace de sillon. Crête comprimée bien développée. 
Stries régulières, çà et là assez saillantes, feuilletées vers la circonférence et notamment sur toute la region entre l'arète et la crête. Epiderme brillant, d'une teinte verdâtre radiée, passant au jaune vers le milieu des valves et au cendré sur les sommets. Intérieur d'une nacre bien irisée blanchâtre avec quelque reflets bleuacés.

Ligaments : antéro-interne filiforme, très antérieur; postérieur médiocre, tout à fait symphynoté. Lunule très allongée. Impressions spathuliformes.

La Spiridionis vit dans les marais de la Drave à Esseg, en Slavonie, et dans la Save, près de Belgrade, en Serbie. (Letourneux.)

\section{Anodonta Brusinge.}

Anodonta Brusinæ, Letourneux, in litt.

Cet Acéphale diffère du précédent par sa taille moindre, relativement moins haute et proportionnellement plus allongée; par sa région postérieure non écourtée, puisqu'elle dépasse deux fois l'antérieure, tandis que celle de la Spiridionis atteint seulement un peu plus d'une fois et demie; par sa partie rostrale allongée en forme de bec assez aigu-arrondi; par son arête plus cintrée; par son bord inférieur régulièrement convexe (chez la Spiridionis, ce contour d'abord convexe-descendant, offre, à 27 mill. en arrière de la perpendiculaire, une convexité plus accen. tuée); par son bord supérieur rectiligne seulement à partir des sommets (chez la Spiridionis, la direction rectiligne commence à 21 mill. en avant des sommets); par son angle postéro-dorsal plus saillant et nettement coupé à angle a.4. 1881. 
droit; par son contour intermédiaire entre l'angle et le rostre offrant une courbe concave très prononcée, etc.

La Brusinœ, sans compter un médiocre entre-bâillement entre l'angle et le rostre, en possède un autre considérable qui se développe dans toute l'étendue des contours antérieur et palléal.

Long. $\max . . . \ldots \ldots \ldots \ldots \ldots \ldots \ldots . .3$ mill.

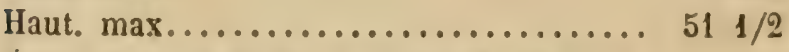

Épais. max. (à 27 des sommets, à 42 du rostre et du bord antér., à 30 de l'angle postéro.dorsal, à 27 de la base de la perpend.)......................

Long. de la crête ligam.-dors., des sommets

à l'angle postéro-dorsal............. 35 -

Distance de cet angle au rostre........ 43 -

Corde apico-rostrale.............. 67 -

Haut. de la perpendiculaire.......... 46 -

Dist. de cette perpend. au bord antér.... 26 -

- du même point de cette perpend. au rostre................... 58 -

- enfin, de la base de la perpend. à l'angle postéro-dorsal............ 57 -

Chez cette Anodonte, la convexité maximum se trouve juste à égale distance des extrémités antérieure et postérieure; la crête est plus longue (3ö mill.) que celle de la Spiridionis, espèce d'une plus forte taille; il résulte de ce caractère que les sommets de la Brusine sont plus antérieurs que ceux de la Spiridionis.

Bord supérieur bien rectiligne à partir des sommets jusqu'à l'angle, puis descendant à pic par un contour, 
d'abord vertical, qui se concave ensuite, pour s'arquer vers le rostre. Région antérieure médiocre, arrondie, décurrente à la base. Bord inférieur régulièrement convexe dans toute son étendue. Région postérieure allongée dans une direclion descendante, plus de deux fois plus longue que l'antérieure, augmentant jusqu'au niveau de l'extrémité du ligament, puis s'atténuant brusquement et se terminant par une partie rostrale, inférieure, arrondie et assez exiguë.

Summets peu convexes, comme comprimés, sillonnés par de belles rides ondulées. Crochets très aigus. Arête légèrement cintrée, nettement accusée par deux radiations vertes divergentes. Crête développée, très comprimée vers l'angle postéro-dorsal, qui est coupé à angle droit.

Stries régulières, peu saillantes, très faiblement feuilletées postérieurement. Epiderme brillant, d'un jaune verdâtre plus foncé vers la région de la crête, et passant à une teinte rouge-brique vers les sommets. Intérieur d'une belle nacre irisée bleuâtre.

Ligaments : antéro-interne filiforme, très antérieur; pos. térieur très allongé, entièrement symphynoté. Lunule remarquablement allongée (12 mill.).

Cette espèce, ainsi que la précédente, dédiée par notre ami le conseiller Letourneux, au professeur Spiridion Brusina d'Agram, habite dans la rivière de KrapinaTœplitz, en Croatic, et dans la Save, près d'Agram. (Letourneux.) 


\section{Anodonta Plctetiana.}

Anodonta Pictetiana (pars), Mortillet in : Brot, Nayades

Léman, p. 45, pl. VIIl, f. 1 (seulement), 1867.

J'ai énoncé, pages 102 et 103, mon opinion sur cette Anodonte.

J'ai dit que l'suteur de la Pictetiana 2vait amalgamé sous cette appellation, des cygnœa, des cellensis, des ana tina, des tumida, etc.

J'ai émis l'avis que M. Brot, de Genève, après avoir éliminé ue cetle espèce une forme cygnœa var. rostrata, avait réuni sous le même nom de Pictetiana, la tumida du groupe des Brotiana, la lacuum du groupe Glyciana, enfin, qu'il avait fait figurer, à la planche VIII (fig. 1) de ses nayades du Léman, Ia vraie Pictetiana.

Cet Acéphale est très birn représenté. Cette représenta. tion suffit amplement à la connaissance de cette furme.

La Pictetiana habite à l'extrémité du lac de Genève, près de Villeneuve. II: G. Coutagne a recueilli dans le lac de Neuchâtel, près de la ville du même no:n, des échantillons identiques à ceux de Villeneuve.

\section{Anodonta exocha.}

Je rapporte à cetle espèce le Mytilus cygnœus représenté dans les Flussconchylien de Schröter à la planche III, fig. 1 . Cette figure rend assez bien le port et l'aspect de l'exocha.

L'exocha est une Anodonte ovalaire presque aussi haute 
en avant qu'en arrière, à région postérieure écourtée, terminée par un petit rostre camard. La convexité, chez cet Acéphale, se trouve plus reportée en arrière qu'en avant. D'après la mensuration de plusieurs échantillons, le maximum, un peu plus rapproché du rostre que du bord antérieur, est presque à égale distance des sommets, de l'angle et de la perpendiculaire. Chez toutes les autres Piscinales que je connais, le maximum d'épaisseur esı gé. néralement plus ou moins antérieur, avec une tendance à se porter vers la région ombonale.

Les valves sont fortement entre-bâillées en arrière, entre l'angle et le rostre, et en avant dans toute l'étendue des contours antérieur et palléal.

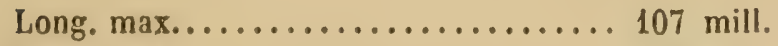

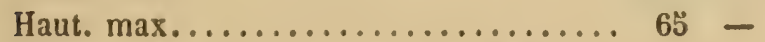

Épaiss. max. (à 32 des sommets, à 53 du rostre, à 54 du bord antér., à $351 / 2$ de l'angle postéro-dorsal, à 36 de la base de la perpend.).................

Long. de la crête ligamento-dorsale, des sommets à l'angle postéro-dorsal..... 38 -

Dist. de cet angle au rostre.......... 47 -

Corde apico-rostrale.............. 78 -

Haut. de la perpendiculaire.......... 63 -

Dist. de cette perpend. au bord antérieur. 11 . -

- du même point de cette perpend. au rostre...................6. 66 -

— enfin,de la base de la perpend. à l'angle postéro-dorsal.............. 69 -

Bord supérieur presque rectiligne ou un tant. soit peu ar- 
quéjusqu'å l'angle, puis descendant obliquement et présen. tant une légère concavité avant d'arriver au rostre. Région antérieure grande, amplement développée en hauteur, à contour exactement sphérique. Bord inférieur régulièrement arqué, remontant sur le rostre par un contour bien arrondi. Région postérieure écourtée, une fois et demie plus longue que l'antérieure, conservant sa grande hauteur, qui augmente peu jusqu'au niveau de l'extrémité du ligament, puis s'atténuant en un rostre écourté, presque médian, très obtus, arrondi, ressemblant à un rostre camard.

Sommets convexes, néanmoins comme comprimés, sillonnés par des rides ondulées ellipsoïdes. Arête cintréeconcave, accusée par deux radiations vertes divergentes, et notamment, par un sillon formant dépression, sillon qui se fait sentir sur la nacre intérieure sous l'apparence d'une zonule crispulée. Crête dorsale peu développée, assez comprimée.

Stries irrégulières, çà et là saillantes, fort peu feuilletées vers la circonférence. Ces stries sont, à un degré plus ou moins prononcé, suivant les échantillons, interrompues par de très fines striations creuses, rayonnantes, qui donnent à la surface une apparence treillissée. Ces striations rayounantes ne descendent pas jusqu'à la circonférence.

Épiderme d'un jaune bleuâtre s'éclaircissant en cendré sur la région ombonale, pour passer au rougeàtre sur les sommets. Sur toute la surface épidermique, on remarque de très fines radiations brunes, et seulement deux à quatre radiations bien vertes sur la région de l'arête. Intérieur d'une nacre blanche irisée.

Ligaments : antéro-interne, filiforme, très antérieur; postérieur complètement symphynoté. Lunule très longue. 
L'exocha habite dans les vieux relais du Rhône, audessous d'Arles. D'après Schröter cette espèce vit également dans les eaux profondes de la Bavière.

\section{MULIERANA}

Les Milletiana sont des Anodontes de forme presque cir culaire (sauf la subrhombea, qui est un peu ovalaire, et la Dantessantyi, qui est plutôt subquadrangulaire), par conséquent fort peu allongée et très dilatée dans le sens de la hauteur. Ces espèces vivent indifféremment dans les lacs, les étangs ou les canaux aux eaux profondes et vaseuses.

\section{Anodonta subcircularis :}

Anodonta subcircularis, Clessin, Anod. in : $2^{\circ}$ édit. Chemnitz, p. 87, pl. XXII, f. 3-4, 1876.

Belle espèce du lac de Scutari, au nord de l'Albanie.

Clessin rapporte à cet Acéphale l'A nodonta lata 1 de Parreyss, mss. Je ne puis exprimer d'opinion sur ce rapprochement, que je signale à titre de rensei rnement, attendu que je ne conna is pas cette lata, qui n'a jamais été décrite.

1. Non Anod. lata de Rafinesque, espèce américaine. 


\section{Anodonta Interrogationis.}

Anodonta Kickxii, var. interrogationis, Colbeau, Mol'. Belg. in: ann. soc. malac. Belg. III, 1868, p. 107, pl. III, f. 2.

Superbe Anodonte recueillie dans l'étang (actuellement desséché) d'Etterbeek, en Belgique.

\section{Anodonta milleti.}

Anodonta Milleti, Ray et Drouët, desc. Anod. in : Rev. zool., p. 255, pl. I, f. 1, 1848; et Dupuy, Moll. France (6॰ fasc., 1852), p. 617, pl. XXI, f. 16.

Anodonte très caractérisée et bien rendue sur les planches que je viens de citer.

Elle a été découverte dans un réservoir très profond alimenté par la Rance, à Montabert, à 12 kilum. de Troyes (Aube). Je l'ai reçue parfaitement typique, sous l'appellation fautive de sulponderosa, des environs d'Arles (Bouches. du Rhône), où elle vit dans les endroits les plus profonds des vieux relais du Rhơne, au-dessous de cette ville.

\section{Anodonta episema.}

Long $\max . . . \ldots \ldots \ldots \ldots \ldots \ldots \ldots . . .67$ mill.

Haut. max................ 73 -

Epaiss. max . (à 33 des sommets, à 55 du du rostre, à 50 du bord antér., et à 40 de l'angle postéro-dorsal et de la base de la perpend....$\ldots \ldots \ldots \ldots .31$. 
Long. de la crête ligamento-dorsale, des sommets à l'angle post.-dorsal. 38 mill.

Distance de cet angle au rostre...... 55 -

Corde apico-rostrale............ 82 -

Hauteur de la perpendiculaire...... 72 -

Dist. de la perpend. au bord antérieur. 43 -

- du même point de la perpend. au rostre................. $66 \quad$

- enfin, de la base de la perpend. à l'angle postéro-dorsal........ 73 -

Coq. subcirculaire dans un sens un peu allongé, présentant postérieurement deux parties très obtusément an. guleuses (à l'angle postéro-dorsal et au rostre) par suite de la direction rectiligne du bord entre l'angle et le rostre. Valves peu hombées, bien brillantes, d'un beau coloris, offrant deux bâillements : un inférieur (non antérieur) se prolongeant sur toute l'étendue du bord palléal; l'autre postérieur eutre l'angle et le rostre.

Bord supérieur rectiligne jusqu'à l'angle postéro-dorsal, puis descendant obliquement en ligne droite sur le rostre. Région antérieure très dilatée, bien arrondie, néanmoins un peu décurrente à la base. Bord inférieur bien convexe jusqu'au rostre. Région postérieure relativement courte, pas tout à fait une fois et demie plus longue que l'antérieure, augmentant peu en hauteur et se terminant brusquement par un contour rectiligne descendant obliquement sur une partie rostrale inférieure, obtuse et peu prononcée.

Sommets presque médians, écrasés, non convexes, très élégamment sillonnés de rides. Crochets proéminents, aigus comme des aiguilles. Arête dorsale confondue dans 
la convexité générale, qui est bien régulière, ne s'accusant que par deux ou truis radiations divergentes d'un très beau vert. Crête assez développée, normalement comprimée.

Stries fines, régulières, plus fortes et saillantes vers les contours. Epiderme bien brillant, d'un beau coloris jaunâtre passant au cendrés sur la région postéro-ventrale, au rouge-brique sur les sommets; enfin, au brun-olivâtre vers la crête, et orné, en outre, de très fines radiations accusées seulement vers les contours. Intérieur d'une belle nacre blanche irisée.

Ligaments : antéro:interne médiocre, filiforme; postérieur entièrement recouvert. Lunule très allongée.

L'episema vit dans les vieux relais du Rhône, au-dessous d'Arles, dans les endroits vaseux et très profonds.

\section{Anodonta subrhombea.}

Anodonta subrhombea, Brown, illust. Conch., p. 80 ,

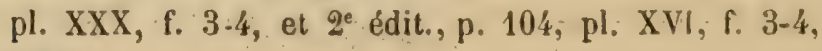
1845.

Je rapporte à cette espèce l'anodonta piscinalis de M. Drouët, très bien représentée, du reste (pl. V, f. 1), dans ses études sur les Naïades de France.

Cet Acéphale a été recueilli en Angleterre, dans l'Irwell près de Manchester, dans un cours d'eau du vallon Dintingr près Glossop; en France, elle a été récoltée aux environs de Villemereuil près de Troyes (Aube). 


\section{Anodonta Dantesmantyi.}

Anodonta Dantessantyi, Ray, in litt.

Cette Anodonte, que le sieur Drouët a confondue également avec sa piscinalis, vit dans le Villegusien, près de Langres (Haute-Marne).

Elle a été bien figurée sous le nom d'anodonta piscinalis, variété (avec un point de doute), par Rossmässler, dans son Iconographie (fig. 416), où elle est signalée du Weser près de Brême. Cette figure suffit à la connaissance de cette espèce, dédiée par J. Ray, à l'abbé Dantessanty, de Troyes.

\section{Anodonta elachista.}

Anodonta elachista, Bourguignat, in : moll. nouv. $\left(6^{\circ}\right.$ décade, 1866), p. 197, pl. XXXI, f. 12-14.

Très jolie petite forme presque circulaire, assez abondante dans le canal de Bouc, à Arles (Bouches-duRhône).

L'elachista est la dernière espèce vivante d'Europe que j'ai à signaler.

A cette liste, je crois qu'il convient d'y joindre cette autre relative aux Anodontes fossiles; mais, comme ces 


\section{$-364-$}

fossiles sortent de mon cadre, je ne ferai que les indiquer aussi succinctement que possible, d'autant plus que presque toutes sont douteuses. Elles ont, en effet, été décrites, signalées ou figurées, tantôt d'une façon si superficielle, tantôt d'une manière si imparfaite, soit d'après un fragment, soit d'après une simple empreinte, qu'il est très difficile de savoir ce qu'elles peuvent étre. Anodonta anatinoides, Klein, in: Wurt. Jahrb. 11, 1846, p. 92. - Ziéten pense que c'est un Unio.

- antiqua, Ch. d'Orbigny, in : mag. zool., 1836, pl. LXXVIII, f. 2. - Des argiles plastiques de Meudon, près Paris. - Cette espèce est une des rares Anodontes fossiles bien caractérisées. Elle appartient au groupe de l'Acalliana.

- aquensis, Matheron, cat. foss. corps org. Bouches-duRhône, p. 171, pl. XXIV, flg. 9, 1842. - Des gypses de Beaulieu, près d'Aix, en Provence. - Petite forme indécise (long. 29, haut. 17 mill.), peut-être non adulte, représentée d'après une empreinte.

- Bronni, d'Ancona. - Du pliocène supérieur de la vallée de l'Arno (Italie).

- Cordieri, C. d'Orbigny, in : mag. zool., 1836, pl. LXXVIII, f. 1. - Des argiles plastiques de Meudon, près Paris. Espèce, de forme solénoïde, regardée par les uns comme une Mutela (Iridina), par les autres comme un Unio. D'après un moule trouvé par M. le professeur Hébert de la Sorbonne, la charnière de la Cordieri serait denticulée (voir ce que dit notre regretté ami Deshayes (an. s. vert. hass. Paris, I, 1860, p. 801), au sujet de cette soi-disant Anodonte.

- Fardanensis, Matheron, Rech. comp. dép. fluv. lac., 1862, et, d'Archiac, Paléont., France, p. \$18, 1868. - 
Des lignites de Fuveau et de Gardanne, en Provence.

- Heeri, de C. Mayer, de la mollasse supérieure d'eau douce de Suisse, à Schrotzburg et Spreitenbach.

- Lavateri de Brongniart. Espèce d'Unio, d'après Bronn (ind. Paléont., 1848).

- prœdemissa, Ludwig, du miocène inférieur d'eau douce de la vallée du Khône.

- viridis, Ludwig, in : Paléont. VIIl, p. 196. pl. LXXII, f. 8-10. - Du pliocène lacustre supérieur du centre de l'Allemagne.

- 1 tener et tenuissima, Eichwald, urwelt Russlands, etc., I, 1840 , p. 100 et 101, pl. IV, f. 1 et 2. - Ces espèces paraissent être des Unios.

En somme, presque toutes cés Anodontes sont douteuses, toutes sont tertiaires. On n'en connait pas des étages antérieurs, bien qu'on en ait cité une, plus que problématique, l'Anodonta Liaso-Keuperina de Braun, d'une couche lacustre des environs de Bayreuth en Bavière, couche considérée comme contemporaine des dépôts du Lias.

Quelques esprits timorés pourront être effrayés, peutétre, du grand nombre d'Anodontes que je viens de signaler. On est loin, cependant, de connaitre toutes celles qui existent.

Il y a, en effet, de vastes contrées inexplorées en Europe, telles que la Slavonie, la Serbie, la Bosnie, la Bulgarie, la Roumélie, etc., où toutes les formes sont nou-

1. On trouve cité encore par Neumayr et Paul (Cong. et Palud. Sláv. 1875), une Anudunta lenuis dés tertiaires lacustres du bassia du lac de Constance. 
velles. Dans les régions même les mieux explorées, comme la France, la Suisse, l'Allemagne centrale, on en découvre chaque jour d'inconnues. En présence de tels faits, je reste convaincu que, dans une vinglaine d'années, cette liste sera doublée, peut-être triplée. Cet accroissement inévitable d'espèces me parait d'autant plus évident, que même actuellement il en existe beaucoup d'autres que j'ai passé sous silence, parce qu'il m'a été impossible de contrôler leur valeur.

Ces espèces, malheureusement inédites, sont les suivantes :

Anodonta arcuata Ferrussac, art. Anod, in : dict. class. d'hist. nat. I, 1822, p. 397 (sans desc.). - Des lacs de la haute Autriche et de l'Oder près de Stettin.

- assimilis, Ziegler, mss. in mus. Vindob. - D'Autriche, citée par Gallenstein Karut. conch., p. 22, 1848, et p. 66, 1852.

- atrovirens, Shuttleworth, mss, citée par Poetel (cat. conch. samml., p. 147, 1873). - De Mosano (Suisse):

- Borysthenica, Krynicki, in : Bull. natur. Moscou, 1837, p. 58 (s. desc.). - Du sud de la Russie.

- canescens, Stentz, mss. - De Dalmatie. Villa (disp. syst.) conch., p. 40, 1840, et, in: Bull. malac. ital., 1871, p. 93) regarde cette Anodonte comme une variété minor de la Limpida.

- collaris, Parreyss, mss. - De Bavière, citée par Anton (verz. conch., p. 16, 1839).

- compressa 1 Parreyss, mss. - De Dalmatie, mentionnée par Brusina (malac. Croat., p. 32, 1870).

1. Non compressa de Menke (Syn., 20 édit., p. 106, 1830). Eispece de pseudanodontes. 
- confervigera, Schlütter, inss. - De Carniole et de Carynthie. Tous les auteurs qui citent cette espèce, la rapportent à la rostrata.

- corrosa, Parreyss, mss. - D'Autriche.

- corrosa, Kutschig, mss. in: Brusina, mal. Croat., p. 32, 1870. - De Croatie. Cette Corrosa de Kutschig, est-elle semblable à celle de Parreyss? C'est ce que j'ignore.

- crassa, Fitzinger, Oesterr. weichth., p. 120 (s. desc.), 1833. - De l'Autriche. Quelques auteurs croient voir en elle la ponderosa.

- croatica, Kutschig, mss., in : Brusina, mal. Croat., p. 32, 1870. - De Croatie.

- cuneata, Küster, mss., mentionnée par Drouët. D'Autriche.

- Dalmatina, Parreyss, mss. - Cette espèce signalée par quelques'auteurs est peut-être la même que la Dalmatina de Kutschig, forme que l'on rapporte à la limpida de la Narenta; en Dalmatie.

- Dentata. - Mytilus dentatus, Turton, conch. dict. p. 115, 1822. - D'Angleterre. Lov. Reeve (moll. Brit., p. 216, 1863) range cette forme parmi les synonymes de la cygnœea. Gray (man, shells Brit., p. 200, 1840), dit que cet Acéphale est une variété anormale de la cygnœa.

- dentiens, Menke, mss. - D'Autriche, citée par Rossmässler, Iconogr. V et VI, p. 23, 1837.

- deplanata, Ziegler, mss. in mus. Vindob. - D'Autriche, signalée par Gallenstein. Karnt. conch., p. 22, 1848 et p. 68, 1852 .

- derasa, Ziegler, mss. in mus. Vindob. - D'Autriche.

- excentrica, Ziegler, mss. - De Carniole, mentionnée par Villa, disp. syst. conch., p. 40, 1840, ct par Schmidt 
(conch. Krain, p. 27, 1847), qui la rapporte \& sa Cygnœa

- fluviatilis, Dillwyn. - Du Caucase russe, citée par Middendorff (Mull. Sibir., p. 402, 1851).

- fragilis (non Lamarck), Fitzinger, OEsterr., p. 120 (s. dessc.), 1833. - Du Danube et des cours d'eau de l'Autriche.

- fucata (Mytilus fucatus de Dillwyn), Ferussac, art. Anod., in : Dict. class. d'hist. nat. I, 1822, p. 397. De l'Angleterre et de France dans la Bresse, et les étangs de Saint-Etienne de Montluc, dans la LoireInférieure.

- fuscata Ziegler, mss. in mus. Vindob. - D'Autriche, citée par Gallenstein (Karnt. conch., p. 22, 1848, et p. 67, 1852). - En 1856 (Amén. malac. I, p. 154), j’ai signalé cet Acéphale du Monténégro, d'après l'avis du sieur Drouët. Je suis certain, à présent, que cet auteur m'a donné une fausse indication. Il est vrai qu'à cette époque,j'étais assez bête pour croire à ses bonnes déterminations.

- gibba, Kutschig, mss. (non, gibba de Held). Cette espèce a été rapportée à tort à l'exulcerata de Villa par Clessin (Anod., par 127, 1876), exulcerata qui, entre parenthèses, n'est pas celle de Villa.

- glabra, Ziegler, mss. in : Anton, verz. conch., p. 16, 1839. Du Veldeser-see. Cette glabra doit être différente de celle de Villa, dont j'ai donné ci-dessus la description.

- grossa, Parreyss, mss. - D'Autriche, mentionnée par Gallenstein (Karnt. conch., p. 22, 1848, et p. 66, 1852).

- latissima, Kokeil, in : Anton, verz. conch., p. 16, 1839. 
- De Klagenfurth. Quelques auteurs rapportent cette forme à la rostrata.

- lingua, Yoldi, mss. in : Mörch, cat. Yoldi, p. 609, et, Syn. moll. Dan., p. 83, 1864. - Du Dảnemark.

- lyngbiana, Mörch, Syn. moll. Dan., p. 83, 1864. - Du Danemark.

- minuta, Parreyss, mss. in: Villa (Bull. malac. ital., 1871, p. 94). - De Lombardie.

- nitida, Ziegler, mss. in mus. Vindob. - D'Autriche.

- obvoluta, Menke, mss. - D'Autriche.

- paludosa Gray (Anodon paludosus, Turton, Biv., p. 240 , pl. XV, f. 6, 1830). Gray (Man. shells Brit., p. 240, 1840), rapporte cette forme à $s a$ Cygnæa.

- Parreyssi, Kinberg, citée par M. Drouët. - De la Suède.

- plalyrhyncha, Kokeil, in : Gallenstein, Karnt. Conch., p. 22, 1848, et p. 69, 1852. - De Carniole.

- polymorpha, Kutschig, mss. in: Brusina, malac. Croat., p. 32, 1870. - De Groatie.

- polyrhynchus, Kokeil, signalée par Kreglinger, Binn. Moll., p. 333, 1870.

- proboscidæa, Ziegler, mss. in mus. Vindob. - D'Autriche.

- prolongata, Ziegler, mss. in mus. Vindob. - D'Autriche -Quelques auteurs regardent cette forme comme une variété de la rostrata.

- recurvirostra, Kuster, mss. D'Autriche. Ainsi que la précédente, cette forme est assimilée à la rostrata.

- riparia, Pfeiffer. - Du Volga. Espèce mentionnée par Eichwald (Bull. nat. Moscou, 1838, p. 163, et fawn. Casp. Gaus., p. 264, 1838).

- Rossmässleri, Kinberg. - De Suède. - Citée par Drutuët, 2A1. 1881. 
1854 (naiad. France, 7• art., p. 17). - Non Anod. Cygnea, var. Rossmässleri, de Malzine, 1867 (Malac. Belg., p. 32), variété créée pour la figure 67 de Rossmässler, figure qui représente le type de mon Anodonta eucypha. - Si les noms de Rossmassleri, de Kinberg in: Drouët, 180ّ4, Rossmassleriana, Dupuy, $18 \div 3$ et 185̋2, n'avaient pas été antérieurs à celui établi par Malzine en 1867, je n'aurais pas créé le nouveau nom d'eucypha.

- Sabljarii, Kutschig, mss. De Croatie. Teste Brusina, mal. Croat., p. 32, 1870.

- sinuosa (non Lamarck), Mauduyt, tabl. moll. Vienne, p. 15, 1839. De la Vône à Sauxay et des étangs de l'ar'rondissement de Civray (Vienne). D'après l'abbé Dupuy (Hist. moll., p. 604), cette espèce serait une cellensis, ou pour mieux dire une cygnæa.

- spreta, Ziegler, mss. - D'Autriche. - J'ai autrefois, sur une fausse indication de M. Drouët, signalé cette forme comme une variété de l'oblonga, en Bulgarie et en Roumélie (voir Amén. malac., I, p. 153, 1856).

- spuria, Yoldi, mss. Du Danemark. Ĺspèce rapportée à turt à mon Helvetica de Suisse, par Mörch (Syn. moll. Dan., p. 87, 1864).

- subalata, Ziegler, mss. in mus. Vind. D'Autriche.

- subdepressa, Parreyss, mss. - D'Autriche.

- sulcata (non Lamarck), Nilsson, moll. Suec., p. 113 et 114, 1822. De Suède. Cette espèce a encore été signalée par Kleeberg en Allemagne.

- stenorhyncha, Kuster, mss. D’Autriche.

- triangularis, Vidovic, mss. - De Dalmatie. Mentionnée par Frauenfeld, faun. Dalm., p. 76, 1856.

- triangulata, Parreyss, mss. De la Kerka en Dalmatie. Citée par Brusina, Mal. Croat., p. 131, 1870. Ce même 


\section{$-371-$}

auteur (Moll. Dalm., p. 131, 1866) mentionne également de la Kerka une Anodonta triangulata de Kutschig, mss. Quid?

- tumida (non Kuster), Fitzinger, OEsterr. weichth, p. 121 (s. desc.), 1833. - Du Danube, près Petronell (Autriche).

- vetula, Ziegler, mss. in mus. Vindob. - D'Autriche, citée par Gallenstein, Karnt. conch., p. 22, 1848, et p. 68 1852.

- virens, Stentz, mss. - De Croatie. Mentionnée par Villa, Disp. syst. conch., p. 40, 1840 .

Etc., etc.

Toutes ces Anodontes (je suis sûr que j'en oublie) sont des formes inédites qui me sont inconnues. J'ai tenu, néanmoins, à les faire connaître, à titre de renseignement, pour que les malacologistes futurs, plus heureux que moi dans leurs recherches, ne les laissent pas tomber dans l'oubli ; car il doit y avoir incontestablement, parmi elles, des formes nouvelles et intéressantes.

Il me reste maintenant à dire quelques mots sur quatre espèces publiées sous les noms d'Anodonta contorta, dorsuosa, cymbalica et Mœsica.

1. Anodonta (anodon) contorta, Brown, lllust. conch., p. 81 , pl. XXX, f. 7.8, et, $2^{\bullet}$ édit., p. 106, pl. XVIII, f. 1-2, 1845.

Cette espèce est vraisemblablement la plus singuliêre des Anodontes; elle est caractérisée par des valves iné- 
gales, l'une, la sénestre (d'après la gravure est plus forte, plus ventrue que la dextre. Si ce caractère n'était pas le résultat, à mon sens, d'un état morbide, il serait suffisant pour élever cette espèce au rang gẻnérique, malheureusement, je le crois anormal.

Cette contorta a été recueillie par sir Thomas Gloves, esq., au nombre de 30 échantillons, dans les fossés d'une prairie marécageuse, non loin de la maison d'école de Repton, prìs de Burton, sur le Trent. Depuis cette découverte, qui remonte à 1840 , cette localité a été détruite par suite des énormes remblais exécutés à l'occasion de la construction d'un pont sur cette rivière.

$2^{\circ}$ Anodonta dorsuosa, Drouët, union. nouv. in : Journ. Conch., janvier 1881, p. 30.

Cette dorsuosa, signalée de la Saône à Pontailler et à Charrey (Côte-d'Or), est une Pseudanodonta du groupe de l'elongata.

M. Georges Coutagne a recueilli cette même forme également dans la Saône à Auxonne (Côte- d'Or), où a elle forme une colonie très lucaliscé à quelques mètres en amont du grand barrage de la Saône, sur un fond de gravier mêlé d'un peu de vase.

" Elle abonde, ajoute M. Coutagne, principalement près de la rive gauche en aval de la petite île couverte de saules qui parlage la rivière en deux bras à peu près égaux.

$3^{\circ}$ Anodonta cymbalica, Drouët, in : Journ. Gonch., 1879, ..p. 333. - Du lac de Scutari, dans le nord de l'Albanie. 
$4^{\circ}$ Anodonta Mœsica, Drouèt, in Journ. Conch., 1881, p. $29 \longrightarrow$ De la Save à Usce, en Serbie.

Ces deux espèces pourraient bien appartenir au groupe des Briandiana. Je dis : « pourratent bien », parce qu'elles sont si mal comprises et surtout si faussement décrites, qu'il y aurait de la témérité à formuler une affirmation.

Que penser d'un auteur dont la prétention est de connaitre les Acéphales mieux que personne, qui, au bout de 30 ans d'études sur ces animaux, en est arrivé à ne pas savoir ce qui est concave ou convexe. On pourrait croire, en lisant ces lignes, que je m'amuse à inventer une mauvaise plaisanterie. Eh bien, non ! je cite : «Anod. cymbalica, - ... antice late rotundata, postice breviter truncato. subrotundata, infra concaviuscula. 》 - « Anod. moesica, - ... antice attenuato-rotundata, postice medio dilatata, infra perconcava, - Anodonta gravida. - ... antice late rotundata, infra concava, etc. $D$

Et ainsi de même chez toutes les autres descriptions.

Ce qu'il y a d'étonnant dans ces descriptions, c'est que les parties antérieure et postérieure sont reconnues arrondies, par conséquent convexes, tandis que cette pauvre partie inférieure, qui est bien réellement arquée, attrape la désignation de concave (concava) ou même de très concave (perconcava).

Pourquoi celte différence d'appréciation entre les différents bords des Anodontes, lorsque ces Anodontes sont convexes dans toutes leurs parties? Pourquoi décrire faussement ce qui n'est pas? est-ce dans le but de rendre ses descriptions plus claires et plus compréhensibles que « ce père de la science 》 dénature à plaisir les caractères? Il me semble, cependant, que les Anodontes sont d'une 
étude déjà assez difficile, que les confusions sont suffisamment nombreuses pour qu'on ait le droit de n'aroir pas à se débattre encore avec des caractères faussement énoncẻs.

Je ne dirai plus que quelques mots.

Je n'ai pas voulu faire une monographie des Anodontes d'Europe. Mon intention a été seulement de donner un aperçu sur les différentes espèces européennes de ce genre.

J'ai groupé et j'ai distingué les formes stables que j'ai pu connaitre, formes qne je possède presque toutes dans ma collection.

Si j'ai éliminé certaines espèces, ou si j'ai élevé certaines autres au rang spécifique, j'ai agi, en ces circonstances, en conscience et sans parti pris.

J'ai changé la méthode d'envisager ces coquilles, parce que l'ancienne m'a parue mauvaise et ne répondant plus aux connaissances scientifiques actuelles.

Avec l'ancienne méthode, chacun était libre de donner à un Acéphale une position fantaisiste, suivant que l'on accordait une prédominence plus ou moins prononcée à l'angle pustéro-dorsal.

Avec la méthode nouvelle, plus d'arbitraire. Les sommets ont la prédominence sur tout le bord supérieur; ils acquièrent l'importance qu'ils ont réellement dans la nature; ils servent de point de départ à cette perpendiculaire, tirće dans l'axe du rayon visuel, par laquelle on obtient le partage d'une bivalve en deux régions : une antérieure, une postérieure.

Les convexités ont été prises au compas d'épaisseur, abstraction faite de la courbure des valves. 
Les distances entre le maximum de convexité et les divers points du contour ont été calculées, comme celles d'un plan. Si j'avais agi autrement, ces distances auraient pu être faussées, chez certaines espèces, par les courbes plus ou moins exagérées des valves.

J'ajouterai enfin, pour terminer, que ce travail, où j'ai tout changé, tout modifié : appréciation des formes, classification, groupement, description, mensuration, etc., restera incompris si l'on ne fait pas table rase des anciennes données, des vieilles idées qui ont cours en ce moment.

NotA. - Il vient de me parvenir, trop tard pour que je puisse m'en occuper, un nouveau mémoire de M. Drouët : a Unionidee de la Russie d'Europe, o où les espèces nouvelles (décrites toujours d'après cet intelligen $\ell$ système que l'on sait) sont les suivantes: Anodonta ostaria du Dnieper, parmata du sud de la Russie; Sieversi. Georgiana, $C_{y-}$ rea et Leukoranensis des provinces caucasiques. - J'ai reçu, du reste, depuis la publication (mai 1880) de mon chapitre sur les " Anodontes d'Asie ", un assez grand nombre de formes asiatiques nouvelles. Toutes ces espèces seront le sujet d'un mémoire spécial dans la suite de ces - satéraud *

Saint-Germain, mai 1881.

J. R. B. 



\title{
TABLE DES NOMS D'ESPÈCES
}

\author{
ET DES
}

\section{APPELLATIONS SYNONYMIQUES}

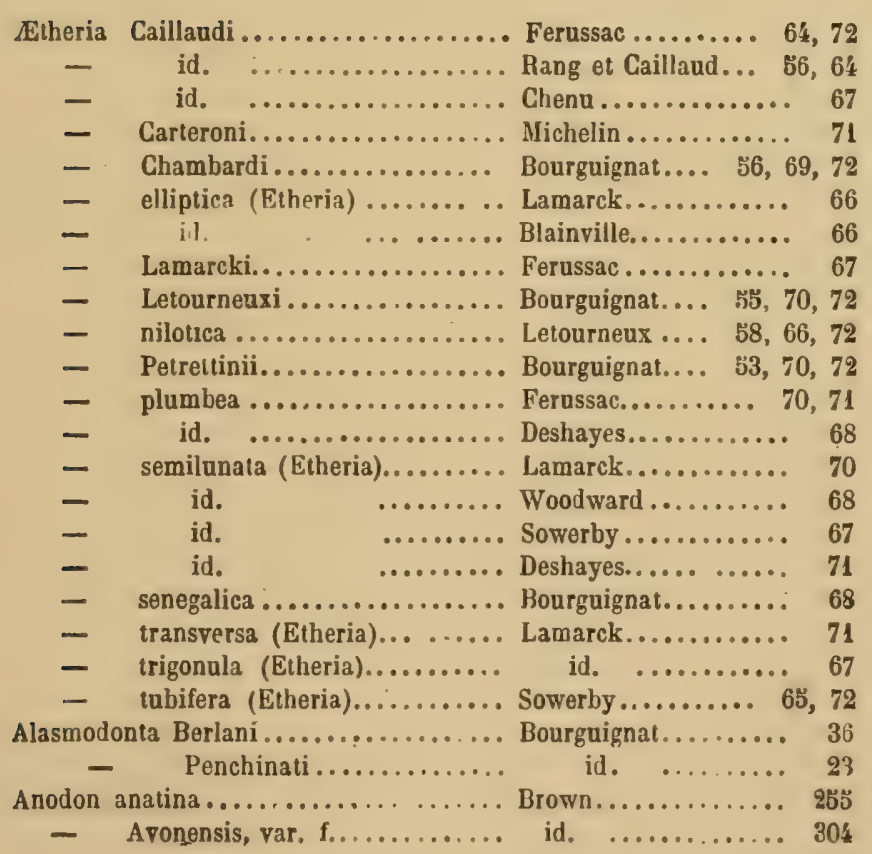




\begin{tabular}{|c|c|c|c|}
\hline Arodon & cellensis & Brown............. & 194 \\
\hline - & paludosus.................... & Turton............... & 369 \\
\hline - & ponderosa, var.............. & Brown.............. & 29 ! \\
\hline - & stagnalis ................... & id. $\ldots \ldots \ldots \ldots \ldots$ & 108 \\
\hline nodonta & abbreviata................... & Bourguignat.......... & 296 \\
\hline- & acallia $\ldots . . . \ldots \ldots \ldots \ldots \ldots$ & J. Ray............ & 276 \\
\hline - & acyrta...................... & Bourguignat.......... & 130 \\
\hline 一 & Adamii.................... & id. $\ldots . . . \cdots$. & 191 \\
\hline - & psis ................. & id. $\ldots \ldots \ldots \ldots$ & 47 \\
\hline - & is $\ldots . . . \ldots \ldots \ldots . . . . .$. & Charpentier.......... & วิ6 \\
\hline - & anatina................... & Rossmassler...... 253, & 250 \\
\hline - & id. $\quad \ldots \ldots \ldots \ldots \ldots \ldots \ldots$ & Draparnaud...... 25\%, & 334 \\
\hline - & id. $\ldots \ldots \ldots \ldots$ & Moquin-Tandon........ & 254 \\
\hline - & id. $\quad \ldots \ldots \ldots \ldots \ldots \ldots, \ldots$ & Dupuy.......... 25\%, & 283 \\
\hline - & id. $\quad \ldots \ldots \ldots \ldots \ldots, \ldots, \ldots$ & C. Pfeiffer............. & 205 \\
\hline- & td. $\quad \cdots \cdots \cdots, \ldots, \ldots, \ldots$ & Clessin......... 255, & 287 \\
\hline - & id. $\quad \ldots \ldots \ldots \ldots \ldots \ldots \ldots$ & Mousson ............. & 82 \\
\hline - & id. $\quad \ldots \ldots \ldots \ldots \ldots \ldots$ & Dronët............... & 266 \\
\hline - & id. var. abbreviata....... & Brot................. & 171 \\
\hline - & id. var. elongata....... & 1d. . . . . . . . . . . . & 171 \\
\hline - & id. monstrosa............ & id. . ................ & 296 \\
\hline - & id. tуріса............... & is. ................. & 296 \\
\hline - & anatinoides................ & . Klein.............. & 364 \\
\hline - & tris............. & $r \ldots \ldots \ldots \ldots$ & $14 !$ \\
\hline - & ................ & - Ch. d’Orbigny ........ & 354 \\
\hline 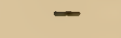 & la........ & . Bourguignat.......... & 164 \\
\hline - & A pollonica ................... & id. $\quad \ldots \ldots \ldots \ldots$ & 91 \\
\hline - & 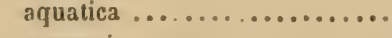 & . Servain .............. & 220 \\
\hline - & aquensis.....$\ldots \ldots \ldots \ldots$ & . Matheron:............ & 364 \\
\hline - & arcuata $\ldots \ldots \ldots \ldots \ldots \ldots \ldots$ & Ferussac ............. & 366 \\
\hline - & arealis....$\ldots \ldots \ldots \ldots \ldots \ldots$ & . Kuster............. & 283 \\
\hline - & Arelatensis............ & . Jacquemin ........... & 286 \\
\hline - & naria ................... & . Bourguignat.......... & 139 \\
\hline - & Aristidis .................. & ...... & 347 \\
\hline - & arvernica $\ldots . . . \ldots \ldots \ldots \ldots$ & ......... & 158 \\
\hline - & assimilis $\ldots \ldots \ldots \ldots \ldots \ldots$. & - Ziegler............ & 366 \\
\hline - & trovirens................... & - Shuttleworth ...... & 366 \\
\hline - & Avonica.................... & - Bourguignat........ & 304 \\
\hline 一 & nica................. & ain........... & 217 \\
\hline - & ensis............... & 1.............. & 287 \\
\hline- & 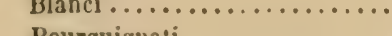 & Bourguignat....... & 233 \\
\hline 一 & Borysthenica. & Keynicki... & 207 \\
\hline & . Dorystiencd. & & 300 \\
\hline
\end{tabular}




\begin{tabular}{|c|c|c|c|}
\hline donta & Briandiana..... & Servain ............ & 299 \\
\hline - & Bronni....................... & D'Ancona ........... & 364 \\
\hline - & Broti ..................... & Bourguignat......... & 210 \\
\hline - & Browni................ & id. $\quad \ldots \ldots \ldots$ & 222 \\
\hline- & Brusinœ................... & Letourneux........... & 353 \\
\hline - & callidœa......$\ldots \ldots \ldots \ldots$ & n........ & 115 \\
\hline- & callosa ..................... & Held.... .......... & 98 \\
\hline - & canescens $\ldots \ldots \ldots \ldots \ldots \ldots$ & Stentz............. & $36 \mathrm{~h}$ \\
\hline - & capitata................... & Kuster.............. & 230 \\
\hline - & cariosa.................... & id. $\quad \ldots \ldots \ldots \ldots \ldots$ & 147 \\
\hline- & carisiana................... & Mabille............. & 273 \\
\hline - & Carotœ.................... & Bourguignat......... & 292 \\
\hline - & Castroi................... & id. $\quad \ldots \ldots \ldots$ & 186 \\
\hline - & castropsis .................. & Fagot................ & 189 \\
\hline - & cellensis, var. cariosa........ . & Mörch .............. & 141. \\
\hline - & id. var. dilatata. ..... & Brot............... & 171 \\
\hline - & var. inflata........ & Rossmassler.......... & 122 \\
\hline - & var. intermedia..... & Brot............... & 228 \\
\hline - & var. 3. minor. ..... & Mörch ............... & 208 \\
\hline - & var. rostrata....... & Brot................ & 147 \\
\hline - & var. sulcata .. .... & Mörch................. & 141 \\
\hline - & Charpentieri ................. & Kuster.............. & 215 \\
\hline - & Charpyi.................... & Dupug............... & 127 \\
\hline - & Clessini $\ldots \ldots \ldots \ldots \ldots$ & Bourguignat.......... & 287 \\
\hline - & $\cos =1.21 \mathrm{a}, \ldots \ldots \ldots \ldots \ldots \ldots$ & Potiez et Michaud..... & 274 \\
\hline - & codiella .................... & Bourguignat.......... & 289 \\
\hline - & collaris ................... & Parreyss............ & 366 \\
\hline - & colloba...........$\ldots \ldots$ & Bourguignat.......... & 302 \\
\hline - & complanata.................. & Ziegler.............. & 26 \\
\hline - & ............... & Rossmässler ........... & 38 \\
\hline - & n............. & Nordenskiold et Nylander. & r. 41 \\
\hline - & .......... & Clessin............. & 236 \\
\hline- & compressa................... & Parreyss............ & 366 \\
\hline - & condatina ................. & Letourneux........... & 147 \\
\hline - & confervigera................. & Schlütter............. & 367 \\
\hline 一 & hodon)... & Brown.............. & 371 \\
\hline- & cordata...$\ldots \ldots \ldots \ldots \ldots$ & Bourguignat......... & 122 \\
\hline - & Cordieri..................... & Ch. d'Orbigny........ & 364 \\
\hline - & ................ & Kutschig............. & 367 \\
\hline - & ........... & Parreyss............. & 367 \\
\hline - & 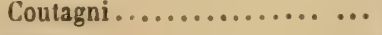 & Bourguignat.......... & 205 \\
\hline- & …....... & Fitzinger............ & 367 \\
\hline - & crimeana.................... & Bourguignat.......... & 377 \\
\hline
\end{tabular}




\begin{tabular}{|c|c|c|}
\hline donta & Kutschig.......... & 8 \\
\hline 一 & cygnœa ................ Rossmässler..... 107, & 108 \\
\hline - & ................. Bourguignat ........ & 140 \\
\hline - & var................ Lecomte.......... & 341 \\
\hline- & var. cordata.......... Rossmässler .......... & 122 \\
\hline- & var. Forchhammeri... Mörch............. & 122 \\
\hline - & var. intermedia....... Joannis............. & 146 \\
\hline - & var. lirata........... Mörch.............. & 128 \\
\hline - & var. Rossmässleri..... Malzine............. & 370 \\
\hline - & cymbalica................. Drouët............. & 372 \\
\hline - & Cyrea..................... id. $\ldots \ldots \ldots$ & 375 \\
\hline - & cyrtoptychia ............... Bourguignat......... & 136 \\
\hline - & cuneata ................... Kuster : .......... & 67 \\
\hline - & dalmatina.................. Kutschig............ & 232 \\
\hline - & 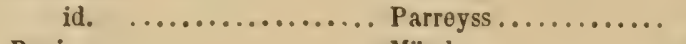 & 367 \\
\hline - & Danica.................... Mörch.............. & 252 \\
\hline - & Dantessantyi................ J. Ray............. & 363 \\
\hline - & Debettana.................. Martinati............ & 193 \\
\hline - & dentata (mytilus)......... Turton.............. & 367 \\
\hline - & dentiens............... & 267 \\
\hline - & Ziegler.............. & 367 \\
\hline - & depressa................... Schmidt............ & 221 \\
\hline - & 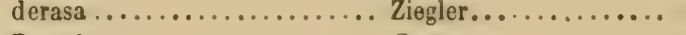 & 367 \\
\hline - & Desori $\ldots \ldots \ldots \ldots \ldots \ldots$ Coutagne $\ldots \ldots \ldots \ldots$. & $30 \stackrel{3}{ }$ \\
\hline- & diminuta................. Bourguignat......... & 229 \\
\hline- & Doëi....................... id. & 169 \\
\hline- & dorsuosa .................. Drouët............ & 372 \\
\hline- & Dubreili... .............. Servain ............. & 329 \\
\hline - & Dupuyi................... Ray et Drouët......... & 202 \\
\hline - & ectina.................... Bourguignat......... & 178 \\
\hline - & elachista.................. & 363 \\
\hline - & ellipsopsis................. & 150 \\
\hline - & èlongata................... Joba............... & 46 \\
\hline - & 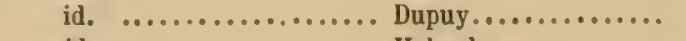 & 48 \\
\hline - & 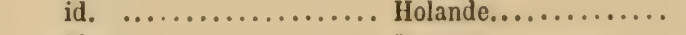 & 48 \\
\hline - & id. $\ldots \ldots \ldots \ldots \ldots \ldots \ldots$. Stentz...$\ldots \ldots \ldots \ldots$ & 227 \\
\hline - & 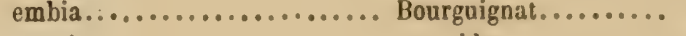 & 180 \\
\hline - & emeria........................... & 283 \\
\hline - & episema................. & 360 \\
\hline - & ervica.. .................. & 271 \\
\hline - & eucypha................. & 103 \\
\hline - & eumorphia................ Letourneux. & 109 \\
\hline - & ourguignat.......... & 294 \\
\hline
\end{tabular}




\begin{tabular}{|c|c|c|}
\hline onta. & .. Ziegler.............. 36 & 367 \\
\hline - & .......... Bourguignat ........ 35 & 356 \\
\hline - & exulcerata................. Villa............. 2? & 275 \\
\hline - & id. $\quad \ldots \ldots \ldots \ldots \ldots \ldots$ Clessin............... 28 & 287 \\
\hline - & Fagoti....................... Bourguignat...... 14 & 144 \\
\hline - & 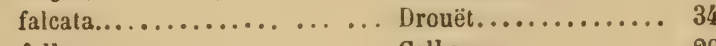 & 347 \\
\hline - & fallax.............................. Colbeau..... 29 & 297 \\
\hline - & fluviatilis................. Dillwyn.......... 36 & 368 \\
\hline- & Forchhammeri............ Bourguignat ........ & 122 \\
\hline- & fragilis $\ldots \ldots \ldots \ldots \ldots \ldots \ldots$ Fitzinger......... ${ }^{20}$ & $? 68$ \\
\hline- & fragilissima.............. Clessin ........... & 129 \\
\hline- & fragillima................ Bourguignat........ & 129 \\
\hline- & . Férussac............ 3 & 368 \\
\hline- & fuscata .............. Ziegler.......... & 368 \\
\hline - & Gallandi................ Bourguignat ...... 95, 1 & 173 \\
\hline - & gallica...$\ldots \ldots \ldots \ldots \ldots \ldots$ id. $\ldots \ldots \ldots$ & 123 \\
\hline - & Gardanensis ..................... Matheron & 364 \\
\hline - & gastroda...................... Bourguignat..... & 136 \\
\hline- & eorgiana................. Drouët........... & 375 \\
\hline- & gibba............................... & 136 \\
\hline- & id. ................ Kutschig....... 276,3 & 368 \\
\hline - & glabra................. Ziegler ........ 221, 3 & 368 \\
\hline - & 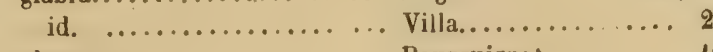 & 262 \\
\hline- & glyca ................. Bourguignat....... & 167 \\
\hline - & Gougetana.............. Ogérien......... 2 & 202 \\
\hline- & Gratelu, eana ............ Gassies. ........... & 29 \\
\hline - & gravida ................ Drouët........... 1 & $109^{\circ}$ \\
\hline- & grossa................... Parreyss .......... 3 & 368 \\
\hline - & liueretini................ Servain .......... 2 & 203 \\
\hline - & Hazayana................... id. 3 & 322 \\
\hline - & Heeri................ (. Mayer......... 3 & 365 \\
\hline - & helvetica................. Bourguignat... 2 & 234 \\
\hline- & id. $\ldots \ldots \ldots \ldots \ldots \ldots$..... Mörch $\ldots \ldots \ldots \ldots$ & 235 \\
\hline- & Humberti............. Bourguignat....... 2 & 213 \\
\hline - & Hydatina..................... Servain 2 & 219 \\
\hline- & idrina.................. Spinelli $\ldots \ldots \ldots \ldots, 2$ & 264 \\
\hline- & id. var. Tra-ymenica...... Rossmässler (suites à).. 3 & 307 \\
\hline - & illota.................. J. Hay ........... 2 & 20 \\
\hline - & illuviosa ..................... Bourguignat..... & $29 \ddot{0}$ \\
\hline - & ilysœca ................. & 341 \\
\hline 一 & incrassata................ & 30 \\
\hline- & ster............. 2 & 208 \\
\hline & & \\
\hline
\end{tabular}


Anodonta intermed $12 \ldots . \ldots \ldots \ldots \ldots \ldots$. . . Pfeiffer......... $2: 3$

id. $\ldots \ldots \ldots \ldots \ldots$.................. 311

id. var. B........ id. ........ $3 z 3$

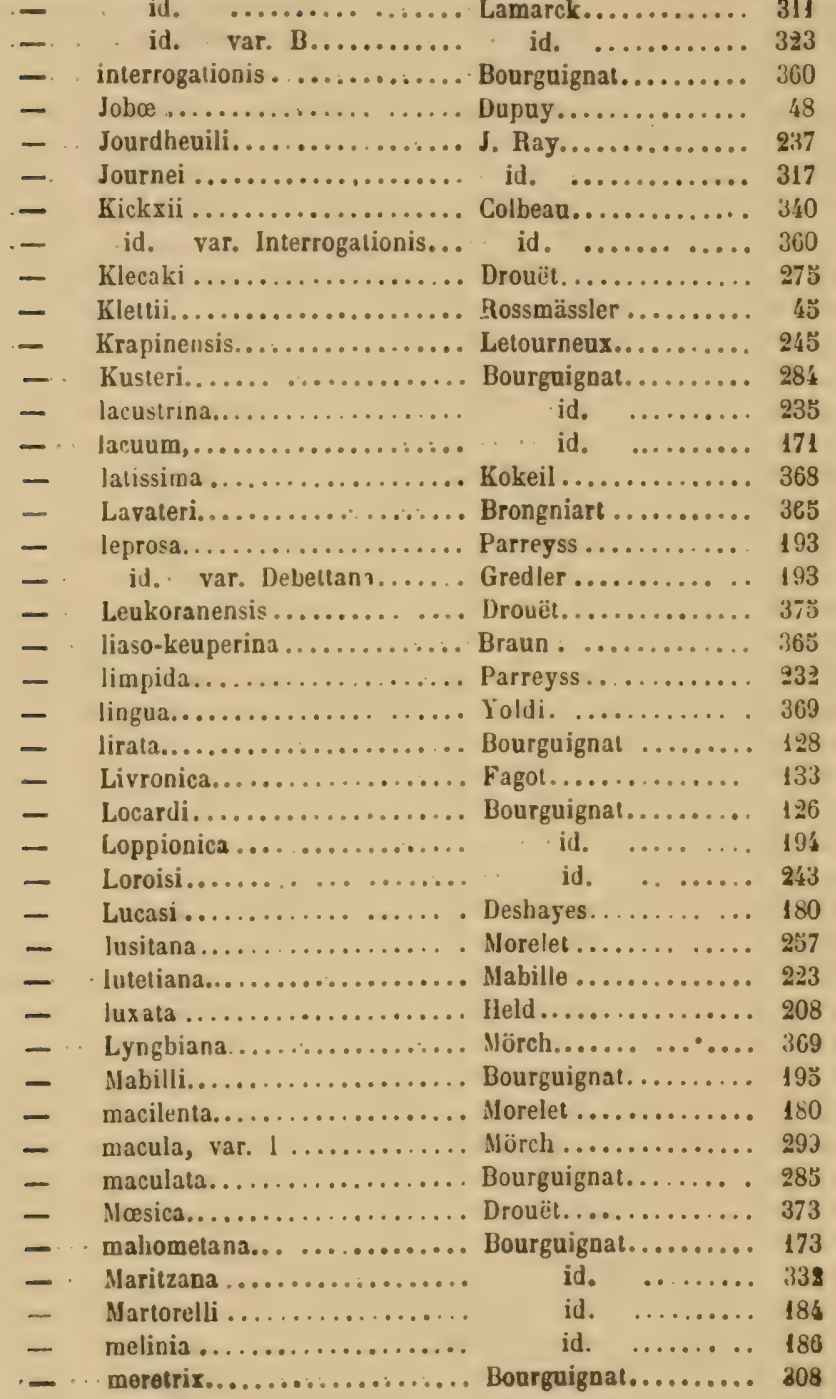




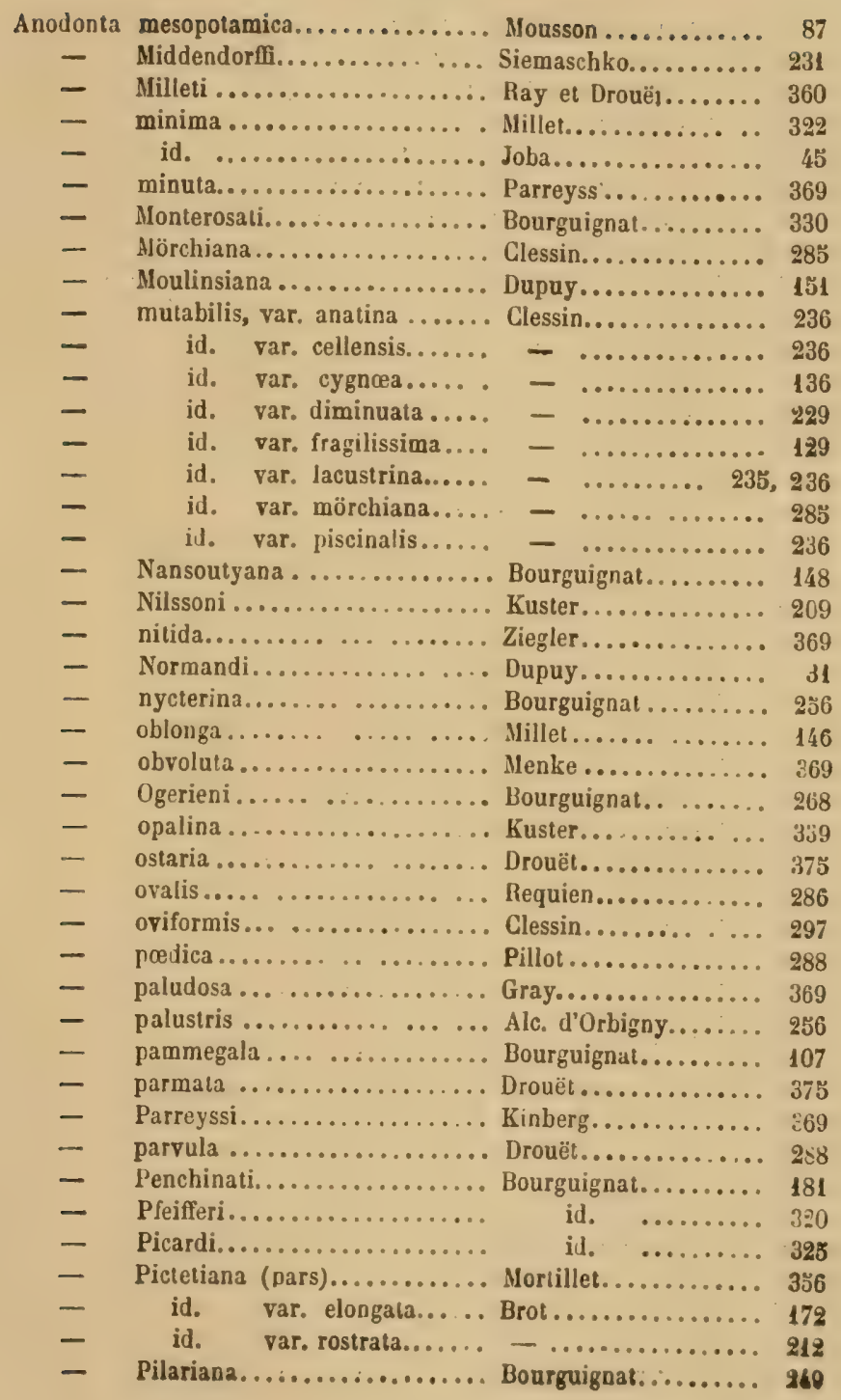




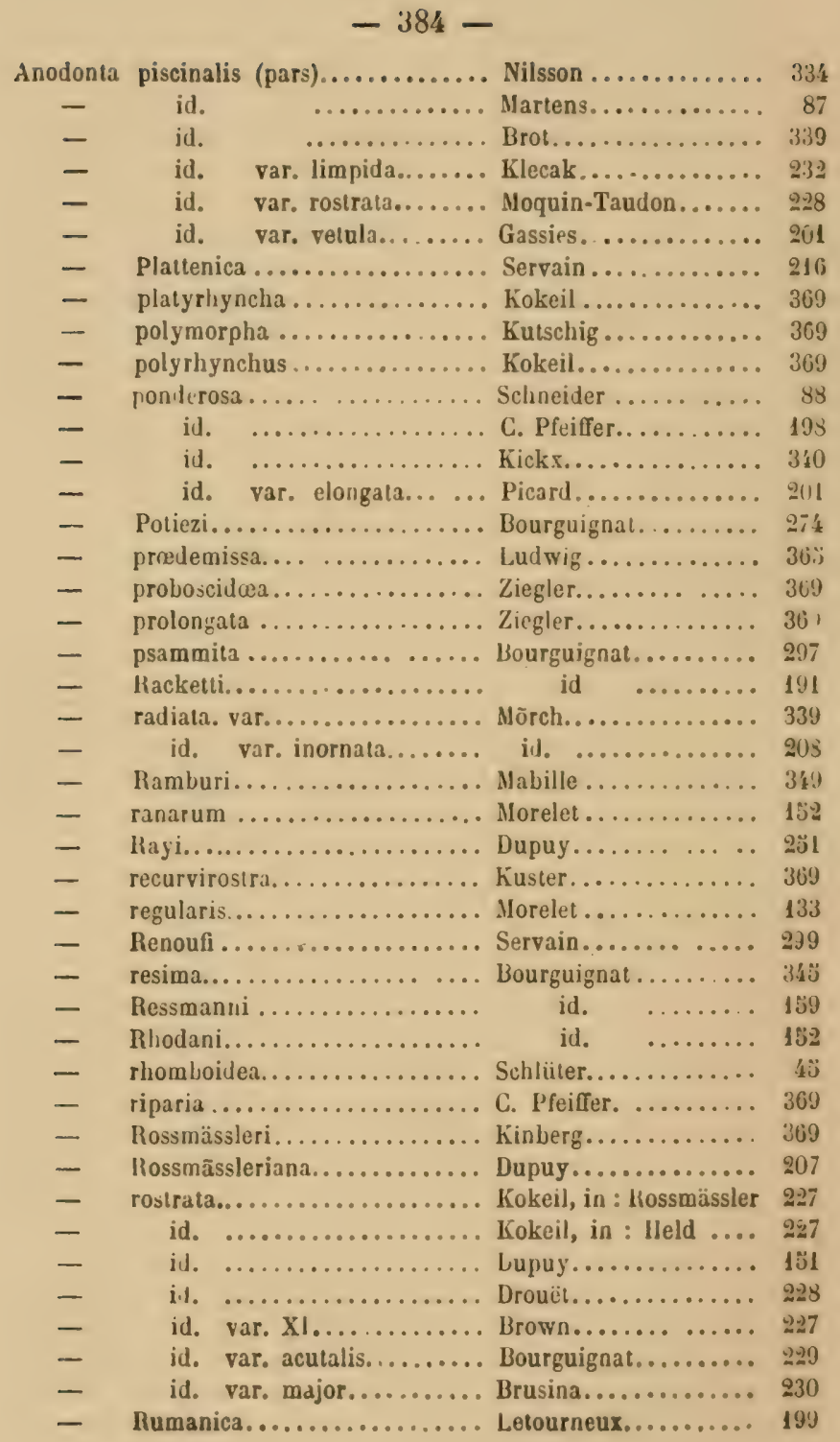




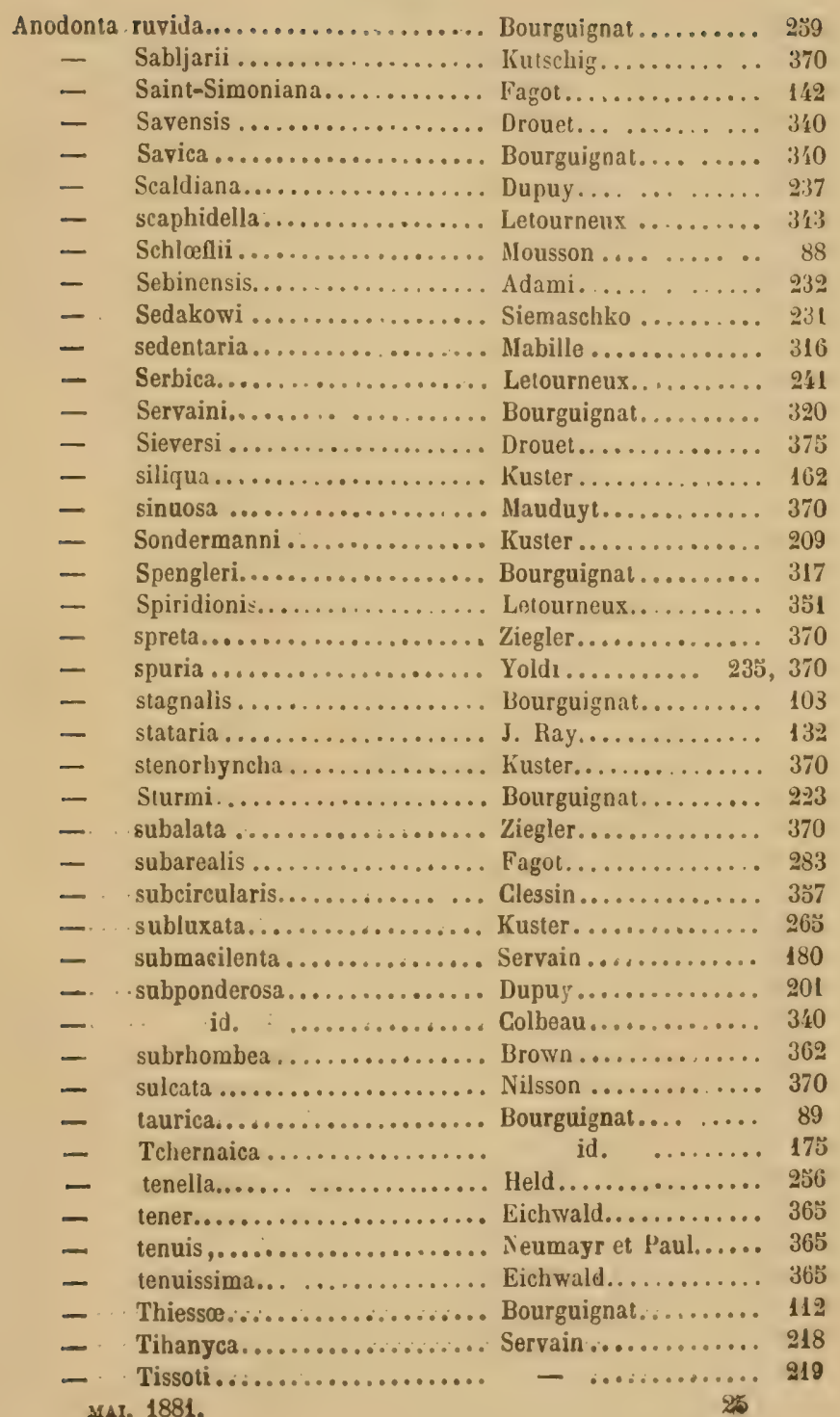


Anodonta trapesialis............... Lamarck........... 334

- Trasymenica.............. Rossmässler (suites à), ..307

- triangularis............ Vidovic........... 370

- triangulata............... Parreyss.......... -370

- tricassina................ Pillot............ 323

- trilonum................. Coutagne.......... 162

- $\quad$ truncata................. Parreyss ........... 300

- tumida..................Kuster............ 212

- id. ............... Fritzinger...... 212, 371

- ventricosa................ C. Pfeiffer......... 119

- id. (juvenilis) ....... id. ........ 320

- id. .............. Kuster....... 121, 284

- id. .............. Dupuy.......... 121

- id. $\quad \ldots \ldots \ldots \ldots \ldots \ldots$ Joannis............ 121

- id. ................ Spinelli........... 193

- il. var............ Middendorff........ 34?

- Vescoiana............... Bourguignat........ 87

- Vescoiana, var. turgidula..... Mlousson........... 87

- vetula ............... Ziegler........... 371

- virens ............... Stentz .......... 371

- viriala .............. Servain ......... 18ü

- viridis ............... Luilwig.......... $36 \mathrm{~s}$

- Westerlundi.............. Fagot............. 266

Colletopterum eximium ............ Bourguignat........ 80

- Lelourneuxi ........... id. ....... 76

- proclarum ............ id. ....... 78

- Tanousi............... id. ....... 83

Musculus maximus planior........... Schröter.......... 107

Mya truncata..................... Schröter........... 139

Mytilus anatinus ................ Sheppard....... 123, 254

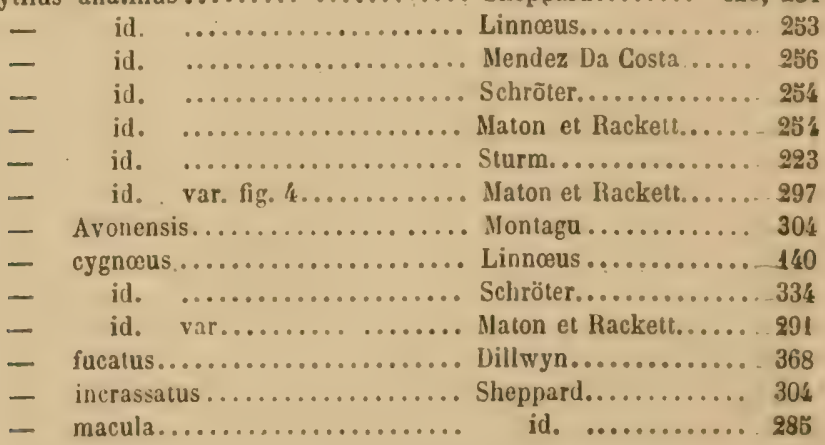


Mytilus stagnalis

Sowerby 108

Pseudanodonta Berlani.

Bourguignat ........ 36

- complanata............

id. $\ldots \ldots \ldots .26$

- Danubialis.............

id. $\ldots \ldots \ldots .21$

- ellipsiformis............

- elongata..............

id. $\ldots \ldots \ldots . \quad 39$

- globosa ..............

id. $\ldots \ldots \ldots, 48$

id. $\quad \cdots \cdots \cdots+31$

Grateloupiana.......... id. ........ 29

- Klettii.............. id. ....... 45

- Letourneuxi.......... id. ........ 19

- Ligeriea.............. Servain ........... 50

- mecyna............ Letourneux......... 24

- Nordenskioldi ......... Bourguignat........ 41

- Normandi............ id. ........ 31

- Pancici .............. Letourneux.......... 32

- Penchinati............ Bourguignat........ 23

- $\quad$ proclara ............. Letourneux........... 16

- Rayi............... Mabille........... 43

- Rossmässleri........... Bourguignat..... 38, 236

- $\quad$ scrupœea ............ id. ........ 34

- Tanousi........ ... Lelourneux......... 42

FIN DELA TABLE

4457 - Implimerie de Poissy - S. Lwsar et Go. 




364. W.

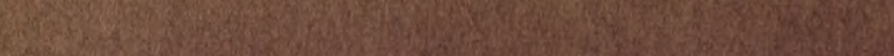

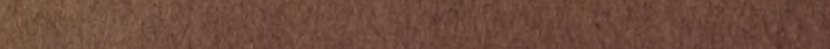

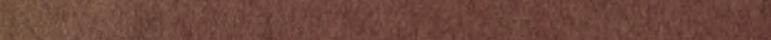

W.

(5)

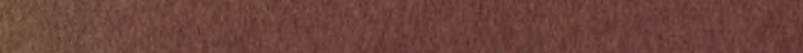

(1)

What

W.

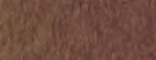

(6)

4.

axive

67

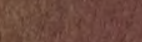



SMITHSONIAN INSTITUTION LIBRARIES ||||||||||||||||||||||||||||||||||||||||||||||||||||||||||||| 39088005949987 